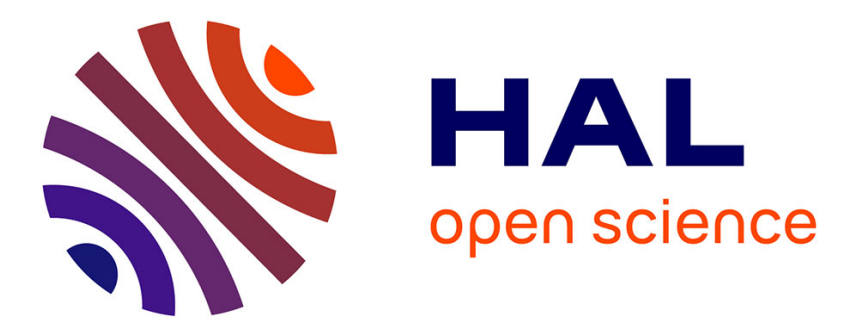

\title{
Relationship between structure and entropy contributions in an anthraquinone mercapto derivative
}

\author{
Maciej Roman, Agnieszka Kaczor, Malgorzata Baranska
}

\section{To cite this version:}

Maciej Roman, Agnieszka Kaczor, Malgorzata Baranska. Relationship between structure and entropy contributions in an anthraquinone mercapto derivative. Journal of Molecular Modeling, 2010, 16 (10), pp.1549-1557. 10.1007/s00894-010-0654-3 . hal-00569393

\section{HAL Id: hal-00569393 https://hal.science/hal-00569393}

Submitted on 25 Feb 2011

HAL is a multi-disciplinary open access archive for the deposit and dissemination of scientific research documents, whether they are published or not. The documents may come from teaching and research institutions in France or abroad, or from public or private research centers.
L'archive ouverte pluridisciplinaire HAL, est destinée au dépôt et à la diffusion de documents scientifiques de niveau recherche, publiés ou non, émanant des établissements d'enseignement et de recherche français ou étrangers, des laboratoires publics ou privés. 


\section{Editorial Manager(tm) for Journal of Molecular Modeling}

Manuscript Draft

Manuscript Number: JMM01081R1

Title: Relation between the structure and entropy contributions in anthraquinone mercapto derivative Article Type: Original paper

Keywords: anthraquinone, ab initio, DFT, entropy effect, conformational analysis

Corresponding Author: Dr. Malgorzata Baranska,

Corresponding Author's Institution: Jagiellonian University

First Author: Maciej Roman, Msc

Order of Authors: Maciej Roman, Msc; Agnieszka Kaczor, Dr.; Malgorzata Baranska

Abstract: The structural and thermodynamic properties of an anthraquinone derivative were studied by means of quantum-chemical calculations. As a result of the conformational analysis fourteen lowenergetic conformers were found by using ab initio and DFT methods. In order to discuss similarities and differences in entropy of the conformers, the rotational and vibrational contributions to entropy were correlated with changes in the conformer structure. The moment of inertia perpendicular to the molecular plane gives significant input to the $\Delta$ Srot, whereas the largest contributions to the $\Delta$ Svib have the vibrations associated with the $\mathrm{\tau S} 1 \mathrm{C} 20$ coordinate.

Response to Reviewers: Dear Prof. Andrzej Sokalski

Editor in Chief of Journal of Molecular Modeling,

I would like to thank the Referees for a careful reading of our manuscript and giving us the valuable comments that with no doubts will improve this paper (Mns.\# JMM01081). Please find below my answers to the Referees' suggestions and comments.

\section{Reviewer \#1:}

Major Revisions

1. Some anthraquinones are proved to have anti-cancer and antifungal properties, as stated by the authors in the introduction. There, the anthraquinones are not in gas phase, but solved in the liquid phase or are interacting with the target in the liquid phase. In contrast, the calculations of the authors are performed in the gas phase. Thus, the authors should discuss, if their results have significance in drug research and for interaction between ligand and target.

The molecular structure defined in the gas phase is a commonly accepted starting point in studies concerning biological activities of molecules. Indeed, we agree with the referee that the future research dealing strictly with biological activity of anthraquinones would require introduction of the solvent and simulation of a biological environment in computational models that is, by the way, a nontrivial problem. However, our studies on antraquinones brought us to finding of the entropic effect in the structures of the MEAA conformers and we decided to analyze this effect in detail. Therefore, our work, although mentioning biological functions of MEAA conformers, was in fact aimed to study the correlation of the rotational and vibrational contributions to entropy with changes in the conformer structure and to do this, the simplest possible model (the gas phase) occurred to us as the most 
preferable. In order to clarify our point of view we suggest adding two more sentences to Introduction (after : The highest emphasis was made on detailed analysis of the entropy effect in order to understand the relation between the structure and entropy contributions (translational, electronic, rotational and vibrational). The computations were done in the gas phase to simplify the computations. Nevertheless, it is hoped that this study can be a starting point in the future research concerning modeling of the biological activity of the MEAA in more complicated systems, mimicking the biological environment.

2. How was the analysis of the potential energy performed? How was guaranteed that all local minima were detected? These two points need a more detailed description.

The analysis of the potential energy surface was performed by investigation of the relationship between energy and dihedral angles. There are four single bonds in the MEAA molecule, which determine the spatial configuration of MEAA. The side-chain of MEAA molecule can loosely rotate around these bonds. For the first bond, defined by C18 and N3 atoms, and dihedral angle defined as? (H23N3-C18-C16) the investigation of the relationship between energy and dihedral angle was performed by optimization of 12 structures, which defer by the value of above-mentioned dihedral angle (this angle was changed by $30^{\circ}$ ), and showed that there are two minima on the PES, at ca. $0^{\circ}$ and ca. $180^{\circ}$. The minimum at ca. $180^{\circ}$ has higher energy than the other one by ca. $48 \mathrm{~kJ} \mathrm{mol-1}$, so it was neglected during further considerations because of extremely low stability (large $\Delta G$ value) of a corresponding conformer. For the next bonds the performance was similar, hence we found 14 lowenergy local minima, which are not all local minima for the MEAA compound. However, conformers obtained from remaining local minima would have very low $\Delta G$ values and would be incredibly unstable.

3. The authors should discuss, if their calculated results can be compared with experimental data. According to our best knowledge the experimental data of MEAA are not available, so we were not able neither to present nor discussed them.

4. Were calculations performed for derivatives with $\mathrm{OH}, \mathrm{NH} 2$ or $\mathrm{CH} 3$ instead of $\mathrm{SH}$ ? This could give perhaps information about the importance of the sulfur.

Derivatives of other type were not investigated, but indeed such calculations could bring more details about the importance of the sulfur. Especially the substitution of MEAA with OH group could result in stronger intermolecular hydrogen bond and consequently would influence torsion vibrations and Svib. It could be a subject of our future study.

5. In Figure 3 and Table 2, values with regard to $G, H$, deltaH, ... are given. How were these values calculated? The corresponding formulas should be presented in more detail.

The calculations of thermochemical values that we discussed in our work are based on standard thermochemical equations that can be found in any standard text on thermodynamics. In fact, we did cite the classic book by McQuarrie and Simon (reference 25) that shows in detail the way of computing these values. We assume that an average reader of the Journal of Molecular Modeling can anytime get familiar with this common knowledge based on the given reference and we would rather like to avoid further complicating our text by adding these equations that can be found elsewhere.

Minor Revisions

6. Please define all abbreviations at their first use, e.g. page 3, TD-DFT

Abbreviation of TD-DFT was defined as suggested. However, concerning other abbreviations such as SVWN, B3LYP, B3PW91 etc., we assume that an average reader of the Journal of Molecular Modeling is familiar with this common nomenclature.

7. For no missunderstanding, the indices in $\mathrm{N} 3 \mathrm{H} 23$ and 05 should be given as upper indices (page 5). In the present form, it looks like chemical formulas, which can lead to misunderstandings 
The indices describing atoms were changed into normal numbers, e.g. instead of N3H23 we put N3H23, according to the nomenclature commonly used in papers published in Journal of Molecular Modeling.

Reviewer \#2:

In this work authors present their results on the conformational properties and related thermodynamics of an antraquinone molecule. They conclude several important statements with probable wide interest justifying publication in the Journal of Molecular Modeling. The work is interesting, well organized, referencing is up to date and comprehensive. This work clearly shows how the mechanical property (moment of inertia) can participate in determination of these very important chemical properties. By discussing that information this work highlights the entropy - reactivity relation in general and because of that I suggest to publish this work as it is or subject to the following minor remarks:

The titles of the axe of figure in the graphical abstract are not visible.

The graphical abstract was improved in order to see the titles of the axes of figure.

Ref. 16: Gaussian not allows restricted citation by listing only the first author.

The citation was corrected according the Reviewer suggestion.

The number of tables about anyway very useful data (normalmodes, etc.) is enormously large.

Therefore I suggest to publish those information as supplemental file attached to the online publication. Only two tables are included in the text, the remaining are gathered in Supplementary Materials.

With regards,

Malgorzata Baranska 
Abstract graphic
Click here to download Abstract graphic: graphical abstract-graph-rev.doc

Abstract graphic
Click here to download Abstract graphic: graphical abstract-graph-rev.doc

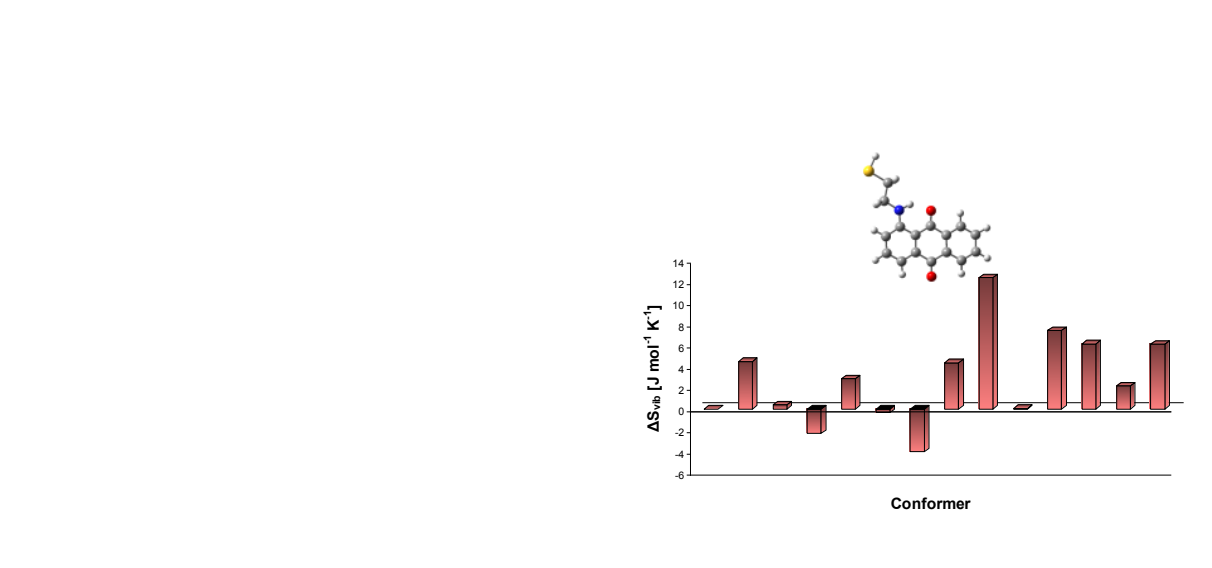

Conformer 


\title{
Relation between the structure and entropy contributions in anthraquinone mercapto derivative
}

Received: 02.11.2009 / Accepted: 13.01.2010

Maciej Roman, Agnieszka Kaczor, and Malgorzata Baranska ${ }^{\bowtie}$

Faculty of Chemistry, Jagiellonian University, 3 Ingardena Str., 30-060 Kraków, Poland

$凶$ Tel.: +48 12 6632253; Fax. +48 12 6340515; Email: baranska@chemia.uj.edu.pl

\begin{abstract}
The structural and thermodynamic properties of an anthraquinone derivative were studied by means of quantum-chemical calculations. As a result of the conformational analysis fourteen low-energy conformers were found by using $a b$ initio and DFT methods. In order to discuss similarities and differences in entropy of the conformers, the rotational and vibrational contributions to entropy were correlated with changes in the conformer structure. The component of the moment of inertia perpendicular to the molecular plane gives significant input to $\Delta S_{\text {rot }}$, whereas the largest contributions to the $\Delta S_{\text {vib }}$ have the vibrations associated with the $\tau_{\mathrm{S} 1 \mathrm{C} 20}$ coordinate.
\end{abstract}

Keywords Anthraquinone $\cdot$ Ab initio $\cdot$ DFT $\cdot$ Entropy effect $\cdot$ Conformational analysis 


\section{Introduction}

Anthraquinones have recently attracted increasing attention due to their various biological activities and a wide range of applications in the pharmaceutical industry [1-4]. Additionally, some anthraquinones have been proved to posses anti-cancer and antifungal properties [2, 3]. They are also known as a family of photosensitizers, which photo-generate reactive oxygen species, such as ${ }^{1} \mathrm{O}_{2}$ and $\mathrm{O}_{2}^{-}[5-8]$. Thus, anthraquinones have been studied by using quantumchemical methods, mainly TD-DFT (Time-Dependant Density Functional Theory), in order to discuss their electronic spectra [9-11].

The hydrophobic effect, side-chain conformational entropy, steric factors, and main-chain electrostatic interactions have been indicated as the dominant physical factors that determine conformational preferences of amino acids in proteins [12]. In particular, it has been demonstrated that restriction of amino acids side-chain motion is enthalpically favorable, but unfavorable in terms of Gibbs energy and entropy [13]. The stabilization of some conformations on the Gibbs free energy and entropy ground, manifesting as increase of their abundance, was previously described for phenylalanine and tryptophan and called "the entropy effect" [14, 15]. Structurally, this effect was related to the strength of the intramolecular H-bond and steric hindrance in amino acids conformers [14, 15].

In our studies on anthraquinone derivatives, we found out that 1-[(2-mercaptoethyl)amino]9,10-anthraquinone (MEAA) (Fig. 1) shows a significant entropy effect. Therefore, we undertook an analysis of structural and thermodynamic properties of MEAA by using quantum-chemical methods in order to shed some light on reasons for entropic stabilization/destabilization of conformers. The highest emphasis was made on detailed analysis of the entropy effect in order to understand the relation between the structure and entropy contributions (translational, electronic, rotational and vibrational). The computations were done in the gas phase to simplify the computations. Nevertheless, it is hoped that this study can be a starting point in the future research concerning modeling of the biological activity of the MEAA in more complicated systems, mimicking the biological environment. 


\section{Calculations}

MEAA geometries and harmonic vibrational frequencies were calculated using the Gaussian 03 package of programs [16]. The conformational analysis was carried out at the B3LYP/6$31 \mathrm{G}$ level of theory in order to find all low-energy conformers of the investigated compound. The obtained geometries of MEAA conformers were optimized by using two methods: Hartree-Fock (HF) and density functional theory (DFT), combined with the 6-31G(d,p) basis set. In case of DFT method four functionals were applied: local SVWN [17, 18], gradient BLYP [19, 20], hybrid B3LYP [20, 21] and B3PW91 [21, 22]. Additionally, the optimization of the low-energy conformers was carried out at the B3LYP/6-311++G(d,p) level.

Vibrational frequencies were calculated for the low-energy conformers at each level of theory and the nature of minima on the potential energy surface (PES) resulting from optimization were determined by inspection of the corresponding calculated Hessian matrix in order to verify that true minimum energy conformations were considered. The relative abundances of the fourteen most stable conformers were then estimated at the room temperature using the $\Delta \mathrm{G}=\mathrm{RT} \ln \mathrm{K}_{\mathrm{c}}$ equation, where $\Delta \mathrm{G}$ is the Gibbs free energy relative to the most stable conformer and $\mathrm{K}_{\mathrm{c}}$ is the ratio of the concentrations of a pair of conformers.

Potential energy distributions (PED) of the normal modes were computed in terms of natural internal coordinates [23] with the GAR2PED program [24] in order to discuss vibrational entropy contribution to the entropy effect.

\section{Results and discussion}

\section{Conformational analysis}

Fourteen low-energy conformers of MEAA were obtained as a product of the conformational analysis performed at the B3LYP/6-311++G(d,p) level (Fig. 2). The selected dihedrals defining the conformers are listed in Table 1.

Since the occurrence of conformers results from the different arrangement of the side-chain of MEAA, their spatial configuration is related to three dihedral angles defined as: $\angle$ (C20-C2$\mathrm{N} 3-\mathrm{C} 18), \angle(\mathrm{S} 1-\mathrm{C} 20-\mathrm{C} 2-\mathrm{N} 3)$ and $\angle(\mathrm{H} 33-\mathrm{S} 1-\mathrm{C} 20-\mathrm{C} 2)$. The conformer notation applied in this paper is based on the values of proper dihedral angles and consists of three elements. The first 
one corresponds to the value of the C20-C2-N3-C18 dihedral angle: $c a .80^{\circ}$ (synclinal, $S c$ ), ca. $110^{\circ}$ (anticlinal, Ac), or ca. $180^{\circ}$ (trans, T). The second and third one is related with the conformation of the S1-C20-C2-N3 and the H33-S1-C20-C2 dihedral angle, respectively (trans, $T$, gauche, $G$, and minus gauche, $-G$ ).

In most cases differences between MEAA conformer geometries calculated by using ab inito and DFT methods with the same basis set are not significant. The largest discrepancy was found for the structures obtained from the HF and SVWN calculations.

The intramolecular hydrogen bond between the $\mathrm{N} 3 \mathrm{H} 23$ group and the $\mathrm{O} 5$ oxygen atom is one of the characteristic features of all low-energy MEAA conformers (see Supplementary Materials). For all considered structures, the B3LYP/6-311++G(d,p) calculations predict the N3----O5 distance in the 2.624-2.637 $\AA$ range and the $\angle(\mathrm{N} 3-\mathrm{H} 23----\mathrm{O} 5)$ angle in the range of 133.5-138. $1^{\circ}$. For most MEAA conformations, the distance between the H23 and O5 atoms vary in the 1.801-1.810 $\AA$ range (B3LYP/6-311++G(d,p)) and is predicted to be slightly shorter only for $A c-G T, A c-G G$ and $A c-G-G$ conformers ( $c a .1 .78 \AA$ ) and somewhat longer for $T G T$ and $T G G$ forms ( $c a .1 .82$ and $1.83 \AA$, respectively). The obtained distances and angles indicate that the H-bond predicted for all considered conformations is of comparable strength. The length of the H-bond calculated by using the B3LYP and BLYP functionals combined with the $6-31 \mathrm{G}(\mathrm{d}, \mathrm{p})$ basis set is very similar to that obtained from calculations with the larger basis set. Shorter H-bonds were obtained from the B3PW91 and SVWN calculations, while the H-bonds obtained from HF calculations are longer. Nevertheless, a qualitative agreement between all applied methods is satisfactory.

\section{Thermochemistry of MEAA conformers}

There are some principal physical factors that determine the conformational state of a molecule. In general, the role of intramolecular interactions of the side chain is crucial, however, in case of MEAA conformers significant is the side-chain conformational entropy and steric factors. Since the restriction of internal rotations results from the steric effects, it has an important contribution to the conformers stability [25].

The calculated relative zero-point-corrected energies and Gibbs free energies for all stable MEAA conformers are presented in Fig. 3. It can be seen that the relative zero-point-corrected energies do not follow the order of Gibbs free energies. Thus, it is evident that the entropy effect is of importance for the thermochemistry of MEAA conformers and it should be taken 
into account considering the conformers stability. In case of MEAA, the H-bond does not differentiate the entropy of the structures, as the H-bond predicted in all conformers are of the same type and similar strength (Fig. 2). Therefore, the compound is a convenient example to correlate the entropy effect with the geometry of the side chain.

The most important basic thermodynamic values and abundances of all considered MEAA conformers are shown in Table 2. In comparison to $S c T-G$, four MEAA conformers are unfavored by entropy ( $A c-G G, S c G G, S c G-G$ and $T G G$ ) due to the negative entropic contribution. The largest effect is observed for the $A c-G G$ form $\left(\mathrm{T} \Delta \mathrm{S}_{\mathrm{tot}}=-1.4 \mathrm{~kJ} \mathrm{~mol}^{-1}\right)$, and is caused by the presence of the steric constrain due to interactions between the hydrogen atom from the mercapto group and the nitrogen atom. Indeed, the SH group is hovering over the nitrogen atom. Thus, the internal rotation of the mercapto group can be considered as internally hindered in this conformer. Analogous behavior is also predicted for the $S c G-G$ conformer that is also significantly destabilized by entropy $\left(\mathrm{T} \Delta \mathrm{S}_{\mathrm{tot}}=-0.9 \mathrm{~kJ} \mathrm{~mol}^{-1}\right.$, Fig. 3$)$.

In comparison to $S c T-G$, the remaining forms are stabilized by entropy and the largest effect is observed for the conformers with a "straight" side-chain, i.e. with the side-chain dihedral angles close to $180^{\circ}$. Close examination of the structures with the highest $\mathrm{T} \Delta \mathrm{S}_{\text {tot }}$ values (TTT, $T G T, S c G T$ and $A c-G T)$ indicates that the key factor is the trans arrangement of the H33-S1C20-C2 group.

The entropy effect has a strong impact on some relative Gibbs free energies and abundances of the considered conformers (Table 2). TT-G has the lowest $\Delta \mathrm{G}$ and the largest abundance, despite a small enthalpic destabilization. Strong entropy effects are also responsible for the non-neglectable abundance of $T T T$ (4.3\%). It should be emphasized that only this very conformer has the degeneracy equal to one.

Calculations of thermochemical values and abundances of conformers were also carried out based on the other methods/basis sets applied in this paper. The results are very similar to these obtained from the B3LYP/6-311++G(d,p) calculations. The most significant differences were noted for the SVWN calculations. Despite all observed differences, there are some general conclusions that can be derived from this analysis. The conformers with considerably twisted side-chains are destabilized by entropy because of the presence of the steric constrains. They are responsible for a restriction of the internal rotation and produce growth of $\Delta \mathrm{G}$ in respect to $\Delta \mathrm{E}_{\text {corr }}$. The increase of $\Delta \mathrm{G}$ results from the decrease of $\Delta \mathrm{S}_{\text {tot }}$, but it is reduced by the decrease of $\Delta \mathrm{H}$. For instance, for the $A c-G G$ conformer $\Delta \mathrm{G}$ is equal to $5.1 \mathrm{~kJ}$ $\mathrm{mol}^{-1}$, despite that $\Delta \mathrm{H}$ equals only $3.6 \mathrm{~kJ} \mathrm{~mol}^{-1}$ (less than $\Delta \mathrm{E}_{\text {corr }}$, see Table 2). It results from 
large entropy effects with the $\mathrm{T} \Delta \mathrm{S}_{\text {tot }}$ shifted down to $-1.4 \mathrm{~kJ} \mathrm{~mol}^{-1}$. The most extended conformers (e.g. TTT, TT-G, ScTT, Ac-GT, ScGT and TGT) are stabilized by entropy. Moreover, $X X T$ conformers ( $X$ denotes any conformation of the relevant dihedral angles) have the mercapto group heading outside of MEAA, so this group does not interact with the remaining part of molecule.

The essential point of this work is to find the relationship between entropy value and the structure of molecule. The total entropy is defined as [25]:

$$
S_{\text {tot }}=R\left(\ln (\text { eq })+T\left(\frac{\partial \ln (\mathrm{q})}{\partial T}\right)_{V}\right)
$$

where $\mathbf{R}$ is the gas constant $\left(\mathrm{R}=8.31441 \mathrm{~J} \mathrm{~mol}^{-1} \mathrm{~K}^{-1}\right)$, $\mathbf{e}$ is the Euler constant $(\mathrm{e}=2.71828), \mathbf{q}$ $=\mathrm{q}(\mathrm{T}, \mathrm{V})$ is the total partition function, defined as $\mathrm{q}=\mathrm{q}_{\text {trans }} \mathrm{q}_{\text {elec }} \mathrm{q}_{\text {rot }} \mathrm{q}_{\text {vib }}$ (where subscripts denote translational, electronic, rotational, and vibrational contribution to partition function) and $\mathbf{T}$ is the temperature. The total entropy can be also defined as the sum of the translational, electronic, rotational and vibrational entropy.

The translational entropy depends on several constants as well as temperature, pressure and mass of a molecule. All these factors are constant when different conformations of the same compound are considered at constant temperature and pressure. The same can be stated for the electronic entropy $S_{\text {elec }}$. Therefore, structure of the conformers is affected only by the rotational and vibrational contributions to entropy, analysed below.

\section{Rotational entropy of MEAA conformers}

Generally, for a nonlinear polyatomic molecule the rotational partition function $\mathrm{q}_{\mathrm{rot}}$ is given by equation [25]:

$$
\mathrm{q}_{\mathrm{rot}}=\frac{\pi^{1 / 2}}{\sigma_{\mathrm{r}}}\left(\frac{\mathrm{T}^{3 / 2}}{\left(\Theta_{\mathrm{r}, \mathrm{x}} \Theta_{\mathrm{r}, \mathrm{y}} \Theta_{\mathrm{r}, \mathrm{z}}\right)^{1 / 2}}\right)
$$

where $\boldsymbol{\sigma}_{\mathbf{r}}$ is the symmetry number for rotation and $\boldsymbol{\Theta}_{\mathbf{r}, \mathbf{i}}$ is the function defined as [25]:

$$
\Theta_{\mathrm{r}, \mathrm{i}}=\frac{\mathrm{h}^{2}}{8 \pi^{2} \mathrm{k}_{\mathrm{B}} \mathrm{I}_{\mathrm{i}}}
$$


where $\mathbf{I}_{\mathbf{i}}$ is the moment of inertia. While the rotational entropy $S_{\text {rot }}$ is expressed by [25]:

$$
\mathrm{S}_{\mathrm{rot}}=\mathrm{R}\left(\ln \left(\mathrm{q}_{\mathrm{rot}}\right)+\frac{3}{2}\right)
$$

The analysis of Eqs. 2-4 shows that rotational entropy depends on some constants as well as temperature and moments of inertia. Since the moment of inertia changes with the structure modification, the rotational entropy is different for different conformers. Therefore, $S_{\text {rot }}$ has an influence on the observed entropy effect.

The relative rotational entropy of all considered conformers calculated at the B3LYP/6$311++\mathrm{G}(\mathrm{d}, \mathrm{p})$ level of theory are presented in Fig. 4A. Two conformers (TTT and TT-G) are stabilized by $S_{\text {rot }}$ referred to that of $S c T-G$ form, but this effect is not significant, since the rotational entropy of both is only $c a .0 .5 \mathrm{~J} \mathrm{~mol}^{-1} \mathrm{~K}^{-1}$. It is correlated with spatial structure extended perpendicularly to the direction of the z-component of the moment of inertia of these conformers. Particularly, these two are the only forms with both C20-C2-N3-C18 and S1-C20-C2-N3 dihedral angles in trans arrangement, see Fig. 3). As the ScTX conformers have similar spatial structure (they differ only by the value of the H33-S1-C20-C2 angle) they have similar $\Delta \mathrm{S}_{\text {rot }}$ values. In comparison to $S c T$ - $G$ conformer, the remaining conformers are destabilized by the rotational entropy. Their $\Delta \mathrm{S}_{\text {rot }}$ values are in the range from $-0.2 \mathrm{~J} \mathrm{~mol}^{-1} \mathrm{~K}^{-1}$ for $T(-) G X$ conformers to $-0.9 \mathrm{~J} \mathrm{~mol}^{-1} \mathrm{~K}^{-1}$ for Ac-GT conformer and, for the forms with the C20-C2-N3-C18 angle in the anticlinal or synclinal arrangement, are considerably lower than those with the angle in trans configuration. The obtained results are practically invariant to the method applied. They bring the conclusion that arrangement of the $\mathrm{C} 20-\mathrm{C} 2-\mathrm{N} 3-\mathrm{C} 18$ chain is a key factor responsible for small rotational stabilization/destabilization.

According to Eqs. 2-4 the rotational entropy depends on a product of moments of inertia. The larger the product the larger $\Delta S_{\text {rot }}$ contribution. Table S.2. shows the $I_{x} \cdot I_{y} \cdot I_{z}$ of all considered MEAA conformers calculated by all applied methods and basis sets. The obtained results definitely confirm the above considerations. As expected, the highest values are predicted for $T T X$ conformers, they are a bit lower for ScTX and the lowest for the ScGX or Ac-GX conformers.

\section{Vibrational entropy of MEAA conformers}

In case of the vibrational partition function $\mathrm{q}_{\mathrm{vib}}$ and the vibrational entropy $\mathrm{S}_{\mathrm{vib}}$, the relationship between these values and the molecular structure is more complicated, because 
all vibrations of the molecule must be taken into consideration. The complexity of the $\mathrm{q}_{\mathrm{vib}}$ and the $S_{\text {vib }}$ interpretation increases with the size of the studied molecule. If the first vibrational level is defined as zero, the vibrational partition function is given by [25]:

$$
\mathrm{q}_{\mathrm{vib}}=\prod_{\mathrm{i}=1}^{3 \mathrm{~N}-6} \frac{1}{1-\mathrm{e}^{-\frac{h v_{\mathrm{i}}}{\mathrm{k}_{\mathrm{B}} \mathrm{T}}}}
$$

where $\mathbf{v}_{\mathbf{i}}$ is the frequency of the $\mathrm{i}$-th normal vibration and $\mathbf{N}$ is the number of atoms in the molecule. While the vibrational entropy is expressed by [25]:

$$
S_{\text {vib }}=R \sum_{i=1}^{3 N-6}\left(\frac{\frac{h v_{i}}{k_{B} T}}{e^{\frac{h v_{i}}{k_{B} T}}-1}-\ln \left(1-e^{-\frac{h v_{i}}{k_{B} T}}\right)\right)
$$

According to Eqs. 5-6 the $S_{\text {vib }}$ depends on some constants and the frequencies of all molecular vibrations, whereas frequencies are associated to internal coordinates and force constants [25]. Thus, the $S_{\text {vib }}$ is strongly dependent on molecular structure and conformation.

Fig. 5A presents the relative vibrational entropies of all considered conformers calculated at the B3LYP/6-311++G(d,p) level. It is clear from this figure that in comparison to $S c T-G$ form most conformers are stabilized by the vibrational entropy and only three of them are destabilized ( $A c-G G, S c G-G$ and $T G G$ ). Among the stabilized conformers, the highest $\Delta \mathrm{S}_{\mathrm{vib}}$ is recognized for $T T T$ form $\left(12.4 \mathrm{~J} \mathrm{~mol}^{-1} \mathrm{~K}^{-1}\right)$, while the most destabilized is $A c-G G\left(-4.1 \mathrm{~J} \mathrm{~mol}^{-1}\right.$ $\left.\mathrm{K}^{-1}\right)$.

Among conformers possessing the same arrangement of the C20-C2-N3-C18 and S1-C20-C2$\mathrm{N} 3$ angles $\Delta \mathrm{S}_{\mathrm{vib}}$ generally decreases in order $X X T>X X G>X X-G$ with the exception of $A c$ $G X$. According to their $\Delta \mathrm{S}_{\mathrm{vib}}$ values, $A c-G X$ conformers can be ordered as follows: $A c-G T>$ $A c-G-G>A c-G G$. This observation brings the conclusion that the arrangement of the mercapto group in respect to the reminding part of the side-chain is the main factor deciding about the vibrational contribution to entropy.

Investigation of the Eq. 6 leads towards very important finding. The largest contributions to the total vibrational entropy have low frequency vibrations. For example, a vibration with frequency of $50 \mathrm{~cm}^{-1}$ gives a contribution of $20.2 \mathrm{~J} \mathrm{~mol}^{-1} \mathrm{~K}^{-1}$, while one with frequency of 500 $\mathrm{cm}^{-1}$ provides an input of only $2.8 \mathrm{~J} \mathrm{~mol}^{-1} \mathrm{~K}^{-1}$. Contributions from stretching vibrations (e.g. 
$\mathrm{C}-\mathrm{H}, \mathrm{N}-\mathrm{H}$ or S-H) are lower than $4 \cdot 10^{-4} \mathrm{~J} \mathrm{~mol}^{-1} \mathrm{~K}^{-1}$; but bending, torsion and skeletal vibrations, which are low-frequency modes, must be taken into the consideration.

Results obtained at other levels of theory specified in the computational part are in slight disagreement with the one obtained using B3LYP/6-311++G(d,p) level of theory. As expected, most dissimilarities are produced by the SVWN calculations. Therefore, a further investigation was carried out only with the results calculated with the B3LYP/6-311++G(d,p) basis set. PEDs of all investigated MEAA conformers and definition of internal coordinates are provided in Tables S.3 to S.17.

In comparison to $S c T-G$ conformer, $\Delta \mathrm{S}_{\mathrm{vib}}$ of $S c T X$ conformers are: $4.4 \mathrm{~J} \mathrm{~mol}^{-1} \mathrm{~K}^{-1}$ for $S c T T$, $0.4 \mathrm{~J} \mathrm{~mol}^{-1} \mathrm{~K}^{-1}$ for $S c T G$, so the $\mathrm{S}_{\mathrm{vib}}$ decreases in order of: $S c T T>S c T G>S c T-G$. Nineteen lowest-frequency vibrations of these conformers have similar values and to some extent are responsible for the same structural changes (similar PEDs). Therefore these vibrations do not differentiate significantly the vibrational entropy of conformers. The opposite situation exists for vibrations associated with $\tau_{\mathrm{S} 1 \mathrm{C} 20}$ - an internal coordinate involving the hydrogen atom $\mathrm{H} 33$, predicted to absorb at $151 \mathrm{~cm}^{-1}, 220 \mathrm{~cm}^{-1}$ and $209 \mathrm{~cm}^{-1}$ for ScTT, ScTG and ScT-G, respectively (Fig. 5B and alternatively Fig. S.18), which considerably differs for abovementioned forms.

Similar considerations can be carried out for $A c-G X$ conformers. $\Delta \mathrm{S}_{\mathrm{vib}}$ of these conformers are: $6.1 \mathrm{~J} \mathrm{~mol}^{-1} \mathrm{~K}^{-1}(A c-G T),-4.1 \mathrm{~J} \mathrm{~mol}^{-1} \mathrm{~K}^{-1}(A c-G G)$ and $2.2 \mathrm{~J} \mathrm{~mol}^{-1} \mathrm{~K}^{-1}(A c-G-G)$ in respect to $S c T-G$. For $A c-G X$ conformers, the order in which the $\mathrm{S}_{\mathrm{vib}}$ decreases as follows: $A c-G T>$ $A c-G-G>A c-G G$. The vibrations of $144 \mathrm{~cm}^{-1}(A c-G T), 276 \mathrm{~cm}^{-1}(A c-G G)$ and $205 \mathrm{~cm}^{-1}$ (Ac$G-G)$ show the contribution of the internal coordinate $\tau_{\mathrm{S} 1 \mathrm{C} 20}$ and have enormous influence on $\Delta \mathrm{S}_{\text {vib }}$ values (Tables S.7, S.8, S.9).

In case of $S c G X$ conformers $\Delta S_{\text {vib }}$ (referred to $S c T-G$ form) is $6.2,0.1$ and $-2.3 \mathrm{~J} \mathrm{~mol}^{-1} \mathrm{~K}^{-1}$ for $S c G T, S c G G$ and $S c G-G$, respectively. For this group the following frequencies are related to internal coordinate $\tau_{\mathrm{S} 1 \mathrm{C} 20}: 135 \mathrm{~cm}^{-1}(S c G T), 192 \mathrm{~cm}^{-1}(S c G G)$ and $238 \mathrm{~cm}^{-1}(S c G-G)$. These vibrational modes and the fact that there are significant differences in frequencies of most remaining vibrations involving the mercapto group (described by internal coordinates defined as: $\delta_{1 / \mathrm{C} 20 \mathrm{H} 31 \mathrm{H} 32}, \delta_{2 / \mathrm{C} 20 \mathrm{H} 31 \mathrm{H} 32}, \tau_{\mathrm{C} 2 \mathrm{C} 20}$ and $\left.\tau_{\mathrm{C} 2 \mathrm{~N} 3}\right)$, allow explaining the values of $\Delta \mathrm{S}_{\mathrm{vib}}$.

TTX conformers have also different $\Delta \mathrm{S}_{\mathrm{vib}}$. For $T T T$ the vibration at $96 \mathrm{~cm}^{-1}$ with $31 \%$ of $\tau_{\mathrm{S} 1 \mathrm{C} 20}$ and for $T T-G$ at $215 \mathrm{~cm}^{-1}$ with $78 \%$ of this coordinate permit to explain the significant difference between $\Delta \mathrm{S}_{\mathrm{vib}}$ values and the stability of TTT. Additionally, other vibrations of TTT involving the $\mathrm{S}-\mathrm{H}$ group have lower values than the corresponding frequencies for $T T-G$. 
For conformers named as $T(-) G X$, the following values of $\Delta \mathrm{S}_{\text {vib }}$, referred to $S c T-G$, are predicted by calculations: $7.4(T G T), 2.9(T-G G)$ and $-0.4 \mathrm{~J} \mathrm{~mol}^{-1} \mathrm{~K}^{-1}(T G G)$. Contributions to the vibrational entropy from vibrations containing $\tau_{\mathrm{S} 1 \mathrm{C} 20}$ coordinate are different, but sufficient to make $T G T$ the most stabilized conformer.

In conclusion, the vibrational modes involving the mercapto group have an important influence on the vibrational entropy changes. It refers especially to vibrations dominated by the $\tau_{\mathrm{S} 1 \mathrm{C} 20}$ internal coordinate. Vibrational frequencies associated with this vibration of conformers strongly stabilized by the vibrational entropy are the lowest: $\Delta \mathrm{S}_{\mathrm{osc}}=12.9 \mathrm{~J} \mathrm{~mol}^{-1}$ $\mathrm{K}^{-1}$ and $v=96 \mathrm{~cm}^{-1}(T T T), \Delta \mathrm{S}_{\mathrm{osc}}=7.4 \mathrm{~J} \mathrm{~mol}^{-1} \mathrm{~K}^{-1}$ and $v=116 \mathrm{~cm}^{-1}(T G T), \Delta \mathrm{S}_{\mathrm{osc}}=6.2 \mathrm{~J} \mathrm{~mol}^{-1}$ $\mathrm{K}^{-1}$ and $v=135 \mathrm{~cm}^{-1}(S c G T)$ and $\Delta \mathrm{S}_{\mathrm{osc}}=6.2 \mathrm{~J} \mathrm{~mol}^{-1} \mathrm{~K}^{-1}$ and $v=144 \mathrm{~cm}^{-1}$ (Ac-GT). Contrarily, these frequencies of conformers strongly entropically destabilized are the highest: $\Delta \mathrm{S}_{\mathrm{osc}}=-4.1$ $\mathrm{J} \mathrm{mol}^{-1} \mathrm{~K}^{-1}$ and $v=276 \mathrm{~cm}^{-1}$ for $A c-G G$ and $\Delta \mathrm{S}_{\mathrm{osc}}=-2.3 \mathrm{~J} \mathrm{~mol}^{-1} \mathrm{~K}^{-1}$ and $v=238 \mathrm{~cm}^{-1}$ for $S c G-$ $G$.

The above analysis confirms the key importance of the H33-S1-C20-C2 arrangement on the vibrational entropy. In the conformers with the trans arrangement of this dihedral the S-H group is able to oscillate loosely. As a result, energies of vibrations involving this group (vibrations where the contribution from the equilibrium position of the hydrogen atom H33 is the largest) are the lowest. In conformers possessing steric constraints involving the S-H group (conformers destabilized by entropy), the mercapto group oscillation is restricted and energies of the corresponding vibrations are comparatively high.

\section{Conclusions}

The conformational analysis of MEAA allowed predicting the existence of fourteen lowenergy conformers. The thermochemical values computed for these forms show that the Gibbs free energy order do not follow the zero-point energy order indicating that entropy affects the abundance of conformers (the entropy effect). The analysis of the structures of MEAA shows that the H-bond is not a factor that differentiate entropy values between conformers as the intramolecular H-bond of similar strength is predicted for all considered forms. Therefore, the side-chain arrangement affecting the steric conditions is the only factor determining entropy differences between conformers. 
A detailed analysis of entropy contributions demonstrates that the entropy effect is associated only with the differences between the rotational and vibrational entropy, with the latter factor being the predominant one. The rotational entropy depends on the product of moments of inertia and the structural feature correlated with it is mostly the conformation of the C20-C2N3-C18 chain with trans forms being stabilized compared to anticlinal or synclinal ones. Thus, the moment of inertia perpendicular to the molecular plane gives significant input to $\Delta \mathrm{S}_{\text {rot }}$

The vibrational entropy depends on vibrational frequencies and structurally is related mainly with the arrangement of the H33-S1-C20-C2 angle, with the trans conformers being considerably entropically stabilized when compared to the gauche or minus gauche ones. In accordance to that, vibrations associated with the $\tau_{\mathrm{S} 1 \mathrm{C} 20}$ coordinate has the greatest impact on vibrational entropy differences (Fig. 6).

\section{Acknowledgments}

The authors thank for the computing time to the Academic Computer Centre 'Cyfronet', Kraków, Poland.

\section{Supporting information available}

Tables S.1-S.17 and Fig. S.18 as discussed in the text. This material is available free of charge via Internet at http://pubs.asc.org. 


\section{References}

1. Brown JP, Brown JR (1976) Mutat Res 40:203-224

2. Huang Q, Lu G, Shen HM, Chung MCM, Choon NO (2007) Med Res Rev 27:609-630

3. Agarwal K, Singh SS, Verma S, Kumar S (2000) J Ethnopharmacol 72:43-46

4. Hatano T, Uebayashi H, Ito H, Shiota S, Tsuchiya T, Yoshida T (1999) Chem Pharmacol Bull 47:1121-1127

5. Montoya SCN, Comini LR, Sarmiento M, Becerra C, Albesa I, Argüello GA, Cabrera J (2005) J Photochem Photobiol B: Biol 78:77-83

6. Rajendran M, Ramasamy S, Rajamanickam C, Gandhidasan R, Murugesan R (2003) Biochim Biophys Acta 1622:65-72

7. Inbaraj JJ, Krishna MC, Gandhidasan R, Murugesan R (1999) Biochim Biophys Acta 1472:462-470

8. Reszka KJ, Bilski P, Chignell CF, Hartley JA, Khan N, Souhami RL, Mendonca AJ, Lown JWJ (1992) Photochem Photobiol Biol 15:317-335

9. Jacquemin D, Wathelet V, Preat J, Perpète EA (2007) Spectrochim Acta A 67:334-341

10. Perpète EA, Wathelet V, Preat J, Lambert C, Jacquemin D (2006) J Chem Theory Comput 2:434-440

11. Guillaumont D, Nakamura S (2000) Dyes Pigm 46:85-92

12. Avbelj F, Fele L (1998) J Mol Biol 279:665-684

13. Doig A (1996) J Biophys Chem 61:131-141

14. Kaczor A, Reva ID, Proniewicz LM, Fausto R (2006) J Phys Chem A 110:2360-2370

15. Kaczor A, Reva ID, Proniewicz LM, Fausto R (2007) J Phys Chem A 111:2957-2965

16. Frisch MJ, Trucks GW, Schlegel HB, Scuseria GE, Robb MA, Cheeseman JR, Montgomery JA, Vreven Jr,T, Kudin KN, Burant JC, Millam JM, Iyengar SS, Tomasi J, Barone V, Mennucci B, Cossi M, Scalmani G, Rega N, Petersson GA, Nakatsuji H, Hada M, Ehara M, Toyota K, Fukuda R, Hasegawa J, Ishida M, Nakajima T, Honda Y, Kitao O, Nakai H, Klene M, Li X, Knox JE, Hratchian HP, Cross JB, Bakken V, Adamo C, Jaramillo J, Gomperts R, Stratmann RE, Yazyev O, Austin AJ, Cammi R, Pomelli C, Ochterski JW, Ayala PY, Morokuma K, Voth GA, Salvador P, Dannenberg JJ, Zakrzewski VG, Dapprich S, Daniels AD, Strain MC, Farkas O, Malick DK, Rabuck AD, Raghavachari K, Foresman JB, Ortiz JV, Cui Q, Baboul AG, Clifford S, Cioslowski J, Stefanov BB, Liu G, Liashenko A, Piskorz P, Komaromi I, Martin RL, Fox DJ, Keith T, Al-Laham MA, Peng CY, Nanayakkara A, Challacombe M, Gill PMW, Johnson B, Chen 
W, Wong MW, Gonzalez C, Pople JA (2004) Gaussian 03, Revision E.01. Gaussian Inc, Wallingford CT

17. Slater JC (1974) Quantum Theory of Molecular and Solids Vol 4: The Self-Consistent Field for Molecular and Solids. McGraw-Hill, New York

18. Vosko SH, Wilk L, Nusair M (1980) Can J Phys 58:1200-1211

19. Becke AD (1988) Phys Rev A 38:3098-3100

20. Lee CT, Yang WT, Parr RG (1988) Phys Rev B 37:785-789

21. Becke AD (1993) J Chem Phys 98:5648-5652

22. Perdew JP, Wang Y (1992) Phys Rev B 45:13244-13249

23. Pulay P, Fogarasi G, Pang F, Boggs JE (1979) J Am Chem Soc 101:2550-2560

24. Martin JML, Van Alsenoy C (1995) Gar2ped. University of Antwerp

25. McQuarrie DA, Simon JD (1999) Molecular Thermodynamics. University Science Books, Sausalito California 


\section{Tables}

Table 1 Relative zero-point-corrected energies $\left(\Delta \mathrm{E}_{\text {corr }}\right)$, dipole moments $(\mu)$ and main dihedral angles of all low-energy MEAA conformers calculated at the B3LYP/6-311++G(d,p) level

\begin{tabular}{|c|c|c|c|c|c|c|c|}
\hline Conformer & $\begin{array}{c}\Delta \mathrm{E}_{\text {corr }} \\
{\left[\mathrm{kJ} \mathrm{mol}^{-1}\right]}\end{array}$ & $\stackrel{\stackrel{\mu}{ }}{[\mathrm{D}]}$ & $\begin{array}{r}\angle(\mathrm{H} 23- \\
\text { N3-C18- } \\
\left.\text { C16) [ }{ }^{\circ}\right]\end{array}$ & $\begin{array}{c}\angle(\mathrm{C} 2- \\
\text { N3-C18- } \\
\text { C7) }\left[{ }^{\circ}\right]\end{array}$ & $\begin{array}{l}\angle(\mathrm{C} 20- \\
\mathrm{C} 2-\mathrm{N3}- \\
\mathrm{C} 18)\left[^{\circ}\right]\end{array}$ & $\begin{array}{r}\angle(\mathrm{S} 1- \\
\mathrm{C} 20-\mathrm{C} 2- \\
\mathrm{N3})\left[^{\circ}\right]\end{array}$ & $\begin{array}{c}\angle(\text { H33- } \\
\text { S1-C20- } \\
\text { C2) }\left[^{\circ}\right]\end{array}$ \\
\hline$S c T-G$ & $0.00^{1}$ & 2.42 & -1.5 & 3.2 & 81.5 & -176.7 & -66.3 \\
\hline$T T-G$ & 0.24 & 1.33 & 0.8 & -0.3 & 178.7 & -178.1 & -69.0 \\
\hline ScTG & 0.65 & 1.32 & -1.0 & 0.8 & 82.8 & 177.5 & 68.3 \\
\hline$S c G-G$ & 0.88 & 2.13 & 0.5 & -0.8 & 81.9 & 64.9 & -64.2 \\
\hline$T-G G$ & 1.19 & 2.46 & 4.8 & -2.7 & 171.5 & -70.5 & 65.5 \\
\hline$T G G$ & 3.58 & 2.81 & 7.7 & -2.8 & 172.8 & 62.6 & 63.5 \\
\hline$A c-G G$ & 4.08 & 3.23 & 2.2 & -14.1 & 115.5 & -67.8 & 64.3 \\
\hline ScTT & 4.38 & 1.57 & -0.9 & 0.4 & 83.2 & -177.5 & -178.8 \\
\hline$T T T$ & 4.44 & 2.26 & 0.0 & 0.0 & 180.0 & 180.0 & 180.0 \\
\hline$S c G G$ & 4.70 & 3.39 & -3.9 & 3.7 & 80.0 & 63.9 & 71.0 \\
\hline$T G T$ & 6.41 & 3.57 & 1.8 & 1.4 & 177.6 & 66.8 & -179.8 \\
\hline$S c G T$ & 8.06 & 3.12 & -0.6 & 1.9 & 78.9 & 61.2 & 166.1 \\
\hline$A c-G-G$ & 9.07 & 3.95 & 0.4 & -6.6 & 110.5 & -66.6 & -75.9 \\
\hline$A c-G T$ & 11.5 & 4.17 & -0.2 & -6.2 & 106.8 & -67.4 & -171.7 \\
\hline
\end{tabular}

${ }^{1} \mathrm{E}_{\text {corr }}=-3205631.03\left[\mathrm{~kJ} \mathrm{~mol}^{-1}\right]$. 
Table 2 Basic thermodynamic values and abundances of all low-energy MEAA conformers calculated at the B3LYP/6-311++G(d,p) level at $25^{\circ} \mathrm{C}$

\begin{tabular}{|c|c|c|c|c|c|c|}
\hline Conformer & Degeneracy & $\begin{array}{c}\Delta \mathbf{E}_{\mathbf{c o r r}} \\
{\left[\mathbf{k J ~ m o l}^{-\mathbf{1}}\right]}\end{array}$ & $\begin{array}{c}\mathbf{\Delta G} \\
{\left[\mathbf{k J ~ m o l}^{-\mathbf{1}}\right]}\end{array}$ & $\begin{array}{c}\mathbf{\Delta H} \\
{\left[\mathbf{k J ~ m o l}^{-\mathbf{1}}\right]}\end{array}$ & $\begin{array}{c}\mathbf{T} \mathbf{\Delta} \mathbf{S}_{\text {tot }} \\
{\left[\mathbf{k J} \mathbf{~ m o l}^{\mathbf{1}}\right]}\end{array}$ & $\begin{array}{c}\text { Abundance } \\
{[\mathbf{\%}]}\end{array}$ \\
\hline$S c T-G$ & 2 & $0.00^{1}$ & $0.00^{2}$ & $0.00^{3}$ & 0.00 & 17.4 \\
\hline$T T-G$ & 2 & 0.24 & -0.68 & 0.82 & 1.50 & 22.9 \\
\hline$S c T G$ & 2 & 0.65 & 0.56 & 0.69 & 0.13 & 13.9 \\
\hline$S c G-G$ & 2 & 0.88 & 1.41 & 0.56 & -0.86 & 9.84 \\
\hline$T-G G$ & 2 & 1.19 & 0.49 & 1.27 & 0.78 & 14.3 \\
\hline$T G G$ & 2 & 3.58 & 3.76 & 3.59 & -0.17 & 3.82 \\
\hline$A c-G G$ & 2 & 4.08 & 5.05 & 3.62 & -1.44 & 2.27 \\
\hline$S c T T$ & 2 & 4.38 & 3.46 & 4.75 & 1.29 & 4.31 \\
\hline$T T T$ & 1 & 4.44 & 1.75 & 5.60 & 3.84 & 4.29 \\
\hline$S c G G$ & 2 & 4.70 & 4.74 & 4.58 & -0.15 & 2.58 \\
\hline$T G T$ & 2 & 6.41 & 4.85 & 6.99 & 2.13 & 2.46 \\
\hline$S c G T$ & 2 & 8.06 & 6.77 & 8.40 & 1.63 & 1.13 \\
\hline$A c-G-G$ & 2 & 9.07 & 8.57 & 8.98 & 0.41 & 0.55 \\
\hline$A c-G T$ & 2 & 11.5 & 10.2 & 11.8 & 1.55 & 0.28 \\
\hline
\end{tabular}

${ }^{1} \mathrm{E}_{\text {corr }}=-3205631.03\left[\mathrm{~kJ} \mathrm{~mol}^{-1}\right],{ }^{2} \mathrm{G}=-3205752.20\left[\mathrm{~kJ} \mathrm{~mol}^{-1}\right],{ }^{3} \mathrm{H}=-3205584.33\left[\mathrm{~kJ} \mathrm{~mol}{ }^{-1}\right]$ 


\section{Figure captions}

Fig. 1 Atom numbering for MEAA

Fig. 2 The optimized B3LYP/6-311++G(d,p) geometries of the most stable MEAA conformers. The values of the zero-point-corrected energy relative to the most stable conformer (in $\mathrm{kJ} \mathrm{mol}^{-1}$ ) are given in the parentheses

Fig. 3 Correlation of the calculated B3LYP/6-311++G(d,p) zero-point corrected energy $\left(\Delta \mathrm{E}_{\text {corr }}\right)$ and Gibbs free energy $\left(\Delta \mathrm{G}\right.$, both in $\mathrm{kJ} \mathrm{mol}^{-1}$, referred to the most stable conformer) for most-stable conformers of MEAA

Fig. 4 Rotational contributions to entropy $\left(\Delta \mathrm{S}_{\mathrm{rot}}\right.$, in $\mathrm{J} \mathrm{mol}^{-1} \mathrm{~K}^{-1}$ referred to the most stable conformer) (A) and z-components of the moment of inertia $\left(I_{z}\right.$, in $\left.\mathrm{kg} \mathrm{m}^{2}\right)$ for moststable conformers of MEAA (B) calculated at the B3LYP/6-311++G(d,p) level

Fig. 5 Vibrational contributions to entropy $\left(\Delta \mathrm{S}_{\mathrm{vib}}\right.$, in $\mathrm{J} \mathrm{mol}^{-1} \mathrm{~K}^{-1}$ referred to the most stable conformer) (A) and wavenumber associated with the vibration with the highest contribution from the $\tau_{\mathrm{S} 1 \mathrm{C} 20}$ mode $\left(\nu_{\tau \mathrm{S} 1 \mathrm{C} 20}\right.$, in $\left.\mathrm{cm}^{-1}\right)$ for most-stable conformers of MEAA (B) calculated at the B3LYP/6-311++G(d,p) level

Fig. 6 Rotational $\Delta \mathrm{S}_{\mathrm{rot}}$ and vibrational $\Delta \mathrm{S}_{\mathrm{vib}}$ contributions to entropy (in $\mathrm{J} \mathrm{mol}^{-1} \mathrm{~K}^{-1}$ ) correlated to the Gibbs free energy $\left(\Delta \mathrm{G}\right.$, in $\mathrm{kJ} \mathrm{mol}^{-1}$ referred to the most stable conformer) 


\section{Figure}

Click here to download high resolution image

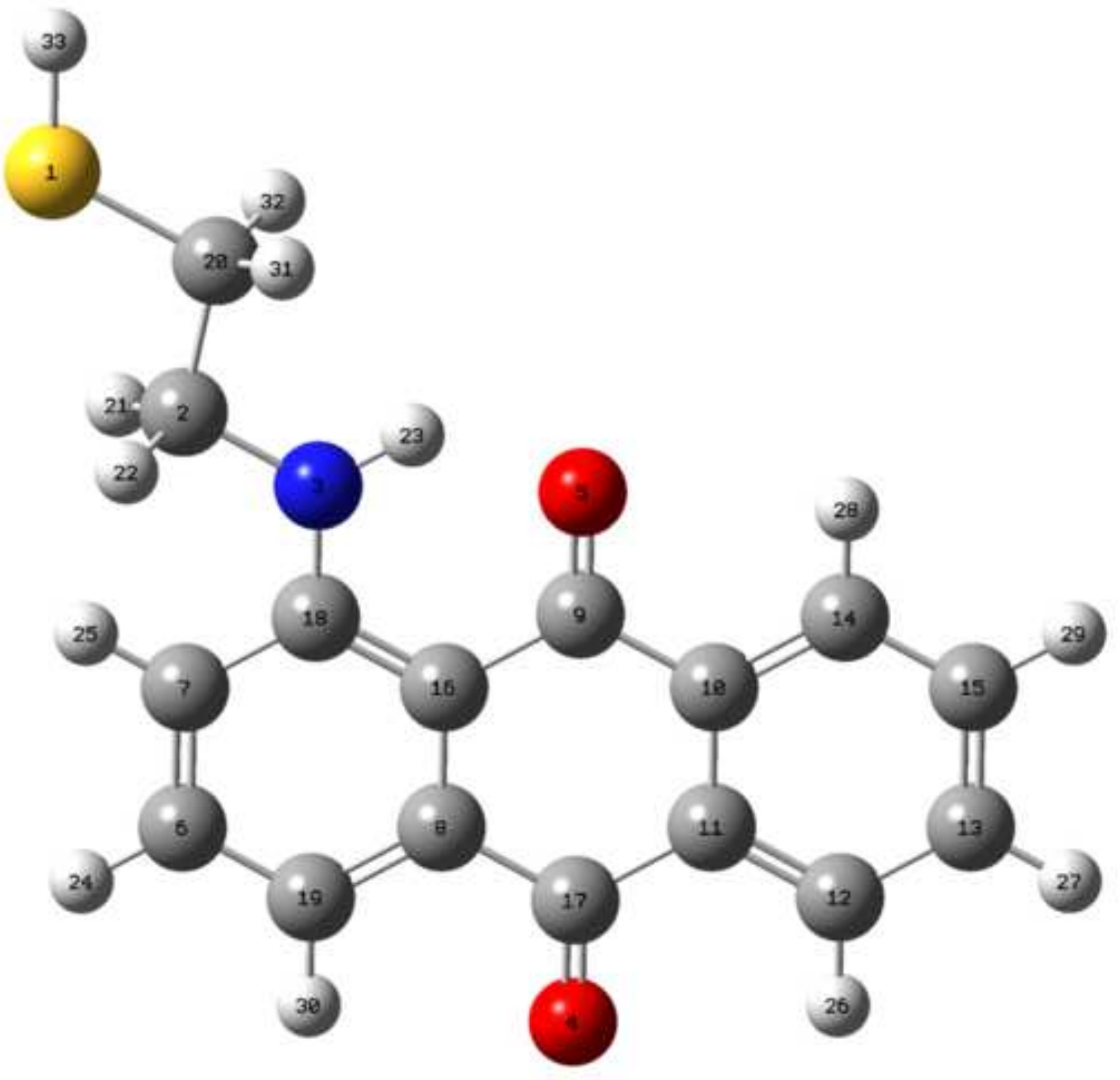


Figure 2

Click here to download high resolution image

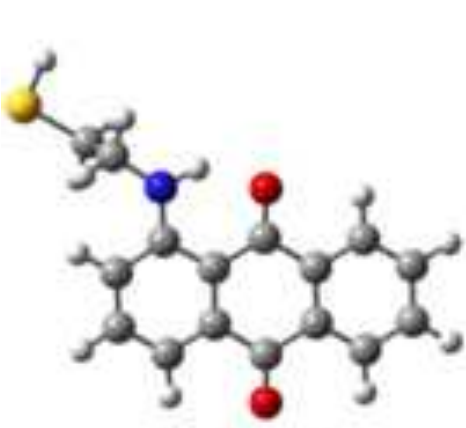

$S_{C T-G}(0.0)$

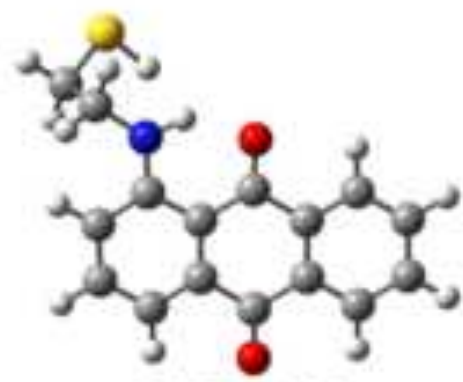

$S \subset G-G(0.9)$

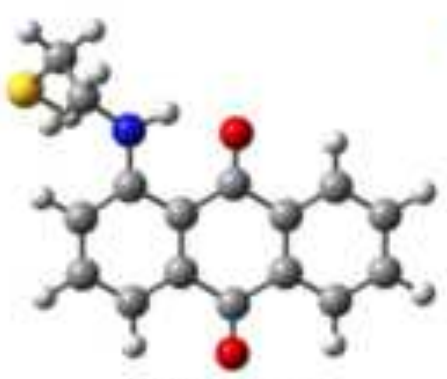

Ac-GG (4.1)

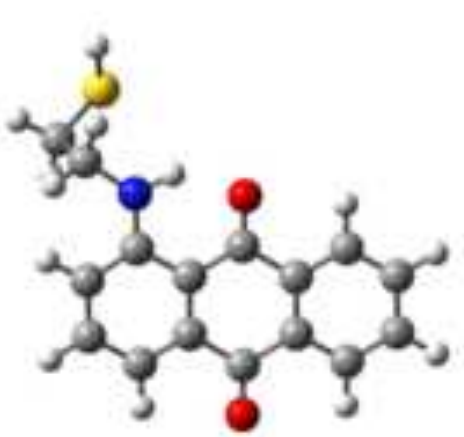

ScGG (4.7)

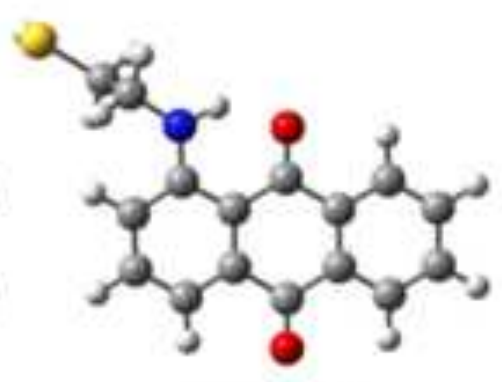

SCTT (4.4)

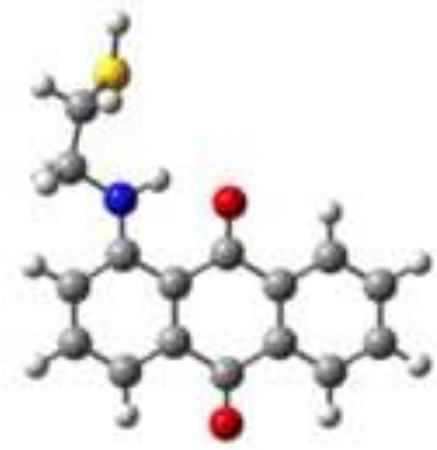

TGT (6.4)

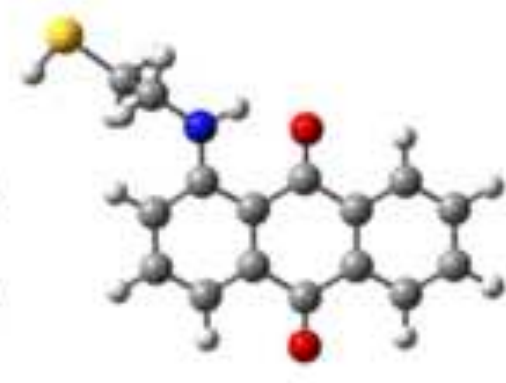

ScTG (0.7)

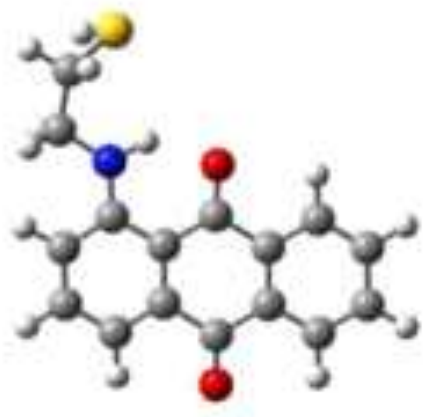

TGG (3.6)

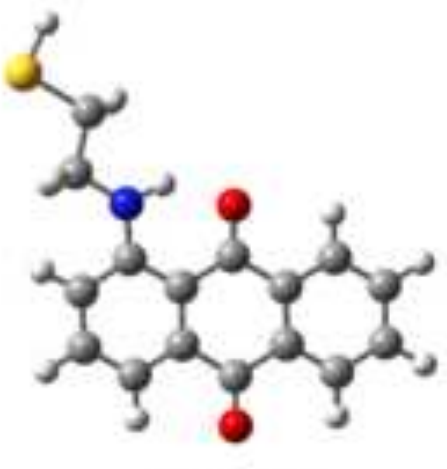

TTT (4.4)

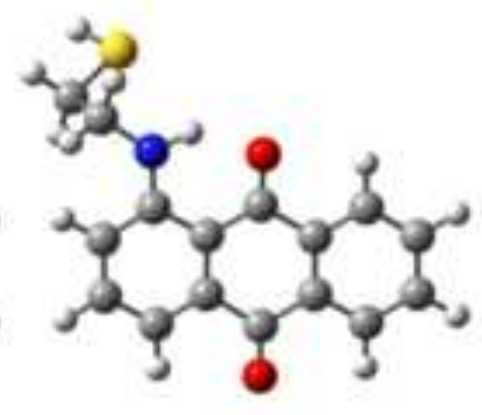

SoGT (8.1)

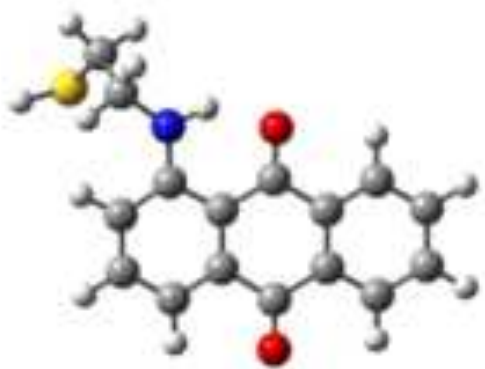

$A c-G-G(9.1)$

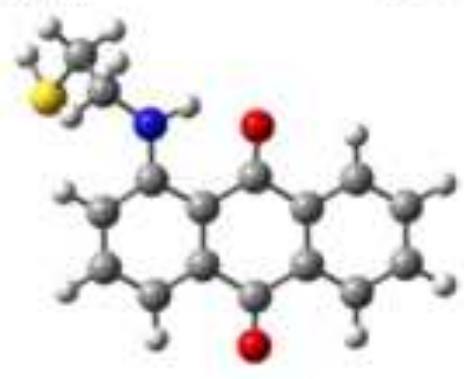

Ac-GT (11.5) 


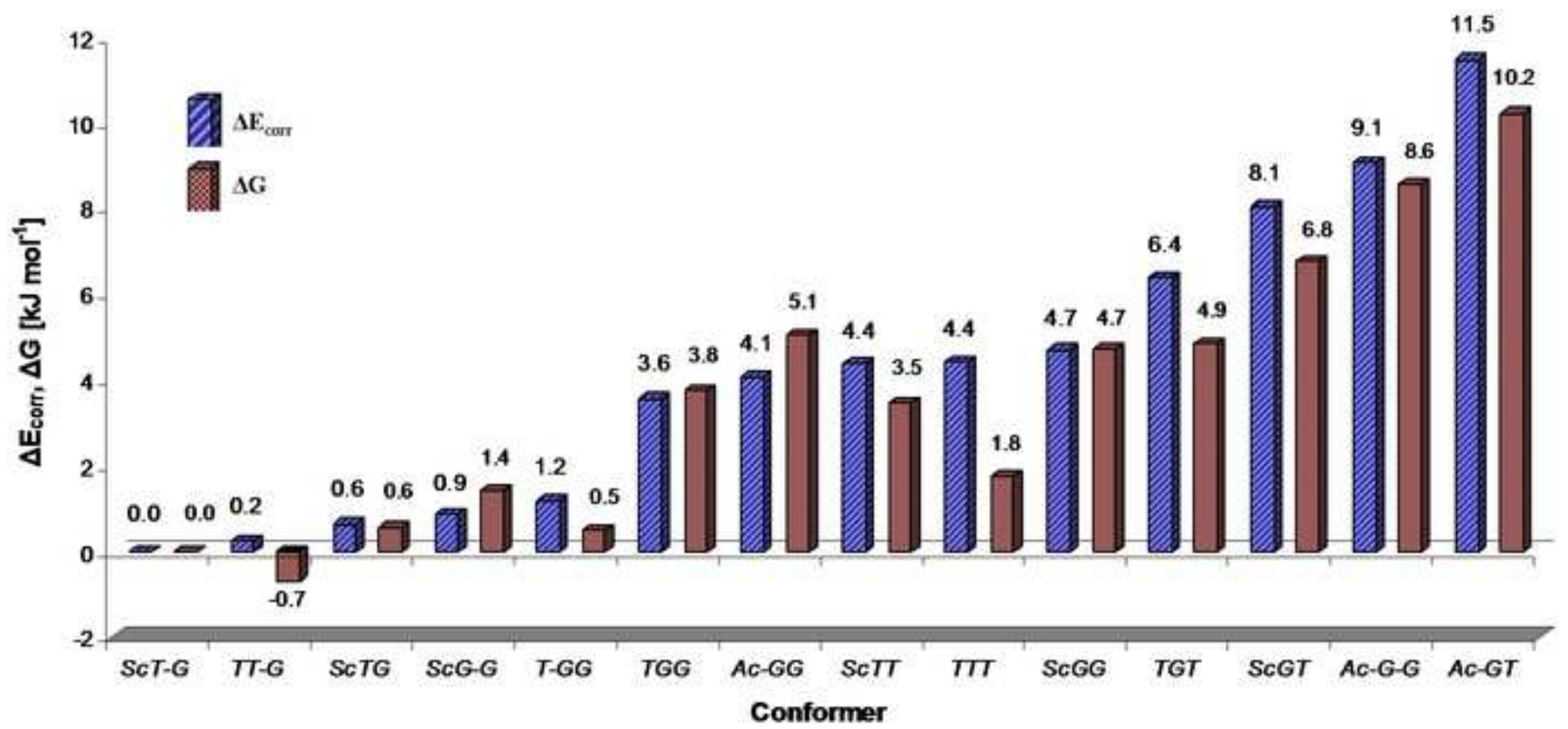


Click here to download high resolution image

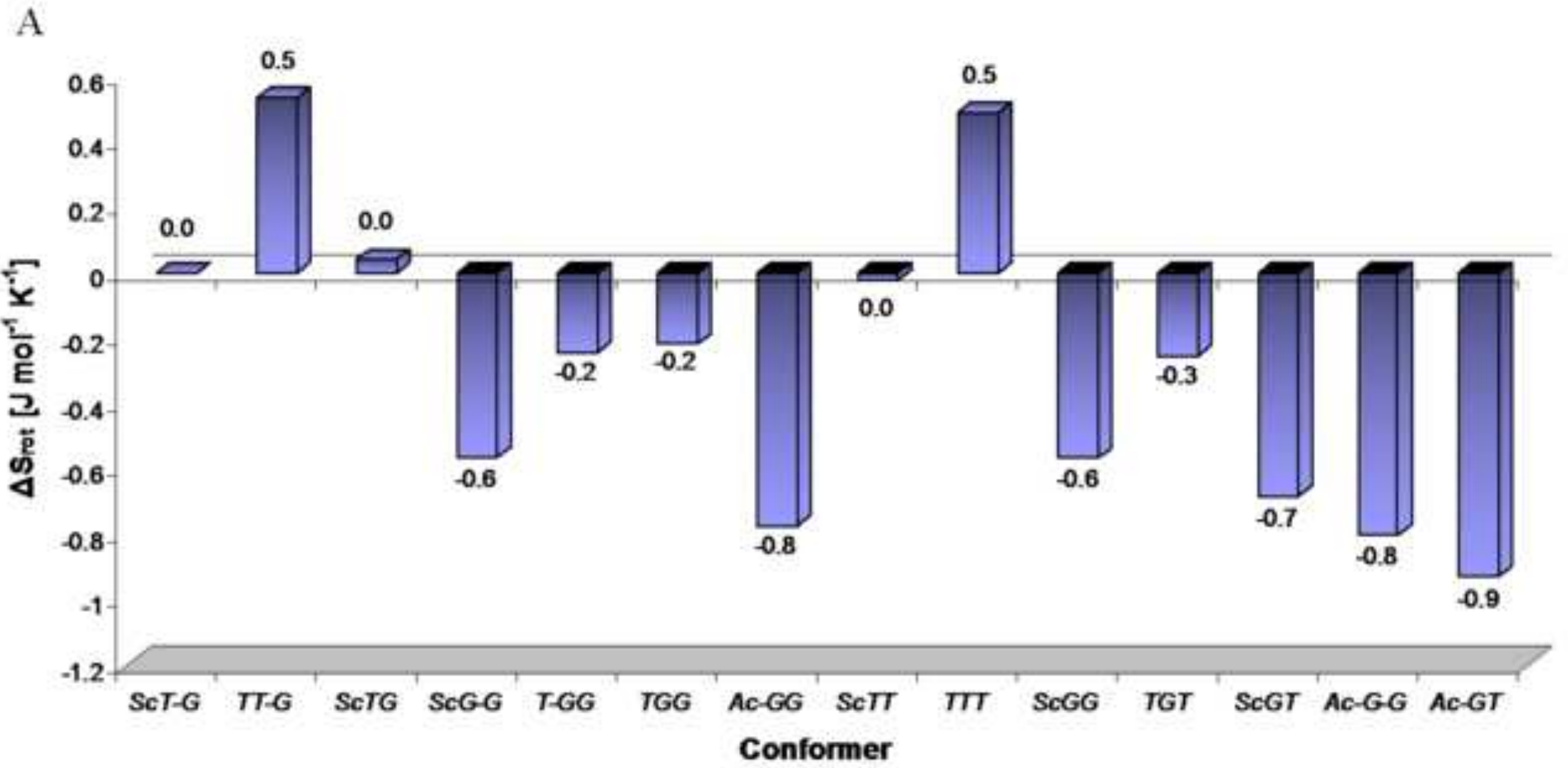

B

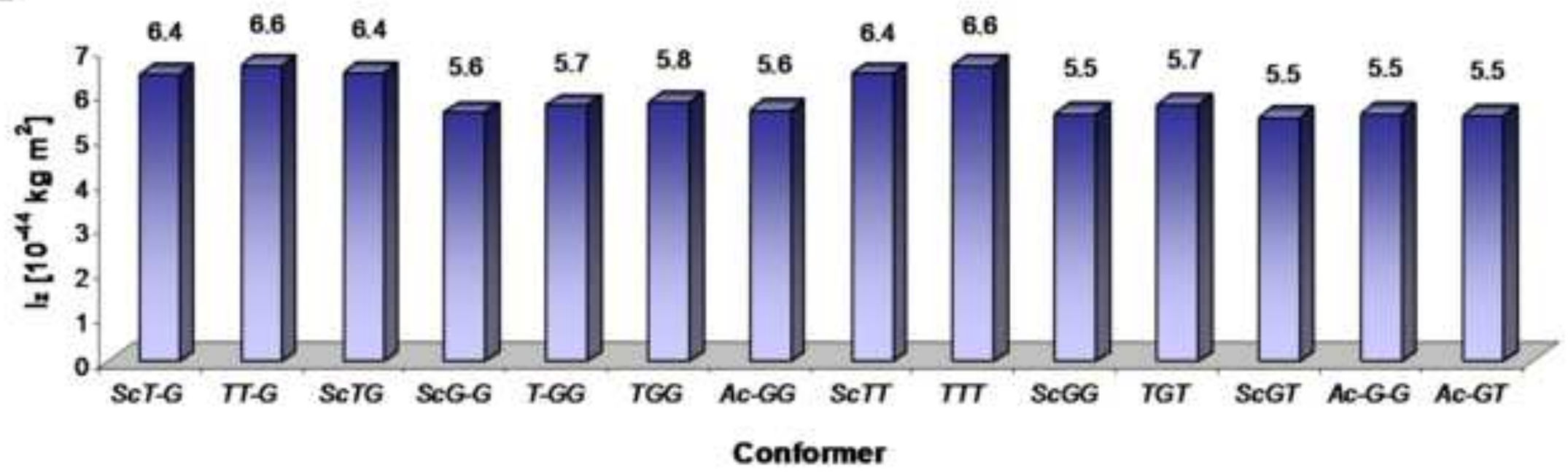


Click here to download high resolution image
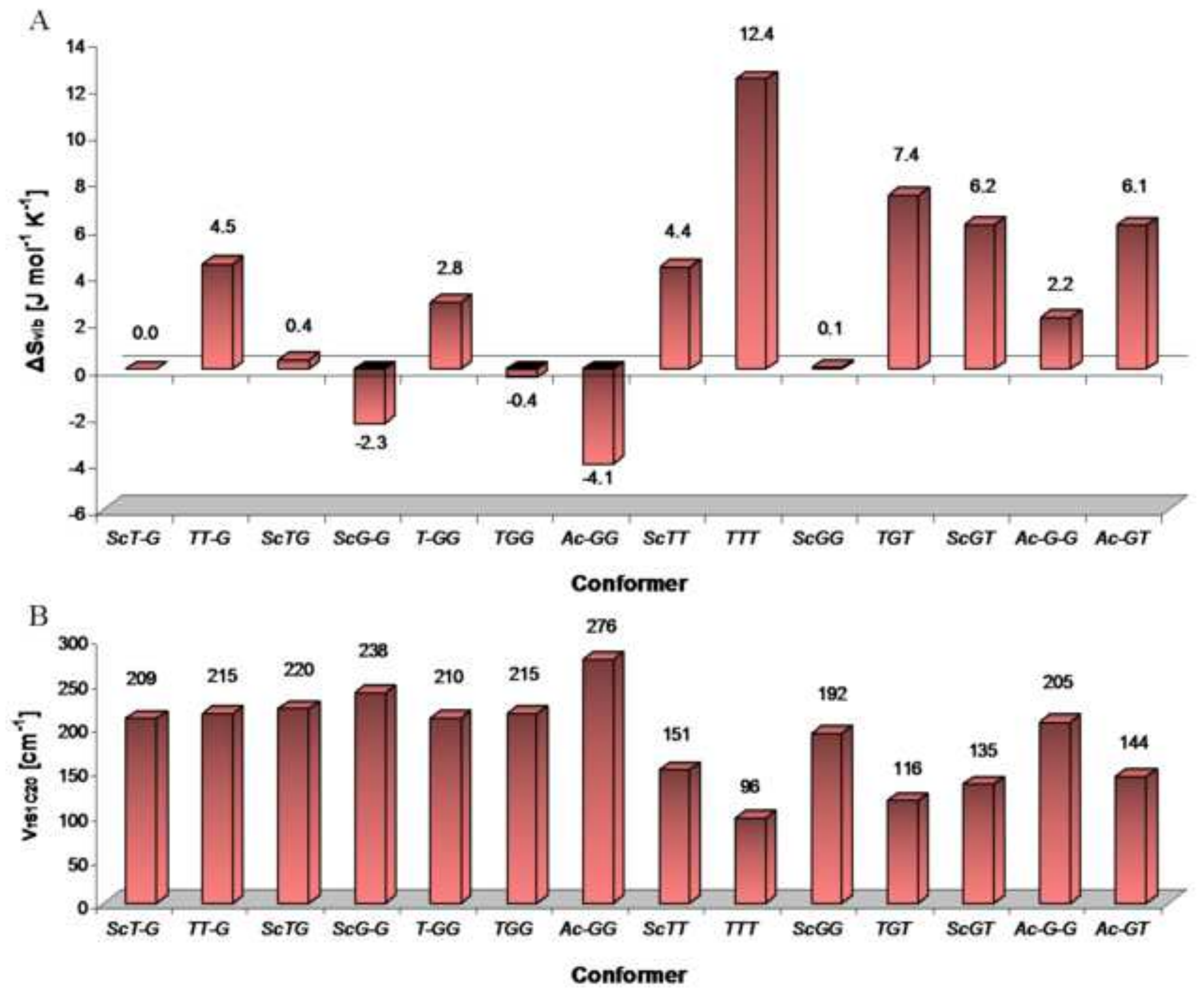
Click here to download high resolution image

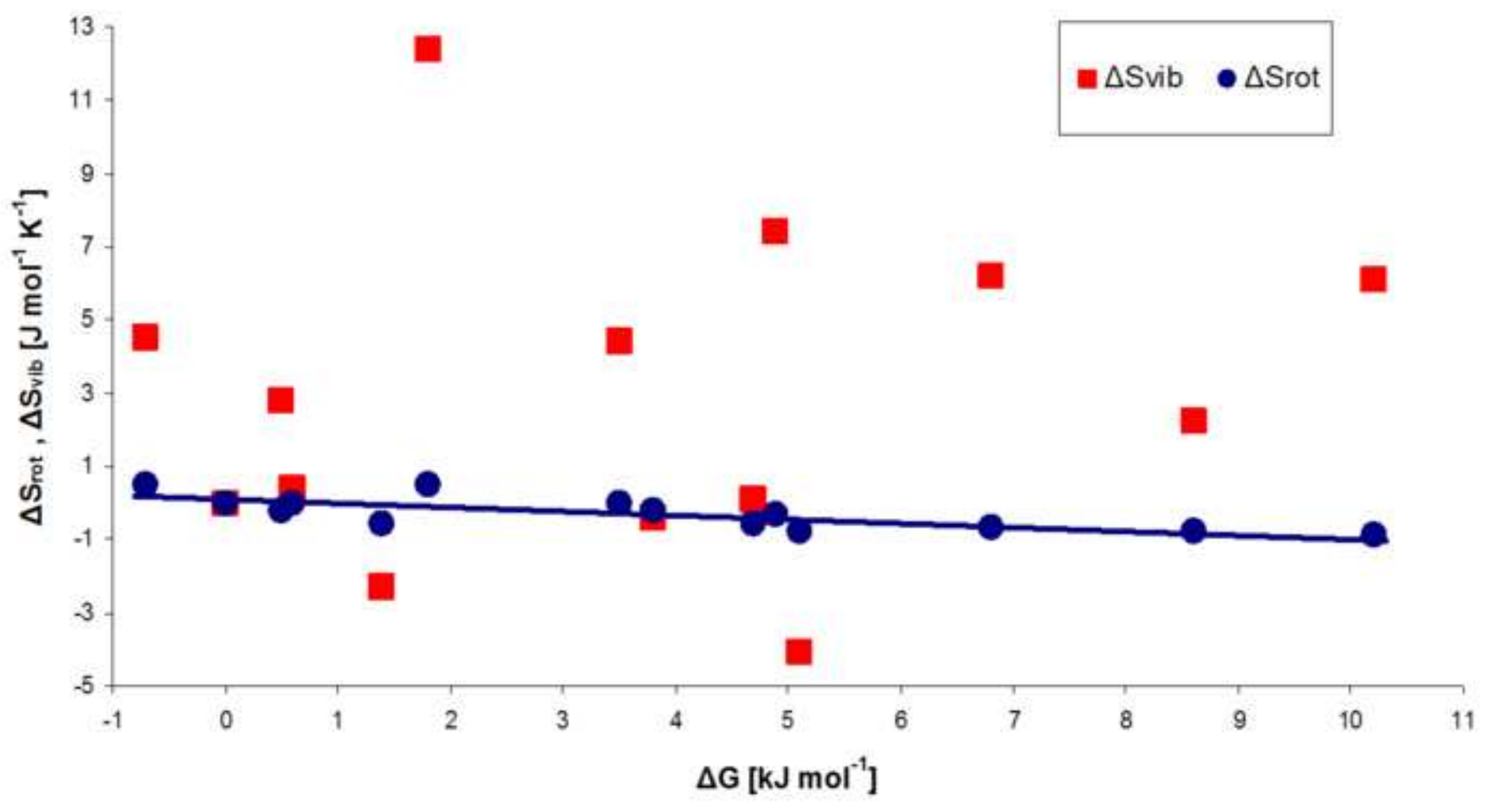


Table S.1. Geometry of the H-bonds of all low-energetic MEAA conformers calculated at the B3LYP/6-311++G(d,p).

\begin{tabular}{|c|c|c|c|c|}
\hline Conformer & $\begin{array}{c}\text { N3----O5 } \\
{[\AA ̊]}\end{array}$ & $\begin{array}{c}\text { N3-H23 } \\
\text { [̊̊] }\end{array}$ & $\begin{array}{c}\text { H23----O5 } \\
\text { [A] }\end{array}$ & $\begin{array}{c}\angle(\mathrm{N3}-\mathrm{H} 23--- \\
\text { O5) }\left[^{\circ}\right]\end{array}$ \\
\hline$S c T-G$ & 2.630 & 1.016 & 1.801 & 136.2 \\
\hline$T T-G$ & 2.631 & 1.017 & 1.807 & 135.5 \\
\hline$S c T G$ & 2.630 & 1.016 & 1.801 & 136.2 \\
\hline$S c G-G$ & 2.631 & 1.016 & 1.804 & 135.9 \\
\hline$T-G G$ & 2.628 & 1.017 & 1.801 & 135.9 \\
\hline$T G G$ & 2.637 & 1.017 & 1.832 & 133.5 \\
\hline$A c-G G$ & 2.628 & 1.018 & 1.781 & 138.1 \\
\hline ScTT & 2.629 & 1.016 & 1.801 & 136.1 \\
\hline$T T T$ & 2.631 & 1.017 & 1.810 & 135.3 \\
\hline$S c G G$ & 2.632 & 1.016 & 1.808 & 135.7 \\
\hline$T G T$ & 2.635 & 1.017 & 1.822 & 134.3 \\
\hline$S c G T$ & 2.630 & 1.016 & 1.801 & 136.1 \\
\hline$A c-G-G$ & 2.624 & 1.018 & 1.776 & 138.1 \\
\hline$A c-G T$ & 2.625 & 1.018 & 1.781 & 137.7 \\
\hline
\end{tabular}


Table S.2. Products of moments of inertia values $\left(\mathrm{I}_{\mathrm{x}} \cdot \mathrm{I}_{\mathrm{y}} \cdot \mathrm{I}_{\mathrm{z}}, 10^{11} \mathrm{u}^{3} \cdot \mathrm{bohr}^{6}\right)$ of all low-energetic MEAA conformers (calculated by using all applied methods).

\begin{tabular}{|c|c|c|c|c|c|c|}
\hline \multirow{4}{*}{ Conformer } & \multicolumn{6}{|c|}{ Method/basis set } \\
\hline & \multicolumn{5}{|c|}{ DFT } & \multirow{3}{*}{$\begin{array}{c}\text { HF } \\
6-31 G(d, p)\end{array}$} \\
\hline & \multicolumn{2}{|c|}{ B3LYP } & \multirow{2}{*}{$\begin{array}{c}\text { BLYP } \\
6-31 G(d, p)\end{array}$} & \multirow{2}{*}{$\begin{array}{c}\text { B3PW91 } \\
\text { 6-31G(d,p) }\end{array}$} & \multirow{2}{*}{\begin{tabular}{|c|} 
SVWN \\
6-31G $(d, p)$
\end{tabular}} & \\
\hline & $\begin{array}{c}6- \\
311++G(d, p)\end{array}$ & $\begin{array}{c}6- \\
31 G(d, p)\end{array}$ & & & & \\
\hline$S c T-G$ & 4.23 & 4.24 & 4.47 & 4.16 & 3.85 & 4.11 \\
\hline$T T-G$ & 4.81 & 4.83 & 5.06 & 4.74 & 4.51 & 4.67 \\
\hline ScTG & 4.27 & 4.29 & 4.52 & 4.22 & 3.91 & 4.14 \\
\hline$S c G-G$ & 3.69 & 3.66 & 3.84 & 3.60 & 3.13 & 3.61 \\
\hline$T-G G$ & 3.99 & 3.91 & 4.08 & 3.85 & 3.35 & 3.90 \\
\hline$T G G$ & 4.01 & 4.01 & 4.20 & 3.94 & 3.64 & 3.91 \\
\hline$A c-G G$ & 3.51 & 3.49 & 3.67 & 3.41 & 3.09 & 3.48 \\
\hline ScTT & 4.20 & 4.23 & 4.46 & 4.15 & 3.81 & 4.08 \\
\hline$T T T$ & 4.76 & 4.77 & 5.00 & 4.69 & 4.45 & 4.63 \\
\hline$S c G G$ & 3.69 & 3.66 & 3.85 & 3.59 & 3.25 & 3.57 \\
\hline$T G T$ & 3.98 & 3.94 & 4.10 & 3.87 & 3.53 & 3.87 \\
\hline ScGT & 3.59 & 3.55 & 3.74 & 3.49 & 3.05 & 3.47 \\
\hline$A c-G-G$ & 3.49 & 3.47 & 3.65 & 3.39 & 3.09 & 3.45 \\
\hline$A c-G T$ & 3.38 & 3.37 & 3.54 & 3.31 & 3.02 & 3.36 \\
\hline
\end{tabular}


Table S.3. Definitions of internal coordinates for MEAA molecule.

\begin{tabular}{|c|c|c|}
\hline $\mathbf{N r}$ & Definition of internal coordinate & Symbol \\
\hline 1 & $v(\mathrm{~S} 1 \mathrm{C} 20)$ & $v_{\mathrm{S} 1 \mathrm{C} 20}$ \\
\hline 2 & $v(\mathrm{~S} 1 \mathrm{H} 33)$ & $v_{\mathrm{S} 1 \mathrm{H} 33}$ \\
\hline 3 & $v(\mathrm{C} 2 \mathrm{~N} 3)$ & $v_{\mathrm{C} 2 \mathrm{~N} 3}$ \\
\hline 4 & $v(\mathrm{C} 2 \mathrm{C} 20)$ & $v_{\mathrm{C} 2 \mathrm{C} 20}$ \\
\hline 5 & $v(\mathrm{C} 2 \mathrm{H} 21)$ & $v_{\mathrm{C} 2 \mathrm{H} 21}$ \\
\hline 6 & $v(\mathrm{C} 2 \mathrm{H} 22)$ & $v_{\mathrm{C} 2 \mathrm{H} 22}$ \\
\hline 7 & $v(\mathrm{~N} 3 \mathrm{C} 18)$ & $v_{\mathrm{N} 3 \mathrm{C} 18}$ \\
\hline 8 & $v(\mathrm{~N} 3 \mathrm{H} 23)$ & $v_{\mathrm{N} 3 \mathrm{H} 23}$ \\
\hline 9 & $v(\mathrm{O} 4 \mathrm{C} 17)$ & $v_{\mathrm{O} 4 \mathrm{C} 17}$ \\
\hline 10 & $v(\mathrm{O} 5 \mathrm{C} 9)$ & $v_{\mathrm{O} 5 \mathrm{C} 9}$ \\
\hline 11 & $v(\mathrm{C} 6 \mathrm{C} 7)$ & $v_{\mathrm{C} 6 \mathrm{C} 7}$ \\
\hline 12 & $v(\mathrm{C} 6 \mathrm{C} 19)$ & $v_{\mathrm{C} 6 \mathrm{C} 19}$ \\
\hline 13 & $v(\mathrm{C} 6 \mathrm{H} 24)$ & $v_{\mathrm{C} 6 \mathrm{H} 24}$ \\
\hline 14 & $v(\mathrm{C} 7 \mathrm{C} 18)$ & $v_{\mathrm{C} 7 \mathrm{C} 18}$ \\
\hline 15 & $v(\mathrm{C} 7 \mathrm{H} 25)$ & $v_{\mathrm{C} 7 \mathrm{H} 25}$ \\
\hline 16 & $v(\mathrm{C} 8 \mathrm{C} 16)$ & $v_{\mathrm{C} 8 \mathrm{C} 16}$ \\
\hline 17 & $v(\mathrm{C} 8 \mathrm{C} 17)$ & $v_{\mathrm{C} 8 \mathrm{C} 17}$ \\
\hline 18 & $v(\mathrm{C} 8 \mathrm{C} 19)$ & $v_{\mathrm{C} 8 \mathrm{C} 19}$ \\
\hline 19 & $v(\mathrm{C} 9 \mathrm{C} 10)$ & $v_{\mathrm{C} 9 \mathrm{C} 10}$ \\
\hline 20 & $v(\mathrm{C} 9 \mathrm{C} 16)$ & $v_{\mathrm{C} 9 \mathrm{C} 16}$ \\
\hline 21 & $v(\mathrm{C} 10 \mathrm{C} 11)$ & $v_{\mathrm{C} 10 \mathrm{C} 11}$ \\
\hline 22 & $v(\mathrm{C} 10 \mathrm{C} 14)$ & $v_{\mathrm{C} 10 \mathrm{C} 14}$ \\
\hline 23 & $v(\mathrm{C} 11 \mathrm{C} 12)$ & $v_{\mathrm{C} 11 \mathrm{C} 12}$ \\
\hline 24 & $v(\mathrm{C} 11 \mathrm{C} 17)$ & $v_{\mathrm{C} 11 \mathrm{C} 17}$ \\
\hline 25 & $v(\mathrm{C} 12 \mathrm{C} 13)$ & $v_{\mathrm{C}_{12} \mathrm{C}_{13}}$ \\
\hline 26 & $v(\mathrm{C} 12 \mathrm{H} 26)$ & $v_{\mathrm{C} 12 \mathrm{H} 26}$ \\
\hline 27 & $v(\mathrm{C} 13 \mathrm{C} 15)$ & $v_{\mathrm{C} 13 \mathrm{C} 15}$ \\
\hline 28 & $v(\mathrm{C} 13 \mathrm{H} 27)$ & $v_{\mathrm{C} 13 \mathrm{H} 27}$ \\
\hline 29 & $v(\mathrm{C} 14 \mathrm{C} 15)$ & $v_{\mathrm{C} 14 \mathrm{C} 15}$ \\
\hline 30 & $v(\mathrm{C} 14 \mathrm{H} 28)$ & $v_{\mathrm{C} 14 \mathrm{H} 28}$ \\
\hline 31 & $v(\mathrm{C} 15 \mathrm{H} 29)$ & $v_{\mathrm{C} 15 \mathrm{H} 29}$ \\
\hline 32 & $v(\mathrm{C} 16 \mathrm{C} 18)$ & $v_{\mathrm{C} 16 \mathrm{C} 18}$ \\
\hline 33 & $v(\mathrm{C} 19 \mathrm{H} 30)$ & $v_{\mathrm{C} 19 \mathrm{H} 30}$ \\
\hline 34 & $v(\mathrm{C} 20 \mathrm{H} 31)$ & $v_{\mathrm{C} 20 \mathrm{H} 31}$ \\
\hline 35 & $v(\mathrm{C} 20 \mathrm{H} 32)$ & $v_{\mathrm{C} 20 \mathrm{H} 32}$ \\
\hline 36 & $5 \beta(\mathrm{H} 22 \mathrm{H} 21 \mathrm{C} 2)+\beta(\mathrm{N} 3 \mathrm{C} 20 \mathrm{C} 2)$ & $\delta_{1 / \mathrm{C} 2 \mathrm{H} 22 \mathrm{H} 21}$ \\
\hline 37 & $\beta(\mathrm{H} 22 \mathrm{H} 21 \mathrm{C} 2)+5 \beta(\mathrm{N} 3 \mathrm{C} 20 \mathrm{C} 2)$ & $\delta_{2 / \mathrm{C} 2 \mathrm{H} 22 \mathrm{H} 21}$ \\
\hline 38 & $\beta(\mathrm{N} 3 \mathrm{H} 22 \mathrm{C} 2)-\beta(\mathrm{N} 3 \mathrm{H} 21 \mathrm{C} 2)+\beta(\mathrm{C} 2 \mathrm{OH} 22 \mathrm{C} 2)-\beta(\mathrm{C} 2 \mathrm{OH} 21 \mathrm{C} 2)$ & $\rho_{\mathrm{C} 2 \mathrm{H} 22 \mathrm{H} 21}$ \\
\hline 39 & $\beta(\mathrm{N} 3 \mathrm{H} 22 \mathrm{C} 2)+\beta(\mathrm{N} 3 \mathrm{H} 21 \mathrm{C} 2)-\beta(\mathrm{C} 20 \mathrm{H} 22 \mathrm{C} 2)-\beta(\mathrm{C} 2 \mathrm{OH} 21 \mathrm{C} 2)$ & $\omega_{\mathrm{C} 2 \mathrm{H} 22 \mathrm{H} 21}$ \\
\hline 40 & $\beta(\mathrm{N} 3 \mathrm{H} 22 \mathrm{C} 2)-\beta(\mathrm{N} 3 \mathrm{H} 21 \mathrm{C} 2)-\beta(\mathrm{C} 20 \mathrm{H} 22 \mathrm{C} 2)+\beta(\mathrm{C} 2 \mathrm{HH} 21 \mathrm{C} 2)$ & $\tau_{\mathrm{C} 2 \mathrm{H} 22 \mathrm{H} 21}$ \\
\hline 41 & $5 \beta(\mathrm{H} 31 \mathrm{H} 32 \mathrm{C} 20)+\beta(\mathrm{C} 2 \mathrm{~S} 1 \mathrm{C} 20)$ & $\delta_{1 / \mathrm{C} 20 \mathrm{H} 31 \mathrm{H} 32}$ \\
\hline 42 & $\beta(\mathrm{H} 31 \mathrm{H} 32 \mathrm{C} 20)+5 \beta(\mathrm{C} 2 \mathrm{~S} 1 \mathrm{C} 20)$ & $\delta_{2 / \mathrm{C} 20 \mathrm{H} 31 \mathrm{H} 32}$ \\
\hline 43 & $\beta(\mathrm{C} 2 \mathrm{H} 31 \mathrm{C} 20)-\beta(\mathrm{C} 2 \mathrm{H} 32 \mathrm{C} 20)+\beta(\mathrm{S} 1 \mathrm{H} 31 \mathrm{C} 20)-\beta(\mathrm{S} 1 \mathrm{H} 32 \mathrm{C} 20)$ & $\rho_{\mathrm{C} 20 \mathrm{H} 31 \mathrm{H} 32}$ \\
\hline 44 & $\beta(\mathrm{C} 2 \mathrm{H} 31 \mathrm{C} 20)+\beta(\mathrm{C} 2 \mathrm{H} 32 \mathrm{C} 20)-\beta(\mathrm{S} 1 \mathrm{H} 31 \mathrm{C} 20)-\beta(\mathrm{S} 1 \mathrm{H} 32 \mathrm{C} 20)$ & $\omega_{\mathrm{C} 20 \mathrm{H} 31 \mathrm{H} 32}$ \\
\hline 45 & $\beta(\mathrm{C} 2 \mathrm{H} 31 \mathrm{C} 20)-\beta(\mathrm{C} 2 \mathrm{H} 32 \mathrm{C} 20)-\beta(\mathrm{S} 1 \mathrm{H} 31 \mathrm{C} 20)+\beta(\mathrm{S} 1 \mathrm{H} 32 \mathrm{C} 20)$ & $\tau_{\mathrm{C} 20 \mathrm{H} 31 \mathrm{H} 32}$ \\
\hline 46 & $2 \beta(\mathrm{C} 2 \mathrm{C} 18 \mathrm{~N} 3)-\beta(\mathrm{C} 2 \mathrm{H} 23 \mathrm{~N} 3)-\beta(\mathrm{C} 18 \mathrm{H} 23 \mathrm{~N} 3)$ & $\beta_{\mathrm{C} 18 \mathrm{~N} 3 \mathrm{C} 2}$ \\
\hline
\end{tabular}




\begin{tabular}{|c|c|c|}
\hline 47 & $\beta(\mathrm{C} 2 \mathrm{H} 23 \mathrm{~N} 3)-\beta(\mathrm{C} 18 \mathrm{H} 23 \mathrm{~N} 3)$ & $\rho_{\mathrm{N} 3 \mathrm{H} 23}$ \\
\hline 48 & $\gamma(\mathrm{H} 23 \mathrm{C} 2 \mathrm{C} 18 \mathrm{~N} 3)$ & $\omega_{\mathrm{N} 3 \mathrm{H} 23}$ \\
\hline 49 & $\beta(\mathrm{C} 20 \mathrm{H} 33 \mathrm{~S} 1)$ & $\beta_{\mathrm{C} 20 \mathrm{~S} 1 \mathrm{H} 33}$ \\
\hline 50 & $\beta(\mathrm{C} 6 \mathrm{C} 18 \mathrm{C} 7)-\beta(\mathrm{C} 7 \mathrm{C} 16 \mathrm{C} 18)+\beta(\mathrm{C} 18 \mathrm{C} 8 \mathrm{C} 16)-\beta(\mathrm{C} 16 \mathrm{C} 19 \mathrm{C} 8)+\beta(\mathrm{C} 8 \mathrm{C} 6 \mathrm{C} 19)-\beta(\mathrm{C} 19 \mathrm{C} 7 \mathrm{C} 6)$ & ${ }^{1} \beta_{\text {trig/R6 }}$ \\
\hline 51 & $\begin{array}{l}2 \beta(\mathrm{C} 6 \mathrm{C} 18 \mathrm{C} 7)-\beta(\mathrm{C} 7 \mathrm{C} 16 \mathrm{C} 18)-\beta(\mathrm{C} 18 \mathrm{C} 8 \mathrm{C} 16)+2 \beta(\mathrm{C} 16 \mathrm{C} 19 \mathrm{C} 8)-\beta(\mathrm{C} 8 \mathrm{C} 6 \mathrm{C} 19)- \\
\beta(\mathrm{C} 19 \mathrm{C} 7 \mathrm{C} 6)\end{array}$ & ${ }^{1} \beta_{2 / R 6}$ \\
\hline 52 & $\beta(\mathrm{C} 7 \mathrm{C} 16 \mathrm{C} 18)-\beta(\mathrm{C} 18 \mathrm{C} 8 \mathrm{C} 16)+\beta(\mathrm{C} 8 \mathrm{C} 6 \mathrm{C} 19)-\beta(\mathrm{C} 19 \mathrm{C} 7 \mathrm{C} 6)$ & ${ }^{1} \beta_{3 / R 6}$ \\
\hline 53 & $\begin{array}{l}\tau(\mathrm{C} 6 \mathrm{C} 7 \mathrm{C} 18 \mathrm{C} 16)-\tau(\mathrm{C} 7 \mathrm{C} 18 \mathrm{C} 16 \mathrm{C} 8)+\tau(\mathrm{C} 18 \mathrm{C} 16 \mathrm{C} 8 \mathrm{C} 19)-\tau(\mathrm{C} 16 \mathrm{C} 8 \mathrm{C} 19 \mathrm{C} 6)+\tau(\mathrm{C} 8 \mathrm{C} 19 \mathrm{C} 6 \mathrm{C} 7) \\
-\tau(\mathrm{C} 19 \mathrm{C} 6 \mathrm{C} 7 \mathrm{C} 18)\end{array}$ & ${ }^{1} \tau_{1 / R 6}$ \\
\hline 54 & $\tau(\mathrm{C} 6 \mathrm{C} 7 \mathrm{C} 18 \mathrm{C} 16)-\tau(\mathrm{C} 18 \mathrm{C} 16 \mathrm{C} 8 \mathrm{C} 19)+\tau(\mathrm{C} 16 \mathrm{C} 8 \mathrm{C} 19 \mathrm{C} 6)-\tau(\mathrm{C} 19 \mathrm{C} 6 \mathrm{C} 7 \mathrm{C} 18)$ & ${ }^{1} \tau_{2 / R 6}$ \\
\hline 55 & $\begin{array}{l}-\tau(\mathrm{C} 6 \mathrm{C} 7 \mathrm{C} 18 \mathrm{C} 16)+2 \tau(\mathrm{C} 7 \mathrm{C} 18 \mathrm{C} 16 \mathrm{C} 8)-\tau(\mathrm{C} 18 \mathrm{C} 16 \mathrm{C} 8 \mathrm{C} 19)-\tau(\mathrm{C} 16 \mathrm{C} 8 \mathrm{C} 19 \mathrm{C} 6) \\
+2 \tau(\mathrm{C} 8 \mathrm{C} 19 \mathrm{C} 6 \mathrm{C} 7)-\tau(\mathrm{C} 19 \mathrm{C} 6 \mathrm{C} 7 \mathrm{C} 18)\end{array}$ & ${ }^{1} \tau_{3 / R 6}$ \\
\hline 56 & $\begin{array}{l}\beta(\mathrm{C} 8 \mathrm{C} 9 \mathrm{C} 16)-\beta(\mathrm{C} 16 \mathrm{C} 10 \mathrm{C} 9)+\beta(\mathrm{C} 9 \mathrm{C} 11 \mathrm{C} 10)-\beta(\mathrm{C} 10 \mathrm{C} 17 \mathrm{C} 11)+\beta(\mathrm{C} 11 \mathrm{C} 8 \mathrm{C} 17)- \\
\beta(\mathrm{C} 17 \mathrm{C} 16 \mathrm{C} 8)\end{array}$ & ${ }^{2} \beta_{\text {trig/R6 }}$ \\
\hline 57 & $\begin{array}{l}2 \beta(\mathrm{C} 8 \mathrm{C} 9 \mathrm{C} 16)-\beta(\mathrm{C} 16 \mathrm{C} 10 \mathrm{C} 9)-\beta(\mathrm{C} 9 \mathrm{C} 11 \mathrm{C} 10)+2 \beta(\mathrm{C} 10 \mathrm{C} 17 \mathrm{C} 11)-\beta(\mathrm{C} 11 \mathrm{C} 8 \mathrm{C} 17)- \\
\beta(\mathrm{C} 17 \mathrm{C} 16 \mathrm{C} 8)\end{array}$ & ${ }^{2} \beta_{2 / R 6}$ \\
\hline 58 & $\beta(\mathrm{C} 16 \mathrm{C} 10 \mathrm{C} 9)-\beta(\mathrm{C} 9 \mathrm{C} 11 \mathrm{C} 10)+\beta(\mathrm{C} 11 \mathrm{C} 8 \mathrm{C} 17)-\beta(\mathrm{C} 17 \mathrm{C} 16 \mathrm{C} 8)$ & ${ }^{2} \beta_{3 / R 6}$ \\
\hline 59 & $\begin{array}{l}\tau(\mathrm{C} 8 \mathrm{C} 16 \mathrm{C} 9 \mathrm{C} 10)-\tau(\mathrm{C} 16 \mathrm{C} 9 \mathrm{C} 10 \mathrm{C} 11)+\tau(\mathrm{C} 9 \mathrm{C} 10 \mathrm{C} 11 \mathrm{C} 17)-\tau(\mathrm{C} 10 \mathrm{C} 11 \mathrm{C} 17 \mathrm{C} 8) \\
+\tau(\mathrm{C} 11 \mathrm{C} 17 \mathrm{C} 8 \mathrm{C} 16)-\tau(\mathrm{C} 17 \mathrm{C} 8 \mathrm{C} 16 \mathrm{C} 9)\end{array}$ & ${ }^{2} \tau_{1 / R 6}$ \\
\hline 60 & $\tau(\mathrm{C} 8 \mathrm{C} 16 \mathrm{C} 9 \mathrm{C} 10)-\tau(\mathrm{C} 9 \mathrm{C} 10 \mathrm{C} 11 \mathrm{C} 17)+\tau(\mathrm{C} 10 \mathrm{C} 11 \mathrm{C} 17 \mathrm{C} 8)-\tau(\mathrm{C} 17 \mathrm{C} 8 \mathrm{C} 16 \mathrm{C} 9)$ & ${ }^{2} \tau_{2 / R 6}$ \\
\hline 61 & $\begin{array}{l}-\tau(\mathrm{C} 8 \mathrm{C} 16 \mathrm{C} 9 \mathrm{C} 10)+2 \tau(\mathrm{C} 16 \mathrm{C} 9 \mathrm{C} 10 \mathrm{C} 11)-\tau(\mathrm{C} 9 \mathrm{C} 10 \mathrm{C} 11 \mathrm{C} 17)-\tau(\mathrm{C} 10 \mathrm{C} 11 \mathrm{C} 17 \mathrm{C} 8) \\
+2 \tau(\mathrm{C} 11 \mathrm{C} 17 \mathrm{C} 8 \mathrm{C} 16)-\tau(\mathrm{C} 17 \mathrm{C} 8 \mathrm{C} 16 \mathrm{C} 9)\end{array}$ & ${ }^{2} \tau_{3 / R 6}$ \\
\hline 62 & $\begin{array}{l}\beta(\mathrm{C} 11 \mathrm{C} 14 \mathrm{C} 10)-\beta(\mathrm{C} 10 \mathrm{C} 15 \mathrm{C} 14)+\beta(\mathrm{C} 14 \mathrm{C} 13 \mathrm{C} 15)-\beta(\mathrm{C} 15 \mathrm{C} 12 \mathrm{C} 13)+\beta(\mathrm{C} 13 \mathrm{C} 11 \mathrm{C} 12)- \\
\beta(\mathrm{C} 12 \mathrm{C} 10 \mathrm{C} 11)\end{array}$ & ${ }^{3} \beta_{\text {trig/R6 }}$ \\
\hline 63 & $\begin{array}{l}2 \beta(\mathrm{C} 11 \mathrm{C} 14 \mathrm{C} 10)-\beta(\mathrm{C} 10 \mathrm{C} 15 \mathrm{C} 14)-\beta(\mathrm{C} 14 \mathrm{C} 13 \mathrm{C} 15)+2 \beta(\mathrm{C} 15 \mathrm{C} 12 \mathrm{C} 13)-\beta(\mathrm{C} 13 \mathrm{C} 11 \mathrm{C} 12)- \\
\beta(\mathrm{C} 12 \mathrm{C} 10 \mathrm{C} 11)\end{array}$ & ${ }^{3} \beta_{2 / R 6}$ \\
\hline 64 & $\beta(\mathrm{C} 10 \mathrm{C} 15 \mathrm{C} 14)-\beta(\mathrm{C} 14 \mathrm{C} 13 \mathrm{C} 15)+\beta(\mathrm{C} 13 \mathrm{C} 11 \mathrm{C} 12)-\beta(\mathrm{C} 12 \mathrm{C} 10 \mathrm{C} 11)$ & ${ }^{3} \beta_{3 / R 6}$ \\
\hline 65 & $\begin{array}{l}\tau(\mathrm{C} 11 \mathrm{C} 10 \mathrm{C} 14 \mathrm{C} 15)-\tau(\mathrm{C} 10 \mathrm{C} 14 \mathrm{C} 15 \mathrm{C} 13)+\tau(\mathrm{C} 14 \mathrm{C} 15 \mathrm{C} 13 \mathrm{C} 12)-\tau(\mathrm{C} 15 \mathrm{C} 13 \mathrm{C} 12 \mathrm{C} 11) \\
+\tau(\mathrm{C} 13 \mathrm{C} 12 \mathrm{C} 11 \mathrm{C} 10)-\tau(\mathrm{C} 12 \mathrm{C} 11 \mathrm{C} 10 \mathrm{C} 14)\end{array}$ & ${ }^{3} \tau_{1 / R 6}$ \\
\hline 66 & $\tau(\mathrm{C} 11 \mathrm{C} 10 \mathrm{C} 14 \mathrm{C} 15)-\tau(\mathrm{C} 14 \mathrm{C} 15 \mathrm{C} 13 \mathrm{C} 12)+\tau(\mathrm{C} 15 \mathrm{C} 13 \mathrm{C} 12 \mathrm{C} 11)-\tau(\mathrm{C} 12 \mathrm{C} 11 \mathrm{C} 10 \mathrm{C} 14)$ & ${ }^{3} \tau_{2 / R 6}$ \\
\hline 67 & $\begin{array}{l}-\tau(\mathrm{C} 11 \mathrm{C} 10 \mathrm{C} 14 \mathrm{C} 15)+2 \tau(\mathrm{C} 10 \mathrm{C} 14 \mathrm{C} 15 \mathrm{C} 13)-\tau(\mathrm{C} 14 \mathrm{C} 15 \mathrm{C} 13 \mathrm{C} 12)-\tau(\mathrm{C} 15 \mathrm{C} 13 \mathrm{C} 12 \mathrm{C} 11) \\
+2 \tau(\mathrm{C} 13 \mathrm{C} 12 \mathrm{C} 11 \mathrm{C} 10)-\tau(\mathrm{C} 12 \mathrm{C} 11 \mathrm{C} 10 \mathrm{C} 14)\end{array}$ & ${ }^{3} \tau_{3 / R 6}$ \\
\hline 68 & $\beta(\mathrm{C} 7 \mathrm{~N} 3 \mathrm{C} 18)-\beta(\mathrm{C} 16 \mathrm{~N} 3 \mathrm{C} 18)$ & $\rho_{\mathrm{C} 18 \mathrm{~N} 3}$ \\
\hline 69 & $\gamma(\mathrm{N} 3 \mathrm{C} 7 \mathrm{C} 16 \mathrm{C} 18)$ & $\omega_{\mathrm{C} 18 \mathrm{~N} 3}$ \\
\hline 70 & $\beta(\mathrm{C} 10 \mathrm{H} 28 \mathrm{C} 14)-\beta(\mathrm{C} 15 \mathrm{H} 28 \mathrm{C} 14)$ & $\rho_{\mathrm{C} 14 \mathrm{H} 28}$ \\
\hline 71 & $\gamma(\mathrm{H} 28 \mathrm{C} 10 \mathrm{C} 15 \mathrm{C} 14)$ & $\omega_{\mathrm{C} 14 \mathrm{H} 28}$ \\
\hline 72 & $\beta(\mathrm{C} 14 \mathrm{H} 29 \mathrm{C} 15)-\beta(\mathrm{C} 13 \mathrm{H} 29 \mathrm{C} 15)$ & $\rho_{\mathrm{C} 15 \mathrm{H} 29}$ \\
\hline 73 & $\gamma(\mathrm{H} 29 \mathrm{C} 14 \mathrm{C} 13 \mathrm{C} 15)$ & $\omega_{\mathrm{C} 15 \mathrm{H} 29}$ \\
\hline 74 & $\beta(\mathrm{C} 15 \mathrm{H} 27 \mathrm{C} 13)-\beta(\mathrm{C} 12 \mathrm{H} 27 \mathrm{C} 13)$ & $\rho_{\mathrm{C} 13 \mathrm{H} 27}$ \\
\hline 75 & $\gamma(\mathrm{H} 27 \mathrm{C} 15 \mathrm{C} 12 \mathrm{C} 13)$ & $\omega_{\mathrm{C} 13 \mathrm{H} 27}$ \\
\hline 76 & $\beta(\mathrm{C} 13 \mathrm{H} 26 \mathrm{C} 12)-\beta(\mathrm{C} 11 \mathrm{H} 26 \mathrm{C} 12)$ & $\rho_{\mathrm{C} 12 \mathrm{H} 26}$ \\
\hline 77 & $\gamma(\mathrm{H} 26 \mathrm{C} 13 \mathrm{C} 11 \mathrm{C} 12)$ & $\omega_{\mathrm{C} 12 \mathrm{H} 26}$ \\
\hline 78 & $\beta(\mathrm{C} 8 \mathrm{H} 30 \mathrm{C} 19)-\beta(\mathrm{C} 6 \mathrm{H} 30 \mathrm{C} 19)$ & $\rho_{\mathrm{C} 19 \mathrm{H} 30}$ \\
\hline 79 & $\gamma(\mathrm{H} 30 \mathrm{C} 8 \mathrm{C} 6 \mathrm{C} 19)$ & $\omega_{\mathrm{C} 19 \mathrm{H} 30}$ \\
\hline 80 & $\beta(\mathrm{C} 19 \mathrm{H} 24 \mathrm{C} 6)-\beta(\mathrm{C} 7 \mathrm{H} 24 \mathrm{C} 6)$ & $\rho_{\mathrm{C} 6 \mathrm{H} 24}$ \\
\hline 81 & $\gamma(\mathrm{H} 24 \mathrm{C} 19 \mathrm{C} 7 \mathrm{C} 6)$ & $\omega_{\mathrm{C} 6 \mathrm{H} 24}$ \\
\hline 82 & $\beta(\mathrm{C} 6 \mathrm{H} 25 \mathrm{C} 7)-\beta(\mathrm{C} 18 \mathrm{H} 25 \mathrm{C} 7)$ & $\rho_{\mathrm{C} 7 \mathrm{H} 25}$ \\
\hline 83 & $\gamma(\mathrm{H} 25 \mathrm{C} 6 \mathrm{C} 18 \mathrm{C} 7)$ & $\omega_{\mathrm{C} 7 \mathrm{H} 25}$ \\
\hline 84 & $\beta(\mathrm{C} 16 \mathrm{O} 5 \mathrm{C} 9)-\beta(\mathrm{C} 10 \mathrm{O} 5 \mathrm{C} 9)$ & $\rho_{\mathrm{C} 9 \mathrm{O} 5}$ \\
\hline
\end{tabular}




\begin{tabular}{|c|c|c|}
\hline 85 & $\gamma(\mathrm{O} 5 \mathrm{C} 16 \mathrm{C} 10 \mathrm{C} 9)$ & $\omega_{\mathrm{C} 9 \mathrm{O} 5}$ \\
\hline 86 & $\beta(\mathrm{C} 8 \mathrm{O} 4 \mathrm{C} 17)-\beta(\mathrm{C} 11 \mathrm{O} 4 \mathrm{C} 17)$ & $\rho_{\mathrm{C} 17 \mathrm{O} 4}$ \\
\hline 87 & $\gamma(\mathrm{O} 4 \mathrm{C} 8 \mathrm{C} 11 \mathrm{C} 17)$ & $\omega_{\mathrm{C} 17 \mathrm{O} 4}$ \\
\hline 88 & $\tau(\mathrm{C} 2 \mathrm{~N} 3 \mathrm{C} 18 \mathrm{C} 7)+\tau(\mathrm{H} 23 \mathrm{~N} 3 \mathrm{C} 18 \mathrm{C} 7)+\tau(\mathrm{C} 2 \mathrm{~N} 3 \mathrm{C} 18 \mathrm{C} 16)+\tau(\mathrm{H} 23 \mathrm{~N} 3 \mathrm{C} 18 \mathrm{C} 16)$ & $\tau_{\mathrm{N} 3 \mathrm{C} 18}$ \\
\hline 89 & $\begin{array}{l}\tau(\mathrm{C} 20 \mathrm{C} 2 \mathrm{~N} 3 \mathrm{C} 18)+\tau(\mathrm{H} 21 \mathrm{C} 2 \mathrm{~N} 3 \mathrm{C} 18)+\tau(\mathrm{H} 22 \mathrm{C} 2 \mathrm{~N} 3 \mathrm{C} 18)+\tau(\mathrm{C} 20 \mathrm{C} 2 \mathrm{~N} 3 \mathrm{H} 23) \\
+\tau(\mathrm{H} 21 \mathrm{C} 2 \mathrm{~N} 3 \mathrm{H} 23)+\tau(\mathrm{H} 22 \mathrm{C} 2 \mathrm{~N} 3 \mathrm{H} 23)\end{array}$ & $\tau_{\mathrm{C} 2 \mathrm{~N} 3}$ \\
\hline 90 & $\begin{array}{l}\tau(\mathrm{N} 3 \mathrm{C} 2 \mathrm{C} 20 \mathrm{~S} 1)+\tau(\mathrm{H} 21 \mathrm{C} 2 \mathrm{C} 20 \mathrm{~S} 1)+\tau(\mathrm{H} 22 \mathrm{C} 2 \mathrm{C} 20 \mathrm{~S} 1)+\tau(\mathrm{N} 3 \mathrm{C} 2 \mathrm{C} 20 \mathrm{H} 31) \\
+\tau(\mathrm{H} 21 \mathrm{C} 2 \mathrm{C} 20 \mathrm{H} 31)+\tau(\mathrm{H} 22 \mathrm{C} 2 \mathrm{C} 20 \mathrm{H} 31)+\tau(\mathrm{N} 3 \mathrm{C} 2 \mathrm{C} 20 \mathrm{H} 32)+\tau(\mathrm{H} 21 \mathrm{C} 2 \mathrm{C} 20 \mathrm{H} 32) \\
+\tau(\mathrm{H} 22 \mathrm{C} 2 \mathrm{C} 20 \mathrm{H} 32)\end{array}$ & $\tau_{\mathrm{C} 2 \mathrm{C} 20}$ \\
\hline 91 & $\tau(\mathrm{H} 33 \mathrm{~S} 1 \mathrm{C} 20 \mathrm{C} 2)+\tau(\mathrm{H} 33 \mathrm{~S} 1 \mathrm{C} 20 \mathrm{H} 31)+\tau(\mathrm{H} 33 \mathrm{~S} 1 \mathrm{C} 20 \mathrm{H} 32)$ & $\tau_{\mathrm{S} 1 \mathrm{C} 20}$ \\
\hline 92 & $\tau(\mathrm{C} 9 \mathrm{C} 16 \mathrm{C} 8 \mathrm{C} 19)-\tau(\mathrm{C} 18 \mathrm{C} 16 \mathrm{C} 8 \mathrm{C} 17)$ & ${ }^{2} b f l_{R R}$ \\
\hline 93 & $\tau(\mathrm{C} 14 \mathrm{C} 10 \mathrm{C} 11 \mathrm{C} 17)-\tau(\mathrm{C} 9 \mathrm{C} 10 \mathrm{C} 11 \mathrm{C} 12)$ & ${ }^{4} b f l_{R R}$ \\
\hline
\end{tabular}


Table S.4. Frequencies of normal vibrations, IR and Raman intensities and PEDs for ScTT conformer calculated at the B3LYP/6-311++G(d,p) level of theory.

\begin{tabular}{|c|c|c|c|c|}
\hline $\mathbf{N r}$ & $\begin{array}{l}\text { Freq. } \\
{\left[\mathrm{cm}^{-1}\right]}\end{array}$ & IR Int. & \begin{tabular}{|c|} 
Raman \\
Int.
\end{tabular} & PED (contributions $>5 \%)$ \\
\hline 1 & 23.0 & 0.3 & 8.1 & $\tau_{\mathrm{N} 3 \mathrm{C} 18}(41), \omega_{\mathrm{N} 3 \mathrm{H} 23}(32),{ }^{2} \tau_{3 / R 6}(8),{ }^{2} \tau_{2 / R 6}(6)$ \\
\hline 2 & 35.4 & 0.7 & 2.7 & $\tau_{\mathrm{C}_{2} \mathrm{C} 20}(30), \tau_{\mathrm{C}_{2} \mathrm{~N} 3}(21),{ }^{2} \tau_{3 / R}(11),{ }^{\mathrm{I}}{ }{ }_{3 / R 6}(8), \tau_{\mathrm{N} 3 \mathrm{C} 18}(8), \beta_{\mathrm{C} 18 \mathrm{~N} 3 \mathrm{C} 2}(7)$ \\
\hline 3 & 43.3 & 4.3 & 0.5 & ${ }^{2} \tau_{3 / R \sigma}(43),{ }^{2} \tau_{2 / R \sigma}(11), \tau_{\mathrm{C}_{2} \mathrm{C} 20}(10),{ }^{1} \tau_{3 / R \sigma}(10), \omega_{\mathrm{N} 3 \mathrm{H} 23}(5)$ \\
\hline 4 & 74.9 & 1.1 & 0.3 & ${ }^{2} \tau_{2 / R 6}(30), \omega_{\mathrm{N} 3 \mathrm{H} 23}(25),{ }^{1} \tau_{3 / R 6}(14), \tau_{\mathrm{C} 2 \mathrm{~N} 3}(7)$ \\
\hline 5 & 101.3 & 7.0 & 0.4 & $\tau_{\mathrm{C} 2 \mathrm{~N} 3}(39), \tau_{\mathrm{C} 2 \mathrm{C} 20}(18), \tau_{\mathrm{S} 1 \mathrm{C} 20}(13),{ }^{1} \tau_{2 / R 6}(8)$ \\
\hline 6 & 119.0 & 0.2 & 0.8 & ${ }^{2} \tau_{1 / R \sigma}(44),{ }^{3} \tau_{2 / R \sigma}(17),{ }^{2} \tau_{2 / R 6}(10),{ }^{3} \tau_{3 / R 6}(9)$ \\
\hline 7 & 151.3 & 11.8 & 1.0 & $\tau_{\mathrm{S} 1 \mathrm{C} 20}(80), \rho_{\mathrm{C} 20 \mathrm{H} 31 \mathrm{H} 32}(5)$ \\
\hline 8 & 158.2 & 1.1 & 0.2 & $\delta_{2 / \mathrm{C} 20 \mathrm{H} 31 \mathrm{H} 32}(20), \rho_{\mathrm{C} 18 \mathrm{~N} 3}(17), \tau_{\mathrm{C}_{2 N} 3}(8), v_{\mathrm{C} 9 \mathrm{C} 10}(5)$ \\
\hline 9 & 167.3 & 0.2 & 0.0 & ${ }^{4} b f l_{R R}(27),{ }^{2} b f l_{R R}(21), \tau_{N 3 C l 8}(9),{ }^{2} \tau_{3 / R 6}(7), \omega_{C 17 O 4}(6), \omega_{C 9 O 5}(6)$ \\
\hline 10 & 195.5 & 0.4 & 0.9 & ${ }^{1} \tau_{2 / R 6}(26),{ }^{1} \tau_{3 / R 6}(24),{ }^{4} b f l_{R R}(9), \omega_{C 19 H 30}(6),{ }^{2} \tau_{1 / R 6}(5)$ \\
\hline 11 & 225.2 & 3.7 & 0.4 & 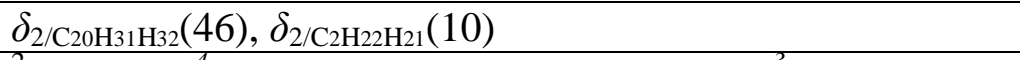 \\
\hline 12 & 239.2 & 0.7 & 0.9 & ${ }^{2} b f l_{R R}(27),{ }^{4} b f l_{R R}(12), \delta_{2 / C_{2} H_{22} H_{21}}(9), \omega_{C 18 N 3}(7),{ }^{3} \tau_{3 / R 6}(6)$ \\
\hline 13 & 284.0 & 3.8 & 0.5 & $\rho_{\mathrm{C} 18 \mathrm{~N} 3}(18), \beta_{\mathrm{C} 18 \mathrm{~N} 3 \mathrm{C} 2_{2}}(11), \delta_{2 / \mathrm{C} 20 \mathrm{H} 31 \mathrm{H} 32}(10),{ }^{2} \beta_{2 / R 6}(10)$ \\
\hline 14 & 305.6 & 2.8 & 0.4 & $\begin{array}{l}\delta_{2 / \mathrm{C}_{2} \mathrm{H}_{22} \mathrm{H}_{21}}(15), \omega_{\mathrm{N}_{3} \mathrm{H}_{23}}(11), \beta_{\mathrm{C} 18 \mathrm{~N} 3 \mathrm{C} 2}(11),{ }^{2} b f l_{R R}(7),{ }^{I} \tau_{3 / R 6}(6), \\
\delta_{2 / \mathrm{C}_{20} \mathrm{H} 31 \mathrm{H} 32}(6)\end{array}$ \\
\hline 15 & 345.0 & 1.6 & 0.1 & ${ }^{2} \beta_{3 / R 6}(26), \rho_{\mathrm{C} 17 \mathrm{O} 4}(20),{ }^{2} \beta_{2 / R 6}(14), \rho_{\mathrm{C} 18 \mathrm{~N} 3}(13)$ \\
\hline 16 & 366.1 & 7.0 & 0.7 & $\begin{array}{l}{ }^{2} \beta_{3 / R \sigma}(18),{ }^{2} \beta_{2 / R 6}(10), v_{\mathrm{C} 11 \mathrm{C} 17}(9),{ }^{1} \beta_{3 / R 6}(7), v_{\mathrm{C} 9 \mathrm{C} 16}(7), v_{\mathrm{C} 9 \mathrm{C} 10}(6), \\
\rho_{\mathrm{C} 17 \mathrm{O} 4}(6)\end{array}$ \\
\hline 17 & 411.3 & 1.2 & 0.3 & $\begin{array}{l}{ }^{2} \beta_{2 / R \sigma}(22), \delta_{2 / \mathrm{C}_{2} \mathrm{H} 22 \mathrm{H} 21}(10),{ }^{3} \beta_{2 / R \sigma}(7),{ }^{1} \beta_{2 / R \sigma}(6),{ }^{2} \beta_{3 / R \sigma}(6), \\
v_{\mathrm{S}_{1} \mathrm{C} 20}(6),{ }^{1} \beta_{3 / R 6}(5)\end{array}$ \\
\hline 18 & 427.2 & 11.6 & 0.3 & ${ }^{3} \tau_{3 / R 6}(43), \rho_{\mathrm{C} 9 \mathrm{O} 5}(11),{ }^{4} b f l_{R R}(11)$ \\
\hline 19 & 429.3 & 12.5 & 0.4 & ${ }^{3} \tau_{3 / R 6}(39), \rho_{\mathrm{C} 9 \mathrm{O} 5}(11),{ }^{4} b f l_{R R}(9), \rho_{\mathrm{Cl}_{17 O 4}}(6)$ \\
\hline 20 & 432.9 & 0.3 & 0.0 & ${ }^{3} \tau_{2 / R 6}(32),{ }^{1} \tau_{2 / R 6}(20),{ }^{1} \tau_{3 / R 6}(16), \omega_{\mathrm{C} 9 \mathrm{O} 5}(6),{ }^{2} \tau_{1 / R 6}(6)$ \\
\hline 21 & 477.4 & 1.8 & 10.3 & ${ }^{2} \beta_{3 / R \sigma}(31),{ }^{2} \beta_{2 / R 6}(16),{ }^{1} \beta_{3 / R \sigma}(11),{ }^{3} \beta_{3 / R 6}(7), v_{\mathrm{C} 10 \mathrm{C} 11}(6)$ \\
\hline 22 & 490.0 & 1.8 & 0.0 & ${ }^{3} \tau_{2 / R \sigma}(23),{ }^{1} \tau_{3 / R \sigma}(22),{ }^{1} \tau_{2 / R \sigma}(11),{ }^{2} \tau_{2 / R \sigma}(9),{ }^{1} \tau_{1 / R \sigma}(6),{ }^{3} \tau_{3 / R \sigma}(5)$ \\
\hline 23 & 509.8 & 5.3 & 1.3 & $\begin{array}{l}{ }^{1} \beta_{2 / R \sigma}(16),{ }^{2} \beta_{2 / R 6}(10), v_{\mathrm{S} 1 \mathrm{C} 20}(9), \rho_{\mathrm{C} 18 \mathrm{~N} 3}(6), \delta_{2 / \mathrm{C} 2 \mathrm{H} 22 \mathrm{H} 21}(6), \\
{ }^{1} \beta_{3 / R 6}(6),{ }^{3} \beta_{2 / R 6}(6)\end{array}$ \\
\hline 24 & 557.8 & 5.2 & 0.1 & $\omega_{\mathrm{C} 18 \mathrm{~N} 3}(29),{ }^{1} \tau_{3 / R \sigma}(13),{ }^{1} \tau_{2 / R \sigma}(13),{ }^{1} \tau_{1 / R 6}(10),{ }^{2} b f l_{R R}(6)$ \\
\hline 25 & 602.5 & 5.0 & 0.3 & $\begin{array}{l}{ }^{3} \beta_{2 / R 6}(14),{ }^{1} \beta_{3 / R 6}(13), \beta_{\mathrm{C} 18 \mathrm{~N}_{3} \mathrm{C} 2}(12),{ }^{1} \beta_{2 / R 6}(11), \rho_{\mathrm{C} 18 \mathrm{~N} 3}(8), \\
{ }^{2} \beta_{\text {trig/R6 }}(7),{ }^{3} \beta_{3 / R 6}(5)\end{array}$ \\
\hline 26 & 634.0 & 0.9 & 0.0 & ${ }^{2} \beta_{\text {trig/R6 }}(39),{ }^{1} \beta_{2 / R 6}(16), \rho_{\mathrm{C} 18 \mathrm{~N} 3}(8), \beta_{\mathrm{C} 18 \mathrm{~N} 3 \mathrm{C} 2_{2}}(8)$ \\
\hline 27 & 669.1 & 2.6 & 0.1 & ${ }^{3} \tau_{1 / R 6}(37), \omega_{\mathrm{C} 9 \mathrm{O} 5}(13),{ }^{1} \tau_{1 / R 6}(12), \omega_{\mathrm{C} 18 \mathrm{~N} 3}(11)$ \\
\hline 28 & 673.5 & 13.4 & 1.7 & ${ }^{3} \beta_{3 / R 6}(41),{ }^{3} \beta_{2 / R 6}(10), v_{\mathrm{C} 10 \mathrm{C} 11}(7)$ \\
\hline 29 & 687.1 & 58.5 & 0.1 & $\tau_{\mathrm{N} 3 \mathrm{C} 18}(28), \omega_{\mathrm{N} 3 \mathrm{H} 23}(19), \tau_{\mathrm{C} 2 \mathrm{~N} 3}(13),{ }^{1} \tau_{1 / R 6}(12)$ \\
\hline 30 & 705.3 & 4.3 & 1.4 & $\rho_{\mathrm{C} 9 \mathrm{O} 5}(20), \rho_{\mathrm{C} 17 \mathrm{O} 4}(17),{ }^{3} \beta_{2 / R 6}(17),{ }^{1} \beta_{2 / R 6}(10),{ }^{1} \beta_{3 / R 6}(9),{ }^{3} \beta_{3 / R 6}(5)$ \\
\hline 31 & 725.8 & 32.8 & 0.0 & ${ }^{3} \tau_{1 / R 6}(27),{ }^{1} \tau_{1 / R 6}(26), \omega_{\mathrm{C} 6 \mathrm{H} 24}(8), \omega_{\mathrm{C} 15 \mathrm{H} 29}(8), \omega_{\mathrm{C} 17 \mathrm{O} 4}(7)$ \\
\hline 32 & 747.4 & 63.4 & 0.1 & $\begin{array}{l}{ }^{1} \tau_{1 / R 6}(15), \omega_{\mathrm{C} 18 \mathrm{~N} 3}(14), \omega_{\mathrm{C} 13 \mathrm{H} 27}(13), \omega_{\mathrm{C} 9 \mathrm{O} 5}(8),{ }^{3} \tau_{1 / R 6}(8), \\
\omega_{\mathrm{C} 17 \mathrm{O} 4}(7), \omega_{\mathrm{C} 14 \mathrm{H} 28}(7), \omega_{\mathrm{C} 15 \mathrm{H} 29}(6)\end{array}$ \\
\hline 33 & 750.8 & 2.9 & 5.6 & $v_{\mathrm{S} 1 \mathrm{C} 20_{0}}(61), \delta_{2 / \mathrm{C} 2 \mathrm{H} 22 \mathrm{H} 21}(17), \delta_{2 / \mathrm{C} 20 \mathrm{H} 31 \mathrm{H} 32}(8)$ \\
\hline 34 & 782.7 & 1.2 & 1.4 & $\rho_{\mathrm{C} 20 \mathrm{H} 31 \mathrm{H} 32}(25), \rho_{\mathrm{C} 2 \mathrm{H} 22 \mathrm{H} 21}(19),{ }^{1} \beta_{3 / R 6}(10),{ }^{3} \beta_{2 / R 6}(7),{ }^{1} \beta_{\text {trig/R6}}(7)$ \\
\hline 35 & 788.1 & 4.9 & 0.0 & $\begin{array}{l}{ }^{3} \tau_{1 / R 6}(18),{ }^{2} \tau_{1 / R 6}(13), \omega_{\mathrm{C} 7 \mathrm{H} 25}(13), \omega_{\mathrm{C} 19 \mathrm{H} 30}(11), \omega_{\mathrm{C} 6 \mathrm{H} 24}(11), \\
\omega_{\mathrm{C} 17 \mathrm{O} 4}(10), \omega_{\mathrm{C} 9 \mathrm{O} 5}(9), \omega_{\mathrm{C} 15 \mathrm{H} 29}(5)\end{array}$ \\
\hline 36 & 799.5 & 2.7 & 0.4 & $\begin{array}{l}\rho_{\mathrm{C}_{20 \mathrm{H} 31 \mathrm{H} 32}}(22),{ }^{1} \beta_{\text {trig/R6}}(11), \rho_{\mathrm{C}_{2} \mathrm{H} 22 \mathrm{H} 21}(11),{ }^{1} \beta_{3 / R 6}(7),{ }^{3} \beta_{2 / R 6}(6), \\
{ }^{3} \beta_{\text {trig/R6 }}(5)\end{array}$ \\
\hline
\end{tabular}




\begin{tabular}{|c|c|c|c|c|}
\hline 37 & 809.8 & 1.0 & 0.1 & $\begin{array}{l}\omega_{\mathrm{C} 9 \mathrm{O} 5}(27), \omega_{\mathrm{C} 13 \mathrm{H} 27}(14),{ }^{1} \tau_{1 / R 6}(11), \omega_{\mathrm{C} 18 \mathrm{~N} 3}(8),{ }^{2} \tau_{1 / R 6}(8), \\
\omega_{\mathrm{C} 15 \mathrm{H} 29}(7)\end{array}$ \\
\hline 38 & 827.6 & 26.2 & 0.0 & $\begin{array}{l}\omega_{\mathrm{C} 7 \mathrm{H} 25}(23), \omega_{\mathrm{C} 17 \mathrm{O} 4}(17),{ }^{1} \tau_{1 / R 6}(16), \omega_{\mathrm{C} 18 \mathrm{~N} 3}(9),{ }^{2} \tau_{1 / R 6}(7), \\
\omega_{\mathrm{C} 6 \mathrm{H} 24}(7), \omega_{\mathrm{C} 15 \mathrm{H} 29}(6)\end{array}$ \\
\hline 39 & 844.1 & 2.2 & 1.2 & $\beta_{\mathrm{C}_{20 \mathrm{~S} 1 \mathrm{H} 33}}(53), v_{\mathrm{C}_{2} \mathrm{C} 20}(21), v_{\mathrm{S} 1 \mathrm{C} 20}(6)$ \\
\hline 40 & 877.9 & 19.6 & 2.5 & 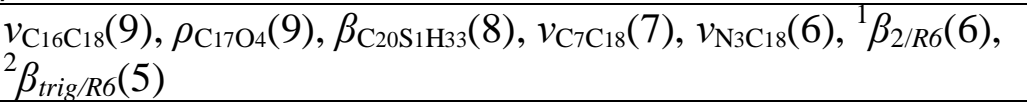 \\
\hline 41 & 912.4 & 0.4 & 0.0 & $\begin{array}{l}\omega_{\mathrm{C} 19 \mathrm{H} 30}(23), \omega_{\mathrm{C} 12 \mathrm{H} 26}(17), \omega_{\mathrm{C} 7 \mathrm{H} 25}(17), \omega_{\mathrm{C} 14 \mathrm{H} 28}(17), \omega_{\mathrm{C} 15 \mathrm{H} 29}(8), \\
\omega_{\mathrm{C} 13 \mathrm{H} 27}(6)\end{array}$ \\
\hline 42 & 919.0 & 0.7 & 0.0 & $\omega_{\mathrm{C} 19 \mathrm{H} 30}(27), \omega_{\mathrm{C} 7 \mathrm{H} 25}(18), \omega_{\mathrm{C} 14 \mathrm{H} 28}(13), \omega_{\mathrm{C} 12 \mathrm{H} 26}(12), \omega_{\mathrm{C} 13 \mathrm{H} 27}(7)$ \\
\hline 43 & 928.8 & 2.1 & 1.3 & ${ }^{1} \beta_{\text {trig/R6 }}(25),{ }^{3} \beta_{\text {trig/R6 }}(24), \rho_{\mathrm{C} 9 \mathrm{O} 5}(14), v_{\mathrm{C} 16 \mathrm{C} 18}(7), \rho_{\mathrm{C} 17 \mathrm{O} 4}(5)$ \\
\hline 44 & 999.8 & 71.9 & 1.2 & $v_{\mathrm{C} 2 \mathrm{~N} 3}(11), v_{\mathrm{C} 8 \mathrm{C} 17}(10), \omega_{\mathrm{C} 6 \mathrm{H} 24}(7), v_{\mathrm{C} 11 \mathrm{C} 12}(7), \rho_{\mathrm{C} 17 \mathrm{O} 4}(6), v_{\mathrm{C} 8 \mathrm{C} 19}(5)$ \\
\hline 45 & 1000.5 & 8.8 & 0.2 & $\omega_{\mathrm{C} 6 \mathrm{H} 24}(43), \omega_{\mathrm{C} 19 \mathrm{H} 30}(18), \omega_{\mathrm{C} 7 \mathrm{H} 25}(9),{ }^{1} \tau_{1 / R 6}(6)$ \\
\hline 46 & 1002.8 & 2.2 & 0.0 & $\omega_{\mathrm{C} 12 \mathrm{H} 26}(30), \omega_{\mathrm{C} 14 \mathrm{H} 28}(28), \omega_{\mathrm{C} 13 \mathrm{H} 27}(10), \omega_{\mathrm{C} 15 \mathrm{H} 29}(9), \omega_{\mathrm{C} 6 \mathrm{H} 24}(8)$ \\
\hline 47 & 1016.7 & 0.0 & 0.0 & $\omega_{\mathrm{C} 15 \mathrm{H} 29}(30), \omega_{\mathrm{C} 13 \mathrm{H} 27}(28), \omega_{\mathrm{C} 14 \mathrm{H} 28}(15), \omega_{\mathrm{C} 12 \mathrm{H} 26}(14),{ }^{3} \tau_{1 / R 6}(12)$ \\
\hline 48 & 1026.4 & 22.1 & 0.8 & $\tau_{\mathrm{C} 20 \mathrm{H} 31 \mathrm{H} 32}(29), \rho_{\mathrm{C}_{2} \mathrm{H} 22 \mathrm{H} 21}(27), \rho_{\mathrm{C} 20 \mathrm{H} 31 \mathrm{H} 32}(22)$ \\
\hline 49 & 1057.4 & 0.3 & 5.0 & $v_{\mathrm{C} 13 \mathrm{C} 15}(34), v_{\mathrm{C} 14 \mathrm{C} 15}(13), \rho_{\mathrm{C} 12 \mathrm{H} 26}(12), \rho_{\mathrm{C} 14 \mathrm{H} 28}(12), v_{\mathrm{C} 12 \mathrm{C} 13}(11)$ \\
\hline 50 & 1062.8 & 7.0 & 0.9 & $v_{\mathrm{C}_{2} \mathrm{C} 20}(62), \beta_{\mathrm{C}_{20} \mathrm{~S}_{1} \mathrm{H}_{33}}(22), \delta_{2 / \mathrm{C} 2 \mathrm{H} 22 \mathrm{H} 21}(7), \delta_{2 / \mathrm{C}_{20} \mathrm{H}_{31} \mathrm{H}_{32}}(5)$ \\
\hline 51 & 1097.5 & 29.5 & 1.6 & $v_{\mathrm{C} 6 \mathrm{C} 19}(31), \rho_{\mathrm{C} 19 \mathrm{H} 30}(16),{ }^{1} \beta_{\text {trig/R6 }}(14), v_{\mathrm{C} 6 \mathrm{C} 7}(8), v_{\mathrm{C}_{2} \mathrm{~N}_{3}}(5), v_{\mathrm{C} 9 \mathrm{C} 16}(5)$ \\
\hline 52 & 1113.0 & 1.1 & 0.4 & $\begin{array}{l}{ }^{3} \beta_{\text {trig/R6 }}(37), v_{\mathrm{C} 12 \mathrm{C} 13}(9), v_{\mathrm{C} 14 \mathrm{C} 15}(8), \rho_{\mathrm{C} 13 \mathrm{H} 27}(8), \rho_{\mathrm{C} 15 \mathrm{H} 29}(7), \\
v_{\mathrm{C} 9 \mathrm{C} 10}(6), \rho_{\mathrm{C} 14 \mathrm{H} 28}(5), v_{\mathrm{C} 11 \mathrm{C} 17}(5)\end{array}$ \\
\hline 53 & 1130.0 & 3.6 & 2.0 & $v_{\mathrm{C} 2 \mathrm{~N} 3}(42), \rho_{\mathrm{C} 18 \mathrm{~N} 3}(8), \tau_{\mathrm{C} 20 \mathrm{H} 31 \mathrm{H} 32}(7), v_{\mathrm{C} 8 \mathrm{C} 17}(6)$ \\
\hline 54 & 1175.6 & 10.2 & 3.9 & $\begin{array}{l}\rho_{\mathrm{C} 13 \mathrm{H} 27}(15), \rho_{\mathrm{C} 12 \mathrm{H} 26}(11), v_{\mathrm{C} 9 \mathrm{C} 16}(10),{ }^{1} \beta_{\text {trig/R6 }}(8), v_{\mathrm{C} 9 \mathrm{C} 10}(7), \\
{ }^{3} \beta_{\text {trig/R6 }}(7), v_{\mathrm{C} 7 \mathrm{C} 18}(6)\end{array}$ \\
\hline 55 & 1182.0 & 19.3 & 0.4 & $\rho_{\mathrm{C} 15 \mathrm{H} 29}(29), \rho_{\mathrm{C} 14 \mathrm{H} 28}(12), \rho_{\mathrm{C} 13 \mathrm{H} 27}(9), v_{\mathrm{C} 14 \mathrm{C} 15}(7), v_{\mathrm{C} 13 \mathrm{C} 15}(6)$ \\
\hline 56 & 1196.6 & 16.9 & 10.7 & $\begin{array}{l}\rho_{\mathrm{C} 6 \mathrm{H} 24}(20), \rho_{\mathrm{C} 13 \mathrm{H} 27}(7), \rho_{\mathrm{C} 7 \mathrm{H} 25}(7), \tau_{\mathrm{C} 20 \mathrm{H} 31 \mathrm{H} 32}(6), v_{\mathrm{C} 16 \mathrm{C} 18}(5), \\
\rho_{\mathrm{C} 15 \mathrm{H} 29}(5)\end{array}$ \\
\hline 57 & 1210.3 & 37.6 & 1.3 & $\begin{array}{l}\rho_{\mathrm{C} 7 \mathrm{H} 25}(20), \tau_{\mathrm{C} 20 \mathrm{H} 31 \mathrm{H} 32}(19), \rho_{\mathrm{C} 6 \mathrm{H} 24}(13), \rho_{\mathrm{C} 2 \mathrm{H} 22 \mathrm{H} 21}(9), \rho_{\mathrm{C} 19 \mathrm{H} 30}(7), \\
v_{\mathrm{C} 6 \mathrm{C} 7}(6), \tau_{\mathrm{C} 2 \mathrm{H} 22 \mathrm{H} 21}(5)\end{array}$ \\
\hline 58 & 1217.8 & 19.3 & 2.6 & $\begin{array}{l}\rho_{\mathrm{C} 19 \mathrm{H} 30}(19), \tau_{\mathrm{C} 20 \mathrm{H} 31 \mathrm{H} 32}(8), v_{\mathrm{C} 11 \mathrm{C} 17}(8), \rho_{\mathrm{C} 14 \mathrm{H} 28}(7),{ }^{3} \beta_{\text {trig/R6 }}(6), \\
v_{\mathrm{C} 8 \mathrm{C} 19}(6)\end{array}$ \\
\hline 59 & 1264.0 & 49.6 & 3.0 & $\rho_{\mathrm{C} 12 \mathrm{H} 26}(16),{ }^{2} \beta_{\text {trig/R6 }}(9), \rho_{\mathrm{C} 14 \mathrm{H} 28}(9), v_{\mathrm{C} 11 \mathrm{C} 12}(7), v_{\mathrm{C} 7 \mathrm{C} 18}(6)$ \\
\hline 60 & 1274.9 & 98.6 & 0.2 & $\omega_{\mathrm{C}_{20} \mathrm{H} 31 \mathrm{H} 32}(78), \omega_{\mathrm{C}_{2} \mathrm{H} 22 \mathrm{H} 21}(10)$ \\
\hline 61 & 1289.4 & 571.5 & 8.3 & $\begin{array}{l}v_{\mathrm{C} 9 \mathrm{C} 10}(13), v_{\mathrm{C} 8 \mathrm{C} 16}(8), \rho_{\mathrm{C} 14 \mathrm{H} 28}(8), v_{\mathrm{C} 9 \mathrm{C} 16}(7), v_{\mathrm{C} 7 \mathrm{C} 18}(6), \rho_{\mathrm{C} 19 \mathrm{H} 30}(6), \\
v_{\mathrm{C} 8 \mathrm{C} 17}(6), v_{\mathrm{C} 6 \mathrm{C} 19}(5), v_{\mathrm{C} 16 \mathrm{C} 18}(5)\end{array}$ \\
\hline 62 & 1316.1 & 135.5 & 1.5 & $v_{\mathrm{C} 11 \mathrm{C} 17_{17}}(19), v_{\mathrm{C} 8 \mathrm{C} 17}(13), \rho_{\mathrm{C} 12 \mathrm{H} 26}(9), \rho_{\mathrm{C} 17 \mathrm{O} 4}(8),{ }^{2} \beta_{2 / R 6}(6)$ \\
\hline 63 & 1328.8 & 74.4 & 7.6 & $\tau_{\mathrm{C} 2 \mathrm{H} 22 \mathrm{H} 21}(29), v_{\mathrm{N} 3 \mathrm{C} 18}(18),{ }^{1} \beta_{\text {trig/R6 }}(8), v_{\mathrm{C} 16 \mathrm{C} 18}(8), \rho_{\mathrm{N} 3 \mathrm{H} 23}(6)$ \\
\hline 64 & 1339.6 & 113.1 & 2.1 & $\begin{array}{l}\tau_{\mathrm{C}_{2} \mathrm{H} 22 \mathrm{H} 21}(23), \rho_{\mathrm{N}_{3} \mathrm{H} 23}(11), v_{\mathrm{N} 3 \mathrm{C} 18}(11), v_{\mathrm{C} 16 \mathrm{C} 18}(8), v_{\mathrm{C} 9 \mathrm{C} 16}(8), \\
\rho_{\mathrm{C} 6 \mathrm{H} 24}(6)\end{array}$ \\
\hline 65 & 1358.6 & 17.2 & 0.9 & $\begin{array}{l}v_{\mathrm{C} 10 \mathrm{C} 14}(15), v_{\mathrm{C} 10 \mathrm{C} 11}(15), v_{\mathrm{C} 11 \mathrm{C} 12}(14), v_{\mathrm{C} 13 \mathrm{C} 15}(14), v_{\mathrm{C} 12 \mathrm{C} 13}(13), \\
v_{\mathrm{C} 14 \mathrm{C} 15}(13)\end{array}$ \\
\hline 66 & 1379.6 & 16.4 & 1.7 & $\begin{array}{l}v_{\mathrm{C} 16 \mathrm{C} 18}(17), v_{\mathrm{C} 8 \mathrm{C} 19}(11), v_{\mathrm{C} 8 \mathrm{C} 16}(11), v_{\mathrm{C} 9 \mathrm{C} 16}(8), v_{\mathrm{C} 6 \mathrm{C} 19}(7), v_{\mathrm{C} 6 \mathrm{C} 7}(6), \\
v_{\mathrm{C} 7 \mathrm{C} 18}(6), \rho_{\mathrm{C} 9 \mathrm{O} 5}(6), v_{\mathrm{C} 9 \mathrm{C} 10}(5)\end{array}$ \\
\hline 67 & 1397.5 & 17.2 & 1.0 & $\omega_{\mathrm{C} 2 \mathrm{H} 22 \mathrm{H} 21}(72), \omega_{\mathrm{C} 20 \mathrm{H} 31 \mathrm{H} 32}(7)$ \\
\hline 68 & 1435.5 & 31.6 & 0.4 & $\rho_{\mathrm{C} 7 \mathrm{H} 25}(25), v_{\mathrm{C} 6 \mathrm{C} 7}(11), v_{\mathrm{C} 8 \mathrm{C} 19}(11), v_{\mathrm{C} 8 \mathrm{C} 16}(6), v_{\mathrm{C} 9 \mathrm{C} 16}(6)$ \\
\hline 69 & 1478.5 & 0.3 & 4.3 & $\begin{array}{l}\rho_{\mathrm{C} 15 \mathrm{H} 29}(15), \rho_{\mathrm{C} 13 \mathrm{H} 27}(14), v_{\mathrm{C} 10 \mathrm{C} 14}(7), \rho_{\mathrm{C} 19 \mathrm{H} 30}(7), v_{\mathrm{C} 11 \mathrm{C} 12}(6), \\
v_{\mathrm{C} 8 \mathrm{C} 16}(6)\end{array}$ \\
\hline 70 & 1487.3 & 3.9 & 1.4 & $\begin{array}{l}\delta_{1 / \mathrm{C} 20 \mathrm{H} 31 \mathrm{H} 32}(13), \rho_{\mathrm{C} 19 \mathrm{H} 30}(8), \delta_{1 / \mathrm{C}_{2} \mathrm{H} 22 \mathrm{H} 21}(7), \rho_{\mathrm{C} 13 \mathrm{H} 27}(7), v_{\mathrm{C} 8 \mathrm{C} 16}(6), \\
\rho_{\mathrm{C} 6 \mathrm{H} 24}(6), \rho_{\mathrm{C} 15 \mathrm{H} 29}(6)\end{array}$ \\
\hline
\end{tabular}




\begin{tabular}{|c|c|c|c|c|}
\hline 71 & 1491.7 & 17.4 & 1.0 & $\delta_{1 / \mathrm{C}_{2} 0 \mathrm{H}_{31} \mathrm{H}_{32}}(72)$ \\
\hline 72 & 1507.5 & 4.1 & 2.1 & $\rho_{\mathrm{C} 14 \mathrm{H} 28}(20), \rho_{\mathrm{C} 12 \mathrm{H} 26}(19), v_{\mathrm{C} 10 \mathrm{C} 11}(17), v_{\mathrm{C} 13 \mathrm{C} 15}(8)$ \\
\hline 73 & 1514.4 & 4.9 & 0.5 & $\delta_{1 / \mathrm{C}_{2} \mathrm{H}_{22} \mathrm{H}_{21}}(77), \delta_{1 / \mathrm{C}_{20} \mathrm{H}_{31} \mathrm{H}_{32}}(6)$ \\
\hline 74 & 1553.5 & 198.3 & 1.7 & $v_{\mathrm{C} 6 \mathrm{C} 19}(19), \rho_{\mathrm{N} 3 \mathrm{H} 23}(18), v_{\mathrm{N} 3 \mathrm{C} 18}(16), \rho_{\mathrm{C} 6 \mathrm{H} 24}(12), v_{\mathrm{C} 6 \mathrm{C} 7}(8), v_{\mathrm{C} 8 \mathrm{C} 16}(6)$ \\
\hline 75 & 1611.0 & 33.3 & 10.8 & $\begin{array}{l}v_{\mathrm{C} 13 \mathrm{C} 15_{15}}(14), v_{\mathrm{C} 10 \mathrm{C} 11}(13), v_{\mathrm{O} 5 \mathrm{C} 9}(10), v_{\mathrm{C} 12 \mathrm{C} 13}(7),{ }^{3} \beta_{3 / R 6}(6), \\
v_{\mathrm{C}_{10 \mathrm{C} 14}}(5), v_{\mathrm{C} 8 \mathrm{C} 19}(5), \rho_{\mathrm{C} 13 \mathrm{H} 27}(5)\end{array}$ \\
\hline 76 & 1614.2 & 89.7 & 7.9 & $\begin{array}{l}\rho_{\mathrm{N}_{3} \mathrm{H} 23}(21), v_{\mathrm{C} 6 \mathrm{C} 19}(9), v_{\mathrm{C} 8 \mathrm{C} 19}(8), v_{\mathrm{C} 16 \mathrm{C} 18}(8), v_{\mathrm{C} 7 \mathrm{C} 18}(7),{ }^{1} \beta_{2 / R 6}(5), \\
v_{\mathrm{C}_{13 \mathrm{C} 15}}(5), v_{\mathrm{C} 10 \mathrm{C} 11}(5)\end{array}$ \\
\hline 77 & 1626.9 & 41.0 & 16.1 & $\begin{array}{l}v_{\mathrm{C} 14 \mathrm{C} 15}(14), v_{\mathrm{C}_{11} \mathrm{C} 12}(11), v_{\mathrm{O} 5 \mathrm{C} 9}(8),{ }^{3} \beta_{2 / R 6}(7), v_{\mathrm{C} 12 \mathrm{C} 13}(6), \rho_{\mathrm{N} 3 \mathrm{H} 23}(5), \\
v_{\mathrm{C} 6 \mathrm{C} 7}(5)\end{array}$ \\
\hline 78 & 1630.9 & 290.8 & 5.0 & $\begin{array}{l}v_{\mathrm{C} 6 \mathrm{C} 7}(16), v_{\mathrm{C} 8 \mathrm{C} 19}(6), v_{\mathrm{C} 14 \mathrm{C} 15}(6),{ }^{1} \beta_{3 / R 6}(6), v_{\mathrm{O} 4 \mathrm{C} 17}(6), \rho_{\mathrm{C} 7 \mathrm{H} 25}(5), \\
v_{\mathrm{C}_{12} \mathrm{C} 13}(5), v_{\mathrm{N} 3 \mathrm{C} 18}(5)\end{array}$ \\
\hline 79 & 1668.7 & 207.5 & 30.4 & $v_{\mathrm{O} 5 \mathrm{C} 9}(43),{ }^{2} \beta_{3 / R \sigma}(6),{ }^{2} \beta_{\text {trig/R6}}(6)$ \\
\hline 80 & 1720.8 & 180.4 & 30.9 & $v_{\mathrm{O} 4 \mathrm{C} 17}(71),{ }^{2} \beta_{\text {trig/R6}}(9)$ \\
\hline 81 & 2678.3 & 3.1 & 13.6 & $v_{\mathrm{S}_{1 \mathrm{H} 33}}(100)$ \\
\hline 82 & 3036.7 & 26.4 & 9.0 & $v_{\mathrm{C}_{2} \mathrm{H} 21}(77), v_{\mathrm{C}_{2} \mathrm{H} 22}(19)$ \\
\hline 83 & 3066.8 & 25.2 & 3.1 & $v_{\mathrm{C}_{20} \mathrm{H} 31}(49), v_{\mathrm{C} 20 \mathrm{H} 32}(48)$ \\
\hline 84 & 3081.5 & 7.2 & 4.9 & $v_{\mathrm{C}_{2} \mathrm{H} 22}(73), v_{\mathrm{C} 2 \mathrm{H} 21}(18), v_{\mathrm{C}_{20 \mathrm{H} 31}}(5)$ \\
\hline 85 & 3121.6 & 8.7 & 1.1 & $v_{\mathrm{C}_{2} 2 \mathrm{H} 32}(48), v_{\mathrm{C}_{20} \mathrm{H} 31}(44), v_{\mathrm{C}_{2} \mathrm{H} 22}(6)$ \\
\hline 86 & 3169.9 & 3.5 & 4.4 & $v_{\mathrm{C} 15 \mathrm{H} 29}(50), v_{\mathrm{C} 13 \mathrm{H} 27}(44)$ \\
\hline 87 & 3172.5 & 10.1 & 7.1 & $v_{\mathrm{C} 6 \mathrm{H} 24}(89), v_{\mathrm{C} 7 \mathrm{H} 25}(7)$ \\
\hline 88 & 3184.7 & 13.7 & 14.4 & $v_{\mathrm{C}_{13 \mathrm{H} 27}}(45), v_{\mathrm{C} 15 \mathrm{H} 29}(41), v_{\mathrm{C} 12 \mathrm{H} 26}(9)$ \\
\hline 89 & 3202.0 & 3.5 & 6.5 & $v_{\mathrm{C} 12 \mathrm{H} 26}(81), v_{\mathrm{C} 14 \mathrm{H} 28}(10), v_{\mathrm{C} 13 \mathrm{H} 27}(9)$ \\
\hline 90 & 3203.7 & 10.1 & 8.2 & $v_{\mathrm{C} 7 \mathrm{H} 25}(90), v_{\mathrm{C} 6 \mathrm{H} 24}(5)$ \\
\hline 91 & 3206.0 & 9.0 & 12.5 & $v_{\mathrm{C} 14 \mathrm{H} 28}(82), v_{\mathrm{C} 15 \mathrm{H} 29}(8), v_{\mathrm{C} 12 \mathrm{H} 26}(7)$ \\
\hline 92 & 3212.5 & 3.8 & 12.0 & $v_{\mathrm{C} 19 \mathrm{H} 30}(92)$ \\
\hline 93 & 3461.2 & 117.3 & 35.7 & $v_{\mathrm{N} 3 \mathrm{H} 23}(99)$ \\
\hline
\end{tabular}


Table S.5. Frequencies of normal vibrations, IR and Raman intensities and PEDs for ScTG conformer calculated at the B3LYP/6-311++G(d,p) level of theory.

\begin{tabular}{|c|c|c|c|c|}
\hline $\mathbf{N r}$ & $\begin{array}{l}\text { Freq. } \\
{\left[\mathrm{cm}^{-1}\right]}\end{array}$ & IR Int. & $\begin{array}{c}\text { Raman } \\
\text { Int. }\end{array}$ & PED $($ contributions $>5 \%)$ \\
\hline 1 & 22.8 & 0.5 & 7.3 & $\tau_{\mathrm{N} 3 \mathrm{C} 18}(40), \omega_{\mathrm{N} 3 \mathrm{H} 23}(31),{ }^{2} \tau_{3 / R 6}(9),{ }^{2} \tau_{2 / R 6}(6)$ \\
\hline 2 & 37.5 & 2.1 & 2.3 & ${ }^{2} \tau_{3 / R 6}(30),{ }^{1} \tau_{3 / R 6}(16), \tau_{\mathrm{C}_{2} \mathrm{~N} 3}(12), \tau_{\mathrm{C}_{2} \mathrm{C}_{20}}(9), \tau_{\mathrm{N} 3 \mathrm{C} 18}(9)$ \\
\hline 3 & 45.2 & 3.2 & 1.1 & ${ }^{2} \tau_{3 / R}(30), \tau_{\mathrm{C}_{2} \mathrm{C} 20}(18), \tau_{\mathrm{C}_{2} \mathrm{~N} 3}(13),{ }^{2} \tau_{2 / R 6}(11), \beta_{\mathrm{C}_{18 \mathrm{~N} 3 \mathrm{C} 2}}(6)$ \\
\hline 4 & 75.5 & 0.1 & 0.4 & ${ }^{2} \tau_{2 / R 6}(30), \omega_{\mathrm{N} 3 \mathrm{H} 23}(25),{ }^{1} \tau_{3 / R 6}(13)$ \\
\hline 5 & 103.0 & 0.8 & 0.4 & $\tau_{\mathrm{C} 2 \mathrm{~N} 3}(46), \tau_{\mathrm{C}_{2} \mathrm{C} 20}(25),{ }^{1} \tau_{2 / R 6}(7)$ \\
\hline 6 & 118.8 & 0.0 & 0.8 & ${ }^{2} \tau_{1 / R \sigma}(45),{ }^{3} \tau_{2 / R \sigma}(16),{ }^{2} \tau_{2 / R \sigma}(10),{ }^{3} \tau_{3 / R \sigma}(9)$ \\
\hline 7 & 160.9 & 1.7 & 0.3 & $\begin{array}{l}\rho_{\mathrm{C} 18 \mathrm{~N} 3}(21), \delta_{2 / \mathrm{C}_{20} \mathrm{H}_{31} \mathrm{H}_{32}}(12), v_{\mathrm{C} 9 \mathrm{C} 10}(6),{ }^{3} \beta_{2 / R 6}(5), v_{\mathrm{C}_{2} \mathrm{~N} 3}(5), \\
{ }^{1} \beta_{2 / \mathrm{R} 6}(5), \tau_{\mathrm{S}_{1} \mathrm{C} 20}(5)\end{array}$ \\
\hline 8 & 166.0 & 1.0 & 0.0 & ${ }^{4} b f l_{R R}(25),{ }^{2} b f l_{R R}(21), \tau_{N 3 C l 8}(10),{ }^{2} \tau_{3 / R 6}(7), \omega_{C 17 O 4}(6), \omega_{C 9 O 5}(6)$ \\
\hline 9 & 193.6 & 0.8 & 0.8 & ${ }^{1}{ }^{1} \tau_{2 / R 6}(27),{ }^{1} \tau_{3 / R 6}(24),{ }^{4} b f l_{R R}(10), \omega_{C l 9 H 30}(6),{ }^{2} \tau_{1 / R 6}(5),{ }^{2} \tau_{2 / R 6}(5)$ \\
\hline 10 & 220.1 & 9.0 & 0.3 & $\tau_{\mathrm{S} 1 \mathrm{C} 20_{2}}(75)$ \\
\hline 11 & 231.4 & 7.0 & 0.8 & $\delta_{2 / \mathrm{C} 20 \mathrm{H} 31 \mathrm{H} 32}(47), \tau_{\mathrm{S} 1 \mathrm{C} 20_{20}}(7), \delta_{2 / \mathrm{C} 2 \mathrm{H} 22 \mathrm{H} 21}(6)$ \\
\hline 12 & 239.1 & 2.0 & 0.8 & ${ }^{2} b f l_{R R}(27),{ }^{4} b f l_{R R}(11), \delta_{2 / C_{2} H_{22} H_{21}(}(9), \omega_{C 18 N 3}(7),{ }^{3} \tau_{3 / R 6}(6)$ \\
\hline 13 & 284.2 & 2.6 & 0.5 & $\rho_{\mathrm{C} 18 \mathrm{~N} 3}(18), \beta_{\mathrm{C}_{18 \mathrm{~N} 3 \mathrm{C} 2}}(11), \delta_{2 / \mathrm{C}_{20} \mathrm{H}_{31} \mathrm{H}_{32}}(10),{ }^{2} \beta_{2 / R 6}(9)$ \\
\hline 14 & 307.8 & 3.9 & 0.3 & $\begin{array}{l}\delta_{2 / \mathrm{C} 2 \mathrm{H} 22 \mathrm{H} 21}(16), \omega_{\mathrm{N}_{3} \mathrm{H}_{23}}(11), \beta_{\mathrm{C} 18 \mathrm{~N} 3 \mathrm{C} 2}(9), \delta_{2 / \mathrm{C}_{20} \mathrm{H}_{31} \mathrm{H}_{32}}(8),{ }^{2} b f l_{R R}(7), \\
{ }^{1} \tau_{3 / R 6}(6)\end{array}$ \\
\hline 15 & 345.1 & 1.5 & 0.1 & ${ }^{2} \beta_{3 / R 6}(26), \rho_{\mathrm{C} 17 \mathrm{O} 4}(20),{ }^{2} \beta_{2 / R 6}(14), \rho_{\mathrm{C} 18 \mathrm{~N} 3}(13)$ \\
\hline 16 & 366.1 & 7.2 & 0.8 & $\begin{array}{l}{ }^{2} \beta_{3 / R 6}(18),{ }^{2} \beta_{2 / R 6}(10), v_{\mathrm{C} 11 \mathrm{C} 17}(9),{ }^{1} \beta_{3 / R 6}(7), v_{\mathrm{C} 9 \mathrm{C} 16}(7), v_{\mathrm{C} 9 \mathrm{C} 10}(6), \\
\rho_{\mathrm{C} 17 \mathrm{O} 4}(6)\end{array}$ \\
\hline 17 & 412.7 & 0.7 & 0.3 & ${ }^{2} \beta_{2 / R 6}(22), \delta_{2 / \mathrm{C} 2 \mathrm{H} 22 \mathrm{H} 21}(8),{ }^{3} \beta_{2 / R 6}(8),{ }^{1} \beta_{2 / R 6}(7),{ }^{2} \beta_{3 / R 6}(6),{ }^{1} \beta_{3 / R 6}(5)$ \\
\hline 18 & 427.2 & 9.6 & 0.2 & ${ }^{3} \tau_{3 / R 6}(46),{ }^{4} b f l_{R R}(11), \rho_{C 9 O S}(9)$ \\
\hline 19 & 429.2 & 13.7 & 0.6 & ${ }^{3} \tau_{3 / R 6}(34), \rho_{\mathrm{C} 9 \mathrm{O} 5}(13),{ }^{4} b f l_{R R}(8), \rho_{\mathrm{Cl} 7 \mathrm{O} 4}(7)$ \\
\hline 20 & 432.9 & 0.3 & 0.0 & ${ }^{3} \tau_{2 / R 6}(33),{ }^{1} \tau_{2 / R 6}(20),{ }^{1} \tau_{3 / R 6}(16), \omega_{\mathrm{C} 9 \mathrm{O} 5}(6),{ }^{2} \tau_{1 / R 6}(6)$ \\
\hline 21 & 477.4 & 1.7 & 10.5 & ${ }^{2} \beta_{3 / R \sigma}(30),{ }^{2} \beta_{2 / R \sigma}(16),{ }^{1} \beta_{3 / R \sigma}(11),{ }^{3} \beta_{3 / R 6}(7), v_{\mathrm{C} 10 \mathrm{C} 11}(6)$ \\
\hline 22 & 490.5 & 1.8 & 0.0 & ${ }^{3} \tau_{2 / R 6}(23),{ }^{1} \tau_{3 / R 6}(22),{ }^{1} \tau_{2 / R 6}(11),{ }^{2} \tau_{2 / R 6}(9),{ }^{1} \tau_{1 / R 6}(6),{ }^{3} \tau_{3 / R 6}(5)$ \\
\hline 23 & 512.4 & 5.0 & 1.5 & $\begin{array}{l}{ }^{1} \beta_{2 / R \sigma}(16),{ }^{2} \beta_{2 / R \sigma}(10), v_{\mathrm{S}_{1} \mathrm{C} 20}(8),{ }^{1} \beta_{3 / R \sigma}(7), \rho_{\mathrm{C} 18 \mathrm{~N} 3}(6), \\
\delta_{2 / \mathrm{C} 2 \mathrm{H} 22 \mathrm{H} 21}(6),{ }^{3} \beta_{2 / R \sigma}(6)\end{array}$ \\
\hline 24 & 557.4 & 5.9 & 0.1 & $\omega_{\mathrm{C} 18 \mathrm{~N} 3}(30),{ }^{1} \tau_{3 / R 6}(13),{ }^{1} \tau_{2 / R \sigma}(13),{ }^{1} \tau_{1 / R 6}(10),{ }^{2} b f l_{R R}(6)$ \\
\hline 25 & 602.2 & 5.3 & 0.3 & $\begin{array}{l}{ }^{3} \beta_{2 / R 6}(14),{ }_{3 / R 6}(13), \beta_{\mathrm{C} 18 \mathrm{~N} 3 \mathrm{C} 2}(12),{ }^{1} \beta_{2 / R 6}(11), \rho_{\mathrm{C} 18 \mathrm{~N} 3}(8), \\
{ }^{2} \beta_{\text {trig/R6 }}(7),{ }^{3} \beta_{3 / R 6}(5)\end{array}$ \\
\hline 26 & 633.6 & 0.9 & 0.0 & ${ }^{2} \beta_{\text {trig/R6 }}(39),{ }_{2 / R 6}(16), \rho_{\mathrm{C} 18 \mathrm{~N} 3}(8), \beta_{\mathrm{C} 18 \mathrm{~N} 3 \mathrm{C} 2}(8)$ \\
\hline 27 & 670.1 & 0.3 & 0.1 & ${ }^{3} \tau_{1 / R 6}(39),{ }^{1} \tau_{1 / R 6}(14), \omega_{\mathrm{C} 9 \mathrm{O} 5}(13), \omega_{\mathrm{C} 18 \mathrm{~N} 3}(12)$ \\
\hline 28 & 673.2 & 13.7 & 1.7 & ${ }^{3} \beta_{3 / R \sigma}(42),{ }^{3} \beta_{2 / R \sigma}(11), v_{\mathrm{C} 10 \mathrm{C} 11}(8)$ \\
\hline 29 & 691.0 & 61.8 & 0.1 & $\tau_{\mathrm{N} 3 \mathrm{C} 18}(29), \omega_{\mathrm{N} 3 \mathrm{H} 23}(23), \tau_{\mathrm{C}_{2 \mathrm{~N} 3}}(13),{ }^{1} \tau_{1 / R 6}(11)$ \\
\hline 30 & 703.4 & 5.8 & 1.6 & $\begin{array}{l}\rho_{\mathrm{C} 9 \mathrm{O} 5}(17), \rho_{\mathrm{C} 17 \mathrm{O} 4}(15),{ }^{3} \beta_{2 / R 6}(14),{ }^{1} \beta_{2 / R 6}(9),{ }^{1} \beta_{3 / R 6}(8), v_{\mathrm{S} 1 \mathrm{C} 20}(6), \\
{ }^{3} \beta_{3 / R 6}(6), \rho_{\mathrm{C} 20 \mathrm{H} 31 \mathrm{H} 32}(5)\end{array}$ \\
\hline 31 & 722.5 & 1.2 & 1.1 & $\rho_{\mathrm{C} 20 \mathrm{H} 31 \mathrm{H} 32}(25), v_{\mathrm{S}_{1} \mathrm{C} 20}(21), \beta_{\mathrm{C}_{20} \mathrm{~S} 1 \mathrm{H} 33}(17), \delta_{2 / \mathrm{C}_{2} \mathrm{H} 22 \mathrm{H} 21}(6)$ \\
\hline 32 & 725.7 & 31.6 & 0.0 & ${ }^{3} \tau_{1 / R 6}(27),{ }^{1} \tau_{1 / R 6}(25), \omega_{\mathrm{C} 6 \mathrm{H} 24}(8), \omega_{\mathrm{C} 15 \mathrm{H} 29}(8), \omega_{\mathrm{C} 17 \mathrm{O} 4}(7)$ \\
\hline 33 & 747.9 & 66.3 & 0.0 & $\begin{array}{l}{ }_{\tau_{1 / R 6}}(15), \omega_{\mathrm{C} 18 \mathrm{~N} 3}(14), \omega_{\mathrm{C} 13 \mathrm{H} 27}(13), \omega_{\mathrm{C} 9 \mathrm{O} 5}(8),{ }^{3} \tau_{1 / R 6}(8), \\
\omega_{\mathrm{C} 17 \mathrm{O} 4}(7), \omega_{\mathrm{C} 14 \mathrm{H} 28}(7), \omega_{\mathrm{C} 15 \mathrm{H} 29}(6)\end{array}$ \\
\hline 34 & 754.8 & 3.3 & 1.4 & $v_{\mathrm{S}_{1} \mathrm{C}_{20}}(33), \rho_{\mathrm{C}_{20} \mathrm{H} 31 \mathrm{H} 32}(25), \rho_{\mathrm{C}_{2} \mathrm{H} 22 \mathrm{H} 21}(15), \delta_{2 / \mathrm{C} 2 \mathrm{H} 22 \mathrm{H} 21}(6)$ \\
\hline 35 & 787.1 & 5.5 & 0.0 & $\begin{array}{l}{ }^{3} \tau_{1 / R 6}(18), \omega_{\mathrm{C} 7 \mathrm{H} 25}(14),{ }^{2} \tau_{1 / R 6}(12), \omega_{\mathrm{C} 19 \mathrm{H} 30}(11), \omega_{\mathrm{C} 6 \mathrm{H} 24}(11), \\
\omega_{\mathrm{C} 17 \mathrm{O} 4}(9), \omega_{\mathrm{C} 955}(9)\end{array}$ \\
\hline 36 & 793.7 & 2.1 & 1.7 & $\begin{array}{l}{ }^{1} \beta_{\text {trig/R6 }}(18),{ }^{1} \beta_{3 / R \sigma}(15),{ }^{3} \beta_{2 / R 6}(13),{ }^{3} \beta_{\text {trig/R6 }}(9), v_{\mathrm{C} 9 \mathrm{C} 10}(7), \\
{ }^{2} \beta_{\text {trig/R6 }}(5)\end{array}$ \\
\hline
\end{tabular}




\begin{tabular}{|c|c|c|c|c|}
\hline 37 & 810.0 & 0.8 & 0.1 & $\begin{array}{l}\omega_{\mathrm{C} 9 \mathrm{O} 5}(27), \omega_{\mathrm{C} 13 \mathrm{H} 27}(14),{ }^{1} \tau_{1 / R 6}(11), \omega_{\mathrm{C} 18 \mathrm{~N} 3}(9),{ }^{2} \tau_{1 / R 6}(8), \\
\omega_{\mathrm{C} 15 \mathrm{H} 29}(7)\end{array}$ \\
\hline 38 & 826.0 & 25.8 & 0.0 & $\begin{array}{l}\omega_{\mathrm{C} 7 \mathrm{H} 25}(23), \omega_{\mathrm{C} 17 \mathrm{O} 4}(18),{ }^{1} \tau_{1 / R 6}(16), \omega_{\mathrm{C} 18 \mathrm{~N} 3}(9),{ }^{2} \tau_{1 / R 6}(7), \\
\omega_{\mathrm{C} 15 \mathrm{H} 29}(6), \omega_{\mathrm{C} 6 \mathrm{H} 24}(5)\end{array}$ \\
\hline 39 & 865.5 & 11.2 & 2.8 & $\beta_{\mathrm{C}_{20 \mathrm{~S} 1 \mathrm{H} 33}}(21), \rho_{\mathrm{C} 2 \mathrm{H} 22 \mathrm{H} 21}(12), \tau_{\mathrm{C} 20 \mathrm{H} 31 \mathrm{H} 32}(8), v_{\mathrm{C} 16 \mathrm{C} 18}(6),{ }^{1} \beta_{2 / R 6}(5)$ \\
\hline 40 & 880.6 & 13.3 & 0.6 & $\beta_{\mathrm{C}_{20 \mathrm{~S} 1 \mathrm{H} 33}}(28), \tau_{\mathrm{C} 20 \mathrm{H} 31 \mathrm{H} 32}(11), \rho_{\mathrm{C} 2 \mathrm{H} 22 \mathrm{H} 21}(10), v_{\mathrm{C} 7 \mathrm{C} 18}(6)$ \\
\hline 41 & 910.7 & 0.8 & 0.0 & $\omega_{\mathrm{C} 19 \mathrm{H} 30}(33), \omega_{\mathrm{C} 7 \mathrm{H} 25}(22), \omega_{\mathrm{C} 12 \mathrm{H} 26}(11), \omega_{\mathrm{C} 14 \mathrm{H} 28}(11), \omega_{\mathrm{C} 15 \mathrm{H} 29}(6)$ \\
\hline 42 & 917.8 & 0.6 & 0.0 & $\begin{array}{l}\omega_{\mathrm{C} 14 \mathrm{H} 28}(20), \omega_{\mathrm{C} 12 \mathrm{H} 26}(18), \omega_{\mathrm{C} 19 \mathrm{H} 30}(17), \omega_{\mathrm{C} 7 \mathrm{H} 25}(11), \omega_{\mathrm{C} 13 \mathrm{H} 27}(10), \\
\omega_{\mathrm{C} 15 \mathrm{H} 29}(7)\end{array}$ \\
\hline 43 & 928.9 & 2.5 & 1.2 & ${ }^{1} \beta_{\text {trig/R6 } 6}(25),{ }^{3} \beta_{\text {trig/R6 }}(24), \rho_{\mathrm{C} 9 \mathrm{O} 5}(14), v_{\mathrm{C} 16 \mathrm{C} 18}(7), \rho_{\mathrm{C} 17 \mathrm{O} 4}(5)$ \\
\hline 44 & 994.3 & 83.1 & 2.0 & $\begin{array}{l}v_{\mathrm{C} 2 \mathrm{~N} 3}(16), v_{\mathrm{C} 8 \mathrm{C} 17}(10), v_{\mathrm{C}_{2} \mathrm{C} 20}(9), \rho_{\mathrm{C} 17 \mathrm{O} 4}(7), v_{\mathrm{C}_{11 C} 12}(7), v_{\mathrm{C} 8 \mathrm{C} 19}(6), \\
\rho_{\mathrm{C} 9 \mathrm{O} 5}(5)\end{array}$ \\
\hline 45 & 999.6 & 0.1 & 0.0 & $\omega_{\mathrm{C} 6 \mathrm{H} 24}(50), \omega_{\mathrm{C} 19 \mathrm{H} 30}(22), \omega_{\mathrm{C} 7 \mathrm{H} 25}(10),{ }^{1} \tau_{1 / R 6}(7)$ \\
\hline 46 & 1002.7 & 2.2 & 0.0 & $\omega_{\mathrm{C} 12 \mathrm{H} 26}(32), \omega_{\mathrm{C} 14 \mathrm{H} 28}(29), \omega_{\mathrm{C} 13 \mathrm{H} 27}(11), \omega_{\mathrm{C} 15 \mathrm{H} 29}(10)$ \\
\hline 47 & 1016.6 & 0.0 & 0.0 & $\omega_{\mathrm{C} 15 \mathrm{H} 29}(30), \omega_{\mathrm{C} 13 \mathrm{H} 27}(28), \omega_{\mathrm{C} 14 \mathrm{H} 28}(15), \omega_{\mathrm{C} 12 \mathrm{H} 26}(13),{ }^{3} \tau_{1 / R 6}(12)$ \\
\hline 48 & 1020.7 & 20.1 & 0.6 & $v_{\mathrm{C} 2 \mathrm{C} 20}(66)$ \\
\hline 49 & 1056.5 & 1.1 & 6.4 & $v_{\mathrm{C}_{13} \mathrm{C} 15}(34), v_{\mathrm{C} 14 \mathrm{C} 15}(13), v_{\mathrm{C}_{12} \mathrm{C} 13}(11), \rho_{\mathrm{C} 12 \mathrm{H} 26}(11), \rho_{\mathrm{C} 14 \mathrm{H} 28}(11)$ \\
\hline 50 & 1082.2 & 7.4 & 0.7 & $\rho_{\mathrm{C}_{2} \mathrm{H} 22 \mathrm{H} 21}(23), \rho_{\mathrm{C} 20 \mathrm{H} 31 \mathrm{H} 32}(18), \beta_{\mathrm{C}_{20 \mathrm{~S} 1 \mathrm{H} 33}}(17), v_{\mathrm{C} 7 \mathrm{C} 18}(6)$ \\
\hline 51 & 1097.9 & 31.8 & 0.7 & $v_{\mathrm{C} 6 \mathrm{C} 19}(25),{ }^{\mathrm{I}} \beta_{\text {trig/R6}}(16), \rho_{\mathrm{C} 19 \mathrm{H} 30}(14), v_{\mathrm{C} 2 \mathrm{~N} 3}(11)$ \\
\hline 52 & 1113.1 & 1.6 & 0.3 & $\begin{array}{l}{ }^{3} \beta_{\text {trig/R6 }}(38), v_{\mathrm{C} 12 \mathrm{C} 13}(8), v_{\mathrm{C} 14 \mathrm{C} 15}(8), \rho_{\mathrm{C} 13 \mathrm{H} 27}(8), \rho_{\mathrm{C} 15 \mathrm{H} 29}(7), \\
v_{\mathrm{C} 9 \mathrm{C} 10}(6), v_{\mathrm{C}_{11} \mathrm{C}_{17}}(5), \rho_{\mathrm{C} 14 \mathrm{H} 28}(5)\end{array}$ \\
\hline 53 & 1133.8 & 1.1 & 3.2 & $v_{\mathrm{C} 2 \mathrm{~N} 3}(38), \rho_{\mathrm{C} 18 \mathrm{~N} 3}(7), \tau_{\mathrm{C} 20 \mathrm{H} 31 \mathrm{H} 32}(6), v_{\mathrm{C} 8 \mathrm{C} 17}(6)$ \\
\hline 54 & 1175.3 & 11.3 & 4.5 & $\begin{array}{l}\rho_{\mathrm{C} 13 \mathrm{H} 27}(15), \rho_{\mathrm{C} 12 \mathrm{H} 26}(12), v_{\mathrm{C} 9 \mathrm{C} 16}(10),{ }^{1} \beta_{\text {trig/R6 }}(7), v_{\mathrm{C} 9 \mathrm{C} 10}(7), \\
{ }^{3} \beta_{\text {trig/R6}}(6), v_{\mathrm{C} 7 \mathrm{C} 18}(5)\end{array}$ \\
\hline 55 & 1183.0 & 22.5 & 0.2 & $\rho_{\mathrm{C} 15 \mathrm{H} 29}(31), \rho_{\mathrm{C} 14 \mathrm{H} 28}(11), \rho_{\mathrm{C} 13 \mathrm{H} 27}(10), v_{\mathrm{C} 14 \mathrm{C} 15}(8), v_{\mathrm{C} 13 \mathrm{C} 15}(6)$ \\
\hline 56 & 1197.4 & 22.8 & 9.2 & $\rho_{\mathrm{C} 6 \mathrm{H} 24}(29), \rho_{\mathrm{C} 7 \mathrm{H} 25}(13), v_{\mathrm{C} 6 \mathrm{C} 7}(7), \rho_{\mathrm{C} 13 \mathrm{H} 27}(6)$ \\
\hline 57 & 1213.3 & 12.6 & 5.2 & $\rho_{\mathrm{C} 19 \mathrm{H} 30}(21), \rho_{\mathrm{C} 7 \mathrm{H} 25}(15), v_{\mathrm{C} 8 \mathrm{C} 19}(9), \tau_{\mathrm{C} 20 \mathrm{H} 31 \mathrm{H} 32}(7), \rho_{\mathrm{C} 6 \mathrm{H} 24}(7)$ \\
\hline 58 & 1222.2 & 75.1 & 0.3 & $\tau_{\mathrm{C} 20 \mathrm{H} 31 \mathrm{H} 32}(27), \tau_{\mathrm{C} 2 \mathrm{H} 22 \mathrm{H} 21}(12), \rho_{\mathrm{C} 2 \mathrm{H} 22 \mathrm{H} 21}(6), \rho_{\mathrm{C} 19 \mathrm{H} 30}(5)$ \\
\hline 59 & 1265.1 & 23.1 & 2.2 & $\rho_{\mathrm{C} 12 \mathrm{H} 26}(16), \rho_{\mathrm{C} 14 \mathrm{H} 28}(10),{ }^{2} \beta_{\text {trig/R6 }}(10), v_{\mathrm{C} 11 \mathrm{C} 12}(7), \tau_{\mathrm{C} 20 \mathrm{H} 31 \mathrm{H} 32}(7)$ \\
\hline 60 & 1280.5 & 68.5 & 0.2 & $\omega_{\mathrm{C} 20 \mathrm{H} 31 \mathrm{H} 32}(77), \omega_{\mathrm{C} 2 \mathrm{H} 22 \mathrm{H} 21}(12)$ \\
\hline 61 & 1289.8 & 574.0 & 8.6 & $\begin{array}{l}v_{\mathrm{C} 9 \mathrm{C} 10}(13), v_{\mathrm{C} 8 \mathrm{C} 16}(8), v_{\mathrm{C} 9 \mathrm{C} 16}(8), \rho_{\mathrm{C} 14 \mathrm{H} 28}(7), v_{\mathrm{C} 7 \mathrm{C} 18}(6), \rho_{\mathrm{C} 19 \mathrm{H} 30}(6), \\
v_{\mathrm{C} 8 \mathrm{C} 17}(6), v_{\mathrm{C} 6 \mathrm{C} 19}(5), v_{\mathrm{C} 16 \mathrm{C} 18}(5)\end{array}$ \\
\hline 62 & 1316.1 & 136.4 & 1.5 & $v_{\mathrm{C} 11 \mathrm{C} 17}(19), v_{\mathrm{C} 8 \mathrm{C} 17}(13), \rho_{\mathrm{C} 12 \mathrm{H} 26}(9), \rho_{\mathrm{C} 17 \mathrm{O} 4}(7),{ }^{2} \beta_{2 / R 6}(6)$ \\
\hline 63 & 1327.8 & 66.6 & 6.5 & $\begin{array}{l}\tau_{\mathrm{C} 2 \mathrm{H} 22 \mathrm{H} 21}(28), v_{\mathrm{N} 3 \mathrm{C} 18}(16),{ }^{1} \beta_{\text {trig/R6 }}(8), v_{\mathrm{C} 16 \mathrm{C} 18}(7), \tau_{\mathrm{C} 20 \mathrm{H} 31 \mathrm{H} 32}(6), \\
\rho_{\mathrm{N} 3 \mathrm{H} 23}(6)\end{array}$ \\
\hline 64 & 1338.2 & 136.2 & 2.2 & $\begin{array}{l}\tau_{\mathrm{C} 2 \mathrm{H} 22 \mathrm{H} 21}(22), v_{\mathrm{N} 3 \mathrm{C} 18}(11), \rho_{\mathrm{N} 3 \mathrm{H} 23}(10), v_{\mathrm{C} 16 \mathrm{C} 18}(8), v_{\mathrm{C} 9 \mathrm{C} 16}(8), \\
\rho_{\mathrm{C} 6 \mathrm{H} 24}(6), \tau_{\mathrm{C}_{20} \mathrm{H} 31 \mathrm{H} 32}(5)\end{array}$ \\
\hline 65 & 1358.6 & 16.2 & 1.0 & $\begin{array}{l}v_{\mathrm{C} 10 \mathrm{C} 14}(15), v_{\mathrm{C} 10 \mathrm{C} 11}(15), v_{\mathrm{C} 11 \mathrm{C} 12}(14), v_{\mathrm{C} 13 \mathrm{C} 15}(14), v_{\mathrm{C} 12 \mathrm{C} 13}(13), \\
v_{\mathrm{C} 14 \mathrm{C} 15}(13)\end{array}$ \\
\hline 66 & 1379.1 & 16.3 & 1.6 & $\begin{array}{l}v_{\mathrm{C} 16 \mathrm{C} 18}(17), v_{\mathrm{C} 8 \mathrm{C} 19}(11), v_{\mathrm{C} 8 \mathrm{C} 16}(10), v_{\mathrm{C} 9 \mathrm{C} 16}(9), v_{\mathrm{C} 6 \mathrm{C} 19}(7), v_{\mathrm{C} 6 \mathrm{C} 7}(6), \\
v_{\mathrm{C} 7 \mathrm{C} 18}(6), \rho_{\mathrm{C} 9 \mathrm{O} 5}(6), v_{\mathrm{C} 9 \mathrm{C} 10}(5)\end{array}$ \\
\hline 67 & 1389.8 & 16.6 & 2.0 & $\omega_{\mathrm{C} 2 \mathrm{H} 22 \mathrm{H} 21}(70), \omega_{\mathrm{C} 20 \mathrm{H} 31 \mathrm{H} 32}(7)$ \\
\hline 68 & 1433.8 & 31.7 & 0.4 & $\rho_{\mathrm{C} 7 \mathrm{H} 25}(25), v_{\mathrm{C} 6 \mathrm{C} 7}(11), v_{\mathrm{C} 8 \mathrm{C} 19}(11), v_{\mathrm{C} 8 \mathrm{C} 16}(6), v_{\mathrm{C} 9 \mathrm{C} 16}(6)$ \\
\hline 69 & 1476.1 & 3.0 & 5.1 & $\delta_{1 / \mathrm{C}_{20} \mathrm{H} 31 \mathrm{H} 32}(62), \delta_{1 / \mathrm{C}_{2} \mathrm{H} 22 \mathrm{H} 21}(8)$ \\
\hline 70 & 1479.3 & 1.9 & 1.6 & $\delta_{1 / \mathrm{C} 20 \mathrm{H} 31 \mathrm{H} 32}(22), \rho_{\mathrm{C}_{15} \mathrm{H} 29}(13), \rho_{\mathrm{C} 13 \mathrm{H} 27}(12), v_{\mathrm{C} 10 \mathrm{C} 14}(6), v_{\mathrm{C}_{11} \mathrm{C} 12}(6)$ \\
\hline 71 & 1487.9 & 11.1 & 0.8 & $\begin{array}{l}\rho_{\mathrm{C} 19 \mathrm{H} 30}(11), v_{\mathrm{C} 8 \mathrm{C} 16}(9), \rho_{\mathrm{C} 6 \mathrm{H} 24}(7), \rho_{\mathrm{C} 13 \mathrm{H} 27}(7), \rho_{\mathrm{C} 15 \mathrm{H} 29}(5), \\
\delta_{1 / \mathrm{C}_{2} \mathrm{H} 22 \mathrm{H} 21}(5), v_{\mathrm{C} 7 \mathrm{C} 18}(5)\end{array}$ \\
\hline 72 & 1505.2 & 3.5 & 1.1 & $\delta_{1 / \mathrm{C}_{2} \mathrm{H}_{22} \mathrm{H}_{21}(65)}$ \\
\hline 73 & 1508.2 & 5.0 & 1.8 & $\rho_{\mathrm{C} 14 \mathrm{H} 28}(16), \rho_{\mathrm{C} 12 \mathrm{H} 26}(15), \delta_{1 / \mathrm{C} 2 \mathrm{H} 22 \mathrm{H} 21}(14), v_{\mathrm{C} 10 \mathrm{C} 11}(13), v_{\mathrm{C}_{13} \mathrm{C} 15}(7)$ \\
\hline
\end{tabular}




\begin{tabular}{|l|l|l|l|l|}
\hline 74 & 1552.9 & 201.5 & 2.1 & $v_{\mathrm{C} 6 \mathrm{C} 19}(19), \rho_{\mathrm{N} 3 \mathrm{H} 23}(18), v_{\mathrm{N}_{3} \mathrm{C} 18}(16), \rho_{\mathrm{C} 6 \mathrm{H} 24}(12), v_{\mathrm{C} 6 \mathrm{C} 7}(7), v_{\mathrm{C} 8 \mathrm{C} 16}(6)$ \\
\hline 75 & 1611.0 & 34.0 & 11.0 & $\begin{array}{l}v_{\mathrm{C} 13 \mathrm{C} 15}(14), v_{\mathrm{C} 10 \mathrm{C} 11}(14), v_{\mathrm{O} 5 \mathrm{C} 9}(10), v_{\mathrm{C} 12 \mathrm{C} 13}(7), \beta_{3 / R 6}(6), \\
v_{\mathrm{C} 10 \mathrm{C} 14}(6), \rho_{\mathrm{C} 13 \mathrm{H} 27}(5), v_{\mathrm{C} 8 \mathrm{C} 19}(5)\end{array}$ \\
\hline 76 & 1614.3 & 92.7 & 7.9 & $\begin{array}{l}\rho_{\mathrm{N} 3 \mathrm{H} 23}(21), v_{\mathrm{C} 6 \mathrm{C} 19}(9), v_{\mathrm{C} 8 \mathrm{C} 19}(8), v_{\mathrm{C} 16 \mathrm{C} 18}(8), v_{\mathrm{C} 7 \mathrm{C} 18}(7),{ }^{1} \beta_{2 / R 6}(6), \\
v_{\mathrm{C} 13 \mathrm{C} 15}(5), v_{\mathrm{C} 10 \mathrm{C} 11}(5)\end{array}$ \\
\hline 77 & 1626.8 & 43.7 & 16.5 & $\begin{array}{l}v_{\mathrm{C} 14 \mathrm{C} 15}(14), v_{\mathrm{C} 11 \mathrm{C} 12}(11), v_{\mathrm{O} 5 \mathrm{C} 9}(8),{ }^{3} \beta_{2 / R 6}(7), v_{\mathrm{C} 12 \mathrm{C} 13}(6), v_{\mathrm{C} 6 \mathrm{C} 7}(6), \\
\rho_{\mathrm{N} 3 \mathrm{H} 23}(5)\end{array}$ \\
\hline 78 & 1630.8 & 288.4 & 5.4 & $\begin{array}{l}v_{\mathrm{C} 6 \mathrm{C} 7}(15), v_{\mathrm{C} 14 \mathrm{C} 15}(7), v_{\mathrm{C} 8 \mathrm{C} 19}(6), v_{\mathrm{O} 4 \mathrm{C} 17}(6),{ }^{1} \beta_{3 / R 6}(6), v_{\mathrm{C} 12 \mathrm{C} 13}(6), \\
\rho_{\mathrm{C} 7 \mathrm{H} 25}(5)\end{array}$ \\
\hline 79 & 1668.7 & 206.6 & 32.8 & $v_{\mathrm{O} 5 \mathrm{C} 9}(43),{ }^{2} \beta_{3 / R 6}(6),{ }^{2} \beta_{t r i g / R 6}(6)$ \\
\hline 80 & 1720.9 & 180.5 & 32.9 & $v_{\mathrm{O} 4 \mathrm{C} 17}(71),{ }^{2} \beta_{t r i g / R 6}(9)$ \\
\hline 81 & 2663.4 & 6.4 & 7.7 & $v_{\mathrm{S} 1 \mathrm{H} 33}(100)$ \\
\hline 83 & 3036.7 & 32.5 & 7.1 & $v_{\mathrm{C} 2 \mathrm{H} 21}(83), v_{\mathrm{C} 2 \mathrm{H} 22}(14)$ \\
\hline 84 & 3069.4 & 17.6 & 3.0 & $v_{\mathrm{C} 20 \mathrm{H} 32}(49), v_{\mathrm{C} 20 \mathrm{H} 31}(49)$ \\
\hline 85 & 3120.8 & 5.6 & 5.2 & $v_{\mathrm{C} 2 \mathrm{H} 22}(75), v_{\mathrm{C} 2 \mathrm{H} 21}(11), v_{\mathrm{C} 20 \mathrm{H} 31}(8), v_{\mathrm{C} 20 \mathrm{H} 32}(5)$ \\
\hline 86 & 3170.0 & 3.4 & 4.3 & $v_{\mathrm{C} 15 \mathrm{H} 29}(49), v_{\mathrm{C} 13 \mathrm{H} 27}(45), v_{\mathrm{C} 2 \mathrm{H} 22}(10)$ \\
\hline 87 & 3172.3 & 10.2 & 7.1 & $v_{\mathrm{C} 6 \mathrm{H} 24}(89), v_{\mathrm{C} 7 \mathrm{H} 25}(7)$ \\
\hline 88 & 3184.8 & 13.7 & 14.4 & $v_{\mathrm{C} 13 \mathrm{H} 27}(43), v_{\mathrm{C} 15 \mathrm{H} 29}(42), v_{\mathrm{C} 12 \mathrm{H} 26}(8), v_{\mathrm{C} 14 \mathrm{H} 28}(5)$ \\
\hline 89 & 3202.0 & 3.6 & 6.5 & $v_{\mathrm{C} 12 \mathrm{H} 26}(82), v_{\mathrm{C} 14 \mathrm{H} 28}(9), v_{\mathrm{C} 13 \mathrm{H} 27}(8)$ \\
\hline 90 & 3204.0 & 14.2 & 8.1 & $v_{\mathrm{C} 7 \mathrm{H} 25}(90), v_{\mathrm{C} 6 \mathrm{H} 24}(5)$ \\
\hline 91 & 3206.1 & 8.8 & 12.4 & $v_{\mathrm{C} 14 \mathrm{H} 28}(83), v_{\mathrm{C} 15 \mathrm{H} 29}(8), v_{\mathrm{C} 12 \mathrm{H} 26}(7)$ \\
\hline 92 & 3212.7 & 4.2 & 11.9 & $v_{\mathrm{C} 19 \mathrm{H} 30}(92)$ \\
\hline 93 & 3458.7 & 117.4 & 37.4 & $v_{\mathrm{N} 3 \mathrm{H} 23}(99)$ \\
\hline
\end{tabular}


Table S.6. Frequencies of normal vibrations, IR and Raman intensities and PEDs for ScT-G conformer calculated at the B3LYP/6-311++G(d,p) level of theory.

\begin{tabular}{|c|c|c|c|c|}
\hline $\mathbf{N r}$ & $\begin{array}{l}\text { Freq. } \\
{\left[\mathrm{cm}^{-1}\right]}\end{array}$ & IR Int. & $\begin{array}{c}\text { Raman } \\
\text { Int. }\end{array}$ & PED $($ contributions $>5 \%)$ \\
\hline 1 & 24.6 & 0.3 & 7.5 & $\tau_{\mathrm{N} 3 \mathrm{C} 18}(40), \omega_{\mathrm{N} 3 \mathrm{H} 23}(29),{ }^{2} \tau_{3 / R 6}(11),{ }^{2} \tau_{2 / R 6}(8)$ \\
\hline 2 & 35.2 & 0.8 & 3.1 & $\tau_{\mathrm{C}_{2} \mathrm{C} 20}(25), \tau_{\mathrm{C} 2 \mathrm{~N} 3}(16),{ }^{2} \tau_{3 / R 6}(16),{ }^{1} \tau_{3 / R 6}(10), \beta_{\mathrm{C}_{18 \mathrm{~N} 3 \mathrm{C} 2}}(9), \tau_{\mathrm{N} 3 \mathrm{C} 18}(7)$ \\
\hline 3 & 44.3 & 3.0 & 0.8 & ${ }^{2} \tau_{3 / R 6}(40), \tau_{\mathrm{C}_{2} \mathrm{C} 20}(11),{ }^{2} \tau_{2 / R 6}(10),{ }^{1} \tau_{3 / R 6}(8), \omega_{\mathrm{N} 3 \mathrm{H} 23}(6)$ \\
\hline 4 & 75.0 & 1.0 & 0.3 & ${ }^{2} \tau_{2 / R 6}(30), \omega_{\mathrm{N} 3 \mathrm{H} 23}(25),{ }^{1} \tau_{3 / R 6}(13), \tau_{\mathrm{C} 2 \mathrm{~N} 3}(6)$ \\
\hline 5 & 103.6 & 2.7 & 0.4 & $\tau_{\mathrm{C}_{2} \mathrm{~N} 3}(47), \tau_{\mathrm{C} 2 \mathrm{C} 20}(20),{ }^{1} \tau_{2 / R 6}(8),{ }^{2} \tau_{2 / R \sigma}(5)$ \\
\hline 6 & 119.0 & 0.2 & 0.8 & ${ }^{2} \tau_{1 / R \sigma}(45),{ }^{3} \tau_{2 / R \sigma}(17),{ }^{2} \tau_{2 / R \sigma}(10),{ }^{3} \tau_{3 / R \sigma}(8)$ \\
\hline 7 & 163.9 & 0.8 & 0.4 & $\begin{array}{l}\rho_{\mathrm{C} 18 \mathrm{~N} 3}(19), \delta_{2 / \mathrm{C} 20 \mathrm{H} 31 \mathrm{H} 32}(12), v_{\mathrm{C} 9 \mathrm{C} 10}(6), \tau_{\mathrm{C} 2 \mathrm{~N} 3}(6),{ }^{3} \beta_{2 / R 6}(6), \\
{ }^{1} \beta_{2 / R 6}(5)\end{array}$ \\
\hline 8 & 166.7 & 0.3 & 0.1 & ${ }^{4} b f l_{R R}(26),{ }^{2} b f l_{R R}(20), \tau_{N 3 C l 8}(10),{ }^{2} \tau_{3 / R 6}(7), \omega_{C l 7 O 4}(6), \omega_{C 9 O 5}(6)$ \\
\hline 9 & 194.6 & 0.5 & 0.9 & ${ }^{1}{ }^{1} \tau_{2 / R 6}(27),{ }^{1} \tau_{3 / R \sigma}(24),{ }^{4} b f l_{R R}(10), \omega_{C 19 H 30}(6),{ }^{2} \tau_{1 / R 6}(5),{ }^{2} \tau_{2 / R 6}(5)$ \\
\hline 10 & 209.3 & 9.9 & 0.7 & $\tau_{\mathrm{S} 1 \mathrm{C} 20}(59), \delta_{2 / \mathrm{C}_{20} \mathrm{H} 31 \mathrm{H} 32}(18)$ \\
\hline 11 & 238.1 & 4.1 & 0.7 & $\begin{array}{l}{ }^{2} b f l_{R R}(27), \delta_{2 / C_{2} H_{22} H_{21}}(14),{ }^{4} b f l_{R R}(12), \delta_{2 / C 2 O H 31 H 32}(8), \omega_{C 18 N 3}(7), \\
{ }^{3} \tau_{3 / R 6}(6)\end{array}$ \\
\hline 12 & 246.9 & 6.7 & 0.3 & $\tau_{\mathrm{S} 1 \mathrm{C} 20}(29), \delta_{2 / \mathrm{C} 20 \mathrm{H} 31 \mathrm{H} 32}(20),{ }^{2} \beta_{2 / R 6}(7), v_{\mathrm{C} 8 \mathrm{C} 17}(5)$ \\
\hline 13 & 289.4 & 5.8 & 0.5 & $\begin{array}{l}\delta_{2 / \mathrm{C} 20 \mathrm{H} 31 \mathrm{H} 32}(16), \rho_{\mathrm{C} 18 \mathrm{~N} 3_{3}}(15), \beta_{\mathrm{C} 18 \mathrm{~N} 3 \mathrm{C} 2_{2}}(9),{ }^{2} \beta_{2 / R 6}(8), \delta_{2 / \mathrm{C}_{2} \mathrm{H}_{22} \mathrm{H}_{21}}(5), \\
\tau_{\mathrm{S}_{1} \mathrm{C} 20}(5)\end{array}$ \\
\hline 14 & 309.3 & 1.7 & 0.3 & $\begin{array}{l}\delta_{2 / \mathrm{C}_{2} \mathrm{H} 22 \mathrm{H} 21}(16), \omega_{\mathrm{N}_{3} \mathrm{H} 23}(11), \beta_{\mathrm{C} 18 \mathrm{~N} 3 \mathrm{C} 2_{2}}(10),{ }^{2} b f l_{R R}(6),{ }^{I} \tau_{3 / R 6}(6), \\
\delta_{2 / \mathrm{C} 2 \mathrm{OH} 31 \mathrm{H} 32}(5), \tau_{\mathrm{C}_{2 N} 3}(5)\end{array}$ \\
\hline 15 & 345.5 & 1.6 & 0.1 & ${ }^{2} \beta_{3 / R 6}(26), \rho_{\mathrm{C} 17 \mathrm{O} 4}(19), \rho_{\mathrm{C} 18 \mathrm{~N} 3}(14),{ }^{2} \beta_{2 / R 6}(14)$ \\
\hline 16 & 366.6 & 6.8 & 0.8 & $\begin{array}{l}{ }^{2} \beta_{3 / R 6}(18),{ }^{2} \beta_{2 / R 6}(10), v_{\mathrm{C} 11 \mathrm{C} 17}(9),{ }^{1} \beta_{3 / R 6}(8), v_{\mathrm{C} 9 \mathrm{C} 16}(7), \rho_{\mathrm{C} 17 \mathrm{O} 4}(7), \\
v_{\mathrm{C} 9 \mathrm{C} 10}(6)\end{array}$ \\
\hline 17 & 412.0 & 0.8 & 0.4 & $\begin{array}{l}{ }^{2} \beta_{2 / R \sigma}(22), \delta_{2 / \mathrm{C}_{2} \mathrm{H} 22 \mathrm{H} 21}(8),{ }^{3} \beta_{2 / R \sigma}(8),{ }^{1} \beta_{2 / R \sigma}(6),{ }^{2} \beta_{3 / R \sigma}(6),{ }^{1} \beta_{3 / R \sigma}(6), \\
v_{\mathrm{S}_{1} \mathrm{C} 20}(5)\end{array}$ \\
\hline 18 & 427.3 & 11.0 & 0.3 & ${ }^{3} \tau_{3 / R 6}(44), \rho_{\mathrm{C} 9 \mathrm{O} 5}(11),{ }^{4} b f l_{R R}(11)$ \\
\hline 19 & 429.5 & 12.5 & 0.5 & ${ }^{3} \tau_{3 / R 6}(38), \rho_{\mathrm{C} 9 \mathrm{O} 5}(11),{ }^{4} b f l_{R R}(8), \rho_{\mathrm{Cl}_{7104}}(6)$ \\
\hline 20 & 432.8 & 0.6 & 0.1 & ${ }^{3} \tau_{2 / R 6}(33),{ }^{1} \tau_{2 / R 6}(20),{ }^{1} \tau_{3 / R 6}(15), \omega_{\mathrm{C} 9 \mathrm{O} 5}(6),{ }^{2} \tau_{1 / R 6}(6)$ \\
\hline 21 & 477.3 & 1.9 & 10.5 & ${ }^{2} \beta_{3 / R 6}(31),{ }^{2} \beta_{2 / R 6}(16),{ }^{1} \beta_{3 / R 6}(11),{ }^{3} \beta_{3 / R 6}(7), v_{\mathrm{C} 10 \mathrm{C} 11}(6)$ \\
\hline 22 & 489.8 & 1.8 & 0.0 & ${ }^{3} \tau_{2 / R \sigma}(23),{ }^{1} \tau_{3 / R \sigma}(22),{ }^{1} \tau_{2 / R \sigma}(11),{ }^{2} \tau_{2 / R 6}(9),{ }^{1} \tau_{1 / R \sigma}(6),{ }^{3} \tau_{3 / R \sigma}(6)$ \\
\hline 23 & 510.8 & 4.9 & 1.4 & $\begin{array}{l}{ }^{1} \beta_{2 / R 6}(16),{ }^{2} \beta_{2 / R 6}(10), v_{\mathrm{S} 1 \mathrm{C} 20}(8), \rho_{\mathrm{C} 18 \mathrm{~N} 3}(6),{ }^{1} \beta_{3 / R 6}(6),{ }^{3} \beta_{2 / R 6}(6), \\
\delta_{2 / \mathrm{C} 2 \mathrm{H} 22 \mathrm{H} 21}(5)\end{array}$ \\
\hline 24 & 557.8 & 5.8 & 0.1 & $\omega_{\mathrm{C} 18 \mathrm{~N} 3}(29),{ }^{1} \tau_{3 / R \sigma}(13),{ }^{\mathrm{I}}{ }{ }_{2 / R \sigma}(13),{ }^{1}{ }_{1 / R \sigma}(10),{ }^{2} b f l_{R R}(6)$ \\
\hline 25 & 602.2 & 6.2 & 0.3 & 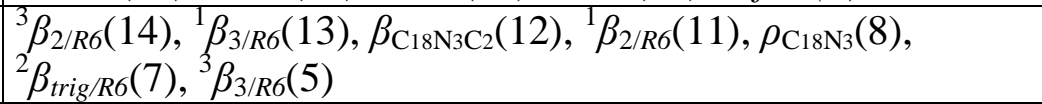 \\
\hline 26 & 633.2 & 1.3 & 0.0 & ${ }^{2} \beta_{\text {trig/R6 }}(39),{ }_{2 / R 6}^{1}(16), \rho_{\mathrm{C} 18 \mathrm{~N} 3}(8), \beta_{\mathrm{C} 18 \mathrm{~N} 3 \mathrm{C} 2}(7)$ \\
\hline 27 & 669.8 & 2.0 & 0.2 & ${ }^{3} \tau_{1 / R 6}(37), \omega_{\mathrm{C} 9 \mathrm{O} 5}(13),{ }^{1} \tau_{1 / R 6}(12), \omega_{\mathrm{C} 18 \mathrm{~N} 3}(11)$ \\
\hline 28 & 672.8 & 13.9 & 1.6 & ${ }^{3} \beta_{3 / R \sigma}(38),{ }^{3} \beta_{2 / R \sigma}(10), v_{\mathrm{C} 10 \mathrm{C} 11}(7)$ \\
\hline 29 & 690.4 & 57.0 & 0.1 & $\tau_{\mathrm{N} 3 \mathrm{C} 18}(26), \omega_{\mathrm{N} 3 \mathrm{H} 23}(20), \tau_{\mathrm{C} 2 \mathrm{~N} 3}(12),{ }^{1} \tau_{1 / R 6}(10)$ \\
\hline 30 & 705.4 & 7.4 & 1.6 & $\rho_{\mathrm{C} 9 \mathrm{O} 5}(19),{ }^{3} \beta_{2 / R 6}(17), \rho_{\mathrm{C} 17 \mathrm{O} 4}(17),{ }^{1} \beta_{2 / R 6}(10),{ }^{1} \beta_{3 / R 6}(8)$ \\
\hline 31 & 717.3 & 4.5 & 1.0 & $\begin{array}{l}\rho_{\mathrm{C}_{2} 0 \mathrm{H} 31 \mathrm{H} 32}(32), v_{\mathrm{S}_{1} \mathrm{C} 20}(23), \beta_{\mathrm{C}_{20}{ }_{1} \mathrm{H}_{33}}(18), \rho_{\mathrm{C}_{2} \mathrm{H} 22 \mathrm{H} 21}(7) \\
\delta_{2 / \mathrm{C} 2 \mathrm{H} 22 \mathrm{H} 21}(6)\end{array}$ \\
\hline 32 & 726.0 & 31.3 & 0.0 & ${ }^{3} \tau_{1 / R 6}(28),{ }^{1} \tau_{1 / R 6}(26), \omega_{\mathrm{C} 6 \mathrm{H} 24}(8), \omega_{\mathrm{C}_{15 \mathrm{H} 29}}(8), \omega_{\mathrm{C} 17 \mathrm{O} 4}(7)$ \\
\hline 33 & 748.2 & 65.3 & 0.0 & $\begin{array}{l}{ }_{\tau_{1 / R 6}}(15), \omega_{\mathrm{C} 18 \mathrm{~N} 3}(14), \omega_{\mathrm{C} 13 \mathrm{H} 27}(13), \omega_{\mathrm{C} 9 \mathrm{O} 5}(8),{ }^{3} \tau_{1 / R 6}(8), \\
\omega_{\mathrm{C} 17 \mathrm{O} 4}(8), \omega_{\mathrm{C} 14 \mathrm{H} 28}(7), \omega_{\mathrm{C} 15 \mathrm{H} 29}(6)\end{array}$ \\
\hline 34 & 760.9 & 3.0 & 3.2 & $\begin{array}{l}v_{\mathrm{S}_{1} \mathrm{C}_{20}}(32), \rho_{\mathrm{C} 20 \mathrm{H} 31 \mathrm{H} 32}(25), \delta_{2 / \mathrm{C}_{2} \mathrm{H} 22 \mathrm{H} 21}(9), \rho_{\mathrm{C}_{2} \mathrm{H} 22 \mathrm{H} 21}(8), \\
\delta_{2 / \mathrm{C} 20 \mathrm{H} 31 \mathrm{H} 32}(5)\end{array}$ \\
\hline
\end{tabular}




\begin{tabular}{|c|c|c|c|c|}
\hline 35 & 788.6 & 5.2 & 0.0 & $\begin{array}{l}{ }^{3} \tau_{1 / R 6}(18),{ }^{2} \tau_{1 / R 6}(13), \omega_{\mathrm{C} 7 \mathrm{H} 25}(13), \omega_{\mathrm{C} 19 \mathrm{H} 30}(11), \omega_{\mathrm{C} 6 \mathrm{H} 24}(11), \\
\omega_{\mathrm{C} 17 \mathrm{O} 4}(10), \omega_{\mathrm{C} 9 \mathrm{O} 5}(9), \omega_{\mathrm{C}_{15} \mathrm{H}_{29}}(5)\end{array}$ \\
\hline 36 & 792.2 & 1.3 & 1.1 & ${ }^{1} \beta_{\text {trig/R6 }}(18),{ }_{3 / R 6}^{1} \beta_{3 / 2},{ }^{3} \beta_{2 / R 6}(13),{ }^{3} \beta_{\text {trig/R6 }}(8), v_{\mathrm{C} 9 \mathrm{C} 10}(7)$ \\
\hline 37 & 810.1 & 0.9 & 0.1 & $\begin{array}{l}\omega_{\mathrm{C} 9 \mathrm{O} 5}(27), \omega_{\mathrm{C} 13 \mathrm{H} 27}(14),{ }^{1} \tau_{1 / R 6}(11), \omega_{\mathrm{C} 18 \mathrm{~N} 3}(8),{ }^{2} \tau_{1 / R 6}(8), \\
\omega_{\mathrm{C} 15 \mathrm{H} 29}(7)\end{array}$ \\
\hline 38 & 827.6 & 26.3 & 0.0 & $\begin{array}{l}\omega_{\mathrm{C} 7 \mathrm{H} 25}(23), \omega_{\mathrm{C}_{17} \mathrm{O} 4}(17),{ }^{1} \tau_{1 / R 6}(16), \omega_{\mathrm{C} 18 \mathrm{~N} 3}(9),{ }^{2} \tau_{1 / R 6}(7), \\
\omega_{\mathrm{C} 6 \mathrm{H} 24}(7), \omega_{\mathrm{C} 15 \mathrm{H} 29}(6)\end{array}$ \\
\hline 39 & 855.6 & 4.9 & 1.2 & $\beta_{\mathrm{C}_{20 \mathrm{~S} 1 \mathrm{H} 33}}(24), \rho_{\mathrm{C}_{2} \mathrm{H} 22 \mathrm{H} 21}(14), \tau_{\mathrm{C}_{20} \mathrm{H} 31 \mathrm{H} 32}(9),{ }^{1} \beta_{2 / R 6}(6),{ }^{2} \beta_{\text {trig/R6}}(5)$ \\
\hline 40 & 888.5 & 22.7 & 1.7 & $\beta_{\mathrm{C}_{20 \mathrm{~S} 1 \mathrm{H} 33}}(24), \tau_{\mathrm{C}_{20 \mathrm{H} 31 \mathrm{H} 32}}(10), \rho_{\mathrm{C}_{2} \mathrm{H} 22 \mathrm{H} 21}(9), v_{\mathrm{C} 7 \mathrm{C} 18}(6), \rho_{\mathrm{C} 17 \mathrm{O} 4}(6)$ \\
\hline 41 & 913.1 & 0.4 & 0.0 & $\begin{array}{l}\omega_{\mathrm{C} 19 \mathrm{H} 30}(22), \omega_{\mathrm{C} 12 \mathrm{H} 26}(18), \omega_{\mathrm{C} 14 \mathrm{H} 28}(17), \omega_{\mathrm{C} 7 \mathrm{H} 25}(16), \omega_{\mathrm{C} 15 \mathrm{H} 29}(9), \\
\omega_{\mathrm{C} 13 \mathrm{H} 27}(7)\end{array}$ \\
\hline 42 & 919.7 & 0.8 & 0.0 & $\omega_{\mathrm{C} 19 \mathrm{H} 30}(28), \omega_{\mathrm{C} 7 \mathrm{H} 25}(18), \omega_{\mathrm{C} 14 \mathrm{H} 28}(13), \omega_{\mathrm{C} 12 \mathrm{H} 26}(11), \omega_{\mathrm{C} 13 \mathrm{H} 27}(7)$ \\
\hline 43 & 928.9 & 2.6 & 1.3 & ${ }^{1} \beta_{\text {trig/R6}}(25),{ }^{3} \beta_{\text {trig/R6 }}(24), \rho_{\mathrm{C} 9 \mathrm{O} 5}(14), v_{\mathrm{C} 16 \mathrm{C} 18}(7), \rho_{\mathrm{C} 17 \mathrm{O} 4}(5)$ \\
\hline 44 & 999.6 & 87.6 & 2.0 & $v_{\mathrm{C}_{2} \mathrm{~N} 3}(15), v_{\mathrm{C} 8 \mathrm{C} 17}(12), v_{\mathrm{C}_{11} \mathrm{C}_{12}}(8), \rho_{\mathrm{C} 17 \mathrm{O} 4}(7), v_{\mathrm{C} 8 \mathrm{C} 19}(6), \rho_{\mathrm{C} 9 \mathrm{O} 5}(5)$ \\
\hline 45 & 1001.5 & 1.9 & 0.1 & $\begin{array}{l}\omega_{\mathrm{C} 6 \mathrm{H} 24}(43), \omega_{\mathrm{C} 19 \mathrm{H} 30}(18), \omega_{\mathrm{C} 7 \mathrm{H} 25}(9), \omega_{\mathrm{C}_{12} \mathrm{H} 26}(7), \omega_{\mathrm{C} 14 \mathrm{H} 28}(6), \\
{ }^{1} \tau_{1 / R 6}(6)\end{array}$ \\
\hline 46 & 1003.5 & 2.3 & 0.0 & $\begin{array}{l}\omega_{\mathrm{C} 12 \mathrm{H} 26}(27), \omega_{\mathrm{C} 14 \mathrm{H} 28}(25), \omega_{\mathrm{C} 6 \mathrm{H} 24}(12), \omega_{\mathrm{C} 13 \mathrm{H} 27}(9), \omega_{\mathrm{C} 15 \mathrm{H} 29}(8), \\
\omega_{\mathrm{C} 19 \mathrm{H} 30}(6)\end{array}$ \\
\hline 47 & 1016.8 & 6.1 & 0.9 & $v_{\mathrm{C}_{2} \mathrm{C} 20}(72), \delta_{2 / \mathrm{C}_{2} \mathrm{H} 22 \mathrm{H} 21}(6)$ \\
\hline 48 & 1017.0 & 0.1 & 0.0 & $\omega_{\mathrm{C} 15 \mathrm{H} 29}(29), \omega_{\mathrm{C} 13 \mathrm{H} 27}(28), \omega_{\mathrm{C} 14 \mathrm{H} 28}(15), \omega_{\mathrm{C} 12 \mathrm{H} 26}(13),{ }^{3} \tau_{1 / R 6}(12)$ \\
\hline 49 & 1056.7 & 1.7 & 5.9 & $v_{\mathrm{C} 13 \mathrm{C} 15}(35), v_{\mathrm{C} 14 \mathrm{C} 15}(13), v_{\mathrm{C} 12 \mathrm{C} 13}(11), \rho_{\mathrm{C} 12 \mathrm{H} 26}(11), \rho_{\mathrm{C} 14 \mathrm{H} 28}(11)$ \\
\hline 50 & 1082.8 & 30.9 & 0.3 & $\rho_{\mathrm{C}_{2} \mathrm{H}_{22} \mathrm{H} 21}(22), \rho_{\mathrm{C}_{20} \mathrm{H}_{31 \mathrm{H} 32}}(18), \beta_{{\mathrm{C} 20 \mathrm{~S}_{1} \mathrm{H}_{33}}}(17), v_{\mathrm{C} 6 \mathrm{C} 19}(7), v_{\mathrm{C} 7 \mathrm{C} 18}(6)$ \\
\hline 51 & 1099.9 & 17.1 & 0.9 & $v_{\mathrm{C} 6 \mathrm{C} 19}(23),{ }^{1} \beta_{t r i g / R 6}(16), \rho_{\mathrm{C} 19 \mathrm{H} 30}(13), v_{\mathrm{C}_{2 N} 3}(12)$ \\
\hline 52 & 1113.1 & 1.3 & 0.4 & $\begin{array}{l}{ }^{3} \beta_{\text {trig/R6 }}(38), v_{\mathrm{C} 12 \mathrm{C} 13}(8), v_{\mathrm{C} 14 \mathrm{C} 15}(8), \rho_{\mathrm{C} 13 \mathrm{H} 27}(8), \rho_{\mathrm{C} 15 \mathrm{H} 29}(7), \\
v_{\mathrm{C} 9 \mathrm{C} 10}(6), \rho_{\mathrm{C} 14 \mathrm{H} 28}(5), v_{\mathrm{C} 11 \mathrm{C} 17}(5)\end{array}$ \\
\hline 53 & 1129.7 & 8.8 & 3.0 & $v_{\mathrm{C}_{2 \mathrm{~N}}(}(37), \rho_{\mathrm{C} 18 \mathrm{~N} 3}(8), \tau_{\mathrm{C}_{20 \mathrm{H} 31 \mathrm{H} 32}}(6), v_{\mathrm{C} 8 \mathrm{C} 17}(6)$ \\
\hline 54 & 1175.8 & 7.8 & 4.4 & $\begin{array}{l}\rho_{\mathrm{C} 13 \mathrm{H} 27}(16), \rho_{\mathrm{C} 12 \mathrm{H} 26}(12), v_{\mathrm{C} 9 \mathrm{C} 16}(10),{ }^{1} \beta_{\text {trig/R6 }}(7), v_{\mathrm{C} 9 \mathrm{C} 10}(7), \\
{ }^{3} \beta_{\text {trig/R6}}(6)\end{array}$ \\
\hline 55 & 1183.1 & 15.6 & 0.2 & $\rho_{\mathrm{C} 15 \mathrm{H} 29}(31), \rho_{\mathrm{C} 14 \mathrm{H} 28}(11), \rho_{\mathrm{C} 13 \mathrm{H} 27}(9), v_{\mathrm{C} 14 \mathrm{C} 15}(8), v_{\mathrm{C} 13 \mathrm{C} 15}(6)$ \\
\hline 56 & 1197.9 & 31.3 & 10.1 & $\rho_{\mathrm{C} 6 \mathrm{H} 24}(26), \rho_{\mathrm{C} 7 \mathrm{H} 25}(11), \rho_{\mathrm{C} 13 \mathrm{H} 27}(7), v_{\mathrm{C} 6 \mathrm{C} 7}(7)$ \\
\hline 57 & 1214.3 & 5.2 & 4.2 & $\rho_{\mathrm{C} 19 \mathrm{H} 30}(21), \rho_{\mathrm{C} 7 \mathrm{H} 25}(17), \rho_{\mathrm{C} 6 \mathrm{H} 24}(9), v_{\mathrm{C} 8 \mathrm{C} 19}(8), \tau_{\mathrm{C} 20 \mathrm{H} 31 \mathrm{H} 32}(6)$ \\
\hline 58 & 1222.3 & 29.7 & 0.3 & $\tau_{\mathrm{C} 20 \mathrm{H} 31 \mathrm{H} 32}(28), \tau_{\mathrm{C} 2 \mathrm{H} 22 \mathrm{H} 21}(12), \rho_{\mathrm{C} 2 \mathrm{H} 22 \mathrm{H} 21}(7)$ \\
\hline 59 & 1264.6 & 52.1 & 2.6 & $\begin{array}{l}\rho_{\mathrm{C} 12 \mathrm{H} 26}(16),{ }^{2} \beta_{\text {trig/R6 }}(9), \rho_{\mathrm{C} 14 \mathrm{H} 28}(9), v_{\mathrm{C}_{11 \mathrm{C} 12}}(7), \tau_{\mathrm{C} 20 \mathrm{H} 31 \mathrm{H} 32}(5), \\
v_{\mathrm{C} 7 \mathrm{C} 18}(5)\end{array}$ \\
\hline 60 & 1279.5 & 92.1 & 0.4 & $\omega_{\mathrm{C} 20 \mathrm{H} 31 \mathrm{H} 32}(69), \omega_{\mathrm{C} 2 \mathrm{H} 22 \mathrm{H} 21}(11)$ \\
\hline 61 & 1289.8 & 580.8 & 8.2 & $\begin{array}{l}v_{\mathrm{C} 9 \mathrm{C} 10}(12), v_{\mathrm{C} 9 \mathrm{C} 16}(8), v_{\mathrm{C} 8 \mathrm{C} 16}(8), \rho_{\mathrm{C} 14 \mathrm{H} 28}(7), v_{\mathrm{C} 7 \mathrm{C} 18}(6), v_{\mathrm{C} 8 \mathrm{C} 17}(6), \\
\rho_{\mathrm{C} 19 \mathrm{H} 30}(6), v_{\mathrm{C} 6 \mathrm{C} 19}(5)\end{array}$ \\
\hline 62 & 1316.5 & 119.1 & 1.5 & $v_{\mathrm{C} 11 \mathrm{C} 17}(19), v_{\mathrm{C} 8 \mathrm{C} 17}(13), \rho_{\mathrm{C} 12 \mathrm{H} 26}(9), \rho_{\mathrm{C} 17 \mathrm{O} 4}(7),{ }^{2} \beta_{2 / R 6}(6)$ \\
\hline 63 & 1327.4 & 72.8 & 6.5 & $\tau_{\mathrm{C} 2 \mathrm{H} 22 \mathrm{H} 21}(33), v_{\mathrm{N} 3 \mathrm{C} 18}(14),{ }^{1} \beta_{\text {trig/R6}}(8), \tau_{\mathrm{C}_{20 \mathrm{H} 31 \mathrm{H} 32}}(7), v_{\mathrm{C}_{16 \mathrm{C} 18}}(6)$ \\
\hline 64 & 1336.8 & 124.5 & 2.8 & $\begin{array}{l}\tau_{\mathrm{C} 2 \mathrm{H} 22 \mathrm{H} 21}(16), v_{\mathrm{N} 3 \mathrm{C} 18}(14), \rho_{\mathrm{N} 3 \mathrm{H} 23}(11), v_{\mathrm{C} 16 \mathrm{C} 18}(9), v_{\mathrm{C} 9 \mathrm{C} 16}(7), \\
\rho_{\mathrm{C} 6 \mathrm{H} 24}(6), \omega_{\mathrm{C} 2 \mathrm{H} 22 \mathrm{H} 21}(6)\end{array}$ \\
\hline 65 & 1358.6 & 15.0 & 1.0 & $\begin{array}{l}v_{\mathrm{C} 10 \mathrm{C} 11}(15), v_{\mathrm{C} 10 \mathrm{C} 14}(15), v_{\mathrm{C} 11 \mathrm{C} 12}(14), v_{\mathrm{C} 13 \mathrm{C} 15}(14), v_{\mathrm{C} 12 \mathrm{C} 13}(13), \\
v_{\mathrm{C} 14 \mathrm{C} 15}(13)\end{array}$ \\
\hline 66 & 1378.4 & 24.4 & 1.4 & $\begin{array}{l}v_{\mathrm{C} 16 \mathrm{C} 18}(18), v_{\mathrm{C} 9 \mathrm{C} 16}(10), v_{\mathrm{C} 8 \mathrm{C} 19}(10), v_{\mathrm{C} 8 \mathrm{C} 16}(8), v_{\mathrm{C} 6 \mathrm{C} 19}(7), \rho_{\mathrm{C} 9 \mathrm{O} 5}(6), \\
v_{\mathrm{C} 6 \mathrm{C} 7}(6), v_{\mathrm{C} 7 \mathrm{C} 18}(5), v_{\mathrm{C} 9 \mathrm{C} 10}(5)\end{array}$ \\
\hline 67 & 1388.5 & 15.7 & 2.1 & $\omega_{\mathrm{C}_{2} \mathrm{H} 22 \mathrm{H} 21}(62), \omega_{\mathrm{C} 20 \mathrm{H} 31 \mathrm{H} 32}(6), v_{\mathrm{C} 8 \mathrm{C} 16}(6)$ \\
\hline 68 & 1435.3 & 30.9 & 0.5 & $\rho_{\mathrm{C} 7 \mathrm{H} 25}(25), v_{\mathrm{C} 6 \mathrm{C} 7}(11), v_{\mathrm{C} 8 \mathrm{C} 19}(11), v_{\mathrm{C} 8 \mathrm{C} 16}(6), v_{\mathrm{C} 9 \mathrm{C} 16}(6)$ \\
\hline 69 & 1476.2 & 3.6 & 4.5 & $\delta_{1 / \mathrm{C}_{20} \mathrm{H} 31 \mathrm{H} 32}(69), \delta_{1 / \mathrm{C}_{2} \mathrm{H}_{22} \mathrm{H}_{21}}(5)$ \\
\hline 70 & 1479.2 & 1.6 & 2.0 & $\delta_{1 / \mathrm{C} 20 \mathrm{H} 31 \mathrm{H} 32}(18), \rho_{\mathrm{C}_{15} \mathrm{H} 29}(14), \rho_{\mathrm{C} 13 \mathrm{H} 27}(12), v_{\mathrm{C} 10 \mathrm{C} 14}(6), v_{\mathrm{C} 11 \mathrm{C} 12}(6)$ \\
\hline
\end{tabular}




\begin{tabular}{|c|c|c|c|c|}
\hline 71 & 1488.4 & 10.0 & 0.9 & $\begin{array}{l}\rho_{\mathrm{C} 19 \mathrm{H} 30}(12), v_{\mathrm{C} 8 \mathrm{C} 16}(9), \rho_{\mathrm{C} 6 \mathrm{H} 24}(7), \rho_{\mathrm{C} 13 \mathrm{H} 27}(6), v_{\mathrm{C} 7 \mathrm{C} 18}(5), v_{\mathrm{C} 8 \mathrm{C} 17}(5), \\
\rho_{\mathrm{C} 15 \mathrm{H} 29}(5)\end{array}$ \\
\hline 72 & 1507.4 & 2.6 & 1.9 & $\delta_{1 / \mathrm{C} 2 \mathrm{H} 22 \mathrm{H} 21}(17), \rho_{\mathrm{C} 14 \mathrm{H} 28}(16), \rho_{\mathrm{C} 12 \mathrm{H} 26}(16), v_{\mathrm{C} 10 \mathrm{C} 11}(14), v_{\mathrm{C} 13 \mathrm{C} 15}(7)$ \\
\hline 73 & 1510.1 & 6.1 & 0.9 & $\delta_{1 / \mathrm{C}_{2} \mathrm{H}_{22 \mathrm{H} 21}(67)}$ \\
\hline 74 & 1552.8 & 202.1 & 2.0 & $v_{\mathrm{C} 6 \mathrm{C} 19}(19), \rho_{\mathrm{N} 3 \mathrm{H} 23}(18), v_{\mathrm{N} 3 \mathrm{C} 18}(16), \rho_{\mathrm{C} 6 \mathrm{H} 24}(12), v_{\mathrm{C} 6 \mathrm{C} 7}(7), v_{\mathrm{C} 8 \mathrm{C} 16}(6)$ \\
\hline 75 & 1611.1 & 35.8 & 11.7 & $\begin{array}{l}v_{\mathrm{C} 13 \mathrm{C} 15}(13), v_{\mathrm{C} 10 \mathrm{C} 11}(13), v_{\mathrm{O} 5 \mathrm{C} 9}(10), v_{\mathrm{C}_{12} \mathrm{C} 13}(7),{ }^{3} \beta_{3 / R 6}(6), v_{\mathrm{C} 8 \mathrm{C} 19}(5), \\
v_{\mathrm{C} 10 \mathrm{C} 14}(5)\end{array}$ \\
\hline 76 & 1614.3 & 92.0 & 7.5 & $\begin{array}{l}\rho_{\mathrm{N}_{3} \mathrm{H} 23}(21), v_{\mathrm{C} 6 \mathrm{C} 19}(9), v_{\mathrm{C} 8 \mathrm{C} 19}(8), v_{\mathrm{C} 16 \mathrm{C} 18}(8), v_{\mathrm{C} 7 \mathrm{C} 18}(7), v_{\mathrm{C} 13 \mathrm{C} 15}(6), \\
v_{\mathrm{C} 10 \mathrm{C} 11}(6),{ }^{1} \beta_{2 / R 6}(5)\end{array}$ \\
\hline 77 & 1626.7 & 42.2 & 16.9 & $\begin{array}{l}v_{\mathrm{C} 14 \mathrm{C} 15}(14), v_{\mathrm{C} 11 \mathrm{C} 12}(11), v_{\mathrm{O} 5 \mathrm{C} 9}(8),{ }^{3} \beta_{2 / R 6}(7), v_{\mathrm{C} 6 \mathrm{C} 7}(6), v_{\mathrm{C} 12 \mathrm{C} 13}(6), \\
\rho_{\mathrm{N} 3 \mathrm{H} 23}(5)\end{array}$ \\
\hline 78 & 1630.8 & 285.0 & 5.3 & $\begin{array}{l}v_{\mathrm{C} 6 \mathrm{C} 7}(15), v_{\mathrm{C}_{14} \mathrm{C} 15}(7), v_{\mathrm{C} 8 \mathrm{C} 19}(6), v_{\mathrm{O} 4 \mathrm{C} 17}(6), v_{\mathrm{C} 12 \mathrm{C} 13}(6),{ }^{1} \beta_{3 / R 6}(6), \\
\rho_{\mathrm{C} 7 \mathrm{H} 25}(5)\end{array}$ \\
\hline 79 & 1668.6 & 209.3 & 31.2 & $v_{\mathrm{O} 5 \mathrm{C} 9}(42),{ }^{2} \beta_{3 / R \sigma}(6),{ }^{2} \beta_{\text {trig/R6}}(6)$ \\
\hline 80 & 1721.0 & 180.2 & 32.1 & $v_{\mathrm{O} 4 \mathrm{C} 17}(71),{ }^{2} \beta_{\text {trig/R6}}(9)$ \\
\hline 81 & 2661.6 & 5.0 & 10.3 & $v_{\mathrm{S}_{1 H} 33}(100)$ \\
\hline 82 & 3046.0 & 29.3 & 9.3 & $v_{\mathrm{C} 2 \mathrm{H} 21}(52), v_{\mathrm{C}_{2} \mathrm{H} 22}(43)$ \\
\hline 83 & 3068.8 & 20.7 & 1.9 & $v_{\mathrm{C} 20 \mathrm{H} 31}(51), v_{\mathrm{C}_{20} \mathrm{H} 32}(44)$ \\
\hline 84 & 3082.3 & 6.5 & 4.0 & $v_{\mathrm{C} 2 \mathrm{H} 22}(47), v_{\mathrm{C} 2 \mathrm{H} 21}(41), v_{\mathrm{C}_{20 \mathrm{H} 32}}(7)$ \\
\hline 85 & 3119.5 & 8.6 & 0.7 & $v_{\mathrm{C} 20 \mathrm{H} 32}(47), v_{\mathrm{C}_{20} \mathrm{H} 31}(42), v_{\mathrm{C}_{2} \mathrm{H}_{22}}(6)$ \\
\hline 86 & 3170.1 & 3.4 & 4.3 & $v_{\mathrm{C} 15 \mathrm{H} 29}(49), v_{\mathrm{C} 13 \mathrm{H} 27_{2}}(45)$ \\
\hline 87 & 3173.0 & 10.1 & 7.1 & $v_{\mathrm{C} 6 \mathrm{H} 24}(89), v_{\mathrm{C} 7 \mathrm{H} 25}(7)$ \\
\hline 88 & 3184.9 & 13.6 & 14.3 & $v_{\mathrm{C} 13 \mathrm{H} 27}(43), v_{\mathrm{C} 15 \mathrm{H} 29}(42), v_{\mathrm{C} 12 \mathrm{H} 26}(9), v_{\mathrm{C} 14 \mathrm{H} 28}(5)$ \\
\hline 89 & 3201.8 & 3.6 & 6.5 & $v_{\mathrm{C} 12 \mathrm{H} 26}(81), v_{\mathrm{C} 14 \mathrm{H} 28}(9), v_{\mathrm{C} 13 \mathrm{H} 27}(9)$ \\
\hline 90 & 3204.3 & 10.6 & 7.9 & $v_{\mathrm{C} 7 \mathrm{H} 25}(89)$ \\
\hline 91 & 3205.9 & 9.0 & 12.6 & $v_{\mathrm{C} 14 \mathrm{H} 28}(82), v_{\mathrm{C} 15 \mathrm{H} 29}(8), v_{\mathrm{C} 12 \mathrm{H} 26}(7)$ \\
\hline 92 & 3212.6 & 4.0 & 12.2 & $v_{\mathrm{C} 19 \mathrm{H} 30}(92)$ \\
\hline 93 & 3460.1 & 115.4 & 36.4 & $v_{\mathrm{N} 3 \mathrm{H} 23}(99)$ \\
\hline
\end{tabular}


Table S.7. Frequencies of normal vibrations, IR and Raman intensities and PEDs for Ac-GT conformer calculated at the B3LYP/6-311++G(d,p) level of theory.

\begin{tabular}{|c|c|c|c|c|}
\hline $\mathbf{N r}$ & $\begin{array}{l}\text { Freq. } \\
{\left[\mathrm{cm}^{-1}\right]}\end{array}$ & IR Int. & $\begin{array}{c}\text { Raman } \\
\text { Int. }\end{array}$ & PED (contributions $>5 \%$ ) \\
\hline 1 & 21.1 & 1.0 & 12.3 & $\tau_{\mathrm{N} 3 \mathrm{C} 18}(45), \omega_{\mathrm{N} 3 \mathrm{H} 23}(35),{ }^{2} \tau_{3 / R 6}(7)$ \\
\hline 2 & 29.6 & 0.3 & 6.2 & $\tau_{\mathrm{C}_{2 \mathrm{~N} 3}}(40), \tau_{\mathrm{N} 3 \mathrm{C} 18}(19), \omega_{\mathrm{N} 3 \mathrm{H} 23}(11),{ }^{1} \tau_{3 / R 6}(8),{ }^{2} \tau_{3 / R 6}(6)$ \\
\hline 3 & 46.3 & 5.1 & 1.2 & ${ }^{2} \tau_{3 / R \sigma}(40), \tau_{\mathrm{C} 2 \mathrm{~N} 3}(17),{ }^{2} \tau_{2 / R \sigma}(16)$ \\
\hline 4 & 69.7 & 0.6 & 0.6 & ${ }^{2} \tau_{2 / R 6}(21), \tau_{\mathrm{C}_{2} \mathrm{C} 20}(16),{ }^{1} \tau_{3 / R 6}(15), \omega_{\mathrm{N} 3 \mathrm{H} 23}(9),{ }^{2} \tau_{3 / R 6}(6),{ }^{1} \tau_{2 / R 6}(5)$ \\
\hline 5 & 93.0 & 7.8 & 0.6 & $\tau_{\mathrm{C}_{2} \mathrm{C} 20}(30), \omega_{\mathrm{N} 3 \mathrm{H} 23}(20), \tau_{\mathrm{S} 1 \mathrm{C} 20}(13),{ }^{2} \tau_{2 / R 6}(11), \tau_{\mathrm{N} 3 \mathrm{C} 18}(7)$ \\
\hline 6 & 119.7 & 1.6 & 0.6 & ${ }^{2} \tau_{1 / R \sigma}(39),{ }^{3} \tau_{2 / R \sigma}(17),{ }^{2} \tau_{2 / R 6}(12),{ }^{3} \tau_{3 / R \sigma}(8)$ \\
\hline 7 & 144.1 & 8.1 & 1.1 & $\tau_{\mathrm{S} 1 \mathrm{C} 20}(64), \rho_{\mathrm{C} 18 \mathrm{~N} 3}(9), \beta_{\mathrm{C} 18 \mathrm{~N} 3 \mathrm{C} 2}(8)$ \\
\hline 8 & 154.1 & 5.5 & 0.7 & $\delta_{2 / \mathrm{C} 20 \mathrm{H} 31 \mathrm{H} 32}(22), \tau_{\mathrm{S}_{1 C} 20}(16), \omega_{\mathrm{N} 3 \mathrm{H} 23}(13),{ }^{2} \tau_{1 / R 6}(8), \rho_{\mathrm{C} 18 \mathrm{~N} 3}(6)$ \\
\hline 9 & 166.7 & 0.4 & 0.0 & $\begin{array}{l}{ }^{4} b f l_{R R}(25),{ }^{2} b f l_{R R}(22), \tau_{N 3 C l 8}(10),{ }^{2} \tau_{3 / R 6}(8), \omega_{C 17 O 4}(6), \omega_{C 9 O 5}(6), \\
{ }^{1} \tau_{3 / R 6}(5)\end{array}$ \\
\hline 10 & 195.9 & 0.3 & 1.2 & ${ }^{1}{ }^{1} \tau_{2 / R 6}(26),{ }^{1} \tau_{3 / R 6}(25),{ }^{4} b f l_{R R}(13), \omega_{C 19 H 30}(7)$ \\
\hline 11 & 234.9 & 1.1 & 0.2 & $\delta_{2 / \mathrm{C}_{20 \mathrm{H} 31 \mathrm{H} 32}}(24), \delta_{2 / \mathrm{C} 2 \mathrm{H} 22 \mathrm{H} 21}(11),{ }^{3} \beta_{2 / R 6}(7)$ \\
\hline 12 & 257.8 & 0.4 & 0.7 & ${ }^{2} b f l_{R R}(28), \omega_{C_{18 N 3}}(11),{ }^{4} b f l_{R R}(7), \delta_{2 / C_{2} 0 H_{31 H} 32}(6)$ \\
\hline 13 & 296.2 & 4.6 & 0.5 & ${ }^{2} \beta_{2 / R 6}(30), \delta_{2 / \mathrm{C}_{20 \mathrm{H} 31 \mathrm{H} 32}}(18), \rho_{\mathrm{C} 18 \mathrm{~N} 3}(6), v_{\mathrm{C} 8 \mathrm{C} 17}(6)$ \\
\hline 14 & 321.7 & 0.4 & 0.6 & $\beta_{\mathrm{C}_{18 \mathrm{~N} 3 \mathrm{C} 2}}(16), \delta_{2 / \mathrm{C}_{2} \mathrm{H}_{22} \mathrm{H}_{21}}(15), \tau_{\mathrm{C}_{2} \mathrm{C}_{20}}(8), \tau_{\mathrm{C}_{2} \mathrm{~N} 3}(7), \delta_{2 / \mathrm{C}_{20 \mathrm{H} 31 \mathrm{H} 32}}(5)$ \\
\hline 15 & 349.8 & 1.4 & 0.0 & ${ }^{2} \beta_{3 / R 6}(25), \rho_{\mathrm{C} 18 \mathrm{~N} 3}(15), \rho_{\mathrm{C} 17 \mathrm{O} 4}(13),{ }^{2} \beta_{2 / R 6}(9)$ \\
\hline 16 & 368.8 & 10.7 & 1.1 & ${ }^{2} \beta_{3 / R 6}(17),{ }^{1} \beta_{3 / R 6}(12), v_{\mathrm{C} 11 \mathrm{C} 17}(9), \rho_{\mathrm{C} 17 \mathrm{O} 4}(9), v_{\mathrm{C} 9 \mathrm{C} 16}(8), \rho_{\mathrm{C} 18 \mathrm{~N} 3}(7)$ \\
\hline 17 & 426.2 & 0.1 & 0.0 & ${ }^{3} \tau_{3 / R 6}(57),{ }^{4} b f l_{R R}(14),{ }^{3} \tau_{2 / R 6}(7)$ \\
\hline 18 & 429.7 & 21.7 & 0.5 & $\rho_{\mathrm{C} 9 \mathrm{O} 5}(40), \rho_{\mathrm{C} 17 \mathrm{O} 4}(16), v_{\mathrm{C} 9 \mathrm{C} 16}(9),{ }^{1} \beta_{3 / R 6}(9)$ \\
\hline 19 & 432.8 & 0.2 & 0.1 & ${ }^{3} \tau_{2 / R \sigma}(30),{ }^{1} \tau_{2 / R \sigma}(21),{ }^{1} \tau_{3 / R 6}(17),{ }^{2} \tau_{1 / R 6}(7), \omega_{\mathrm{C} 9 \mathrm{O} 5}(6)$ \\
\hline 20 & 441.0 & 2.4 & 0.2 & $\begin{array}{l}\delta_{2 / \mathrm{C}_{2} \mathrm{H}_{22} \mathrm{H}_{21}}(17),{ }^{2} \beta_{2 / R \sigma}(14),{ }^{1} \beta_{2 / R \sigma}(12),{ }^{2} \beta_{3 / R 6}(8),{ }^{3} \beta_{2 / R \sigma}(6), \\
\delta_{2 / \mathrm{C}_{20} \mathrm{H} 31 \mathrm{H} 32}(6)\end{array}$ \\
\hline 21 & 477.2 & 2.8 & 9.4 & ${ }^{2} \beta_{2 / R 6}(26),{ }^{2} \beta_{3 / R 6}(24),{ }^{1} \beta_{3 / R 6}(9),{ }^{3} \beta_{3 / R 6}(9), v_{\mathrm{C} 8 \mathrm{C} 16}(6), v_{\mathrm{C} 10 \mathrm{C} 11}(5)$ \\
\hline 22 & 489.5 & 1.0 & 0.1 & ${ }^{3} \tau_{2 / R \sigma}(23),{ }^{1} \tau_{3 / R 6}(22),{ }^{1} \tau_{2 / R 6}(11),{ }^{2} \tau_{2 / R \sigma}(9),{ }^{3} \tau_{3 / R 6}(6),{ }^{1} \tau_{1 / R 6}(5)$ \\
\hline 23 & 503.8 & 4.6 & 1.1 & $\begin{array}{l}\delta_{2 / \mathrm{C} 2 \mathrm{H} 22 \mathrm{H} 21}(22),{ }^{1} \beta_{2 / R 6}(10), \rho_{\mathrm{C} 18 \mathrm{~N} 3}(9),{ }^{3} \beta_{2 / R 6}(6), \beta_{\mathrm{C}_{18 \mathrm{~N} 3 \mathrm{C} 2}}(6), \\
\rho_{\mathrm{C} 20 \mathrm{H} 31 \mathrm{H} 32}(5)\end{array}$ \\
\hline 24 & 552.7 & 6.4 & 0.1 & $\omega_{\mathrm{C} 18 \mathrm{~N} 3}(31),{ }^{1} \tau_{3 / R 6}(14),{ }^{1} \tau_{2 / R 6}(14),{ }^{1} \tau_{1 / R 6}(8),{ }^{2} b f l_{R R}(6)$ \\
\hline 25 & 598.7 & 4.1 & 0.6 & $\begin{array}{l}{ }^{1} \beta_{3 / R 6}(17), \beta_{\mathrm{C} 18 \mathrm{~N} 3 \mathrm{C} 2}(12),{ }^{3} \beta_{2 / R 6}(10),{ }^{1} \beta_{2 / R 6}(10), \rho_{\mathrm{C} 18 \mathrm{~N} 3}(9), \\
{ }^{3} \beta_{3 / R 6}(7)\end{array}$ \\
\hline 26 & 630.3 & 1.2 & 0.1 & ${ }^{2} \beta_{\text {trig/R6 }}(43),{ }^{1} \beta_{2 / R 6}(17), \rho_{\mathrm{C} 18 \mathrm{~N} 3}(6)$ \\
\hline 27 & 652.8 & 4.3 & 2.6 & $v_{\mathrm{S}_{1} \mathrm{C} 20}(62),{ }^{3} \beta_{3 / R 6}(8), \delta_{2 / \mathrm{C}_{20} \mathrm{H} 31 \mathrm{H} 32}(6)$ \\
\hline 28 & 669.5 & 1.0 & 0.0 & ${ }^{3} \tau_{1 / R 6}(38),{ }^{1} \tau_{1 / R 6}(19), \omega_{\mathrm{C} 9 \mathrm{O} 5}(12), \omega_{\mathrm{C} 18 \mathrm{~N} 3}(12)$ \\
\hline 29 & 677.3 & 9.9 & 1.1 & ${ }^{3} \beta_{3 / R 6}(38),{ }^{3} \beta_{2 / R 6}(9), v_{\mathrm{C} 10 \mathrm{C} 11}(7), v_{\mathrm{S} 1 \mathrm{C} 20}(6)$ \\
\hline 30 & 705.3 & 1.0 & 0.4 & $\rho_{\mathrm{C} 9 \mathrm{O} 5}(20),{ }^{3} \beta_{2 / R 6}(19), \rho_{\mathrm{C} 17 \mathrm{O} 4}(18),{ }^{1} \beta_{2 / R 6}(11),{ }^{1} \beta_{3 / R 6}(8),{ }^{3} \beta_{3 / R 6}(5)$ \\
\hline 31 & 712.4 & 60.7 & 0.2 & ${ }^{1} \tau_{1 / R 6}(23), \tau_{\mathrm{N} 3 \mathrm{C} 18}(21), \omega_{\mathrm{N} 3 \mathrm{H} 23}(19), \tau_{\mathrm{C}_{2} \mathrm{~N} 3}(10), \omega_{\mathrm{C} 6 \mathrm{H} 24}(8),{ }^{3} \tau_{1 / R 6}(5)$ \\
\hline 32 & 725.9 & 20.4 & 0.0 & $\begin{array}{l}{ }^{3} \tau_{1 / R 6}(25),{ }^{1} \tau_{1 / R 6}(18), \omega_{\mathrm{C} 15 \mathrm{H} 29}(9), \omega_{\mathrm{C} 17 \mathrm{O} 4}(9), \omega_{\mathrm{C} 9 \mathrm{O} 5}(6), \omega_{\mathrm{C} 6 \mathrm{H} 24}(6), \\
\omega_{\mathrm{C} 12 \mathrm{H} 26}(5)\end{array}$ \\
\hline 33 & 750.3 & 88.7 & 0.0 & $\begin{array}{l}\omega_{\mathrm{C} 18 \mathrm{~N} 3}(14),{ }^{1} \tau_{1 / R 6}(13), \omega_{\mathrm{C} 13 \mathrm{H} 27}(13),{ }^{3} \tau_{1 / R 6}(9), \omega_{\mathrm{C} 9 \mathrm{O} 5}(7), \\
\omega_{\mathrm{C} 17 \mathrm{O} 4}(7), \omega_{\mathrm{C} 14 \mathrm{H} 28}(6), \omega_{\mathrm{C} 15 \mathrm{H} 29}(5)\end{array}$ \\
\hline 34 & 787.6 & 5.3 & 0.1 & $\begin{array}{l}{ }^{3} \tau_{1 / R 6}(17), \omega_{\mathrm{C} 7 \mathrm{H} 25}(14),{ }^{2} \tau_{1 / R 6}(12), \omega_{\mathrm{C} 19 \mathrm{H} 30}(12), \omega_{\mathrm{C} 6 \mathrm{H} 24}(11), \\
\omega_{\mathrm{C}_{17} \mathrm{O} 4}(9), \omega_{\mathrm{C} 95}(8), \omega_{\mathrm{C} 15 \mathrm{H} 29}(6)\end{array}$ \\
\hline 35 & 791.3 & 2.1 & 1.3 & ${ }^{1} \beta_{\text {trig/R6 }}(19),{ }^{1} \beta_{3 / R \sigma}(17),{ }^{3} \beta_{2 / R \sigma}(13),{ }^{3} \beta_{\text {trig/R6 }}(8), v_{\mathrm{C} 9 \mathrm{C} 10}(7)$ \\
\hline 36 & 809.3 & 0.4 & 0.1 & $\begin{array}{l}\omega_{\mathrm{C} 9 \mathrm{O} 5}(28), \omega_{\mathrm{C} 13 \mathrm{H} 27}(13),{ }^{1} \tau_{1 / R 6}(11),{ }^{2} \tau_{1 / R 6}(9), \omega_{\mathrm{C} 18 \mathrm{~N} 3}(8), \\
\omega_{\mathrm{C} 15 \mathrm{H} 29}(6)\end{array}$ \\
\hline 37 & 817.2 & 9.8 & 0.8 & $\beta_{\mathrm{C}_{20 \mathrm{~S} 1 \mathrm{H} 33}}(25), \rho_{\mathrm{C} 20 \mathrm{H} 31 \mathrm{H} 32}(23), v_{\mathrm{C}_{2} \mathrm{C} 20}(10), \rho_{\mathrm{C} 2 \mathrm{H} 22 \mathrm{H} 21}(6)$ \\
\hline
\end{tabular}




\begin{tabular}{|c|c|c|c|c|}
\hline 38 & 828.9 & 19.7 & 0.1 & $\begin{array}{l}\omega_{\mathrm{C} 7 \mathrm{H} 25}(20), \omega_{\mathrm{C} 17 \mathrm{O} 4}(16),{ }^{\mathrm{I}} \tau_{1 / R 6}(13), \omega_{\mathrm{C} 6 \mathrm{H} 24}(7), \omega_{\mathrm{C} 18 \mathrm{~N} 3}(7), \\
{ }^{2} \tau_{1 / R 6}(6), \omega_{\mathrm{C}_{15} \mathrm{H} 29}(5)\end{array}$ \\
\hline 39 & 861.4 & 13.7 & 1.6 & $\begin{array}{l}\beta_{\mathrm{C}_{20 \mathrm{~S} 1 \mathrm{H} 33}}(9),{ }^{1} \beta_{2 / R 6}(9), v_{\mathrm{C} 16 \mathrm{C} 18}(7), v_{\mathrm{C} 2 \mathrm{~N} 3}(7),{ }^{2} \beta_{\text {trig/R6 }}(7), \\
\rho_{\mathrm{C} 2 \mathrm{H} 22 \mathrm{H} 21}(7), \rho_{\mathrm{C} 17 \mathrm{O} 4}(6), v_{\mathrm{N}_{3} \mathrm{C} 18}(6), v_{\mathrm{C}_{11 \mathrm{C} 17}}(5)\end{array}$ \\
\hline 40 & 912.9 & 0.3 & 0.0 & $\begin{array}{l}\omega_{\mathrm{C} 14 \mathrm{H} 28}(22), \omega_{\mathrm{C} 12 \mathrm{H} 26}(21), \omega_{\mathrm{C} 19 \mathrm{H} 30}(16), \omega_{\mathrm{C} 7 \mathrm{H} 25}(11), \omega_{\mathrm{C} 15 \mathrm{H} 29}(10), \\
\omega_{\mathrm{C} 13 \mathrm{H} 27}(9)\end{array}$ \\
\hline 41 & 920.2 & 0.9 & 0.1 & $\omega_{\mathrm{C} 19 \mathrm{H} 30}(34), \omega_{\mathrm{C} 7 \mathrm{H} 25}(21), \omega_{\mathrm{C} 14 \mathrm{H} 28}(9), \omega_{\mathrm{C} 12 \mathrm{H} 26}(8), \omega_{\mathrm{C} 13 \mathrm{H} 27}(5)$ \\
\hline 42 & 929.0 & 3.0 & 1.4 & ${ }^{1} \beta_{\text {trig/R6}}(23),{ }^{3} \beta_{\text {trig/R6 }}(22), \rho_{\mathrm{C} 9 \mathrm{O} 5}(15), v_{\mathrm{C} 16 \mathrm{C} 18}(8)$ \\
\hline 43 & 935.2 & 8.3 & 0.5 & $\beta_{\mathrm{C}_{20 \mathrm{~S} 1 \mathrm{H} 33}}(28), \rho_{\mathrm{C} 20 \mathrm{H} 31 \mathrm{H} 32}(20), \rho_{\mathrm{C} 17 \mathrm{O} 4}(7)$ \\
\hline 44 & 956.2 & 13.2 & 0.4 & $\begin{array}{l}v_{\mathrm{C}_{2} \mathrm{C} 20}(28), \rho_{\mathrm{C} 2 \mathrm{H} 22 \mathrm{H} 21}(17), \rho_{\mathrm{C} 20 \mathrm{H} 31 \mathrm{H} 32}(14), \delta_{2 / \mathrm{C} 2 \mathrm{H} 22 \mathrm{H} 21}(11), v_{\mathrm{S}_{1} \mathrm{C} 20}(8), \\
\beta_{\mathrm{C}_{20} \mathrm{~S} 1 \mathrm{H} 33}(6)\end{array}$ \\
\hline 45 & 1001.6 & 0.4 & 0.0 & $\begin{array}{l}\omega_{\mathrm{C} 6 \mathrm{H} 24}(24), \omega_{\mathrm{C} 12 \mathrm{H} 26}(21), \omega_{\mathrm{C} 14 \mathrm{H} 28}(18), \omega_{\mathrm{C} 19 \mathrm{H} 30}(9), \omega_{\mathrm{C} 13 \mathrm{H} 27}(7), \\
\omega_{\mathrm{C} 15 \mathrm{H} 29}(6), \omega_{\mathrm{C} 7 \mathrm{H} 25}(6)\end{array}$ \\
\hline 46 & 1003.3 & 2.2 & 0.0 & $\omega_{\mathrm{C} 6 \mathrm{H} 24}(32), \omega_{\mathrm{C} 19 \mathrm{H} 30}(14), \omega_{\mathrm{C} 12 \mathrm{H} 26}(14), \omega_{\mathrm{C} 14 \mathrm{H} 28}(13), \omega_{\mathrm{C} 7 \mathrm{H} 25}(7)$ \\
\hline 47 & 1013.7 & 85.1 & 2.0 & $v_{\mathrm{C} 8 \mathrm{C} 17}(12), v_{\mathrm{C}_{2} \mathrm{C} 20}(8), v_{\mathrm{C}_{2} \mathrm{~N} 3}(7), v_{\mathrm{C}_{11} \mathrm{C} 12}(6), \rho_{\mathrm{C} 2 \mathrm{H} 22 \mathrm{H} 21}(6), v_{\mathrm{C}_{13} \mathrm{C} 15}(5)$ \\
\hline 48 & 1016.1 & 0.0 & 0.0 & $\omega_{\mathrm{C} 15 \mathrm{H} 29}(30), \omega_{\mathrm{C} 13 \mathrm{H} 27}(28), \omega_{\mathrm{C} 14 \mathrm{H} 28}(15), \omega_{\mathrm{C} 12 \mathrm{H} 26}(14),{ }^{3} \tau_{1 / R 6}(12)$ \\
\hline 49 & 1052.6 & 3.9 & 0.2 & $\begin{array}{l}\rho_{\mathrm{C} 2 \mathrm{H} 22 \mathrm{H} 21}(28), \tau_{\mathrm{C} 20 \mathrm{H} 31 \mathrm{H} 32}(15), v_{\mathrm{C} 2 \mathrm{C} 20}(13), \beta_{\mathrm{C} 20 \mathrm{~S} 1 \mathrm{H} 33}(10), \\
\rho_{\mathrm{C} 20 \mathrm{H} 31 \mathrm{H} 32}(7)\end{array}$ \\
\hline 50 & 1057.8 & 0.8 & 4 & $v_{\mathrm{C}_{13} \mathrm{C} 15}(32), v_{\mathrm{C} 14 \mathrm{C} 15}(12), \rho_{\mathrm{C} 12 \mathrm{H} 26}(11), \rho_{\mathrm{C} 14 \mathrm{H} 28}(11), v_{\mathrm{C} 12 \mathrm{C} 13}(10)$ \\
\hline 51 & 1100.6 & 23.9 & 1.0 & $v_{\mathrm{C} 6 \mathrm{C} 19}(29), \rho_{\mathrm{C} 19 \mathrm{H} 30}(16),{ }^{1} \beta_{\text {trig/R6 }}(12), v_{\mathrm{C} 6 \mathrm{C} 7}(7)$ \\
\hline 52 & 1113.2 & 1.0 & 0.4 & $\begin{array}{l}{ }^{3} \beta_{\text {trig/R6 }}(37), v_{\mathrm{C} 12 \mathrm{C} 13}(9), v_{\mathrm{C} 14 \mathrm{C} 15}(8), \rho_{\mathrm{C} 13 \mathrm{H} 27}(8), \rho_{\mathrm{C} 15 \mathrm{H} 29}(7), \\
v_{\mathrm{C} 9 \mathrm{C} 10}(6), \rho_{\mathrm{C} 14 \mathrm{H} 28}(6)\end{array}$ \\
\hline 53 & 1125.8 & 2.1 & 2.4 & $v_{\mathrm{C} 2 \mathrm{~N} 3}(34), \tau_{\mathrm{C} 20 \mathrm{H} 31 \mathrm{H} 32}(11), v_{\mathrm{C} 2 \mathrm{C} 20}(11), \rho_{\mathrm{C} 18 \mathrm{~N} 3}(7), v_{\mathrm{C} 8 \mathrm{C} 17}(6)$ \\
\hline 54 & 1176.3 & 4.9 & 3.5 & $\begin{array}{l}\rho_{\mathrm{C} 13 \mathrm{H} 27}(18), \rho_{\mathrm{C} 12 \mathrm{H} 26}(12), v_{\mathrm{C} 9 \mathrm{C} 16}(8),{ }^{1} \beta_{\text {trig/R6 }}(7), v_{\mathrm{C} 9 \mathrm{C} 10}(7), \\
{ }^{3} \beta_{\text {trig/R6 }}(6)\end{array}$ \\
\hline 55 & 1182.8 & 21.7 & 0.3 & $\begin{array}{l}\rho_{\mathrm{C} 15 \mathrm{H} 29}(28), \rho_{\mathrm{C} 14 \mathrm{H} 28}(10), v_{\mathrm{C} 14 \mathrm{C} 15}(8), \rho_{\mathrm{C} 13 \mathrm{H} 27}(7),{ }^{1} \beta_{\text {trig/R6 }}(6), \\
{ }^{3} \beta_{\text {trig/R6 }}(5), v_{\mathrm{C} 13 \mathrm{C} 15}(5)\end{array}$ \\
\hline 56 & 1198.1 & 13.8 & 6 & $\rho_{\mathrm{C} 6 \mathrm{H} 24}(19), \tau_{\mathrm{C} 20 \mathrm{H} 31 \mathrm{H} 32}(8), \rho_{\mathrm{C} 13 \mathrm{H} 27}(7), \rho_{\mathrm{C} 7 \mathrm{H} 25}(7), v_{\mathrm{C} 16 \mathrm{C} 18}(5)$ \\
\hline 57 & 1214.2 & 28.6 & 1.1 & $\tau_{\mathrm{C} 20 \mathrm{H} 31 \mathrm{H} 32}(23), \rho_{\mathrm{C} 7 \mathrm{H} 25}(18), \rho_{\mathrm{C} 6 \mathrm{H} 24}(17), \rho_{\mathrm{C} 19 \mathrm{H} 30}(11), v_{\mathrm{C} 6 \mathrm{C} 7}(7)$ \\
\hline 58 & 1219.1 & 28.5 & 2.2 & $\begin{array}{l}\tau_{\mathrm{C} 20 \mathrm{H} 31 \mathrm{H} 32}(17), \rho_{\mathrm{C} 19 \mathrm{H} 30}(15), v_{\mathrm{C} 2 \mathrm{~N} 3}(8), \rho_{\mathrm{C} 14 \mathrm{H} 28}(7), v_{\mathrm{C} 11 \mathrm{C} 17}(7), \\
{ }^{3} \beta_{\text {trig/R6 }}(5)\end{array}$ \\
\hline 59 & 1257.9 & 96.8 & 5.8 & $\begin{array}{l}\omega_{\mathrm{C} 20 \mathrm{H} 31 \mathrm{H} 32}(12), \tau_{\mathrm{C} 2 \mathrm{H} 22 \mathrm{H} 21}(10), v_{\mathrm{C} 7 \mathrm{C} 18}(10), \rho_{\mathrm{C} 12_{2} 26}(8), \tau_{\mathrm{C} 20 \mathrm{H} 31 \mathrm{H} 32}(7), \\
\rho_{\mathrm{C} 7 \mathrm{H} 25}(6)\end{array}$ \\
\hline 60 & 1271.6 & 41.6 & 0.1 & $\omega_{\mathrm{C} 20 \mathrm{H} 31 \mathrm{H} 32}(22), \tau_{\mathrm{C}_{2} \mathrm{H} 22 \mathrm{H} 21}(17), \rho_{\mathrm{C} 12 \mathrm{H} 26}(10), \rho_{\mathrm{C} 14 \mathrm{H} 28}(9),{ }^{2} \beta_{\text {trig/R6}}(8)$ \\
\hline 61 & 1291.1 & 564.4 & 9.4 & $\begin{array}{l}v_{\mathrm{C} 9 \mathrm{C} 10}(12), \omega_{\mathrm{C} 20 \mathrm{H} 31 \mathrm{H} 32}(9), v_{\mathrm{C} 9 \mathrm{C} 16}(8), v_{\mathrm{C} 8 \mathrm{C} 16}(7), \rho_{\mathrm{C} 14 \mathrm{H} 28}(6), \\
v_{\mathrm{C} 7 \mathrm{C} 18}(5), v_{\mathrm{C} 11 \mathrm{C} 17}(5), v_{\mathrm{C} 8 \mathrm{C} 17}(5), \rho_{\mathrm{C} 19 \mathrm{H} 30}(5)\end{array}$ \\
\hline 62 & 1318.2 & 116.5 & & $v_{\mathrm{C}_{11} \mathrm{C} 17}(19), v_{\mathrm{C} 8 \mathrm{C} 17}(14), \rho_{\mathrm{C} 12 \mathrm{H} 26}(8), \rho_{\mathrm{C} 17 \mathrm{O} 4}(8),{ }^{2} \beta_{2 / R 6}(7), \rho_{\mathrm{C} 13 \mathrm{H} 27}(5)$ \\
\hline 63 & 1325.9 & 149.6 & 6.8 & $\begin{array}{l}v_{\mathrm{N} 3 \mathrm{C} 18}(19), \omega_{\mathrm{C} 20 \mathrm{H} 31 \mathrm{H} 32}(19), \tau_{\mathrm{C} 2 \mathrm{H} 22 \mathrm{H} 21}(13), v_{\mathrm{C} 16 \mathrm{C} 18}(9), \rho_{\mathrm{N} 3 \mathrm{H} 23}(6), \\
{ }^{1} \beta_{\text {trig/R6}}(6)\end{array}$ \\
\hline 64 & 1350.1 & 79.5 & 0.8 & $\begin{array}{l}\tau_{\mathrm{C}_{2} \mathrm{H} 22 \mathrm{H} 21}(17), \omega_{\mathrm{C} 20 \mathrm{H} 31 \mathrm{H} 32}(15), v_{\mathrm{C} 9 \mathrm{C} 16}(9), v_{\mathrm{C} 16 \mathrm{C} 18}(9), \rho_{\mathrm{N} 3 \mathrm{H} 23}(8), \\
v_{\mathrm{N} 3 \mathrm{C} 18}(5)\end{array}$ \\
\hline 65 & 1358.7 & 25.7 & 1.0 & $\begin{array}{l}v_{\mathrm{C} 10 \mathrm{C} 14}(14), v_{\mathrm{C} 11 \mathrm{C} 12}(14), v_{\mathrm{C} 10 \mathrm{C} 11}(14), v_{\mathrm{C} 13 \mathrm{C} 15}(13), v_{\mathrm{C} 12 \mathrm{C} 13}(13), \\
v_{\mathrm{C} 14 \mathrm{C} 15}(12)\end{array}$ \\
\hline 66 & 1381.0 & 12.6 & 1.7 & $\begin{array}{l}v_{\mathrm{C} 16 \mathrm{C} 18}(15), v_{\mathrm{C} 8 \mathrm{C} 16}(13), v_{\mathrm{C} 8 \mathrm{C} 19}(12), v_{\mathrm{C} 6 \mathrm{C} 19}(7), v_{\mathrm{C} 9 \mathrm{C} 16}(6), v_{\mathrm{C} 6 \mathrm{C} 7}(6), \\
v_{\mathrm{C} 7 \mathrm{C} 18}(5), \rho_{\mathrm{C} 9 \mathrm{O} 5}(5)\end{array}$ \\
\hline 67 & 1393.5 & 17.7 & 1.6 & $\omega_{\mathrm{C} 2 \mathrm{H} 22 \mathrm{H} 21}(72), \delta_{1 / \mathrm{C}_{2} \mathrm{H} 22 \mathrm{H} 21}(6)$ \\
\hline 68 & 1438.2 & 39.1 & 0.5 & $\rho_{\mathrm{C} 7 \mathrm{H} 25}(26), v_{\mathrm{C} 8 \mathrm{C} 19}(11), v_{\mathrm{C} 6 \mathrm{C} 7}(11), v_{\mathrm{C} 8 \mathrm{C} 16}(7), v_{\mathrm{C} 9 \mathrm{C} 16}(6)$ \\
\hline 69 & 1477.2 & 11.4 & 1.8 & $\delta_{1 / \mathrm{C}_{20} \mathrm{H} 31 \mathrm{H} 32}(82)$ \\
\hline 70 & 1478.6 & 2.0 & 3.0 & $\rho_{\mathrm{C} 15 \mathrm{H} 29}(14), \rho_{\mathrm{C} 13 \mathrm{H} 27}(12), \delta_{1 / \mathrm{C} 20 \mathrm{H} 31 \mathrm{H} 32}(11), v_{\mathrm{C} 10 \mathrm{C} 14}(7), \rho_{\mathrm{C} 19 \mathrm{H} 30}(6)$, \\
\hline
\end{tabular}




\begin{tabular}{|c|c|c|c|c|}
\hline & & & & $v_{\mathrm{C} 11 \mathrm{C} 12}(6)$ \\
\hline 71 & 1488.5 & 8.6 & 0.9 & $\begin{array}{l}\rho_{\mathrm{C} 19 \mathrm{H} 30}(11), \delta_{1 / \mathrm{C}_{2} \mathrm{H} 22 \mathrm{H} 21}(8), v_{\mathrm{C} 8 \mathrm{C} 16}(8), \rho_{\mathrm{C} 13 \mathrm{H} 27}(7), \rho_{\mathrm{C} 15 \mathrm{H} 29}(6), \\
\rho_{\mathrm{C} 6 \mathrm{H} 24}(6), v_{\mathrm{C} 8 \mathrm{C} 19}(5), v_{\mathrm{C} 8 \mathrm{C} 17}(5)\end{array}$ \\
\hline 72 & 1502.9 & 5.7 & 1.4 & $\delta_{1 / \mathrm{C}_{2} \mathrm{H} 22 \mathrm{H} 21}(76)$ \\
\hline 73 & 1508.1 & 2.3 & 1.7 & $\rho_{\mathrm{C} 14 \mathrm{H} 28}(19), \rho_{\mathrm{C} 12 \mathrm{H} 26}(19), v_{\mathrm{C} 10 \mathrm{C} 11}(16), v_{\mathrm{C} 13 \mathrm{C} 15}(8)$ \\
\hline 74 & 1559.1 & 161.2 & 1.3 & $v_{\mathrm{C} 6 \mathrm{C} 19}(21), \rho_{\mathrm{C} 6 \mathrm{H} 24}(14), v_{\mathrm{N}_{3} \mathrm{C} 18}(14), \rho_{\mathrm{N}_{3} \mathrm{H}_{23}}(13), v_{\mathrm{C} 6 \mathrm{C} 7}(9), v_{\mathrm{C} 8 \mathrm{C} 16}(5)$ \\
\hline 75 & 1610.7 & 25.9 & 10.1 & $\begin{array}{l}v_{\mathrm{C} 13 \mathrm{C} 15}(15), v_{\mathrm{C} 10 \mathrm{C} 11}(15), v_{\mathrm{O} 5 \mathrm{C} 9}(14), v_{\mathrm{C} 12 \mathrm{C} 13}(8),{ }^{3} \beta_{3 / R 6}(7), \\
v_{\mathrm{C} 10 \mathrm{C} 14}(6), \rho_{\mathrm{C} 13 \mathrm{H} 27}(6)\end{array}$ \\
\hline 76 & 1618.4 & 54.0 & 16.7 & $\rho_{\mathrm{N} 3 \mathrm{H} 23}(17), v_{\mathrm{C} 8 \mathrm{C} 19}(13), v_{\mathrm{C} 7 \mathrm{C} 18}(8), v_{\mathrm{C} 6 \mathrm{C} 19}(7), v_{\mathrm{C} 16 \mathrm{C} 18}(6)$ \\
\hline 77 & 1628.4 & 57.2 & 10.4 & $\begin{array}{l}v_{\mathrm{C} 14 \mathrm{C} 15}(18), v_{\mathrm{C} 11 \mathrm{C} 12}(14), v_{\mathrm{C} 12 \mathrm{C} 13}(10),{ }^{3} \beta_{2 / R 6}(9), v_{\mathrm{C} 10 \mathrm{C} 14}(7), \\
\rho_{\mathrm{N} 3 \mathrm{H} 23}(5), \rho_{\mathrm{C} 12 \mathrm{H} 26}(5)\end{array}$ \\
\hline 78 & 1633.9 & 314.2 & 3.7 & $v_{\mathrm{C} 6 \mathrm{C} 7}(20), \rho_{\mathrm{N} 3 \mathrm{H} 23}(15), v_{\mathrm{N} 3 \mathrm{C} 18}(9), \rho_{\mathrm{C} 7 \mathrm{H} 25}(7),{ }^{1} \beta_{3 / R 6}(6), v_{\mathrm{C} 8 \mathrm{C} 19}(6)$ \\
\hline 79 & 1667.1 & 196.4 & 23.7 & $v_{\mathrm{O} 5 \mathrm{C} 9}(40),{ }^{2} \beta_{3 / R 6}(6),{ }^{2} \beta_{\text {trig/R6 }}(6)$ \\
\hline 80 & 1719.7 & 177.6 & 26.9 & $v_{\mathrm{O} 4 \mathrm{C} 17}(70),{ }^{2} \beta_{\text {trig/R6 }}(9)$ \\
\hline 81 & 2673.4 & 8.5 & 16.7 & $v_{\mathrm{S} 1 \mathrm{H} 33}(100)$ \\
\hline 82 & 3024.1 & 36.6 & 9.3 & $v_{\mathrm{C} 2 \mathrm{H} 22}(90), v_{\mathrm{C}_{2} \mathrm{H} 21}(6)$ \\
\hline 83 & 3052.6 & 40.3 & 12.6 & $v_{\mathrm{C} 20 \mathrm{H} 32}(48), v_{\mathrm{C} 20 \mathrm{H} 31}(47)$ \\
\hline 84 & 3091.5 & 5.3 & 3.8 & $v_{\mathrm{C} 2 \mathrm{H} 21}(66), v_{\mathrm{C} 20 \mathrm{H} 31}(18), v_{\mathrm{C} 20 \mathrm{H} 32}(8), v_{\mathrm{C}_{2} \mathrm{H} 22}(7)$ \\
\hline 85 & 3108.8 & 19.5 & 5.0 & $v_{\mathrm{C}_{20} \mathrm{H} 32}(42), v_{\mathrm{C}_{20 \mathrm{H} 31}}(32), v_{\mathrm{C}_{2} \mathrm{H} 21}(23)$ \\
\hline 86 & 3169.5 & 3.6 & 4.4 & $v_{\mathrm{C}_{15 \mathrm{H}} 29}(50), v_{\mathrm{C} 13 \mathrm{H} 27}(44)$ \\
\hline 87 & 3173.5 & 11.3 & 7.5 & $v_{\mathrm{C} 6 \mathrm{H} 24}(91)$ \\
\hline 88 & 3184.5 & 14.5 & 14.6 & $v_{\mathrm{C}_{13 \mathrm{H}} 27}(44), v_{\mathrm{C} 15 \mathrm{H} 29}(42), v_{\mathrm{C}_{12 \mathrm{H} 26}}(8)$ \\
\hline 89 & 3202.1 & 3.8 & 6.5 & $v_{\mathrm{C} 12 \mathrm{H} 26}(82), v_{\mathrm{C} 14 \mathrm{H} 28}(9), v_{\mathrm{C} 13 \mathrm{H} 27}(8)$ \\
\hline 90 & 3206.3 & 9.3 & 11.1 & $v_{\mathrm{C} 14 \mathrm{H} 28}(84), v_{\mathrm{C} 15 \mathrm{H} 29}(7), v_{\mathrm{C} 12 \mathrm{H} 26}(6)$ \\
\hline 91 & 3210.1 & 4.8 & 5.8 & $v_{\mathrm{C} 7 \mathrm{H} 25}(75), v_{\mathrm{C} 19 \mathrm{H} 30}(22)$ \\
\hline 92 & 3213.5 & 6.6 & 15.3 & $v_{\mathrm{C} 19 \mathrm{H} 30}(74), v_{\mathrm{C} 7 \mathrm{H} 25}(19), v_{\mathrm{C} 6 \mathrm{H} 24}(6)$ \\
\hline 93 & 3430.6 & 114.6 & 33.4 & $v_{\mathrm{N} 3 \mathrm{H} 23}(99)$ \\
\hline
\end{tabular}


Table S.8. Frequencies of normal vibrations, IR and Raman intensities and PEDs for $A c-G G$ conformer calculated at the B3LYP/6-311++G(d,p) level of theory.

\begin{tabular}{|c|c|c|c|c|}
\hline $\mathbf{N r}$ & $\begin{array}{l}\text { Freq. } \\
{\left[\mathrm{cm}^{-1}\right]}\end{array}$ & IR Int. & $\begin{array}{c}\text { Raman } \\
\text { Int. }\end{array}$ & PED $($ contributions $>5 \%)$ \\
\hline 1 & 24.9 & 0.5 & 10.4 & $\tau_{\mathrm{N} 3 \mathrm{C} 18}(40), \omega_{\mathrm{N} 3 \mathrm{H} 23}(28),{ }^{2} \tau_{3 / R 6}(7),{ }^{2} \tau_{2 / R 6}(5)$ \\
\hline 2 & 36.8 & 0.5 & 5.7 & $\tau_{\mathrm{C} 2 \mathrm{~N} 3}(38), \tau_{\mathrm{N} 3 \mathrm{C} 18}(21), \omega_{\mathrm{N} 3 \mathrm{H} 23}(11),{ }^{1} \tau_{3 / R 6}(11),{ }^{2} \tau_{3 / R 6}(7)$ \\
\hline 3 & 46.7 & 5.2 & 1.1 & ${ }^{2} \tau_{3 / R \sigma}(39), \tau_{\mathrm{C} 2 \mathrm{~N} 3}(20),{ }^{2} \tau_{2 / R \sigma}(14),{ }^{1} \tau_{3 / R \sigma}(7)$ \\
\hline 4 & 71.7 & 0.8 & 0.4 & ${ }^{2} \tau_{2 / R 6}(24), \tau_{\mathrm{C}_{2} \mathrm{C} 20}(18),{ }^{1} \tau_{3 / R 6}(13), \omega_{\mathrm{N} 3 \mathrm{H} 23}(12)$ \\
\hline 5 & 110.3 & 0.8 & 0.5 & $\tau_{\mathrm{C}_{2} \mathrm{C} 20}(26),{ }^{2} \tau_{2 / R 6}(17), \omega_{\mathrm{N}_{3} \mathrm{H} 23}(14),{ }^{3} \tau_{2 / R 6}(7), \tau_{\mathrm{N}_{3} \mathrm{C} 18}(6), \beta_{\mathrm{C}_{18 \mathrm{~N} 3 \mathrm{C} 2}}(5)$ \\
\hline 6 & 122.7 & 0.8 & 1.1 & ${ }^{2} \tau_{1 / R 6}(37),{ }^{3} \tau_{2 / R 6}(11), \omega_{\mathrm{N}_{3} \mathrm{H}_{23}}(9), \tau_{\mathrm{C}_{2} \mathrm{C} 20}(7),{ }^{3} \tau_{3 / R 6}(6)$ \\
\hline 7 & 151.5 & 1.4 & 0.6 & $\delta_{2 / \mathrm{C} 20 \mathrm{H} 31 \mathrm{H} 32}(20), \omega_{\mathrm{N}_{3} \mathrm{H}_{23}}(17), \rho_{\mathrm{C} 18 \mathrm{~N} 3_{3}}(11), \delta_{2 / \mathrm{C}_{2} \mathrm{H} 22 \mathrm{H} 21}(7),{ }^{2} \tau_{1 / R 6}(7)$ \\
\hline 8 & 166.4 & 0.4 & 0.0 & ${ }^{4} b f l_{R R}(24),{ }^{2} b f l_{R R}(23), \tau_{N 3 C l 8}(9),{ }^{2} \tau_{3 / R 6}(8), \omega_{C l 7 O 4}(6), \omega_{C 9 O 5}(6)$ \\
\hline 9 & 196.4 & 0.1 & 0.9 & ${ }^{\mathrm{I}} \tau_{2 / R 6}(25),{ }^{\mathrm{l}} \tau_{3 / R 6}(25),{ }^{4} b f l_{R R}(13), \omega_{C 19 H 30}(6)$ \\
\hline 10 & 230.4 & 6.2 & 0.2 & $\delta_{2 / \mathrm{C}_{20} \mathrm{H}_{31 \mathrm{H}} 2}(19), \delta_{2 / \mathrm{C}_{2} \mathrm{H}_{22} \mathrm{H}_{21}}(15), \tau_{\mathrm{S} 1 \mathrm{C} 20_{2}}(9),{ }^{3} \beta_{2 / R 6}(6)$ \\
\hline 11 & 258.3 & 3.6 & 0.9 & ${ }^{2} b f l_{R R}(28), \omega_{C 18 N 3}(11),{ }^{4} b f l_{R R}(7), \omega_{N 3 H 23}(7)$ \\
\hline 12 & 275.5 & 13.2 & 0.2 & $\tau_{\mathrm{S} 1 \mathrm{C} 20}(60),{ }^{2} \beta_{2 / R 6}(8), \rho_{\mathrm{C} 18 \mathrm{~N} 3}(7)$ \\
\hline 13 & 309.0 & 10.4 & 0.6 & ${ }^{2} \beta_{2 / R 6}(26), \delta_{2 / \mathrm{C} 20 \mathrm{H} 31 \mathrm{H} 32}(14), \beta_{\mathrm{C} 18 \mathrm{~N} 3 \mathrm{C} 2_{2}}(12), \tau_{\mathrm{S} 1 \mathrm{C} 20}(10), v_{\mathrm{C} 16 \mathrm{C} 18}(6)$ \\
\hline 14 & 323.5 & 2.1 & 0.5 & 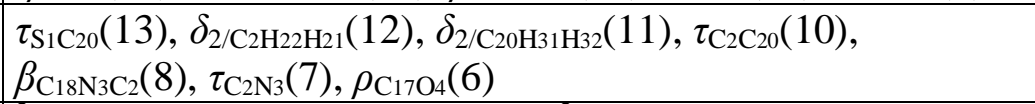 \\
\hline 15 & 350.3 & 1.5 & 0.0 & ${ }^{2} \beta_{3 / R 6}(25), \rho_{\mathrm{C} 18 \mathrm{~N} 3}(15), \rho_{\mathrm{C} 17 \mathrm{O} 4}(11),{ }^{2} \beta_{2 / R 6}(6), v_{\mathrm{C} 8 \mathrm{C} 17}(5)$ \\
\hline 16 & 369.7 & 10.2 & 1.2 & $\begin{array}{l}{ }^{2} \beta_{3 / R 6}(16),{ }^{1} \beta_{3 / R 6}(12), \rho_{\mathrm{C} 17 \mathrm{O} 4}(9), v_{\mathrm{C} 11 \mathrm{C} 17}(8), v_{\mathrm{C} 9 \mathrm{C} 16}(8), \rho_{\mathrm{C} 18 \mathrm{~N} 3}(7), \\
\delta_{2 / \mathrm{C} 20 \mathrm{H} 31 \mathrm{H} 32}(6)\end{array}$ \\
\hline 17 & 427.6 & 0.4 & 0.1 & ${ }^{3} \tau_{3 / R 6}(59),{ }^{4} b f l_{R R}(14)$ \\
\hline 18 & 429.7 & 21.5 & 0.6 & $\rho_{\mathrm{C} 9 \mathrm{O} 5}(37), \rho_{\mathrm{C} 17 \mathrm{O} 4}(15), v_{\mathrm{C} 9 \mathrm{C} 16}(8),{ }^{1} \beta_{3 / R 6}(8)$ \\
\hline 19 & 434.1 & 0.2 & 0.1 & ${ }^{3} \tau_{2 / R \sigma}(32),{ }^{1} \tau_{2 / R \sigma}(20),{ }^{1} \tau_{3 / R 6}(17), \omega_{\mathrm{C} 9 \mathrm{O} 5}(6),{ }^{2} \tau_{1 / R 6}(6)$ \\
\hline 20 & 445.6 & 0.6 & 0.3 & $\begin{array}{l}{ }^{1} \beta_{2 / R 6}(15), \delta_{2 / \mathrm{C}_{2} \mathrm{H}_{22 \mathrm{H} 21}}(14),{ }^{2} \beta_{2 / R 6}(13),{ }^{2} \beta_{3 / R 6}(10),{ }^{3} \beta_{2 / R 6}(7), \\
\delta_{2 / \mathrm{C} 20 \mathrm{H} 31 \mathrm{H} 32}(7)\end{array}$ \\
\hline 21 & 476.9 & 2.8 & 9.0 & $\begin{array}{l}{ }^{2} \beta_{2 / R 6}(28),{ }^{2} \beta_{3 / R \sigma}(21),{ }^{3} \beta_{3 / R 6}(9),{ }^{1} \beta_{3 / R 6}(8), v_{\mathrm{C} 8 \mathrm{C} 16}(6), v_{\mathrm{C} 10 \mathrm{C} 11}(5), \\
v_{\mathrm{C} 11 \mathrm{C} 17}(5)\end{array}$ \\
\hline 22 & 490.7 & 1.2 & 0.2 & ${ }^{3} \tau_{2 / R \sigma}(22),{ }^{1} \tau_{3 / R \sigma}(22),{ }^{1} \tau_{2 / R \sigma}(10),{ }^{2} \tau_{2 / R 6}(9),{ }^{3} \tau_{3 / R \sigma}(6),{ }^{1} \tau_{1 / R \sigma}(6)$ \\
\hline 23 & 504.5 & 2.3 & 1.8 & $\delta_{2 / \mathrm{C} 2 \mathrm{H} 22 \mathrm{H} 21}(23), \rho_{\mathrm{C} 18 \mathrm{~N} 3}(10), \rho_{\mathrm{C} 20 \mathrm{H} 31 \mathrm{H} 32}(8),{ }^{1} \beta_{2 / R 6}(8),{ }^{3} \beta_{2 / R 6}(5)$ \\
\hline 24 & 553.5 & 6.9 & 0.2 & $\omega_{\mathrm{C} 18 \mathrm{~N}_{3}}(31),{ }^{1} \tau_{3 / R \sigma}(14),{ }^{1} \tau_{2 / R \sigma}(13),{ }^{1} \tau_{1 / R 6}(8),{ }^{2} b f l_{R R}(7)$ \\
\hline 25 & 601.9 & 6.2 & 0.7 & $\begin{array}{l}{ }^{1} \beta_{3 / R 6}(17),{ }^{1} \beta_{2 / R 6}(12),{ }^{3} \beta_{2 / R 6}(11), \beta_{\mathrm{C} 18 \mathrm{~N} 3 \mathrm{C} 2_{2}}(11), \rho_{\mathrm{C} 18 \mathrm{~N} 3}(7), \\
{ }_{3}^{3} \beta_{3 / R 6}(7)\end{array}$ \\
\hline 26 & 630.9 & 1.0 & 0.1 & ${ }^{2} \beta_{\text {trig/R6 }}(43),{ }^{1} \beta_{2 / R 6}(15), \rho_{\mathrm{C} 18 \mathrm{~N} 3}(6), \beta_{\mathrm{C} 18 \mathrm{~N} 3 \mathrm{C} 2}(5)$ \\
\hline 27 & 650.9 & 7.8 & 2.0 & $v_{\mathrm{S}_{1} \mathrm{C}_{20}}(65), \delta_{2 / \mathrm{C}_{20} \mathrm{H}_{31} \mathrm{H}_{32}(7)}$ \\
\hline 28 & 670.6 & 1.9 & 0.2 & ${ }^{3} \tau_{1 / R 6}(37),{ }^{1} \tau_{1 / R 6}(19), \omega_{\mathrm{C} 18 \mathrm{~N} 3}(12), \omega_{\mathrm{C} 9 \mathrm{OO}}(11)$ \\
\hline 29 & 677.0 & 12.6 & 1.1 & ${ }^{3} \beta_{3 / R \sigma}(36),{ }^{3} \beta_{2 / R 6}(9), v_{\mathrm{C} 10 \mathrm{C} 11}(7)$ \\
\hline 30 & 705.0 & 1.5 & 0.5 & $\rho_{\mathrm{C} 9 \mathrm{O} 5}(21),{ }^{3} \beta_{2 / R 6}(18), \rho_{\mathrm{C} 17 \mathrm{O} 4}(18),{ }^{1} \beta_{2 / R 6}(11),{ }^{1} \beta_{3 / R 6}(8),{ }^{3} \beta_{3 / R 6}(5)$ \\
\hline 31 & 723.3 & 51.2 & 0.1 & ${ }^{1} \tau_{1 / R 6}(35),{ }^{3} \tau_{1 / R 6}(25), \omega_{\mathrm{C} 6 \mathrm{H} 24}(11), \omega_{\mathrm{C} 18 \mathrm{~N} 3}(6)$ \\
\hline 32 & 732.3 & 9.3 & 0.2 & $\begin{array}{l}\omega_{\mathrm{C} 9 \mathrm{O} 5}(13), \omega_{\mathrm{C}_{17} \mathrm{O} 4}(11), \omega_{\mathrm{C} 15 \mathrm{H} 29}(10), \omega_{\mathrm{N} 3 \mathrm{H} 23}(9), \tau_{\mathrm{N} 3 \mathrm{C} 18}(9), \\
\omega_{\mathrm{C}_{33 \mathrm{H}} 2}(7), \omega_{\mathrm{C}_{12} \mathrm{H} 26}(6), \tau_{\mathrm{C}_{2} \mathrm{~N} 3}(5)\end{array}$ \\
\hline 33 & 753.0 & 70.4 & 0.2 & $\begin{array}{l}\rho_{\mathrm{C} 20 \mathrm{H} 31 \mathrm{H} 32}(13), \beta_{\mathrm{C}_{20} \mathrm{~S}_{1 \mathrm{H} 33}}(10), \omega_{\mathrm{C} 18 \mathrm{~N} 3}(9), \omega_{\mathrm{C} 13 \mathrm{H} 27}(9),{ }^{1} \tau_{1 / R 6}(8), \\
{ }^{3} \tau_{1 / R 6}(6)\end{array}$ \\
\hline 34 & 771.7 & 53.2 & 0.4 & $\begin{array}{l}\rho_{\mathrm{C}_{20 \mathrm{H} 31 \mathrm{H} 32}}(20), \beta_{\mathrm{C} 20 \mathrm{~S} 1 \mathrm{H} 33}(18), \omega_{\mathrm{N}_{3} \mathrm{H} 23}(13),{ }^{3} \tau_{1 / R 6}(7), \omega_{\mathrm{C} 18 \mathrm{~N} 3}(6), \\
\tau_{\mathrm{N} 3 \mathrm{C} 18}(6)\end{array}$ \\
\hline 35 & 791.1 & 0.5 & 0.1 & $\begin{array}{l}{ }^{3} \tau_{1 / R 6}(14), \omega_{\mathrm{C} 7 \mathrm{H} 25}(13), \omega_{\mathrm{C} 19 \mathrm{H} 30}(11),{ }^{2} \tau_{1 / R 6}(11), \omega_{\mathrm{C} 6 \mathrm{H} 24}(11), \\
\omega_{\mathrm{C} 17 \mathrm{O} 4}(10), \omega_{\mathrm{C} 15 \mathrm{H} 29}(7), \omega_{\mathrm{C} 95}(7)\end{array}$ \\
\hline 36 & 792.2 & 2.3 & 1.0 & ${ }^{1} \beta_{\text {trig/R6 }}(18),{ }^{1} \beta_{3 / R 6}(16),{ }^{3} \beta_{2 / R 6}(13),{ }^{3} \beta_{\text {trig/R6 }}(8), v_{\mathrm{C} 9 \mathrm{C} 10}(6)$, \\
\hline
\end{tabular}




\begin{tabular}{|c|c|c|c|c|}
\hline & & & & ${ }^{2} \beta_{\text {trig/R6 }}(5)$ \\
\hline 37 & 810.9 & 0.7 & 0.1 & $\begin{array}{l}\omega_{\mathrm{C} 9 \mathrm{O} 5}(29), \omega_{\mathrm{C} 13 \mathrm{H} 27}(13),{ }^{1} \tau_{1 / R 6}(11),{ }^{2} \tau_{1 / R 6}(9), \omega_{\mathrm{C} 18 \mathrm{~N} 3}(8), \\
\omega_{\mathrm{C} 15 \mathrm{H} 29}(6)\end{array}$ \\
\hline 38 & 829.2 & 23.7 & 0.0 & $\begin{array}{l}\omega_{\mathrm{C} 7 \mathrm{H} 25}(22), \omega_{\mathrm{C} 17 \mathrm{O} 4}(18),{ }^{1} \tau_{1 / R 6}(15), \omega_{\mathrm{C} 18 \mathrm{~N} 3}(8),{ }^{2} \tau_{1 / R 6}(7), \\
\omega_{\mathrm{C} 6 \mathrm{H} 24}(7), \omega_{\mathrm{C} 15 \mathrm{H} 29}(6)\end{array}$ \\
\hline 39 & 861.4 & 14.6 & 1.7 & $\begin{array}{l}{ }^{1} \beta_{2 / R 6}(9), v_{\mathrm{C} 2 \mathrm{~N} 3}(8),{ }^{2} \beta_{\text {trig/R6}}(6), v_{\mathrm{C} 16 \mathrm{C} 18}(6), v_{\mathrm{N} 3 \mathrm{C} 18}(6), \rho_{\mathrm{C} 17 \mathrm{O} 4}(6), \\
v_{\mathrm{C} 7 \mathrm{C} 18}(5)\end{array}$ \\
\hline 40 & 914.5 & 0.2 & 0.0 & $\begin{array}{l}\omega_{\mathrm{C} 14 \mathrm{H} 28}(24), \omega_{\mathrm{C} 12 \mathrm{H} 26}(24), \omega_{\mathrm{C} 19 \mathrm{H} 30}(12), \omega_{\mathrm{C} 15 \mathrm{H} 29}(11), \omega_{\mathrm{C} 13 \mathrm{H} 27}(10), \\
\omega_{\mathrm{C} 7 \mathrm{H} 25}(9)\end{array}$ \\
\hline 41 & 922.4 & 0.8 & 0.0 & $\omega_{\mathrm{C} 19 \mathrm{H} 30}(38), \omega_{\mathrm{C} 7 \mathrm{H} 25}(25), \omega_{\mathrm{C} 14 \mathrm{H} 28}(7), \omega_{\mathrm{C} 12 \mathrm{H} 26}(6)$ \\
\hline 42 & 928.4 & 8.9 & 1.4 & ${ }^{1} \beta_{\text {trig/R6}}(18),{ }^{3} \beta_{\text {trig/R6 }}(18), \rho_{\mathrm{C} 9 \mathrm{O} 5}(17), v_{\mathrm{C} 16 \mathrm{C} 18}(10), v_{\mathrm{C} 9 \mathrm{C} 10}(5)$ \\
\hline 43 & 933.7 & 12.9 & 0.3 & $\begin{array}{l}v_{\mathrm{C} 2 \mathrm{C} 20}(26), \rho_{\mathrm{C} 17 \mathrm{O} 4}(10),{ }^{1} \beta_{\text {trig/R6 }}(8),{ }^{3} \beta_{\text {trig/R6 }}(7), \rho_{\mathrm{C} 2 \mathrm{H} 22 \mathrm{H} 21}(6), \\
\beta_{\mathrm{C}_{20 \mathrm{~S} 1 \mathrm{H} 33}(5)}\end{array}$ \\
\hline 44 & 974.9 & 3.2 & 0.5 & $\rho_{\mathrm{C} 2 \mathrm{H} 22 \mathrm{H} 21}(37), \beta_{\mathrm{C}_{20} \mathrm{~S}_{1 \mathrm{H}} 33}(24), \tau_{\mathrm{C} 20 \mathrm{H} 31 \mathrm{H} 32}(8), \delta_{2 / \mathrm{C} 20 \mathrm{H} 31 \mathrm{H} 32}(5)$ \\
\hline 45 & 1002.4 & 0.3 & 0.0 & $\begin{array}{l}\omega_{\mathrm{C} 6 \mathrm{H} 24}(33), \omega_{\mathrm{C} 12 \mathrm{H} 26}(14), \omega_{\mathrm{C} 19 \mathrm{H} 30}(13), \omega_{\mathrm{C} 14 \mathrm{H} 28}(12), \omega_{\mathrm{C} 7 \mathrm{H} 25}(8), \\
\omega_{\mathrm{C} 13 \mathrm{H} 27}(5)\end{array}$ \\
\hline 46 & 1004.1 & 2.4 & 0.0 & $\begin{array}{l}\omega_{\mathrm{C} 6 \mathrm{H} 24}(23), \omega_{\mathrm{C} 12 \mathrm{H} 26}(20), \omega_{\mathrm{C} 14 \mathrm{H} 28}(18), \omega_{\mathrm{C} 19 \mathrm{H} 30}(10), \omega_{\mathrm{C} 13 \mathrm{H} 27}(7), \\
\omega_{\mathrm{C} 15 \mathrm{H} 29}(6), \omega_{\mathrm{C} 7 \mathrm{H} 25}(5)\end{array}$ \\
\hline 47 & 1014.2 & 69.0 & 1.1 & $v_{\mathrm{C}_{2} \mathrm{C} 20}(17), v_{\mathrm{C} 8 \mathrm{C} 17}(13), v_{\mathrm{C}_{11 \mathrm{C}} 12}(7), v_{\mathrm{C}_{2 \mathrm{~N}} 3}(6), v_{\mathrm{C} 13 \mathrm{C} 15}(5), \rho_{\mathrm{C} 17 \mathrm{O} 4}(5)$ \\
\hline 48 & 1017.5 & 0.0 & 0.0 & $\omega_{\mathrm{C} 15 \mathrm{H} 29}(30), \omega_{\mathrm{C} 13 \mathrm{H} 27}(28), \omega_{\mathrm{C} 14 \mathrm{H} 28}(16), \omega_{\mathrm{C} 12 \mathrm{H} 26}(13),{ }^{3} \tau_{1 / R 6}(12)$ \\
\hline 49 & 1052.8 & 21.0 & 5.9 & $v_{\mathrm{C}_{2} \mathrm{C} 20}(20), \rho_{\mathrm{C} 20 \mathrm{H} 31 \mathrm{H} 32}(15), \rho_{\mathrm{C} 2 \mathrm{H} 22 \mathrm{H} 21}(15), \beta_{\mathrm{C}_{20} \mathrm{~S}_{1} \mathrm{H} 33}(8), v_{\mathrm{C}_{13} \mathrm{C} 15}(8)$ \\
\hline 50 & 1059.3 & 3.2 & 1.8 & $\begin{array}{l}v_{\mathrm{C} 13 \mathrm{C} 15}(24), \rho_{\mathrm{C} 12 \mathrm{H} 26}(9), \rho_{\mathrm{C} 14 \mathrm{H} 28}(9), v_{\mathrm{C} 14 \mathrm{C} 15}(9), v_{\mathrm{C} 12 \mathrm{C} 13}(7), \\
\rho_{\mathrm{C} 20 \mathrm{H} 31 \mathrm{H} 32}(7), \rho_{\mathrm{C} 2 \mathrm{H} 22 \mathrm{H} 21}(6)\end{array}$ \\
\hline 51 & 1098.0 & 21.4 & 0.8 & $v_{\mathrm{C} 6 \mathrm{C} 19}(29), \rho_{\mathrm{C} 19 \mathrm{H} 30}(16),{ }^{1} \beta_{\text {trig/R6}}(14), v_{\mathrm{C} 6 \mathrm{C} 7}(7), v_{\mathrm{C} 2 \mathrm{~N} 3}(6)$ \\
\hline 52 & 1113.4 & 1.1 & 0.3 & $\begin{array}{l}{ }^{3} \beta_{\text {trig/R6 }}(37), v_{\mathrm{C} 12 \mathrm{C} 13}(9), v_{\mathrm{C} 14 \mathrm{C} 15}(8), \rho_{\mathrm{C} 13 \mathrm{H} 27}(8), \rho_{\mathrm{C} 15 \mathrm{H} 29}(7), \\
v_{\mathrm{C} 9 \mathrm{C} 10}(6), \rho_{\mathrm{C} 14 \mathrm{H} 28}(5), v_{\mathrm{C} 11 \mathrm{C} 17}(5)\end{array}$ \\
\hline 53 & 1130.8 & 0.9 & 2.9 & $v_{\mathrm{C}_{2} \mathrm{~N} 3}(39), \rho_{\mathrm{C} 18 \mathrm{~N} 3}(7), v_{\mathrm{C} 8 \mathrm{C} 17}(6), \tau_{\mathrm{C}_{20 \mathrm{H} 31 \mathrm{H} 32}}(6)$ \\
\hline 54 & 1176.4 & 5.3 & 3.6 & $\begin{array}{l}\rho_{\mathrm{C} 13 \mathrm{H} 27}(17), \rho_{\mathrm{C} 12 \mathrm{H} 26}(12), v_{\mathrm{C} 9 \mathrm{C} 16}(9),{ }^{1} \beta_{\text {trig/R6 }}(7), v_{\mathrm{C} 9 \mathrm{C} 10}(7), \\
{ }^{3} \beta_{\text {trig/R6}}(6)\end{array}$ \\
\hline 55 & 1183.6 & 19.9 & 0.3 & $\begin{array}{l}\rho_{\mathrm{C} 15 \mathrm{H} 29}(30), \rho_{\mathrm{C} 14 \mathrm{H} 28}(10), v_{\mathrm{C} 14 \mathrm{C} 15}(9), \rho_{\mathrm{C} 13 \mathrm{H} 27}(8), v_{\mathrm{C} 13 \mathrm{C} 15}(5), \\
{ }^{1} \beta_{\text {trig/R6 }}(5),{ }^{3} \beta_{\text {trig/R6 }}(5)\end{array}$ \\
\hline 56 & 1199.2 & 20.7 & 9.4 & $\rho_{\mathrm{C} 6 \mathrm{H} 24}(26), \rho_{\mathrm{C} 7 \mathrm{H} 25}(10), v_{\mathrm{C} 6 \mathrm{C} 7}(7), \rho_{\mathrm{C} 13 \mathrm{H} 27}(6)$ \\
\hline 57 & 1216.5 & 1.5 & 3.6 & $\rho_{\mathrm{C} 19 \mathrm{H} 30}(26), \rho_{\mathrm{C} 7 \mathrm{H} 25}(12), v_{\mathrm{C} 8 \mathrm{C} 19}(8), \rho_{\mathrm{C} 6 \mathrm{H} 24}(8), v_{\mathrm{C} 11 \mathrm{C} 17}(7), \rho_{\mathrm{C} 14 \mathrm{H} 28}(6)$ \\
\hline 58 & 1229.3 & 88.6 & 1.5 & $\tau_{\mathrm{C} 20 \mathrm{H} 31 \mathrm{H} 32}(32), \tau_{\mathrm{C} 2 \mathrm{H} 22 \mathrm{H} 21}(14), v_{\mathrm{C} 2 \mathrm{~N} 3}(7), v_{\mathrm{C} 7 \mathrm{C} 18}(6), \rho_{\mathrm{C} 7 \mathrm{H} 25}(5)$ \\
\hline 59 & 1262.9 & 79.8 & 3.7 & $\tau_{\mathrm{C} 20 \mathrm{H} 31 \mathrm{H} 32}(24), \omega_{\mathrm{C} 20 \mathrm{H} 31 \mathrm{H} 32}(8), \rho_{\mathrm{C} 12 \mathrm{H} 26}(7), v_{\mathrm{C} 7 \mathrm{C} 18}(6), \tau_{\mathrm{C} 2 \mathrm{H} 22 \mathrm{H} 21}(6)$ \\
\hline 60 & 1269.6 & 42.3 & 0.7 & $\begin{array}{l}\omega_{\mathrm{C} 20 \mathrm{H} 31 \mathrm{H} 32}(18), \tau_{\mathrm{C} 2 \mathrm{H} 22 \mathrm{H} 21}(17), \rho_{\mathrm{C} 12 \mathrm{H} 26}(10), \rho_{\mathrm{C} 14 \mathrm{H} 28}(9),{ }^{2} \beta_{\text {trig/R6 }}(8), \\
{ }^{1} \beta_{\text {trig/R6 }}(5)\end{array}$ \\
\hline 61 & 1291.0 & 540.5 & 10.1 & $\begin{array}{l}v_{\mathrm{C} 9 \mathrm{C} 10}(13), v_{\mathrm{C} 9 \mathrm{C} 16}(8), v_{\mathrm{C} 8 \mathrm{C} 16}(7), \omega_{\mathrm{C}_{20} \mathrm{H} 31 \mathrm{H} 32}(7), \rho_{\mathrm{C} 14 \mathrm{H} 28}(6), \\
v_{\mathrm{C} 7 \mathrm{C} 18}(6), v_{\mathrm{C} 16 \mathrm{C} 18}(5), \rho_{\mathrm{C} 19 \mathrm{H} 30}(5), v_{\mathrm{C} 11 \mathrm{C} 17}(5), v_{\mathrm{C} 8 \mathrm{C} 17}(5)\end{array}$ \\
\hline 62 & 1318.4 & 140.1 & 1.3 & $v_{\mathrm{C}_{11} \mathrm{C}_{17}}(19), v_{\mathrm{C} 8 \mathrm{C} 17}(15), \rho_{\mathrm{C} 12 \mathrm{H} 26}(9), \rho_{\mathrm{C} 17 \mathrm{O} 4}(7),{ }^{2} \beta_{2 / R 6}(7), \rho_{\mathrm{C} 13 \mathrm{H} 27}(5)$ \\
\hline 63 & 1324.9 & 138.4 & 5.8 & $\omega_{\mathrm{C} 20 \mathrm{H} 31 \mathrm{H} 32}(27), v_{\mathrm{N} 3 \mathrm{C} 18}(18), \tau_{\mathrm{C} 2 \mathrm{H} 22 \mathrm{H} 21}(9), v_{\mathrm{C} 16 \mathrm{C} 18}(8), \rho_{\mathrm{N} 3 \mathrm{H} 23}(6)$ \\
\hline 64 & 1350.0 & 74.1 & 0.8 & $\begin{array}{l}\tau_{\mathrm{C}_{2} \mathrm{H} 22 \mathrm{H} 21}(18), \omega_{\mathrm{C} 20 \mathrm{H} 31 \mathrm{H} 32}(16), v_{\mathrm{C} 9 \mathrm{C} 16}(9), v_{\mathrm{C} 16 \mathrm{C} 18}(9), \rho_{\mathrm{N} 3 \mathrm{H} 23}(8), \\
v_{\mathrm{N} 3 \mathrm{C} 18}(5)\end{array}$ \\
\hline 65 & 1358.9 & 23.8 & 1.1 & $\begin{array}{l}v_{\mathrm{C} 10 \mathrm{C} 14}(14), v_{\mathrm{C} 11 \mathrm{C} 12}(14), v_{\mathrm{C} 10 \mathrm{C} 11}(14), v_{\mathrm{C} 13 \mathrm{C} 15}(13), v_{\mathrm{C} 12 \mathrm{C} 13}(13), \\
v_{\mathrm{C} 14 \mathrm{C} 15}(12)\end{array}$ \\
\hline 66 & 1379.1 & 14.3 & 1.6 & $\begin{array}{l}v_{\mathrm{C} 16 \mathrm{C} 18}(14), v_{\mathrm{C} 8 \mathrm{C} 16}(13), v_{\mathrm{C} 8 \mathrm{C} 19}(11), v_{\mathrm{C} 6 \mathrm{C} 19}(7), v_{\mathrm{C} 9 \mathrm{C} 16}(6), v_{\mathrm{C} 7 \mathrm{C} 18}(5), \\
v_{\mathrm{C} 6 \mathrm{C} 7}(5)\end{array}$ \\
\hline 67 & 1390.9 & 14.2 & 2.0 & $\omega_{\mathrm{C} 2 \mathrm{H} 22 \mathrm{H} 21}(68), \delta_{1 / \mathrm{C} 2 \mathrm{H} 22 \mathrm{H} 21}(6)$ \\
\hline 68 & 1436.2 & 39.5 & 0.4 & $\rho_{\mathrm{C} 7 \mathrm{H} 25}(25), v_{\mathrm{C} 6 \mathrm{C} 7}(11), v_{\mathrm{C} 8 \mathrm{C} 19}(11), v_{\mathrm{C} 8 \mathrm{C} 16}(8), v_{\mathrm{C} 9 \mathrm{C} 16}(6)$ \\
\hline
\end{tabular}




\begin{tabular}{|c|c|c|c|c|}
\hline 69 & 1465.4 & 12.0 & 0.9 & $\delta_{1 / \mathrm{C}_{20 \mathrm{H} 31 \mathrm{H} 32}(91)}$ \\
\hline 70 & 1478.3 & 0.4 & 3.8 & $\begin{array}{l}\rho_{\mathrm{C} 15 \mathrm{H} 29}(15), \rho_{\mathrm{C} 13 \mathrm{H} 27}(13), \rho_{\mathrm{C} 19 \mathrm{H} 30}(7), v_{\mathrm{C} 10 \mathrm{C} 14}(7), v_{\mathrm{C} 11 \mathrm{C} 12}(6), \\
v_{\mathrm{C} 8 \mathrm{C} 16}(6), v_{\mathrm{O} 5 \mathrm{C} 9}(5)\end{array}$ \\
\hline 71 & 1488.2 & 9.4 & 0.8 & $\begin{array}{l}\delta_{1 / \mathrm{C} 2 \mathrm{H} 22 \mathrm{H} 21}(10), \rho_{\mathrm{C} 19 \mathrm{H} 30}(10), \rho_{\mathrm{C} 13 \mathrm{H} 27}(7), v_{\mathrm{C} 8 \mathrm{C} 16}(7), \rho_{\mathrm{C} 15 \mathrm{H} 29}(6), \\
\rho_{\mathrm{C} 6 \mathrm{H} 24}(6), v_{\mathrm{C} 8 \mathrm{C} 19}(5)\end{array}$ \\
\hline 72 & 1500.5 & 4.7 & 1.2 & $\delta_{1 / \mathrm{C}_{2} \mathrm{H} 22 \mathrm{H} 21}(74)$ \\
\hline 73 & 1508.1 & 2.5 & 1.9 & $\rho_{\mathrm{C} 14 \mathrm{H} 28}(20), \rho_{\mathrm{C} 12 \mathrm{H} 26}(19), v_{\mathrm{C} 10 \mathrm{C} 11}(17), v_{\mathrm{C} 13 \mathrm{C} 15}(8), \rho_{\mathrm{C} 13 \mathrm{H} 27}(5)$ \\
\hline 74 & 1556.9 & 163.5 & 1.7 & $v_{\mathrm{C} 6 \mathrm{C} 19}(21), \rho_{\mathrm{N} 3 \mathrm{H} 23}(14), \rho_{\mathrm{C} 6 \mathrm{H} 24}(13), v_{\mathrm{N} 3 \mathrm{C} 18}(13), v_{\mathrm{C} 6 \mathrm{C} 7}(9), v_{\mathrm{C} 8 \mathrm{C} 16}(5)$ \\
\hline 75 & 1610.6 & 27.4 & 10.8 & $\begin{array}{l}v_{\mathrm{C} 13 \mathrm{C} 15}(15), v_{\mathrm{C} 10 \mathrm{C} 11}(14), v_{\mathrm{O} 5 \mathrm{C} 9}(14), v_{\mathrm{C} 12 \mathrm{C} 13}(8),{ }^{3} \beta_{3 / R 6}(7) \\
v_{\mathrm{C}_{10 \mathrm{C} 14}}(6), \rho_{\mathrm{C} 13 \mathrm{H} 27}(6)\end{array}$ \\
\hline 76 & 1618.6 & 51.0 & 17.9 & $\rho_{\mathrm{N} 3 \mathrm{H} 23}(17), v_{\mathrm{C} 8 \mathrm{C} 19}(12), v_{\mathrm{C} 7 \mathrm{C} 18}(8), v_{\mathrm{C} 6 \mathrm{C} 19}(7), v_{\mathrm{C} 16 \mathrm{C} 18}(6), v_{\mathrm{C}_{10} \mathrm{C}_{11}}(5)$ \\
\hline 77 & 1628.3 & 54.8 & 10.1 & $\begin{array}{l}v_{\mathrm{C} 14 \mathrm{C} 15}(18), v_{\mathrm{C}_{11} \mathrm{C} 12}(14), v_{\mathrm{C} 12 \mathrm{C} 13}(11),{ }^{3} \beta_{2 / R \sigma}(9), v_{\mathrm{C} 10 \mathrm{C} 14}(7) \\
\rho_{\mathrm{N} 3 \mathrm{H} 23}(6), \rho_{\mathrm{C} 12 \mathrm{H} 26}(5)\end{array}$ \\
\hline 78 & 1632.7 & 303.5 & 3.5 & $v_{\mathrm{C} 6 \mathrm{C} 7}(20), \rho_{\mathrm{N} 3 \mathrm{H} 23}(13), v_{\mathrm{N} 3 \mathrm{C} 18}(8), \rho_{\mathrm{C} 7 \mathrm{H} 25}(7),{ }^{1} \beta_{3 / R 6}(7), v_{\mathrm{C} 8 \mathrm{C} 19}(7)$ \\
\hline 79 & 1667.0 & 181.9 & 26.6 & $v_{\mathrm{O} 5 \mathrm{C} 9}(41),{ }^{2} \beta_{3 / R \sigma}(7),{ }^{2} \beta_{\text {trig/R6}}(6)$ \\
\hline 80 & 1720.0 & 180.9 & 29.9 & $v_{\mathrm{O} 4 \mathrm{C} 17}(70),{ }^{2} \beta_{\text {trig/R6 }}(9)$ \\
\hline 81 & 2672.1 & 1.9 & 6.7 & $v_{\mathrm{S} 1 \mathrm{H} 33}(100)$ \\
\hline 82 & 3003.8 & 50.7 & 12.5 & $v_{\mathrm{C}_{2} \mathrm{H} 22}(95)$ \\
\hline 83 & 3056.7 & 31.0 & 11.3 & $v_{\mathrm{C}_{20} \mathrm{H} 32}(50), v_{\mathrm{C} 20 \mathrm{H} 31}(48)$ \\
\hline 84 & 3099.2 & 2.6 & 5.6 & $v_{\mathrm{C}_{22 \mathrm{H} 31}}(39), v_{\mathrm{C} 20 \mathrm{H} 32}(31), v_{\mathrm{C}_{2} \mathrm{H} 21}(28)$ \\
\hline 85 & 3116.3 & 19.4 & 2.9 & $v_{\mathrm{C}_{2} \mathrm{H} 21}(67), v_{\mathrm{C}_{20} \mathrm{H} 32}(18), v_{\mathrm{C} 20 \mathrm{H} 31}(12)$ \\
\hline 86 & 3170.1 & 3.5 & 4.4 & $v_{\mathrm{C} 15 \mathrm{H} 29}(50), v_{\mathrm{C} 13 \mathrm{H} 27}(44)$ \\
\hline 87 & 3173.6 & 11.4 & 7.7 & $v_{\mathrm{C} 6 \mathrm{H} 24}(92)$ \\
\hline 88 & 3184.9 & 13.8 & 14.5 & $v_{\mathrm{C} 13 \mathrm{H} 27}(44), v_{\mathrm{C} 15 \mathrm{H} 29}(42), v_{\mathrm{C} 12 \mathrm{H} 26}(9)$ \\
\hline 89 & 3202.2 & 3.8 & 6.7 & $v_{\mathrm{C} 12 \mathrm{H} 26}(82), v_{\mathrm{C} 13 \mathrm{H} 27}(9), v_{\mathrm{C} 14 \mathrm{H} 28}(8)$ \\
\hline 90 & 3206.4 & 9.1 & 11.2 & $v_{\mathrm{C} 14 \mathrm{H} 28}(84), v_{\mathrm{C}_{15 \mathrm{H}} 29}(7), v_{\mathrm{C} 12 \mathrm{H} 26}(6)$ \\
\hline 91 & 3211.8 & 0.2 & 4.1 & $v_{\mathrm{C} 19 \mathrm{H} 30}(75), v_{\mathrm{C} 7 \mathrm{H} 25}(24)$ \\
\hline 92 & 3214.8 & 8.0 & 16.1 & $v_{\mathrm{C} 7 \mathrm{H} 25}(71), v_{\mathrm{C} 19 \mathrm{H} 30}(21), v_{\mathrm{C} 6 \mathrm{H} 24}(6)$ \\
\hline 93 & 3426.2 & 114.0 & 34.5 & $v_{\mathrm{N} 3 \mathrm{H} 23}(99)$ \\
\hline
\end{tabular}


Table S.9. Frequencies of normal vibrations, IR and Raman intensities and PEDs for $A c-G-G$ conformer calculated at the B3LYP/6-311++G(d,p) level of theory.

\begin{tabular}{|c|c|c|c|c|}
\hline $\mathbf{N r}$ & $\begin{array}{l}\text { Freq. } \\
{\left[\mathrm{cm}^{-1}\right]}\end{array}$ & IR Int. & $\begin{array}{c}\text { Raman } \\
\text { Int. }\end{array}$ & PED $($ contributions $>5 \%)$ \\
\hline 1 & 18.2 & 0.7 & 14.6 & $\tau_{\mathrm{N} 3 \mathrm{C} 18}(46), \omega_{\mathrm{N} 3 \mathrm{H} 23}(39)$ \\
\hline 2 & 37.2 & 0.7 & 4.3 & $\tau_{\mathrm{C} 2 \mathrm{~N} 3}(27),{ }^{2} \tau_{3 / R 6}(23),{ }^{1} \tau_{3 / R 6}(17), \tau_{\mathrm{N} 3 \mathrm{C} 18}(14), \omega_{\mathrm{N} 3 \mathrm{H} 23}(7)$ \\
\hline 3 & 45.4 & 4.5 & 1.7 & ${ }^{2} \tau_{3 / R \sigma}(33), \tau_{\mathrm{C} 2 \mathrm{~N} 3}(28),{ }^{2} \tau_{2 / R \sigma}(17)$ \\
\hline 4 & 69.7 & 0.2 & 0.5 & $\begin{array}{l}{ }^{2} \tau_{2 / R \sigma}(22),{ }^{1} \tau_{3 / R \sigma}(14), \tau_{\mathrm{C}_{2} \mathrm{C} 20}(12), \omega_{\mathrm{N} 3 \mathrm{H} 23}(11),{ }^{2} \tau_{3 / R}(6), \tau_{\mathrm{C} 2 \mathrm{~N} 3}(5), \\
{ }^{1} \tau_{2 / R \sigma}(5),{ }^{1} \tau_{1 / R 6}(5)\end{array}$ \\
\hline 5 & 106.1 & 1.9 & 0.4 & $\tau_{\mathrm{C}_{2} \mathrm{C} 20}(26),{ }^{2} \tau_{2 / R 6}(16), \omega_{\mathrm{N} 3 \mathrm{H} 23}(16), \beta_{\mathrm{C} 18 \mathrm{~N} 3 \mathrm{C} 2_{2}}(9), \tau_{\mathrm{N} 3 \mathrm{C} 18}(7),{ }^{3} \tau_{2 / R 6}(5)$ \\
\hline 6 & 121.0 & 1.0 & 0.7 & ${ }^{2} \tau_{1 / R \sigma}(40),{ }^{3} \tau_{2 / R \sigma}(14),{ }^{2} \tau_{2 / R \sigma}(8),{ }^{3} \tau_{3 / R \sigma}(7)$ \\
\hline 7 & 154.4 & 0.5 & 0.7 & $\delta_{2 / \mathrm{C} 20 \mathrm{H} 31 \mathrm{H} 32}(21), \omega_{\mathrm{N} 3 \mathrm{H} 23}(16), \rho_{\mathrm{C} 18 \mathrm{~N} 3}(9), \delta_{2 / \mathrm{C}_{2} \mathrm{H} 22 \mathrm{H} 21}(9),{ }^{2} \tau_{1 / R 6}(8)$ \\
\hline 8 & 165.3 & 0.7 & 0.0 & $\begin{array}{l}{ }^{2} b f l_{R R}(23),{ }^{4} b f l_{R R}(21),{ }^{2} \tau_{3 / R 6}(9), \tau_{N 3 C l 8}(9),{ }^{1} \tau_{3 / R 6}(7), \omega_{C l 7 O 4}(6), \\
\omega_{C l 9 H 30}(5)\end{array}$ \\
\hline 9 & 191.1 & 5.4 & 1.0 & ${ }^{1} \tau_{2 / R \sigma}(21),{ }^{1} \tau_{3 / R \sigma}(18), \tau_{\mathrm{S} 1 \mathrm{C} 20}(14),{ }^{4} b f l_{R R}(14)$ \\
\hline 10 & 204.6 & 13.9 & 0.2 & $\tau_{\mathrm{S} 1 \mathrm{C} 20}(48),{ }^{\mathrm{l}} \tau_{3 / R 6}(9),{ }^{\mathrm{I}} \tau_{2 / R 6}(7), \delta_{2 / \mathrm{C}_{2} \mathrm{H} 22 \mathrm{H} 21}(6)$ \\
\hline 11 & 240.2 & 4.4 & 0.3 & $\tau_{\mathrm{S}_{1} \mathrm{C} 20}(15), \delta_{2 / \mathrm{C} 20 \mathrm{H} 31 \mathrm{H} 32}(11),{ }^{3} \beta_{2 / R 6}(6), \delta_{2 / \mathrm{C} 2 \mathrm{H} 22 \mathrm{H} 21}(6),{ }^{2} b f l_{R R}(6)$ \\
\hline 12 & 261.2 & 0.8 & 0.5 & $\begin{array}{l}{ }^{2} b f l_{R R}(27), \omega_{C l 8 N 3}(11), \omega_{N 3 H 23}(7),{ }^{4} b f l_{R R}(6), \delta_{2 / C 20 H 31 H 32}(5), \\
{ }^{1} \tau_{2 / R 6}(5)\end{array}$ \\
\hline 13 & 303.1 & 2.2 & 0.7 & ${ }^{2} \beta_{2 / R 6}(32), \delta_{2 / \mathrm{C}_{20} \mathrm{H} 31 \mathrm{H} 32}(20), v_{\mathrm{C} 8 \mathrm{C} 17}(6)$ \\
\hline 14 & 322.4 & 1.5 & 0.7 & $\beta_{\mathrm{C}_{18 \mathrm{~N} 3} \mathrm{C}_{2}}(18), \delta_{2 / \mathrm{C}_{2} \mathrm{H} 22 \mathrm{H} 21}(13), \tau_{\mathrm{C}_{2} \mathrm{C}_{20}}(8), \tau_{\mathrm{C}_{2} \mathrm{~N} 3}(7)$ \\
\hline 15 & 350.0 & 0.6 & 0.0 & ${ }^{2} \beta_{3 / R 6}(26), \rho_{\mathrm{C} 18 \mathrm{~N} 3}(15), \rho_{\mathrm{C} 17 \mathrm{O} 4}(13),{ }^{2} \beta_{2 / R 6}(8), v_{\mathrm{C} 8 \mathrm{C} 17}(5)$ \\
\hline 16 & 369.7 & 9.6 & 1.2 & ${ }^{2} \beta_{3 / R 6}(17),{ }^{1} \beta_{3 / R 6}(13), \rho_{\mathrm{C} 17 \mathrm{O} 4}(9), v_{\mathrm{C} 11 \mathrm{C} 17}(9), v_{\mathrm{C} 9 \mathrm{C} 16}(8), \rho$ \\
\hline 17 & 426.9 & 0.1 & 0.0 & ${ }^{3} \tau_{3 / R 6}(59),{ }^{4} b f l_{R R}(14),{ }^{3} \tau_{2 / R \sigma}(6)$ \\
\hline 18 & 430.2 & 21.2 & 0.6 & $\rho_{\mathrm{C} 9 \mathrm{O} 5}(41), \rho_{\mathrm{C} 17 \mathrm{O} 4}(16), v_{\mathrm{C} 9 \mathrm{C} 16}(9),{ }^{\mathrm{I}} \beta_{3 / R 6}(9)$ \\
\hline 19 & 433.1 & 0.1 & 0.1 & ${ }^{3} \tau_{2 / R \sigma}(31),{ }^{1} \tau_{2 / R \sigma}(20),{ }^{1} \tau_{3 / R \sigma}(17),{ }^{2} \tau_{1 / R 6}(7), \omega_{\mathrm{C} 9 \mathrm{O} 5}(6)$ \\
\hline 20 & 448.2 & 1.3 & 0.5 & $\begin{array}{l}{ }^{1} \beta_{2 / R 6}(16),{ }^{2} \beta_{2 / R 6}(13), \delta_{2 / \mathrm{C}_{2} \mathrm{H}_{22} \mathrm{H} 21}(13),{ }^{2} \beta_{3 / R \sigma}(11),{ }^{3} \beta_{2 / R 6}(8), \\
\delta_{2 / \mathrm{C} 20 \mathrm{H} 31 \mathrm{H} 32}(7)\end{array}$ \\
\hline 21 & 477.4 & 3.0 & 9.0 & $\begin{array}{l}{ }^{2} \beta_{2 / R \sigma}(28),{ }^{2} \beta_{3 / R \sigma}(21),{ }^{3} \beta_{3 / R \sigma}(9),{ }^{1} \beta_{3 / R \sigma}(8), v_{\mathrm{C} 8 \mathrm{C} 16}(6), v_{\mathrm{C} 10 \mathrm{C} 11}(5), \\
v_{\mathrm{C} 11 \mathrm{C} 17}(5)\end{array}$ \\
\hline 22 & 490.0 & 0.9 & 0.1 & ${ }^{3} \tau_{2 / R \sigma}(23),{ }^{1} \tau_{3 / R \sigma}(22),{ }^{1} \tau_{2 / R \sigma}(11),{ }^{2} \tau_{2 / R 6}(9),{ }^{3} \tau_{3 / R \sigma}(6),{ }^{1} \tau_{1 / R \sigma}(5)$ \\
\hline 23 & 507.1 & 2.2 & 1.5 & $\begin{array}{l}\delta_{2 / \mathrm{C} 2 \mathrm{H} 22 \mathrm{H} 21}(23), \rho_{\mathrm{C}_{20 \mathrm{H} 31 \mathrm{H} 32}(10),} \rho_{\mathrm{C} 18 \mathrm{~N} 3}(9),{ }^{1} \beta_{2 / R 6}(8),{ }^{3} \beta_{2 / R 6}(5), \\
\delta_{2 / \mathrm{C} 20 \mathrm{H} 31 \mathrm{H} 32}(5), \beta_{\mathrm{C}_{18 \mathrm{~N} 3 \mathrm{C} 2}(5)}\end{array}$ \\
\hline 24 & 551.7 & 6.3 & 0.2 & $\omega_{\mathrm{C} 18 \mathrm{~N} 3}(32),{ }^{1} \tau_{3 / R \sigma}(14),{ }^{1} \tau_{2 / R \sigma}(13),{ }^{1} \tau_{1 / R 6}(8),{ }^{2} b f l_{R R}(6)$ \\
\hline 25 & 601.9 & 4.9 & 0.6 & $\begin{array}{l}{ }^{1} \beta_{3 / R 6}(17),{ }^{1} \beta_{2 / R 6}(12),{ }^{3} \beta_{2 / R 6}(11), \beta_{\mathrm{C} 18 \mathrm{~N} 3 \mathrm{C} 2_{2}}(11), \rho_{\mathrm{C} 18 \mathrm{~N} 3}(7), \\
{ }^{3} \beta_{3 / R 6}(7)\end{array}$ \\
\hline 26 & 630.7 & 1.7 & 0.1 & ${ }^{2} \beta_{\text {trig/R6}}(41),{ }^{1} \beta_{2 / R 6}(15), \rho_{\mathrm{C} 18 \mathrm{~N} 3}(6), v_{\mathrm{S} 1 \mathrm{C} 20}(5)$ \\
\hline 27 & 647.6 & 6.4 & 1.7 & $v_{\mathrm{S}_{1} \mathrm{C}_{20}}(63), \delta_{2 / \mathrm{C}_{20} \mathrm{H}_{31} \mathrm{H}_{32}}(5)$ \\
\hline 28 & 669.3 & 1.5 & 0.1 & ${ }^{3} \tau_{1 / R 6}(38),{ }^{1} \tau_{1 / R 6}(19), \omega_{\mathrm{C} 9 \mathrm{O} 5}(12), \omega_{\mathrm{C} 18 \mathrm{~N} 3}(12)$ \\
\hline 29 & 677.0 & 11.4 & 1.2 & ${ }^{3} \beta_{3 / R \sigma}(40),{ }^{3} \beta_{2 / R 6}(9), v_{\mathrm{C} 10 \mathrm{C} 11}(7)$ \\
\hline 30 & 705.7 & 1.2 & 0.5 & $\rho_{\mathrm{C} 9 \mathrm{O} 5}(20),{ }^{3} \beta_{2 / R 6}(19), \rho_{\mathrm{C} 17 \mathrm{O} 4}(18),{ }^{1} \beta_{2 / R 6}(11),{ }^{1} \beta_{3 / R 6}(7)$ \\
\hline 31 & 715.9 & 60.2 & 0.2 & ${ }^{1} \tau_{1 / R 6}(29), \omega_{\mathrm{N}_{3} \mathrm{H} 23}(12),{ }^{3} \tau_{1 / R 6}(12), \tau_{\mathrm{N} 3 \mathrm{C} 18}(12), \omega_{\mathrm{C} 6 \mathrm{H} 24}(10), \tau_{\mathrm{C}_{2 N} 3}(7)$ \\
\hline 32 & 726.7 & 12.3 & 0.0 & $\begin{array}{l}{ }^{3} \tau_{1 / R 6}(21), \omega_{\mathrm{C} 17 \mathrm{O} 4}(11), \omega_{\mathrm{C}_{15} \mathrm{H} 29}(11),{ }^{1} \tau_{1 / R 6}(10), \omega_{\mathrm{C} 9 \mathrm{O} 5}(8), \\
\omega_{\mathrm{C}_{12} \mathrm{H} 26}(6), \omega_{\mathrm{N} 3 \mathrm{H} 23}(6), \tau_{\mathrm{N} 3 \mathrm{C} 18}(5)\end{array}$ \\
\hline 33 & 752.0 & 97.3 & 0.0 & $\begin{array}{l}\omega_{\mathrm{C} 18 \mathrm{~N} 3}(13), \omega_{\mathrm{C} 13 \mathrm{H} 27}(12),{ }^{1} \tau_{1 / R 6}(12),{ }^{3} \tau_{1 / R 6}(9), \omega_{\mathrm{N} 3 \mathrm{H} 23}(7), \\
\omega_{\mathrm{C} 9 \mathrm{O} 5}(6), \omega_{\mathrm{C} 17 \mathrm{O} 4}(6), \omega_{\mathrm{C} 14 \mathrm{H} 28}(6), \omega_{\mathrm{C} 15 \mathrm{H} 29}(5)\end{array}$ \\
\hline 34 & 774.5 & 7.0 & 1.2 & $\rho_{\mathrm{C} 20 \mathrm{H} 31 \mathrm{H} 32}(32), \beta_{\mathrm{C}_{20} \mathrm{~S} 1 \mathrm{H} 33}(21),{ }_{3 / R 6}(7)$ \\
\hline 35 & 786.4 & 3.6 & 0.0 & $\begin{array}{l}{ }^{3} \tau_{1 / R 6}(17), \omega_{\mathrm{C} 7 \mathrm{H} 25}(16), \omega_{\mathrm{C} 19 \mathrm{H} 30}(11),{ }^{2} \tau_{1 / R 6}(11), \omega_{\mathrm{C} 6 \mathrm{H} 24}(10), \\
\omega_{\mathrm{C} 17 \mathrm{O} 4}(8), \omega_{\mathrm{C} 955}(8), \omega_{\mathrm{C} 15 \mathrm{H} 29}(5)\end{array}$ \\
\hline
\end{tabular}




\begin{tabular}{|c|c|c|c|c|}
\hline 36 & 797.7 & 1.0 & 0.5 & $\begin{array}{l}{ }^{1} \beta_{\text {trig/R6 }}(15),{ }^{3} \beta_{2 / R 6}(10),{ }^{1} \beta_{3 / R 6}(10), \rho_{\mathrm{C} 20 \mathrm{H} 31 \mathrm{H} 32}(8),{ }^{3} \beta_{\text {trig/R6}}(8), \\
\beta_{\mathrm{C} 20 \mathrm{~S} 1 \mathrm{H} 33}(8),{ }^{2} \beta_{\text {trig/R6 }}(6), v_{\mathrm{C} 9 \mathrm{C} 10}(5)\end{array}$ \\
\hline 37 & 809.0 & 0.2 & 0.1 & $\begin{array}{l}\omega_{\mathrm{C} 9 \mathrm{O} 5}(27),{ }^{1} \tau_{1 / R 6}(12), \omega_{\mathrm{C}_{13} \mathrm{H} 27}(12),{ }^{2} \tau_{1 / R 6}(9), \omega_{\mathrm{C} 18 \mathrm{~N} 3}(9) \\
\omega_{\mathrm{C} 15 \mathrm{H} 29}(6)\end{array}$ \\
\hline 38 & 825.2 & 23.3 & 0.0 & $\begin{array}{l}\omega_{\mathrm{C} 7 \mathrm{H} 25}(21), \omega_{\mathrm{C} 17 \mathrm{O} 4}(20),{ }^{1} \tau_{1 / R 6}(14), \omega_{\mathrm{C} 15 \mathrm{H} 29}(8), \omega_{\mathrm{C} 18 \mathrm{~N} 3}(7), \\
{ }^{2} \tau_{1 / R 6}(7), \omega_{\mathrm{C} 6 \mathrm{H} 24}(5)\end{array}$ \\
\hline 39 & 869.6 & 15.7 & 1.8 & $\begin{array}{l}{ }^{1} \beta_{2 / R 6}(8), v_{\mathrm{C} 16 \mathrm{C} 18}(8), \rho_{\mathrm{C} 17 \mathrm{O} 4}(7), \beta_{\mathrm{C} 20 \mathrm{~S} 1 \mathrm{H} 33}(7), v_{\mathrm{C} 7 \mathrm{C} 18}(7), v_{\mathrm{N} 3 \mathrm{C} 18}(6), \\
{ }^{2} \beta_{\text {trig/R6 }}(6), v_{\mathrm{C} 2 \mathrm{~N} 3}(6)\end{array}$ \\
\hline 40 & 911.0 & 0.7 & 0.0 & $\omega_{\mathrm{C} 19 \mathrm{H} 30}(30), \omega_{\mathrm{C} 7 \mathrm{H} 25}(18), \omega_{\mathrm{C} 14 \mathrm{H} 28}(13), \omega_{\mathrm{C} 12 \mathrm{H} 26}(13), \omega_{\mathrm{C} 15 \mathrm{H} 29}(7)$ \\
\hline 41 & 917.9 & 0.4 & 0.0 & $\begin{array}{l}\omega_{\mathrm{C} 19 \mathrm{H} 30}(19), \omega_{\mathrm{C} 14 \mathrm{H} 28}(17), \omega_{\mathrm{C} 12 \mathrm{H} 26}(16), \omega_{\mathrm{C} 7 \mathrm{H} 25}(11), \omega_{\mathrm{C} 13 \mathrm{H} 27}(9), \\
\omega_{\mathrm{C} 15 \mathrm{H} 29}(7)\end{array}$ \\
\hline 42 & 924.3 & 3.1 & 0.3 & $\begin{array}{l}\beta_{\mathrm{C} 20 \mathrm{~S} 1 \mathrm{H} 33_{3}}(21), \rho_{\mathrm{C} 2 \mathrm{H} 22 \mathrm{H} 21}(11), v_{\mathrm{C} 2 \mathrm{C} 20}(7), \rho_{\mathrm{C} 9 \mathrm{O} 5}(7), \rho_{\mathrm{C} 20 \mathrm{H} 31 \mathrm{H} 32}(6), \\
\tau_{\mathrm{C} 20 \mathrm{H} 31 \mathrm{H} 32}(6), v_{\mathrm{C} 16 \mathrm{C} 18}(5)\end{array}$ \\
\hline 43 & 930.4 & 1.5 & 1.3 & ${ }^{1} \beta_{\text {trig/R6}}(21),{ }^{3} \beta_{\text {trig/R6 }}(20), \rho_{\mathrm{C} 9 \mathrm{O} 5}(9), \rho_{\mathrm{C} 17 \mathrm{O} 4}(6), \beta_{\mathrm{C}_{20} \mathrm{~S}_{1 \mathrm{H} 33}(6)}$ \\
\hline 44 & 942.9 & 19.4 & 0.4 & $v_{\mathrm{C}_{2} \mathrm{C} 20}(28), \rho_{\mathrm{C}_{2} \mathrm{H} 22 \mathrm{H} 21}(28), v_{\mathrm{S}_{1} \mathrm{C}_{20}}(8), \delta_{2 / \mathrm{C}_{2} \mathrm{H} 22 \mathrm{H} 21}(6)$ \\
\hline 45 & 1000.1 & 0.5 & 0.0 & $\omega_{\mathrm{C} 6 \mathrm{H} 24}(48), \omega_{\mathrm{C} 19 \mathrm{H} 30}(19), \omega_{\mathrm{C} 7 \mathrm{H} 25}(10),{ }^{1} \tau_{1 / R 6}(7)$ \\
\hline 46 & 1002.7 & 2.3 & .0 & $\omega_{\mathrm{C} 12 \mathrm{H} 26}(31), \omega_{\mathrm{C} 14 \mathrm{H} 28}(28), \omega_{\mathrm{C} 13 \mathrm{H} 27}(10), \omega_{\mathrm{C} 15 \mathrm{H} 29}(9), \omega_{\mathrm{C} 6 \mathrm{H} 24}(7)$ \\
\hline 47 & 1012.3 & 70.3 & 0.9 & $\begin{array}{l}v_{\mathrm{C} 2 \mathrm{C} 20}(16), v_{\mathrm{C} 8 \mathrm{C} 17}(13), v_{\mathrm{C} 2 \mathrm{~N} 3}(8), v_{\mathrm{C} 11 \mathrm{C} 12}(7), \rho_{\mathrm{C} 17 \mathrm{O} 4}(6), v_{\mathrm{C} 13 \mathrm{C} 15}(5), \\
v_{\mathrm{C} 8 \mathrm{C} 19}(5)\end{array}$ \\
\hline 48 & 1016.3 & 0.0 & 0 & $\omega_{\mathrm{C} 15 \mathrm{H} 29}(30), \omega_{\mathrm{C} 13 \mathrm{H} 27}(28), \omega_{\mathrm{C} 14 \mathrm{H} 28}(15), \omega_{\mathrm{C} 12 \mathrm{H} 26}(13),{ }^{3} \tau_{1 / R 6}(12)$ \\
\hline 49 & 1057.1 & 2.8 & 6.1 & $v_{\mathrm{C} 13 \mathrm{C} 15}(33), v_{\mathrm{C} 14 \mathrm{C} 15}(13), \rho_{\mathrm{C} 14 \mathrm{H} 28}(11), \rho_{\mathrm{C} 12 \mathrm{H} 26}(11), v_{\mathrm{C} 12 \mathrm{C} 13}(10)$ \\
\hline 50 & 1079.9 & 38.1 & 1.9 & $\begin{array}{l}\rho_{\mathrm{C} 2 \mathrm{H} 22 \mathrm{H} 21}(22), \beta_{\mathrm{C}_{20} \mathrm{~S}_{1} \mathrm{H} 33}(14), \rho_{\mathrm{C} 20 \mathrm{H} 31 \mathrm{H} 32}(12), v_{\mathrm{C} 2 \mathrm{C} 20}(11), v_{\mathrm{C} 7 \mathrm{C} 18}(7), \\
v_{\mathrm{C} 6 \mathrm{C} 19}(6)\end{array}$ \\
\hline 51 & 1100.1 & 8.9 & 0.4 & $\begin{array}{l}v_{\mathrm{C} 6 \mathrm{C} 19}(24),{ }^{1} \beta_{\text {trig/R6 }}(14), \rho_{\mathrm{C} 19 \mathrm{H} 30}(13), v_{\mathrm{C} 2 \mathrm{~N} 3}(8), v_{\mathrm{C} 2 \mathrm{C} 20}(7), \\
\rho_{\mathrm{C} 2 \mathrm{H} 22 \mathrm{H} 21}(6)\end{array}$ \\
\hline 52 & 1113.3 & 1.2 & 0.3 & $\begin{array}{l}{ }^{3} \beta_{\text {trig/R6 }}(37), v_{\mathrm{C} 12 \mathrm{C} 13}(8), v_{\mathrm{C} 14 \mathrm{C} 15}(8), \rho_{\mathrm{C} 13 \mathrm{H} 27}(8), \rho_{\mathrm{C} 15 \mathrm{H} 29}(7), \\
v_{\mathrm{C} 9 \mathrm{C} 10}(6), \rho_{\mathrm{C} 14 \mathrm{H} 28}(5), v_{\mathrm{C} 11 \mathrm{C} 17}(5)\end{array}$ \\
\hline 53 & 1135.8 & 3.7 & 3.4 & $v_{\mathrm{C}_{2} \mathrm{~N} 3}(37), \tau_{\mathrm{C}_{20} \mathrm{H} 31 \mathrm{H} 32}(7), \rho_{\mathrm{C} 18 \mathrm{~N} 3}(7), v_{\mathrm{C} 8 \mathrm{C} 17}(6)$ \\
\hline 54 & 1175.9 & 5.9 & 3.7 & $\begin{array}{l}\rho_{\mathrm{C} 13 \mathrm{H} 27}(17), \rho_{\mathrm{C} 12 \mathrm{H} 26}(12), v_{\mathrm{C} 9 \mathrm{C} 16}(9), v_{\mathrm{C} 9 \mathrm{C} 10}(7),{ }^{1} \beta_{\text {trig/R6 }}(7), \\
{ }^{3} \beta_{\text {trig/R6}}(6)\end{array}$ \\
\hline 55 & 1183.8 & 22.2 & 0.4 & $\begin{array}{l}\rho_{\mathrm{C} 15 \mathrm{H} 29}(31), \rho_{\mathrm{C} 14 \mathrm{H} 28}(10), v_{\mathrm{C} 14 \mathrm{C} 15}(9), \rho_{\mathrm{C} 13 \mathrm{H} 27}(8), v_{\mathrm{C} 13 \mathrm{C} 15}(5), \\
{ }^{1} \beta_{\text {trig/R6}}(5),{ }^{3} \beta_{\text {trig/R6}}(5)\end{array}$ \\
\hline 56 & 1198.7 & 25.7 & 7.6 & $\rho_{\mathrm{C} 6 \mathrm{H} 24}(30), \rho_{\mathrm{C} 7 \mathrm{H} 25}(13), v_{\mathrm{C} 6 \mathrm{C} 7}(7), \rho_{\mathrm{C} 13 \mathrm{H} 27}(6)$ \\
\hline 57 & 1215.5 & 1.9 & 4.2 & $\rho_{\mathrm{C} 19 \mathrm{H} 30}(26), \rho_{\mathrm{C} 7 \mathrm{H} 25}(11), v_{\mathrm{C} 8 \mathrm{C} 19}(9), v_{\mathrm{C} 11 \mathrm{C} 17}(8), \rho_{\mathrm{C} 6 \mathrm{H} 24}(6), \rho_{\mathrm{C} 14 \mathrm{H} 28}(6)$ \\
\hline 58 & 1233.4 & 64.5 & 0.9 & $\tau_{\mathrm{C} 20 \mathrm{H} 31 \mathrm{H} 32}(45), \tau_{\mathrm{C} 2 \mathrm{H} 22 \mathrm{H} 21}(8), v_{\mathrm{C}_{2} \mathrm{~N} 3}(7)$ \\
\hline 59 & 1254.8 & 81.0 & 5.7 & $\omega_{\mathrm{C} 20 \mathrm{H} 31 \mathrm{H} 32}(23), \tau_{\mathrm{C} 2 \mathrm{H} 22 \mathrm{H} 21}(16), \tau_{\mathrm{C} 20 \mathrm{H} 31 \mathrm{H} 32}(12), v_{\mathrm{C} 7 \mathrm{C} 18}(7)$ \\
\hline 60 & 1270.1 & 26.9 & 0.5 & $\begin{array}{l}\tau_{\mathrm{C} 2 \mathrm{H} 22 \mathrm{H} 21}(15), \rho_{\mathrm{C} 12 \mathrm{H} 26}(13), \rho_{\mathrm{C} 14 \mathrm{H} 28}(11),{ }^{2} \beta_{\text {trig/R6 }}(10) \\
\omega_{\mathrm{C} 20 \mathrm{H} 31 \mathrm{H} 32}(10), v_{\mathrm{C} 11 \mathrm{C} 12}(6)\end{array}$ \\
\hline 61 & 1291.9 & 557.5 & 10.7 & $\begin{array}{l}v_{\mathrm{C} 9 \mathrm{C} 10}(13), v_{\mathrm{C} 9 \mathrm{C} 16}(9), v_{\mathrm{C} 8 \mathrm{C} 16}(7), v_{\mathrm{C} 11 \mathrm{C} 17}(6), \omega_{\mathrm{C} 20 \mathrm{H} 31 \mathrm{H} 32}(6), \\
v_{\mathrm{C} 7 \mathrm{C} 18}(5), v_{\mathrm{C} 8 \mathrm{C} 17}(5), \rho_{\mathrm{C} 14 \mathrm{H} 28}(5), \rho_{\mathrm{C} 19 \mathrm{H} 30}(5), v_{\mathrm{C} 16 \mathrm{C} 18}(5)\end{array}$ \\
\hline 62 & 1318.5 & 141.4 & 1.3 & $v_{\mathrm{C} 11 \mathrm{C} 17}(19), v_{\mathrm{C} 8 \mathrm{C} 17}(14), \rho_{\mathrm{C} 12 \mathrm{H} 26}(8), \rho_{\mathrm{C} 17 \mathrm{O} 4}(7),{ }^{2} \beta_{2 / R 6}(7), \rho_{\mathrm{C} 13 \mathrm{H} 27}(5)$ \\
\hline 63 & 1327.2 & 145.2 & 5.5 & $\begin{array}{l}\omega_{\mathrm{C} 20 \mathrm{H} 31 \mathrm{H} 32}(25), v_{\mathrm{N} 3 \mathrm{C} 18}(18), \tau_{\mathrm{C} 2 \mathrm{H} 22 \mathrm{H} 21}(9), v_{\mathrm{C} 16 \mathrm{C} 18}(8), \rho_{\mathrm{N} 3 \mathrm{H} 23}(6), \\
\omega_{\mathrm{C} 2 \mathrm{H} 22 \mathrm{H} 21}(6)\end{array}$ \\
\hline 64 & 1351.5 & 69.0 & 0.9 & $\begin{array}{l}\omega_{\mathrm{C} 20 \mathrm{H} 31 \mathrm{H} 32}(18), \tau_{\mathrm{C} 2 \mathrm{H} 22 \mathrm{H} 21}(16), v_{\mathrm{C} 16 \mathrm{C} 18}(8), v_{\mathrm{C} 9 \mathrm{C} 16}(8), \rho_{\mathrm{N}_{3} \mathrm{H} 23}(8), \\
v_{\mathrm{N}_{3} \mathrm{C} 18}(5)\end{array}$ \\
\hline 65 & 1358.7 & 27.7 & 1.1 & $\begin{array}{l}v_{\mathrm{C} 10 \mathrm{C} 14}(14), v_{\mathrm{C} 11 \mathrm{C} 12}(14), v_{\mathrm{C} 10 \mathrm{C} 11}(14), v_{\mathrm{C} 13 \mathrm{C} 15}(13), v_{\mathrm{C} 12 \mathrm{C} 13}(13) \\
v_{\mathrm{C} 14 \mathrm{C} 15}(12)\end{array}$ \\
\hline 66 & 1381.6 & 12.2 & 1.6 & $\begin{array}{l}v_{\mathrm{C} 16 \mathrm{C} 18}(14), v_{\mathrm{C} 8 \mathrm{C} 16}(14), v_{\mathrm{C} 8 \mathrm{C} 19}(11), v_{\mathrm{C} 6 \mathrm{C} 19}(7), v_{\mathrm{C} 9 \mathrm{C} 16}(6), v_{\mathrm{C} 6 \mathrm{C} 7}(5), \\
v_{\mathrm{C} 7 \mathrm{C} 18}(5)\end{array}$ \\
\hline
\end{tabular}




\begin{tabular}{|c|c|c|c|c|}
\hline 67 & 1392.9 & 15.9 & 2.2 & $\omega_{\mathrm{C} 2 \mathrm{H} 22 \mathrm{H} 21}(70)$ \\
\hline 68 & 1435.5 & 38.7 & 0.5 & $\rho_{\mathrm{C} 7 \mathrm{H} 25}(26), v_{\mathrm{C} 8 \mathrm{C} 19}(12), v_{\mathrm{C} 6 \mathrm{C} 7}(12), v_{\mathrm{C} 8 \mathrm{C} 16}(7), v_{\mathrm{C} 9 \mathrm{C} 16}(6)$ \\
\hline 69 & 1462.1 & 10.3 & 0.8 & $\delta_{1 / \mathrm{C}_{20} \mathrm{H} 31 \mathrm{H} 32}(91)$ \\
\hline 70 & 1478.3 & 0.3 & 4.0 & $\begin{array}{l}\rho_{\mathrm{C} 15 \mathrm{H} 29}(15), \rho_{\mathrm{C} 13 \mathrm{H} 27}(13), v_{\mathrm{C} 10 \mathrm{C} 14}(7), \rho_{\mathrm{C} 19 \mathrm{H} 30}(7), v_{\mathrm{C} 11 \mathrm{C} 12}(6), \\
v_{\mathrm{C} 8 \mathrm{C} 16}(6), v_{\mathrm{O} 5 \mathrm{C} 9}(5)\end{array}$ \\
\hline 71 & 1488.6 & 8.6 & 0.9 & $\begin{array}{l}\rho_{\mathrm{C} 19 \mathrm{H} 30}(11), v_{\mathrm{C} 8 \mathrm{C} 16}(8), \rho_{\mathrm{C} 13 \mathrm{H} 27}(7), \delta_{1 / \mathrm{C}_{2 \mathrm{H}} 2 \mathrm{H} 21}(7), \rho_{\mathrm{C} 15 \mathrm{H} 29}(6), \\
\rho_{\mathrm{C} 6 \mathrm{H} 24}(6), v_{\mathrm{C} 8 \mathrm{C} 17}(5)\end{array}$ \\
\hline 72 & 1504.3 & 4.5 & 1.4 & $\delta_{1 / \mathrm{C}_{2} \mathrm{H} 22 \mathrm{H} 21}(77)$ \\
\hline 73 & 1508.1 & 2.1 & 1.7 & $\rho_{\mathrm{C} 14 \mathrm{H} 28}(19), \rho_{\mathrm{C} 12 \mathrm{H} 26}(18), v_{\mathrm{C} 10 \mathrm{C} 11}(16), v_{\mathrm{C} 13 \mathrm{C} 15}(8)$ \\
\hline 74 & 1559.7 & 158.4 & 1.5 & $v_{\mathrm{C} 6 \mathrm{C} 19}(21), \rho_{\mathrm{C} 6 \mathrm{H} 24}(14), v_{\mathrm{N}_{3} \mathrm{C} 18}(13), \rho_{\mathrm{N} 3 \mathrm{H} 23}(12), v_{\mathrm{C} 6 \mathrm{C} 7}(10)$ \\
\hline 75 & 1610.2 & 26.5 & 11.1 & $\begin{array}{l}v_{\mathrm{C} 13 \mathrm{C} 15}(15), v_{\mathrm{O} 5 \mathrm{C} 9}(15), v_{\mathrm{C} 10 \mathrm{C} 11}(14), v_{\mathrm{C} 12 \mathrm{C} 13}(8),{ }^{3} \beta_{3 / R 6}(6), \\
v_{\mathrm{C} 10 \mathrm{C} 14}(6), \rho_{\mathrm{C} 13 \mathrm{H} 27}(6)\end{array}$ \\
\hline 76 & 1619.2 & 42.1 & 20.2 & $\rho_{\mathrm{N} 3 \mathrm{H} 23}(15), v_{\mathrm{C} 8 \mathrm{C} 19}(13), v_{\mathrm{C} 7 \mathrm{C} 18}(8), v_{\mathrm{C} 6 \mathrm{C} 19}(7), v_{\mathrm{C} 16 \mathrm{C} 18}(6), v_{\mathrm{C} 10 \mathrm{C} 11}(5)$ \\
\hline 77 & 1628.7 & 68.3 & 9.0 & $\begin{array}{l}v_{\mathrm{C} 14 \mathrm{C} 15}(18), v_{\mathrm{C} 11 \mathrm{C} 12}(14), v_{\mathrm{C} 12 \mathrm{C} 13}(11),{ }^{3} \beta_{2 / R 6}(9), v_{\mathrm{C} 10 \mathrm{C} 14}(7), \\
\rho_{\mathrm{C} 12 \mathrm{H} 26}(5), \rho_{\mathrm{C} 14 \mathrm{H} 28}(5), \rho_{\mathrm{N} 3 \mathrm{H} 23}(5)\end{array}$ \\
\hline 78 & 1634.9 & 323.0 & 3.4 & $v_{\mathrm{C} 6 \mathrm{C} 7}(19), \rho_{\mathrm{N} 3 \mathrm{H} 23}(19), v_{\mathrm{N} 3 \mathrm{C} 18}(10), \rho_{\mathrm{C} 7 \mathrm{H} 25}(6),{ }^{1} \beta_{3 / R 6}(6), v_{\mathrm{C} 8 \mathrm{C} 19}(5)$ \\
\hline 79 & 1666.4 & 190.9 & 25.2 & $v_{\mathrm{O} 5 \mathrm{C} 9}(39),{ }^{2} \beta_{3 / R 6}(6),{ }^{2} \beta_{\text {trig/R6 }}(6)$ \\
\hline 80 & 1719.3 & 179.0 & 29.7 & $v_{\mathrm{O} 4 \mathrm{C} 17}(70),{ }^{2} \beta_{\text {trig/R6 }}(9)$ \\
\hline 81 & 2658.1 & 5.0 & 7.3 & $v_{\mathrm{S} 1 \mathrm{H} 33}(100)$ \\
\hline 82 & 3004.0 & 51.2 & 12.0 & $v_{\mathrm{C} 2 \mathrm{H} 22}(95)$ \\
\hline 83 & 3057.1 & 34.4 & 12.8 & $v_{\mathrm{C}_{20 \mathrm{H} 31}}(58), v_{\mathrm{C} 20 \mathrm{H} 32}(40)$ \\
\hline 84 & 3094.4 & 5.6 & 3.9 & $v_{\mathrm{C} 2 \mathrm{H} 21}(63), v_{\mathrm{C} 20 \mathrm{H} 31}(17), v_{\mathrm{C}_{20} \mathrm{H} 32}(15)$ \\
\hline 85 & 3112.0 & 22.3 & 4.3 & $v_{\mathrm{C}_{20} \mathrm{H} 32}(44), v_{\mathrm{C}_{2} \mathrm{H}_{21}}(31), v_{\mathrm{C}_{20} \mathrm{H} 31}(23)$ \\
\hline 86 & 3169.6 & 3.6 & 4.4 & $v_{\mathrm{C} 15 \mathrm{H} 29}(50), v_{\mathrm{C} 13 \mathrm{H} 27}(44)$ \\
\hline 87 & 3172.3 & 12.1 & 7.7 & $v_{\mathrm{C} 6 \mathrm{H} 24}(93)$ \\
\hline 88 & 3184.5 & 14.5 & 14.5 & $v_{\mathrm{C}_{13} \mathrm{H} 27}(44), v_{\mathrm{C} 15 \mathrm{H} 29}(42), v_{\mathrm{C} 12 \mathrm{H} 26}(8)$ \\
\hline 89 & 3202.1 & 3.9 & 6.5 & $v_{\mathrm{C} 12 \mathrm{H} 26}(82), v_{\mathrm{C} 14 \mathrm{H} 28}(9), v_{\mathrm{C} 13 \mathrm{H} 27}(8)$ \\
\hline 90 & 3206.3 & 9.3 & 11.0 & $v_{\mathrm{C} 14 \mathrm{H} 28}(84), v_{\mathrm{C} 15 \mathrm{H} 29}(7), v_{\mathrm{C} 12 \mathrm{H} 26}(6)$ \\
\hline 91 & 3212.4 & 0.3 & 5.7 & $v_{\mathrm{C} 19 \mathrm{H} 30}(90), v_{\mathrm{C} 7 \mathrm{H} 25}(8)$ \\
\hline 92 & 3217.5 & 16.6 & 14.2 & $v_{\mathrm{C} 7 \mathrm{H} 25}(88), v_{\mathrm{C} 19 \mathrm{H} 30}(6)$ \\
\hline 93 & 3423.0 & 114.0 & 35.0 & $v_{\mathrm{N} 3 \mathrm{H} 23}(99)$ \\
\hline
\end{tabular}


Table S.10. Frequencies of normal vibrations, IR and Raman intensities and PEDs for ScGT conformer calculated at the B3LYP/6-311++G(d,p) level of theory.

\begin{tabular}{|c|c|c|c|c|}
\hline $\mathbf{N r}$ & $\begin{array}{l}\text { Freq. } \\
{\left[\mathrm{cm}^{-1}\right]}\end{array}$ & IR Int. & $\begin{array}{c}\text { Raman } \\
\text { Int. }\end{array}$ & PED $($ contributions $>5 \%)$ \\
\hline 1 & 23.7 & 1.9 & 10.2 & $\tau_{\mathrm{N} 3 \mathrm{C} 18}(46), \omega_{\mathrm{N} 3 \mathrm{H} 23}(32),{ }^{2} \tau_{3 / R 6}(7)$ \\
\hline 2 & 31.8 & 3.0 & 7.3 & ${ }^{2} \tau_{3 / R 6}(27), \tau_{\mathrm{C}_{2} \mathrm{~N} 3}(26),{ }^{1} \tau_{3 / R 6}(17), \tau_{\mathrm{C}_{2} \mathrm{C} 20}(8)$ \\
\hline 3 & 50.4 & 1.3 & 1.7 & ${ }^{2} \tau_{3 / R 6}(26), \tau_{\mathrm{C}_{2} \mathrm{~N} 3}(17), \omega_{\mathrm{N} 3 \mathrm{H} 23}(15),{ }^{2} \tau_{2 / R \sigma}(10), \tau_{\mathrm{C}_{2} \mathrm{C} 20}(9), \tau_{\mathrm{S}_{1} \mathrm{C}_{20}}(6)$ \\
\hline 4 & 59.7 & 5.8 & 1.3 & $\tau_{\mathrm{C}_{2} \mathrm{C} 20}(33), \tau_{\mathrm{S} 1 \mathrm{C} 20}(20),{ }^{2} \tau_{2 / R 6}(13),{ }^{1} \tau_{3 / R 6}(7), \tau_{\mathrm{N} 3 \mathrm{C} 18}(5)$ \\
\hline 5 & 83.9 & 10.9 & 1.2 & $\tau_{\mathrm{S}_{1} \mathrm{C} 20}(33), \omega_{\mathrm{N} 3 \mathrm{H} 23}(20),{ }^{2} \tau_{2 / R 6}(11), \tau_{\mathrm{C}_{2} \mathrm{C} 20}(9)$ \\
\hline 6 & 117.5 & 1.2 & 0.8 & ${ }^{2} \tau_{1 / R \sigma}(35),{ }^{3} \tau_{2 / R \sigma}(17),{ }^{2} \tau_{2 / R 6}(16),{ }^{3} \tau_{3 / R 6}(8)$ \\
\hline 7 & 134.8 & 2.5 & 0.3 & $\tau_{\mathrm{S}_{1 \mathrm{C} 20}}(37),{ }^{2} \tau_{1 / R 6}(11), \omega_{\mathrm{N} 3 \mathrm{H} 23}(9), \beta_{\mathrm{C} 18 \mathrm{~N} 3 \mathrm{C} 2_{2}}(9), \tau_{\mathrm{C}_{2} \mathrm{C} 20}(6), \rho_{\mathrm{C} 18 \mathrm{~N} 3}(6)$ \\
\hline 8 & 160.4 & 0.2 & 0.6 & $\begin{array}{l}\delta_{2 / \mathrm{C} 20 \mathrm{H} 31 \mathrm{H} 32}(15),{ }^{\mathrm{i}} \tau_{2 / R 6}(11), \rho_{\mathrm{C} 18 \mathrm{~N} 3}(8), \beta_{\mathrm{C}_{18 \mathrm{~N} 3 \mathrm{C} 2}}(7),{ }^{4} b f l_{R R}(6), \\
{ }_{\tau_{3 / R 6}}(6), \tau_{N 3 \mathrm{Cl}}(5)\end{array}$ \\
\hline 9 & 168.7 & 0.6 & 0.4 & $\begin{array}{l}{ }^{4} b f l_{R R}(23),{ }^{2} b f l_{R R}(20), \tau_{N 3 C l 8}(7), \delta_{2 / C 20 H 31 H 32}(6), \omega_{C 9 O 5}(6), \\
{ }^{2} \tau_{2 / R 6}(6)\end{array}$ \\
\hline 10 & 203.8 & 0.9 & 0.9 & ${ }^{1}{ }^{1}{ }_{3 / R \sigma}(24),{ }^{1} \tau_{2 / R 6}(20),{ }^{4} b f l_{R R}(10), \omega_{C 19 H 30}(6)$ \\
\hline 11 & 215.4 & 0.3 & 0.2 & $\begin{array}{l}\delta_{2 / \mathrm{C}_{20 \mathrm{H} 31 \mathrm{H} 32}}(15), \delta_{2 / \mathrm{C}_{2} \mathrm{H} 22 \mathrm{H} 21}(14),{ }^{4} b f l_{R R}(10),{ }^{2} b f l_{R R}(7), \beta_{C 18 N 3 C_{2}}(7), \\
{ }^{{ }_{\tau_{3 / R 6}}(5)}\end{array}$ \\
\hline 12 & 266.4 & 0.9 & 0.6 & $\begin{array}{l}{ }^{2} b f l_{R R}(23), \delta_{2 / C 20 H 3 l H 32}(8), \omega_{C 18 N 3}(8),{ }^{l} \tau_{2 / R 6}(6),{ }^{l} \tau_{3 / R 6}(5), \\
\omega_{C 7 H 25}(5), \omega_{N 3 H 23}(5)\end{array}$ \\
\hline 13 & 282.6 & 2.6 & 0.5 & ${ }^{2} \beta_{2 / R 6}(21), \rho_{\mathrm{C} 18 \mathrm{~N} 3}(13), v_{\mathrm{C} 8 \mathrm{C} 17}(7),{ }^{2} b f l_{R R}(6)$ \\
\hline 14 & 343.4 & 1.1 & 0.2 & ${ }^{2} \beta_{3 / R 6}(27), \rho_{\mathrm{C} 17 \mathrm{O} 4}(18), v_{\mathrm{C} 8 \mathrm{C} 17}(9), \rho_{\mathrm{C} 18 \mathrm{~N} 3}(5)$ \\
\hline 15 & 355.6 & 2.5 & 0.1 & $\begin{array}{l}{ }^{2} \beta_{2 / R 6}(24), \delta_{2 / \mathrm{C}_{20} \mathrm{H} 31 \mathrm{H} 32}(14), \beta_{\mathrm{C} 18 \mathrm{~N} 3 \mathrm{C} 2}(13), \rho_{\mathrm{C}_{18 \mathrm{~N} 3}}(11), \tau_{\mathrm{C}_{2 \mathrm{~N}} 3}(8), \\
\tau_{\mathrm{C}_{2} \mathrm{C} 20}(7)\end{array}$ \\
\hline 16 & 372.8 & 7.4 & 1.1 & ${ }^{2} \beta_{3 / R 6}(23),{ }^{1} \beta_{3 / R 6}(15), v_{\mathrm{C} 11 \mathrm{C} 17}(11), v_{\mathrm{C} 9 \mathrm{C} 16}(8), \rho_{\mathrm{C} 17 \mathrm{O} 4}(7),{ }^{3} \beta_{3 / R 6}(5)$ \\
\hline 17 & 424.8 & 7.4 & 0.3 & ${ }^{3} \tau_{3 / R 6}(41),{ }^{4} b f l_{R R}(10), \rho_{C 9 O 5}(9),{ }^{3} \tau_{2 / R 6}(5)$ \\
\hline 18 & 427.7 & 12.9 & 0.3 & ${ }^{3} \tau_{3 / R 6}(38), \rho_{\mathrm{C} 9 \mathrm{O} 5}(14),{ }^{4} b f l_{R R}(10), \rho_{C_{17 O 4}}(6)$ \\
\hline 19 & 432.3 & 0.0 & 0.0 & ${ }^{3} \tau_{2 / R 6}(30),{ }^{1} \tau_{2 / R 6}(21),{ }^{1} \tau_{3 / R 6}(17),{ }^{2} \tau_{1 / R 6}(7), \omega_{\mathrm{C} 9 \mathrm{O} 5}(6)$ \\
\hline 20 & 445.7 & 6.8 & 0.1 & $\delta_{2 / \mathrm{C}_{2} \mathrm{H} 22 \mathrm{H} 21}(19),{ }^{2} \beta_{2 / R 6}(16), \delta_{2 / \mathrm{C}_{20} \mathrm{H} 31 \mathrm{H} 32}(7),{ }^{1} \beta_{2 / R 6}(7)$ \\
\hline 21 & 477.1 & 1.4 & 9.9 & ${ }^{2} \beta_{3 / R 6}(33),{ }^{1} \beta_{3 / R 6}(12),{ }^{2} \beta_{2 / R 6}(12),{ }^{1} \beta_{2 / R 6}(7),{ }^{3} \beta_{3 / R 6}(6), v_{\mathrm{C} 10 \mathrm{C} 11}(5)$ \\
\hline 22 & 489.8 & 1.5 & 0.0 & ${ }^{3} \tau_{2 / R \sigma}(23),{ }^{1} \tau_{3 / R \sigma}(21),{ }^{1} \tau_{2 / R \sigma}(12),{ }^{2} \tau_{2 / R \sigma}(9),{ }^{3} \tau_{3 / R \sigma}(6),{ }^{1} \tau_{1 / R \sigma}(6)$ \\
\hline 23 & 500.8 & 5.7 & 1.3 & $\delta_{2 / \mathrm{C}_{2} \mathrm{H}_{22} \mathrm{H} 21}(20),{ }^{1} \beta_{2 / R 6}(12),{ }^{2} \beta_{2 / R 6}(11), \rho_{\mathrm{C}_{20} \mathrm{H} 31 \mathrm{H} 32}(5)$ \\
\hline 24 & 557.4 & 5.4 & 0.1 & $\omega_{\mathrm{C} 18 \mathrm{~N} 3}(29),{ }^{1} \tau_{3 / R \sigma}(14),{ }^{1} \tau_{2 / R \sigma}(12),{ }^{1} \tau_{1 / R 6}(10),{ }^{2} b f l_{R R}(6)$ \\
\hline 25 & 602.6 & 3.9 & 0.4 & $\begin{array}{l}{ }^{1} \beta_{3 / R \sigma}(13),{ }^{3} \beta_{2 / R 6}(13), \beta_{\mathrm{C} 18 \mathrm{~N}_{3} \mathrm{C} 2}(11),{ }^{1} \beta_{2 / R 6}(10), \rho_{\mathrm{C} 18 \mathrm{~N} 3}(7), \\
{ }^{2} \beta_{\text {trig/R6}}(6),{ }^{3} \beta_{3 / R 6}(5)\end{array}$ \\
\hline 26 & 631.2 & 2.3 & 0.3 & ${ }^{2} \beta_{\text {trig/R6}}(39),{ }^{1} \beta_{2 / R 6}(15), v_{\mathrm{S} 1 \mathrm{C} 20_{0}}(6), \rho_{\mathrm{C} 18 \mathrm{~N} 3}(6), \beta_{\mathrm{C} 18 \mathrm{~N} 3 \mathrm{C} 2}(5)$ \\
\hline 27 & 662.8 & 2.9 & 0.8 & $v_{\mathrm{S} 1 \mathrm{C} 20}(48),{ }^{3} \beta_{3 / R 6}(14),{ }^{3} \beta_{2 / R 6}(6)$ \\
\hline 28 & 667.2 & 12.2 & 0.1 & ${ }^{3} \tau_{1 / R 6}(31), \omega_{\mathrm{C} 9 \mathrm{O} 5}(11), \omega_{\mathrm{N} 3 \mathrm{H} 23}(10), \omega_{\mathrm{C} 18 \mathrm{~N} 3}(9), \tau_{\mathrm{N} 3 \mathrm{C} 18}(8),{ }^{1} \tau_{1 / R 6}(7)$ \\
\hline 29 & 674.5 & 22.4 & 1.2 & ${ }^{1} \tau_{1 / R 6}(14),{ }^{3} \tau_{1 / R 6}(13),{ }^{3} \beta_{3 / R 6}(13), \tau_{\mathrm{N} 3 \mathrm{C} 18}(9), v_{\mathrm{S}_{1 \mathrm{C} 20}}(6)$ \\
\hline 30 & 685.5 & 40.1 & 1.4 & $\begin{array}{l}{ }^{3} \beta_{3 / R 6}(15), \tau_{\mathrm{N} 3 \mathrm{C} 18}(11), v_{\mathrm{S}_{1} \mathrm{C} 20}(8), \tau_{\mathrm{C}_{2} \mathrm{~N} 3}(7), \omega_{\mathrm{N}_{3} \mathrm{H}_{23}}(7), \beta_{\mathrm{C} 18 \mathrm{~N} 3 \mathrm{C} 2}(6), \\
{ }^{1} \tau_{1 / R 6}(5),{ }^{1} \beta_{3 / R 6}(5)\end{array}$ \\
\hline 31 & 708.9 & 5.7 & 0.9 & ${ }^{3} \beta_{2 / R 6}(19), \rho_{\mathrm{C} 9 \mathrm{O} 5}(17), \rho_{\mathrm{C} 17 \mathrm{O} 4}(16),{ }^{1} \beta_{2 / R 6}(9),{ }^{1} \beta_{3 / R 6}(5), \rho_{\mathrm{C} 18 \mathrm{~N} 3}(5)$ \\
\hline 32 & 724.7 & 32.8 & 0.0 & ${ }^{3} \tau_{1 / R 6}(28),{ }^{1} \tau_{1 / R 6}(26), \omega_{\mathrm{C} 6 \mathrm{H} 24}(8), \omega_{\mathrm{C} 15 \mathrm{H} 29}(7), \omega_{\mathrm{C} 17 \mathrm{O} 4}(7)$ \\
\hline 33 & 747.1 & 61.8 & 0.1 & $\begin{array}{l}{ }^{\mathrm{l}} \tau_{1 / R 6}(14), \omega_{\mathrm{C} 13 \mathrm{H} 27}(14), \omega_{\mathrm{C} 18 \mathrm{~N} 3}(13), \omega_{\mathrm{C} 9 \mathrm{O} 5}(9), \omega_{\mathrm{C} 17 \mathrm{O} 4}(8), \\
{ }^{3} \tau_{1 / R 6}(7), \omega_{\mathrm{C} 14 \mathrm{H} 28}(7), \omega_{\mathrm{C}_{15} \mathrm{H} 29}(7)\end{array}$ \\
\hline 34 & 782.9 & 4.9 & 2.2 & $\begin{array}{l}{ }^{1} \beta_{3 / R 6}(14),{ }^{1} \beta_{\text {trig/R6 }}(9), \rho_{\mathrm{C} 20 \mathrm{H} 31 \mathrm{H} 32}(9),{ }^{3} \beta_{2 / R 6}(8), \beta_{\mathrm{C}_{20 \mathrm{~S} 1 \mathrm{H} 33}}(7), \\
\rho_{\mathrm{C} 2 \mathrm{H} 22 \mathrm{H} 21}(6)\end{array}$ \\
\hline 35 & 785.4 & 6.0 & 0.4 & $\begin{array}{l}{ }^{3} \tau_{1 / R 6}(18), \omega_{\mathrm{C} 7 \mathrm{H} 25}(13),{ }^{2} \tau_{1 / R 6}(12), \omega_{\mathrm{C} 9 \mathrm{O} 5}(10), \omega_{\mathrm{C} 19 \mathrm{H} 30}(9), \\
\omega_{\mathrm{C} 6 \mathrm{H} 24}(9), \omega_{\mathrm{C} 17 \mathrm{O} 4}(9)\end{array}$ \\
\hline
\end{tabular}




\begin{tabular}{|c|c|c|c|c|}
\hline 36 & 807.3 & 1.5 & 0.3 & \begin{tabular}{|l}
$\rho_{\mathrm{C} 20 \mathrm{H} 31 \mathrm{H} 32}(16), \beta_{\mathrm{C} 20 \mathrm{~S} 1 \mathrm{H} 33}(15),{ }^{1} \beta_{\text {trig/R6}}(8), v_{\mathrm{C} 2 \mathrm{C} 20}(7), \rho_{\mathrm{C}_{2} \mathrm{H} 22 \mathrm{H} 21}(6)$, \\
${ }^{1} \beta_{2 / R 6}(6),{ }^{2} \beta_{\text {trig/R6 }}(5),{ }^{3} \beta_{\text {trig/R6 }}(5)$
\end{tabular} \\
\hline 37 & 809.3 & 0.7 & 0.1 & $\begin{array}{l}\omega_{\mathrm{C} 955}(25), \omega_{\mathrm{C} 13 \mathrm{H} 27}(13),{ }^{1} \tau_{1 / R 6}(12), \omega_{\mathrm{C} 18 \mathrm{~N} 3}(9),{ }^{2} \tau_{1 / R 6}(7) \\
\omega_{\mathrm{C} 15 \mathrm{H} 29}(7)\end{array}$ \\
\hline 38 & 824.7 & 26.4 & 0.0 & $\begin{array}{l}\omega_{\mathrm{C} 7 \mathrm{H} 25}(22), \omega_{\mathrm{C} 17 \mathrm{O} 4}(18),{ }^{1} \tau_{1 / R 6}(16), \omega_{\mathrm{C} 18 \mathrm{~N} 3}(9),{ }^{2} \tau_{1 / R 6}(7), \\
\omega_{\mathrm{C} 15 \mathrm{H} 29}(7)\end{array}$ \\
\hline 39 & 875.2 & 17.9 & 2.5 & 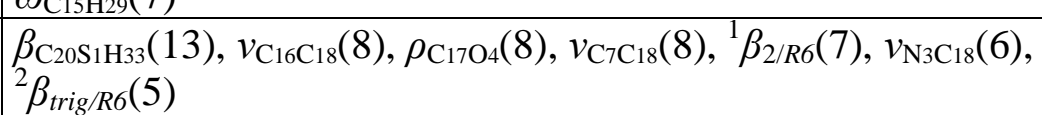 \\
\hline 40 & 910.0 & 1.0 & 0.0 & $\omega_{\mathrm{C} 19 \mathrm{H} 30}(38), \omega_{\mathrm{C} 7 \mathrm{H} 25}(26), \omega_{\mathrm{C} 12 \mathrm{H} 26}(8), \omega_{\mathrm{C} 14 \mathrm{H} 28}(7)$ \\
\hline 41 & 917.5 & 0.4 & 0.0 & $\begin{array}{l}\omega_{\mathrm{C} 14 \mathrm{H} 28}(23), \omega_{\mathrm{C} 12 \mathrm{H} 26}(21), \omega_{\mathrm{C} 13 \mathrm{H} 27}(11), \omega_{\mathrm{C} 19 \mathrm{H} 30}(11), \omega_{\mathrm{C} 15 \mathrm{H} 29}(9), \\
\omega_{\mathrm{C} 7 \mathrm{H} 25}(7)\end{array}$ \\
\hline 42 & 928.5 & 3.6 & 1.2 & ${ }^{1} \beta_{\text {trig/R6}}(24),{ }^{3} \beta_{\text {trig/R6 }}(23), \rho_{\mathrm{C} 9 \mathrm{O} 5}(15), v_{\mathrm{C} 16 \mathrm{C} 18}(8)$ \\
\hline 43 & 944.4 & 8.2 & 0.4 & $\beta_{\mathrm{C}_{20 \mathrm{~S} 1 \mathrm{H} 33}}(43), \rho_{\mathrm{C}_{20} \mathrm{H}_{31} \mathrm{H}_{32}}(18), v_{\mathrm{C}_{2} \mathrm{~N}_{3}}(6)$ \\
\hline 44 & 958.6 & 5.8 & 0.4 & $v_{\mathrm{C} 2 \mathrm{C} 20}(42), \rho_{\mathrm{C} 2 \mathrm{H} 22 \mathrm{H} 21}(15), \delta_{2 / \mathrm{C} 2 \mathrm{H} 22 \mathrm{H} 21}(11), \rho_{\mathrm{C} 20 \mathrm{H} 31 \mathrm{H} 32}(10), v_{\mathrm{S}_{1} \mathrm{C} 20}(8)$ \\
\hline 45 & 997.8 & 2.4 & 0.1 & $\omega_{\mathrm{C} 6 \mathrm{H} 24}(51), \omega_{\mathrm{C} 19 \mathrm{H} 30}(24), \omega_{\mathrm{C} 7 \mathrm{H} 25}(9),{ }^{1} \tau_{1 / R 6}(7)$ \\
\hline 46 & 1002.3 & 67.4 & 1.0 & $\rho_{\mathrm{C} 20 \mathrm{H} 31 \mathrm{H} 32}(13), \rho_{\mathrm{C}_{2} \mathrm{H} 22 \mathrm{H} 21}(11), v_{\mathrm{C} 8 \mathrm{C} 17}(9), v_{\mathrm{C} 2 \mathrm{~N} 3}(9), v_{\mathrm{C}_{11} \mathrm{C}_{12}}(5)$ \\
\hline 47 & 1003.3 & 2.1 & 0.0 & $\omega_{\mathrm{C} 12 \mathrm{H} 26}(36), \omega_{\mathrm{C} 14 \mathrm{H} 28}(29), \omega_{\mathrm{C} 13 \mathrm{H} 27}(13), \omega_{\mathrm{C} 15 \mathrm{H} 29}(9),{ }^{3} \tau_{3 / R 6}(5)$ \\
\hline 48 & 1016.6 & 0.0 & 0.0 & $\omega_{\mathrm{C} 15 \mathrm{H} 29}(31), \omega_{\mathrm{C} 13 \mathrm{H} 27}(26), \omega_{\mathrm{C} 14 \mathrm{H} 28}(17), \omega_{\mathrm{C} 12 \mathrm{H} 26}(12),{ }^{3} \tau_{1 / R 6}(12)$ \\
\hline 49 & 1042.7 & 23.4 & 1.9 & $\begin{array}{l}\rho_{\mathrm{C} 2 \mathrm{H} 22 \mathrm{H} 21}(22), \tau_{\mathrm{C}_{2} \mathrm{H} 31 \mathrm{H} 32}(10), v_{\mathrm{C} 13 \mathrm{C} 15}(9), \rho_{\mathrm{C} 20 \mathrm{H} 31 \mathrm{H} 32}(6), v_{\mathrm{C} 7 \mathrm{C} 18}(6), \\
v_{\mathrm{C}_{2} \mathrm{C} 20}(6), \beta_{\mathrm{C} 20 \mathrm{~S} 1 \mathrm{H} 33}(5)\end{array}$ \\
\hline 50 & 1060.4 & 1.4 & .2 & $v_{\mathrm{C} 13 \mathrm{C} 15}(25), \rho_{\mathrm{C} 12 \mathrm{H} 26}(10), \rho_{\mathrm{C} 14 \mathrm{H} 28}(10), v_{\mathrm{C} 14 \mathrm{C} 15}(10), v_{\mathrm{C} 12 \mathrm{C} 13}(7)$ \\
\hline 51 & 1098.4 & 26.8 & 1.7 & $v_{\mathrm{C} 6 \mathrm{C} 19}(30), \rho_{\mathrm{C} 19 \mathrm{H} 30}(16),{ }^{1} \beta_{\text {trig/R6 }}(14), v_{\mathrm{C} 6 \mathrm{C} 7}(8), v_{\mathrm{C} 9 \mathrm{C} 16}(5)$ \\
\hline 52 & 1113.3 & 1.1 & 0.3 & $\begin{array}{l}{ }^{3} \beta_{\text {trig/R6}}(38), v_{\mathrm{C} 12 \mathrm{C} 13}(9), v_{\mathrm{C} 14 \mathrm{C} 15}(8), \rho_{\mathrm{C} 13 \mathrm{H} 27}(8), \rho_{\mathrm{C} 15 \mathrm{H} 29}(7), \\
v_{\mathrm{C} 9 \mathrm{C} 10}(6), v_{\mathrm{C} 11 \mathrm{C} 17}(5), \rho_{\mathrm{C} 14 \mathrm{H} 28}(5), \rho_{\mathrm{C} 12 \mathrm{H} 26}(5)\end{array}$ \\
\hline 53 & 1147.7 & 12.1 & 3.3 & $v_{\mathrm{C} 2 \mathrm{~N} 3}(41), v_{\mathrm{C} 2 \mathrm{C} 20}(12), v_{\mathrm{C} 8 \mathrm{C} 17}(5), \rho_{\mathrm{C} 18 \mathrm{~N} 3}(5)$ \\
\hline 54 & 1175.6 & 6.5 & 4.3 & $\begin{array}{l}\rho_{\mathrm{C} 13 \mathrm{H} 27}(15), \rho_{\mathrm{C} 12 \mathrm{H} 26}(12), v_{\mathrm{C} 9 \mathrm{C} 16}(9), v_{\mathrm{C} 9 \mathrm{C} 10}(7),{ }^{1} \beta_{\text {trig/R6 }}(7), \\
{ }^{3} \beta_{\text {trig/R6 }}(6), v_{\mathrm{C} 7 \mathrm{C} 18}(5)\end{array}$ \\
\hline 55 & 1183.5 & 14.4 & 0.2 & $\rho_{\mathrm{C} 15 \mathrm{H} 29}(30), \rho_{\mathrm{C} 14 \mathrm{H} 28}(10), \rho_{\mathrm{C} 13 \mathrm{H} 27}(10), v_{\mathrm{C} 14 \mathrm{C} 15}(8), v_{\mathrm{C} 13 \mathrm{C} 15}(6)$ \\
\hline 56 & 1194.2 & 8.9 & 6.6 & $\tau_{\mathrm{C} 20 \mathrm{H} 31 \mathrm{H} 32}(41), \rho_{\mathrm{C} 6 \mathrm{H} 24}(11)$ \\
\hline 57 & 1201.7 & 29.6 & 6 & $\tau_{\mathrm{C} 20 \mathrm{H} 31 \mathrm{H} 32}(22), \rho_{\mathrm{C} 6 \mathrm{H} 24}(20), \rho_{\mathrm{C} 7 \mathrm{H} 25}(15), v_{\mathrm{C} 6 \mathrm{C} 7}(5)$ \\
\hline 58 & & 2.0 & & $\rho_{\mathrm{C} 19 \mathrm{H} 30}(26), v_{\mathrm{C} 8 \mathrm{C} 19}(10), \rho_{\mathrm{C} 7 \mathrm{H} 25}(9), v_{\mathrm{C} 11 \mathrm{C} 17}(8), \rho_{\mathrm{C} 14 \mathrm{H} 28}(7)$ \\
\hline 59 & 1253.2 & 93.1 & 2.0 & $\omega_{\mathrm{C} 20 \mathrm{H} 31 \mathrm{H} 32}(12), v_{\mathrm{C} 7 \mathrm{C} 18}(10), \rho_{\mathrm{C} 12 \mathrm{H} 26}(10), \tau_{\mathrm{C}_{2} \mathrm{H} 22 \mathrm{H} 21}(8), v_{\mathrm{C} 9 \mathrm{C} 10}(7)$ \\
\hline 60 & 1276.2 & 58.0 & 0.3 & $\omega_{\mathrm{C} 20 \mathrm{H} 31 \mathrm{H} 32}(23), \tau_{\mathrm{C} 2 \mathrm{H} 22 \mathrm{H} 21}(13), \rho_{\mathrm{C} 14 \mathrm{H} 28}(10),{ }^{2} \beta_{\text {trig/R6}}(9), \rho_{\mathrm{C} 12 \mathrm{H} 26}(8)$ \\
\hline 61 & 1293.8 & 561.9 & 10.8 & $\begin{array}{l}v_{\mathrm{C} 9 \mathrm{C} 10}(12), \omega_{\mathrm{C} 20 \mathrm{H} 31 \mathrm{H} 32}(11), v_{\mathrm{C} 9 \mathrm{C} 16}(9), v_{\mathrm{C} 8 \mathrm{C} 16}(6), v_{\mathrm{C}_{11} \mathrm{C} 17}(6), \\
v_{\mathrm{C} 8 \mathrm{C} 17}(5)\end{array}$ \\
\hline 62 & 1317.5 & 115.6 & 1.8 & $v_{\mathrm{C} 11 \mathrm{C} 17}(18), v_{\mathrm{C} 8 \mathrm{C} 17}(14), \rho_{\mathrm{C} 12 \mathrm{H} 26}(8), \rho_{\mathrm{C} 17 \mathrm{O} 4}(7),{ }^{2} \beta_{2 / R 6}(6)$ \\
\hline 63 & 1334.5 & 148.9 & 3.6 & $\begin{array}{l}v_{\mathrm{N} 3 \mathrm{C} 18}(16), \rho_{\mathrm{N} 3 \mathrm{H} 23}(12), \tau_{\mathrm{C} 2 \mathrm{H} 22 \mathrm{H} 21}(10), v_{\mathrm{C} 16 \mathrm{C} 18}(9), \omega_{\mathrm{C} 2 \mathrm{H} 22 \mathrm{H} 21}(9), \\
\rho_{\mathrm{C} 6 \mathrm{H} 24}(7), v_{\mathrm{C} 9 \mathrm{C} 16}(6)\end{array}$ \\
\hline 64 & 1341.7 & 47.2 & 3.2 & $\tau_{\mathrm{C}_{2} \mathrm{H} 22 \mathrm{H} 21}(30), \omega_{\mathrm{C}_{20} \mathrm{H} 31 \mathrm{H} 32}(29), v_{\mathrm{N} 3 \mathrm{C} 18}(12),{ }^{1} \beta_{\text {trig/R6}}(5)$ \\
\hline 65 & 1358.8 & 14.1 & 0.9 & $\begin{array}{l}v_{\mathrm{C} 10 \mathrm{C} 11}(15), v_{\mathrm{C} 10 \mathrm{C} 14}(15), v_{\mathrm{C} 11 \mathrm{C} 12}(14), v_{\mathrm{C} 13 \mathrm{C} 15}(14), v_{\mathrm{C} 12 \mathrm{C} 13}(13), \\
v_{\mathrm{C} 14 \mathrm{C} 15}(13)\end{array}$ \\
\hline 66 & 1378.6 & 21.4 & 1.7 & $\begin{array}{l}v_{\mathrm{C} 16 \mathrm{C} 18}(18), v_{\mathrm{C} 8 \mathrm{C} 19}(11), v_{\mathrm{C} 8 \mathrm{C} 16}(10), v_{\mathrm{C} 9 \mathrm{C} 16}(9), v_{\mathrm{C} 6 \mathrm{C} 19}(7), v_{\mathrm{C} 6 \mathrm{C} 7}(6), \\
v_{\mathrm{C} 7 \mathrm{C} 18}(6), \rho_{\mathrm{C} 9 \mathrm{O} 5}(6), v_{\mathrm{C} 9 \mathrm{C} 10}(5)\end{array}$ \\
\hline 67 & 1398.6 & 37.3 & & $\omega_{\mathrm{C}_{2} \mathrm{H} 22 \mathrm{H} 21}(67)$ \\
\hline 68 & 1435.0 & 32.5 & 0.5 & $\rho_{\mathrm{C} 7 \mathrm{H} 25}(24), v_{\mathrm{C} 6 \mathrm{C} 7}(11), v_{\mathrm{C} 8 \mathrm{C} 19}(10), v_{\mathrm{C} 8 \mathrm{C} 16}(6), v_{\mathrm{C} 9 \mathrm{C} 16}(6)$ \\
\hline 69 & 1478.4 & 1.0 & 3.4 & $\begin{array}{l}\rho_{\mathrm{C} 15 \mathrm{H} 29}(13), \rho_{\mathrm{C} 13 \mathrm{H} 27}(11), \delta_{1 / \mathrm{C} 20 \mathrm{H} 31 \mathrm{H} 32}(10), \rho_{\mathrm{C} 19 \mathrm{H} 30}(7), v_{\mathrm{C} 10 \mathrm{C} 14}(6), \\
v_{\mathrm{C} 8 \mathrm{C} 16}(5), v_{\mathrm{C} 11 \mathrm{C} 12}(5)\end{array}$ \\
\hline 70 & 1481.5 & 8.4 & 0.9 & $\delta_{1 / \mathrm{C}_{20} \mathrm{H}_{31} \mathrm{H}_{32}}(81)$ \\
\hline 71 & 1487.3 & 10.2 & 0.8 & ${ }_{19 H 30}(8), \rho_{\mathrm{C} 13 \mathrm{H} 27}(7), \rho_{\mathrm{C} 6 \mathrm{H} 24}(7), \rho_{\mathrm{C} 15 \mathrm{H} 29}(6)$, \\
\hline
\end{tabular}




\begin{tabular}{|c|c|c|c|c|}
\hline & & & & $v_{\mathrm{C} 8 \mathrm{C} 16}(6)$ \\
\hline 72 & 1503.2 & 4.6 & 1.8 & $\delta_{1 / \mathrm{C}_{2} \mathrm{H} 22 \mathrm{H} 21}(60)$ \\
\hline 73 & 1508.7 & 3.4 & 1.4 & $\begin{array}{l}\rho_{\mathrm{C} 14 \mathrm{H} 28}(18), \rho_{\mathrm{C} 12 \mathrm{H} 26}(17), v_{\mathrm{C} 10 \mathrm{C} 11}(15), v_{\mathrm{C} 13 \mathrm{C} 15}(7), \delta_{1 / \mathrm{C} 2 \mathrm{H} 22 \mathrm{H} 21}(6), \\
\rho_{\mathrm{C} 15 \mathrm{H} 29}(5)\end{array}$ \\
\hline 74 & 1554.6 & 191.5 & 1.4 & $v_{\mathrm{C} 6 \mathrm{C} 19}(19), \rho_{\mathrm{N} 3 \mathrm{H} 23}(18), v_{\mathrm{N} 3 \mathrm{C} 18}(16), \rho_{\mathrm{C} 6 \mathrm{H} 24}(12), v_{\mathrm{C} 6 \mathrm{C} 7}(7), v_{\mathrm{C} 8 \mathrm{C} 16}(6)$ \\
\hline 75 & 1612.1 & 37.2 & 10.0 & $v_{\mathrm{C} 13 \mathrm{C} 15}(13), v_{\mathrm{C} 10 \mathrm{C} 11}(13), v_{\mathrm{O} 5 \mathrm{C} 9}(9), v_{\mathrm{C} 12 \mathrm{C} 13}(6), v_{\mathrm{C} 8 \mathrm{C} 19}(6),{ }^{3} \beta_{3 / R 6}(5)$ \\
\hline 76 & 1615.0 & 82.5 & 6.3 & $\begin{array}{l}\rho_{\mathrm{N}_{32 \mathrm{H} 23}}(19), v_{\mathrm{C} 6 \mathrm{C} 19}(8), v_{\mathrm{C} 8 \mathrm{C} 19}(7), v_{\mathrm{C} 16 \mathrm{C} 18}(7), v_{\mathrm{C} 10 \mathrm{C} 11}(7), v_{\mathrm{C} 7 \mathrm{C} 18}(7), \\
v_{\mathrm{C}_{13} \mathrm{C} 15}(7),{ }_{2 / R 6}(5)\end{array}$ \\
\hline 77 & 1627.4 & 34.0 & 15.0 & $v_{\mathrm{C} 14 \mathrm{C} 15}(14), v_{\mathrm{C} 11 \mathrm{C} 12}(11), v_{\mathrm{O} 5 \mathrm{C} 9}(8),{ }^{3} \beta_{2 / R 6}(7), v_{\mathrm{C}_{12} \mathrm{C} 13}(7), v_{\mathrm{C} 6 \mathrm{C} 7}(5)$ \\
\hline 78 & 1631.2 & 279.7 & 4.8 & $\begin{array}{l}v_{\mathrm{C} 6 \mathrm{C} 7}(16), v_{\mathrm{C} 8 \mathrm{C} 19}(6),{ }_{3} \beta_{3 / R 6}(6), v_{\mathrm{C}_{14} \mathrm{C}_{15}}(6), v_{\mathrm{O} 4 \mathrm{C} 17}(6), \rho_{\mathrm{C} 7 \mathrm{H} 25}(5), \\
v_{\mathrm{N} 3 \mathrm{C} 18}(5), v_{\mathrm{C} 12 \mathrm{C} 13}(5)\end{array}$ \\
\hline 79 & 1671.4 & 205.5 & 27.8 & $v_{\mathrm{O} 5 \mathrm{C} 9}(45),{ }^{2} \beta_{3 / R 6}(6),{ }^{2} \beta_{\text {trig/R6}}(6)$ \\
\hline 80 & 1720.5 & 181.3 & 27.5 & $v_{\mathrm{O} 4 \mathrm{C} 17}(71),{ }^{2} \beta_{\text {trig/R6 }}(9)$ \\
\hline 81 & 2672.8 & 6.6 & 15.4 & $v_{\mathrm{S}_{1 \mathrm{H} 33}}(100)$ \\
\hline 82 & 3023.4 & 44.4 & 7.4 & $v_{\mathrm{C} 2 \mathrm{H} 21}(88), v_{\mathrm{C}_{2} \mathrm{H} 22}(10)$ \\
\hline 83 & 3058.1 & 22.7 & 11.8 & $v_{\mathrm{C} 20 \mathrm{H} 32}(50), v_{\mathrm{C}_{20} \mathrm{H} 31}(45)$ \\
\hline 84 & 3082.2 & 8.5 & 4.4 & $v_{\mathrm{C}_{2} \mathrm{H} 22}(77), v_{\mathrm{C} 2 \mathrm{H} 21}(12), v_{\mathrm{C}_{20} \mathrm{H}_{32}}(10)$ \\
\hline 85 & 3112.4 & 21.6 & 4.1 & $v_{\mathrm{C} 20 \mathrm{H} 31}(54), v_{\mathrm{C}_{20} \mathrm{H} 32}(39), v_{\mathrm{C}_{2} \mathrm{H} 22}(6)$ \\
\hline 86 & 3169.9 & 3.5 & 4.5 & $v_{\mathrm{C} 15 \mathrm{H} 29}(49), v_{\mathrm{C} 13 \mathrm{H} 27}(45)$ \\
\hline 87 & 3171.7 & 11.7 & 7.9 & $v_{\mathrm{C} 6 \mathrm{H} 24}(90), v_{\mathrm{C} 7 \mathrm{H} 25}(6)$ \\
\hline 88 & 3184.8 & 13.9 & 14.7 & $v_{\mathrm{C} 13 \mathrm{H} 27}(43), v_{\mathrm{C} 15 \mathrm{H} 29}(43), v_{\mathrm{C} 12 \mathrm{H} 26}(9)$ \\
\hline 89 & 3201.9 & 4.0 & 7.0 & $v_{\mathrm{C} 12 \mathrm{H} 26}(82), v_{\mathrm{C} 13 \mathrm{H} 27}(9), v_{\mathrm{C} 14 \mathrm{H} 28}(8)$ \\
\hline 90 & 3205.3 & 12.4 & 7.5 & $v_{\mathrm{C} 7 \mathrm{H} 25}(90)$ \\
\hline 91 & 3206.3 & 8.0 & 12.7 & $v_{\mathrm{C} 14 \mathrm{H} 28}(84), v_{\mathrm{C} 15 \mathrm{H} 29}(7), v_{\mathrm{C} 12 \mathrm{H} 26}(6)$ \\
\hline 92 & 3213.1 & 4.4 & 12.7 & $v_{\mathrm{C} 19 \mathrm{H} 30}(92)$ \\
\hline 93 & 3459.4 & 105.3 & 31.1 & $v_{\mathrm{N} 3 \mathrm{H} 23}(99)$ \\
\hline
\end{tabular}


Table S.11. Frequencies of normal vibrations, IR and Raman intensities and PEDs for $S c G G$ conformer calculated at the B3LYP/6-311++G(d,p) level of theory.

\begin{tabular}{|c|c|c|c|c|}
\hline $\mathbf{N r}$ & $\begin{array}{l}\text { Freq. } \\
{\left[\mathrm{cm}^{-1}\right]}\end{array}$ & IR Int. & $\begin{array}{c}\text { Raman } \\
\text { Int. }\end{array}$ & PED $($ contributions $>5 \%)$ \\
\hline 1 & 24.8 & 0.6 & 12.2 & $\tau_{\mathrm{N} 3 \mathrm{C} 18}(46), \omega_{\mathrm{N} 3 \mathrm{H} 23}(28), \tau_{\mathrm{C} 2 \mathrm{~N} 3}(10), \tau_{\mathrm{C} 2 \mathrm{C} 20}(5)$ \\
\hline 2 & 30.9 & 3.0 & 5.6 & ${ }^{2} \tau_{3 / R 6}(36), \tau_{\mathrm{C} 2 \mathrm{~N} 3}(21),{ }^{1} \tau_{3 / R 6}(15),{ }^{2} \tau_{2 / R 6}(7)$ \\
\hline 3 & 51.5 & 2.2 & 2.0 & ${ }^{2} \tau_{3 / R 6}(31), \tau_{\mathrm{C}_{2} \mathrm{~N} 3}(23),{ }^{2} \tau_{2 / R 6}(17), \omega_{\mathrm{N}_{3} \mathrm{H} 23}(9)$ \\
\hline 4 & 64.5 & 0.5 & 0.5 & $\tau_{\mathrm{C}_{2} \mathrm{C} 20}(25),{ }^{2} \tau_{2 / R 6}(17),{ }^{1} \tau_{3 / R 6}(15), \tau_{\mathrm{N} 3 \mathrm{C} 18}(9),{ }^{2} \tau_{3 / R 6}(6),{ }^{1} \tau_{2 / R 6}(5)$ \\
\hline 5 & 100.5 & 1.8 & 0.5 & $\tau_{\mathrm{C}_{2} \mathrm{C} 20}(23),{ }^{2} \tau_{2 / R 6}(18), \omega_{\mathrm{N} 3 \mathrm{H} 23}(17), \beta_{\mathrm{C}_{18 \mathrm{~N} 3 \mathrm{C} 2}}(8), \tau_{\mathrm{N} 3 \mathrm{C} 18}(6)$ \\
\hline 6 & 119.4 & 0.7 & 0.9 & ${ }^{2} \tau_{1 / R 6}(43),{ }^{3} \tau_{2 / R 6}(15),{ }^{2} \tau_{2 / R 6}(8),{ }^{3} \tau_{3 / R 6}(8), \omega_{\mathrm{N}_{3} \mathrm{H}_{23}}(6)$ \\
\hline 7 & 162.7 & 0.4 & 0.3 & $\begin{array}{l}{ }^{4} b f l_{R R}(14), \tau_{N 3 C 18}(10),{ }^{2} b f l_{R R}(10),{ }^{1} \tau_{2 / R 6}(9), \delta_{2 / C 20 H 31 H 32}(7), \\
{ }^{l} \tau_{3 / R 6}(7),{ }^{2} \tau_{3 / R 6}(6), \omega_{C 19 H 30}(5)\end{array}$ \\
\hline 8 & 171.4 & 0.9 & 0.4 & ${ }^{4} b f l_{R R}(18),{ }^{2} b f l_{R R}(15), \delta_{2 / C 20 H 31 H 32}(9),{ }^{2} \tau_{2 / R 6}(6)$ \\
\hline 9 & 192.3 & 4.7 & 0.7 & $\tau_{\mathrm{S}_{1} \mathrm{C} 20}(38), \delta_{2 / \mathrm{C}_{2} \mathrm{H} 22 \mathrm{H} 21}(8),{ }^{1} \tau_{2 / R 6}(8),{ }^{1} \tau_{3 / R \sigma}(6)$ \\
\hline 10 & 205.0 & 9.9 & 0.6 & $\tau_{\mathrm{S}_{1 \mathrm{C} 20}}(25),{ }^{\mathrm{1}} \tau_{3 / R 6}(18),{ }^{1} \tau_{2 / R 6}(13),{ }^{4} b f l_{R R}(10)$ \\
\hline 11 & 221.4 & 5.2 & 0.4 & $\begin{array}{l}\tau_{\mathrm{S}_{1 \mathrm{C} 20}}(21), \delta_{2 / \mathrm{C}_{20} \mathrm{H} 31 \mathrm{H} 32}(12),{ }^{2} b f l_{R R}(8), \beta_{\mathrm{Cl}_{18 N 3 C 2}}(7),{ }^{4} b f l_{R R}(7), \\
\delta_{2 / \mathrm{C} 2 \mathrm{H} 22 \mathrm{H} 21}(6)\end{array}$ \\
\hline 12 & 267.3 & 1.7 & 0.5 & $\begin{array}{l}{ }^{2} b f l_{R R}(22), \delta_{2 / C 20 H 3 l H 32}(8), \omega_{C l 8 N 3}(8),{ }^{I} \tau_{2 / R 6}(6),{ }^{I} \tau_{3 / R 6}(5), \\
\omega_{N 3 H 23}(5)\end{array}$ \\
\hline 13 & 283.6 & 3.0 & 0.5 & ${ }^{2} \beta_{2 / R 6}(22), \rho_{\mathrm{C} 18 \mathrm{~N} 3}(13), v_{\mathrm{C} 8 \mathrm{C} 17}(7),{ }^{2} b f l_{R R}(6)$ \\
\hline 14 & 343.7 & 0.8 & 0.2 & ${ }^{2} \beta_{3 / R 6}(29), \rho_{\mathrm{C} 17 \mathrm{O} 4}(18), v_{\mathrm{C} 8 \mathrm{C} 17}(9), \rho_{\mathrm{C} 18 \mathrm{~N} 3}(7),{ }^{1} \beta_{2 / R 6}(5)$ \\
\hline 15 & 361.9 & 5.2 & 0.3 & $\begin{array}{l}{ }^{2} \beta_{2 / R 6}(23), \rho_{\mathrm{C} 18 \mathrm{~N} 3}(12), \beta_{\mathrm{C} 18 \mathrm{~N} 3 \mathrm{C} 2_{2}}(11), \delta_{2 / \mathrm{C} 20 \mathrm{H} 31 \mathrm{H} 32}(10), \tau_{\mathrm{C} 2 \mathrm{C} 20}(7), \\
v_{\mathrm{C} 9 \mathrm{C} 10}(5), \tau_{\mathrm{C}_{2} \mathrm{~N} 3}(5)\end{array}$ \\
\hline 16 & 375.6 & 4.6 & 0.9 & ${ }^{2} \beta_{3 / R 6}(20),{ }^{1} \beta_{3 / R 6}(14), v_{\mathrm{C}_{11 \mathrm{C} 17}}(10), \delta_{2 / \mathrm{C} 20 \mathrm{H} 31 \mathrm{H} 32}(7), v_{\mathrm{C} 9 \mathrm{C} 16}(6)$ \\
\hline 17 & 425.5 & 10.2 & 0.3 & ${ }^{3} \tau_{3 / R \sigma}(40), \rho_{\mathrm{C} 9 \mathrm{O} 5}(12),{ }^{4} b f l_{R R}(10),{ }^{3} \tau_{2 / R 6}(5)$ \\
\hline 18 & 426.9 & 10.9 & 0.3 & ${ }^{3} \tau_{3 / R 6}(43), \rho_{\mathrm{C} 9 \mathrm{O} 5}(12),{ }^{4} b f l_{R R}(11)$ \\
\hline 19 & 432.4 & 0.0 & 0.0 & ${ }^{3} \tau_{2 / R \sigma}(30),{ }^{1} \tau_{2 / R \sigma}(21),{ }^{1} \tau_{3 / R \sigma}(16),{ }^{2} \tau_{1 / R 6}(7), \omega_{\mathrm{C} 9 \mathrm{O} 5}(6)$ \\
\hline 20 & 456.1 & 2.8 & 0.1 & ${ }^{2} \beta_{2 / R 6}(16), \delta_{2 / \mathrm{C}_{2} \mathrm{H}_{22} \mathrm{H} 21}(15), \delta_{2 / \mathrm{C}_{20} \mathrm{H}_{31 \mathrm{H}} 2}(10),{ }^{1} \beta_{2 / R 6}(9),{ }^{3} \beta_{2 / R 6}(6)$ \\
\hline 21 & 476.9 & 1.4 & 10.1 & ${ }^{2} \beta_{3 / R \sigma}(32),{ }^{2} \beta_{2 / R \sigma}(14),{ }^{1} \beta_{3 / R \sigma}(12),{ }^{1} \beta_{2 / R \sigma}(6),{ }^{3} \beta_{3 / R \sigma}(6), v_{\mathrm{C} 10 \mathrm{C} 11}(6)$ \\
\hline 22 & 489.7 & 1.6 & 0.0 & ${ }^{3} \tau_{2 / R \sigma}(23),{ }^{1} \tau_{3 / R \sigma}(21),{ }^{1} \tau_{2 / R \sigma}(12),{ }^{2} \tau_{2 / R 6}(9),{ }^{1} \tau_{1 / R 6}(6),{ }^{3} \tau_{3 / R 6}(5)$ \\
\hline 23 & 504.7 & 4.2 & 0.9 & $\delta_{2 / \mathrm{C}_{2} \mathrm{H} 22 \mathrm{H} 21}(23),{ }^{1} \beta_{2 / R 6}(10), \rho_{\mathrm{C}_{2} 2 \mathrm{H} 31 \mathrm{H} 32}(10),{ }^{2} \beta_{2 / R 6}(9)$ \\
\hline 24 & 557.8 & 5.2 & 0.1 & $\omega_{\mathrm{C} 18 \mathrm{~N} 3}(28),{ }^{1} \tau_{3 / R 6}(13),{ }^{1} \tau_{2 / R 6}(12),{ }^{1} \tau_{1 / R 6}(10),{ }^{2} b f l_{R R}(6)$ \\
\hline 25 & 601.9 & 5.4 & 0.4 & $\begin{array}{l}{ }^{1} \beta_{3 / R 6}(13),{ }^{3} \beta_{2 / R 6}(13), \beta_{\mathrm{C} 18 \mathrm{~N} 3 \mathrm{C} 2}(11),{ }^{1} \beta_{2 / R 6}(10), \rho_{\mathrm{C} 18 \mathrm{~N} 3}(7), \\
{ }^{2} \beta_{\text {trig/R6 }}(6),{ }^{3} \beta_{3 / R 6}(5)\end{array}$ \\
\hline 26 & 631.4 & 2.2 & 0.3 & ${ }^{2} \beta_{\text {trig/R } 6}(38),{ }^{1} \beta_{2 / R \sigma}(15), v_{\mathrm{S} 1 \mathrm{C} 20}(8), \rho_{\mathrm{C} 18 \mathrm{~N} 3}(6)$ \\
\hline 27 & 652.2 & 1.9 & 0.9 & $v_{\mathrm{S} 1 \mathrm{C} 20}(58),{ }^{3} \beta_{3 / R 6}(6)$ \\
\hline 28 & 669.0 & 3.4 & 0.2 & ${ }^{3} \tau_{1 / R 6}(36), \omega_{\mathrm{C} 9 \mathrm{O} 5}(13),{ }^{1} \tau_{1 / R 6}(11), \omega_{\mathrm{C} 18 \mathrm{~N} 3}(11)$ \\
\hline 29 & 674.2 & 13.7 & 1.7 & ${ }^{3} \beta_{3 / R \sigma}(30),{ }^{3} \beta_{2 / R \sigma}(7),{ }^{1} \tau_{1 / R \sigma}(7),{ }^{3} \tau_{1 / R \sigma}(7), v_{\mathrm{C} 10 \mathrm{C} 11}(5)$ \\
\hline 30 & 692.0 & 53.9 & 0.5 & $\tau_{\mathrm{N} 3 \mathrm{C} 18}(23), \omega_{\mathrm{N} 3 \mathrm{H} 23}(15), \tau_{\mathrm{C} 2 \mathrm{~N} 3}(9),{ }^{1} \tau_{1 / R 6}(8),{ }^{3} \beta_{3 / R 6}(7),{ }^{1} \beta_{3 / R 6}(6)$ \\
\hline 31 & 707.5 & 8.4 & 0.8 & ${ }^{3} \beta_{2 / R 6}(19), \rho_{\mathrm{C} 9 \mathrm{O} 5}(17), \rho_{\mathrm{C} 17 \mathrm{O} 4}(16),{ }^{1} \beta_{2 / R 6}(10)$ \\
\hline 32 & 724.7 & 31.5 & 0.0 & ${ }^{3} \tau_{1 / R 6}(28),{ }^{1} \tau_{1 / R 6}(26), \omega_{\mathrm{C} 6 \mathrm{H} 24}(8), \omega_{\mathrm{C} 15 \mathrm{H} 29}(7), \omega_{\mathrm{C} 17 \mathrm{O} 4}(7)$ \\
\hline 33 & 747.1 & 66.4 & 0.1 & $\begin{array}{l}{ }^{1} \tau_{1 / R 6}(15), \omega_{\mathrm{C} 18 \mathrm{~N} 3}(14), \omega_{\mathrm{C} 13 \mathrm{H} 27}(13), \omega_{\mathrm{C} 9 \mathrm{O} 5}(8), \omega_{\mathrm{C} 17 \mathrm{O} 4}(8), \\
{ }^{3} \tau_{1 / R 6}(7), \omega_{\mathrm{C} 14 \mathrm{H} 28}(7), \omega_{\mathrm{C}_{15 \mathrm{H} 29}}(6)\end{array}$ \\
\hline 34 & 780.7 & 8.4 & 1.6 & $\rho_{\mathrm{C} 20 \mathrm{H} 31 \mathrm{H} 32}(25), \beta_{\mathrm{C} 20 \mathrm{~S} 1 \mathrm{H} 33}(14),{ }^{1} \beta_{3 / R 6}(8),{ }^{1} \beta_{\text {trig/R6}}(5)$ \\
\hline 35 & 785.9 & 5.5 & 0.3 & $\begin{array}{l}{ }^{3} \tau_{1 / R 6}(18),{ }^{2} \tau_{1 / R 6}(13), \omega_{\mathrm{C} 7 \mathrm{H} 25}(12), \omega_{\mathrm{C} 9 \mathrm{O} 5}(10), \omega_{\mathrm{C} 19 \mathrm{H} 30}(10), \\
\omega_{\mathrm{C}_{17 \mathrm{O}} 4}(9), \omega_{\mathrm{C} 6 \mathrm{H} 24}(9)\end{array}$ \\
\hline 36 & 796.0 & 0.1 & 0.7 & $\begin{array}{l}{ }^{1} \beta_{\text {trig/R6 }}(13), \rho_{\mathrm{C} 20 \mathrm{H} 31 \mathrm{H} 32}(12), \beta_{\mathrm{C} 20 \mathrm{~S} 1 \mathrm{H} 33}(12),{ }^{3} \beta_{2 / R 6}(10),{ }^{1} \beta_{3 / R 6}(9), \\
{ }^{3} \beta_{\text {trig/R6 }}(7)\end{array}$ \\
\hline 37 & 808.4 & 0.5 & 0.1 & $\omega_{\mathrm{C} 9 \mathrm{O} 5}(25), \omega_{\mathrm{C} 13 \mathrm{H} 27}(13),{ }^{1} \tau_{1 / R 6}(13), \omega_{\mathrm{C} 18 \mathrm{~N} 3}(9),{ }^{2} \tau_{1 / R 6}(8)$, \\
\hline
\end{tabular}




\begin{tabular}{|c|c|c|c|c|}
\hline & & & & $\omega_{\mathrm{C} 15 \mathrm{H} 29}(7)$ \\
\hline 38 & 825.1 & 26.3 & 0.0 & $\begin{array}{l}\omega_{\mathrm{C} 7 \mathrm{H} 25}(23), \omega_{\mathrm{C} 17 \mathrm{O} 4}(18),{ }^{1} \tau_{1 / R 6}(15), \omega_{\mathrm{C} 18 \mathrm{~N} 3}(8), \omega_{\mathrm{C} 15 \mathrm{H} 29}(7), \\
{ }^{2} \tau_{1 / R 6}(7), \omega_{\mathrm{C} 6 \mathrm{H} 24}(6)\end{array}$ \\
\hline 39 & 859.2 & 13.1 & 2.4 & 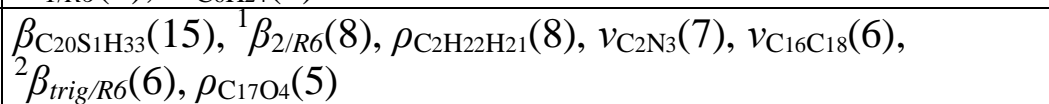 \\
\hline 40 & 910.5 & 0.9 & 0.0 & $\omega_{\mathrm{C} 19 \mathrm{H} 30}(33), \omega_{\mathrm{C} 7 \mathrm{H} 25}(23), \omega_{\mathrm{C} 12 \mathrm{H} 26}(11), \omega_{\mathrm{C} 14 \mathrm{H} 28}(10), \omega_{\mathrm{C} 15 \mathrm{H} 29}(6)$ \\
\hline 41 & 915.6 & 18.2 & 0.5 & $\begin{array}{l}\beta_{\mathrm{C} 20 \mathrm{~S} 1 \mathrm{H} 33}(21), v_{\mathrm{C} 2 \mathrm{C} 20}(17), \rho_{\mathrm{C}_{2} \mathrm{H} 22 \mathrm{H} 21}(7), \tau_{\mathrm{C} 20 \mathrm{H} 31 \mathrm{H} 32}(6), \rho_{\mathrm{C} 20 \mathrm{H} 31 \mathrm{H} 32}(5), \\
\rho_{\mathrm{C} 17 \mathrm{O} 4}(5)\end{array}$ \\
\hline 42 & 917.3 & 0.4 & 0.0 & $\begin{array}{l}\omega_{\mathrm{C} 14 \mathrm{H} 28}(20), \omega_{\mathrm{C} 12 \mathrm{H} 26}(18), \omega_{\mathrm{C} 19 \mathrm{H} 30}(17), \omega_{\mathrm{C} 7 \mathrm{H} 25}(11), \omega_{\mathrm{C} 13 \mathrm{H} 27}(10), \\
\omega_{\mathrm{C} 15 \mathrm{H} 29}(8)\end{array}$ \\
\hline 43 & 928.4 & 1.7 & 1.1 & ${ }^{1} \beta_{\text {trig/R6}}(25),{ }^{3} \beta_{\text {trig/R6 }}(24), \rho_{\mathrm{C} 9 \mathrm{O} 5}(14), v_{\mathrm{C} 16 \mathrm{C} 18}(6), \rho_{\mathrm{C} 17 \mathrm{O} 4}(6)$ \\
\hline 44 & 949.4 & 5.9 & 0.6 & $v_{\mathrm{C}_{2} \mathrm{C} 20}(36), \rho_{\mathrm{C}_{2} \mathrm{H} 22 \mathrm{H} 21}(27), v_{\mathrm{S}_{1} \mathrm{C} 20}(9), \delta_{2 / \mathrm{C} 2 \mathrm{H} 22 \mathrm{H} 21}(7)$ \\
\hline 45 & 998.4 & 0.4 & 0.0 & $\omega_{\mathrm{C} 6 \mathrm{H} 24}(51), \omega_{\mathrm{C} 19 \mathrm{H} 30}(23), \omega_{\mathrm{C} 7 \mathrm{H} 25}(10),{ }^{1} \tau_{1 / R 6}(7)$ \\
\hline 46 & 1002.7 & 2.2 & 0.0 & $\omega_{\mathrm{C} 12 \mathrm{H} 26}(35), \omega_{\mathrm{C} 14 \mathrm{H} 28}(30), \omega_{\mathrm{C} 13 \mathrm{H} 27}(12), \omega_{\mathrm{C} 15 \mathrm{H} 29}(9),{ }^{3} \tau_{3 / R 6}(5)$ \\
\hline 47 & 1010.5 & 91.8 & 1.6 & $\begin{array}{l}v_{\mathrm{C} 8 \mathrm{C} 17}(14), v_{\mathrm{C} 2 \mathrm{~N} 3}(8), v_{\mathrm{C} 11 \mathrm{C} 12}(8), \rho_{\mathrm{C} 17 \mathrm{O} 4}(6), v_{\mathrm{C} 8 \mathrm{C} 19}(6), v_{\mathrm{C} 13 \mathrm{C} 15}(6), \\
\rho_{\mathrm{C} 9 \mathrm{O} 5}(5)\end{array}$ \\
\hline 48 & 1016.1 & 0.0 & 0.0 & $\omega_{\mathrm{C} 15 \mathrm{H} 29}(31), \omega_{\mathrm{C} 13 \mathrm{H} 27}(27), \omega_{\mathrm{C} 14 \mathrm{H} 28}(17), \omega_{\mathrm{C} 12 \mathrm{H} 26}(12),{ }^{3} \tau_{1 / R 6}(12)$ \\
\hline 49 & 1057.3 & 0.5 & .4 & $v_{\mathrm{C} 13 \mathrm{C} 15}(33), v_{\mathrm{C} 14 \mathrm{C} 15}(13), \rho_{\mathrm{C} 12 \mathrm{H} 26}(11), \rho_{\mathrm{C} 14 \mathrm{H} 28}(11), v_{\mathrm{C} 12 \mathrm{C} 13}(11)$ \\
\hline 50 & 1080.1 & 8.7 & 0.2 & $\rho_{\mathrm{C} 2 \mathrm{H} 22 \mathrm{H} 21}(23), \beta_{\mathrm{C} 20 \mathrm{~S} 1 \mathrm{H} 33}(17), \rho_{\mathrm{C} 20 \mathrm{H} 31 \mathrm{H} 32}(15), v_{\mathrm{C} 2 \mathrm{C} 20}(7), v_{\mathrm{C} 7 \mathrm{C} 18}(6)$ \\
\hline 51 & 1101.5 & 23.4 & 1.5 & $v_{\mathrm{C} 6 \mathrm{C} 19}(26),{ }^{1} \beta_{\text {trig/R6}}(15), \rho_{\mathrm{C} 19 \mathrm{H} 30}(14), v_{\mathrm{C} 2 \mathrm{~N} 3}(9)$ \\
\hline 52 & 1113.1 & 1.2 & 0.3 & $\begin{array}{l}{ }^{3} \beta_{\text {trig/R6 }}(38), v_{\mathrm{C} 12 \mathrm{C} 13}(8), v_{\mathrm{C} 14 \mathrm{C} 15}(8), \rho_{\mathrm{C} 13 \mathrm{H} 27}(8), \rho_{\mathrm{C} 15 \mathrm{H} 29}(7), \\
v_{\mathrm{C} 9 \mathrm{C} 10}(6), v_{\mathrm{C} 11 \mathrm{C} 17}(5), \rho_{\mathrm{C} 14 \mathrm{H} 28}(5)\end{array}$ \\
\hline 53 & 1144.0 & 5.2 & 4.6 & $v_{\mathrm{C}_{2 \mathrm{~N}} 3}(39), v_{\mathrm{C}_{2} \mathrm{C} 20}(11), \rho_{\mathrm{C} 18 \mathrm{~N} 3}(6), v_{\mathrm{C} 8 \mathrm{C} 17}(5)$ \\
\hline 54 & 1175.6 & 5.9 & 4.5 & $\begin{array}{l}\rho_{\mathrm{C} 13 \mathrm{H} 27}(16), \rho_{\mathrm{C} 12 \mathrm{H} 26}(12), v_{\mathrm{C} 9 \mathrm{C} 16}(10), v_{\mathrm{C} 9 \mathrm{C} 10}(7),{ }^{1} \beta_{\text {trig/R6 }}(7), \\
{ }^{3} \beta_{\text {trig/R6}}(6)\end{array}$ \\
\hline 55 & .9 & & 0.6 & $\rho_{\mathrm{C} 15 \mathrm{H} 29}(33), \rho_{\mathrm{C} 13 \mathrm{H} 27}(10), \rho_{\mathrm{C} 14 \mathrm{H} 28}(10), v_{\mathrm{C} 14 \mathrm{C} 15}(9), v_{\mathrm{C} 13 \mathrm{C} 15}(6)$ \\
\hline 56 & 1199.5 & 28.8 & 8.2 & $\rho_{\mathrm{C} 6 \mathrm{H} 24}(31), \rho_{\mathrm{C} 7 \mathrm{H} 25}(13), v_{\mathrm{C} 6 \mathrm{C} 7}(8), \rho_{\mathrm{C} 13 \mathrm{H} 27}(5)$ \\
\hline 57 & 1215.9 & 1.8 & .0 & $\rho_{\mathrm{C} 19 \mathrm{H} 30}(26), \rho_{\mathrm{C} 7 \mathrm{H} 25}(10), v_{\mathrm{C} 8 \mathrm{C} 19}(9), v_{\mathrm{C}_{11} \mathrm{C} 17}(8), \mu$ \\
\hline 58 & 1222.0 & 10.1 & 1.0 & $\tau_{\mathrm{C} 20 \mathrm{H} 31 \mathrm{H} 32}(66), \tau_{\mathrm{C}_{2} \mathrm{H} 22 \mathrm{H} 21}(7), \omega_{\mathrm{C} 2 \mathrm{H} 22 \mathrm{H} 21}(6)$ \\
\hline 59 & 1250.4 & 107.3 & 1.8 & $\begin{array}{l}\omega_{\mathrm{C} 20 \mathrm{H} 31 \mathrm{H} 32}(14), v_{\mathrm{C} 7 \mathrm{C} 18}(10), \tau_{\mathrm{C} 2 \mathrm{H} 22 \mathrm{H} 21}(10), \rho_{\mathrm{C} 12 \mathrm{H} 26}(9), v_{\mathrm{C} 9 \mathrm{C} 10}(7), \\
\tau_{\mathrm{C} 20 \mathrm{H} 31 \mathrm{H} 32}(6)\end{array}$ \\
\hline 60 & 1275.2 & 51.6 & 0.3 & $\begin{array}{l}\omega_{\mathrm{C} 20 \mathrm{H} 31 \mathrm{H} 32}(17), \tau_{\mathrm{C} 2 \mathrm{H} 22 \mathrm{H} 21}(14), \rho_{\mathrm{C} 14 \mathrm{H} 28}(11),{ }^{2} \beta_{\text {trig/R6 }}(11), \\
\rho_{\mathrm{C} 12 \mathrm{H} 26}(10)\end{array}$ \\
\hline 61 & 1293.3 & 552.5 & 10.7 & $v_{\mathrm{C} 9 \mathrm{C} 10}(12), \omega_{\mathrm{C} 20 \mathrm{H} 31 \mathrm{H} 32}(9), v_{\mathrm{C} 9 \mathrm{C} 16}(9), v_{\mathrm{C}_{11} \mathrm{C} 17}(6), v_{\mathrm{C} 8 \mathrm{C} 16}(6), v_{\mathrm{C} 8 \mathrm{C} 17}(5)$ \\
\hline 62 & 1318.1 & 113.9 & 1.8 & $\begin{array}{l}v_{\mathrm{C} 11 \mathrm{C} 17}(18), v_{\mathrm{C} 8 \mathrm{C} 17}(14), \rho_{\mathrm{C} 12 \mathrm{H} 26}(8), \rho_{\mathrm{C} 17 \mathrm{O} 4}(7),{ }^{2} \beta_{2 / R 6}(6), \\
\omega_{\mathrm{C} 20 \mathrm{H} 31 \mathrm{H} 32}(6)\end{array}$ \\
\hline 63 & 1334.0 & 103.4 & 1.8 & $\begin{array}{l}\tau_{\mathrm{C}_{2 \mathrm{H} 22 \mathrm{H} 21}}(18), \omega_{\mathrm{C}_{20} \mathrm{H} 31 \mathrm{H} 32}(16), v_{\mathrm{N}_{3} \mathrm{C} 18}(9), \rho_{\mathrm{N}_{3} \mathrm{H} 23}(9), \omega_{\mathrm{C}_{2} \mathrm{H} 22 \mathrm{H} 21}(8), \\
v_{\mathrm{C} 16 \mathrm{C} 18}(6), v_{\mathrm{C} 9 \mathrm{C} 16}(6)\end{array}$ \\
\hline 64 & 1340.1 & 84.0 & 4.8 & $\begin{array}{l}\omega_{\mathrm{C} 20 \mathrm{H} 31 \mathrm{H} 32}(22), v_{\mathrm{N} 3 \mathrm{C} 18}(18), \tau_{\mathrm{C}_{2 \mathrm{H} 22 \mathrm{H} 21}}(16), v_{\mathrm{C} 16 \mathrm{C} 18}(6), \rho_{\mathrm{N} 3 \mathrm{H} 23}(6), \\
{ }^{1} \beta_{\text {trig/R6 }}(6)\end{array}$ \\
\hline 65 & 1358.5 & 13.9 & 0.9 & $\begin{array}{l}v_{\mathrm{C} 10 \mathrm{C} 11}(15), v_{\mathrm{C} 10 \mathrm{C} 14}(15), v_{\mathrm{C} 11 \mathrm{C} 12}(14), v_{\mathrm{C} 13 \mathrm{C} 15}(14), v_{\mathrm{C} 12 \mathrm{C} 13}(13), \\
v_{\mathrm{C} 14 \mathrm{C} 15}(13)\end{array}$ \\
\hline 66 & 1377.8 & 20.9 & 1.8 & $\begin{array}{l}v_{\mathrm{C} 16 \mathrm{C} 18}(18), v_{\mathrm{C} 8 \mathrm{C} 19}(11), v_{\mathrm{C} 8 \mathrm{C} 16}(10), v_{\mathrm{C} 9 \mathrm{C} 16}(9), v_{\mathrm{C} 6 \mathrm{C} 19}(7), v_{\mathrm{C} 6 \mathrm{C} 7}(6), \\
v_{\mathrm{C} 7 \mathrm{C} 18}(6), \rho_{\mathrm{C} 9 \mathrm{O} 5}(6), v_{\mathrm{C} 9 \mathrm{C} 10}(5)\end{array}$ \\
\hline 67 & 1395.6 & 42.5 & 2.3 & $\omega_{\mathrm{C} 2 \mathrm{H} 22 \mathrm{H} 21}(67)$ \\
\hline 68 & 1436.1 & 34.2 & 0.5 & $\rho_{\mathrm{C} 7 \mathrm{H} 25}(24), v_{\mathrm{C} 6 \mathrm{C} 7}(11), v_{\mathrm{C} 8 \mathrm{C} 19}(11), v_{\mathrm{C} 8 \mathrm{C} 16}(7), v_{\mathrm{C} 9 \mathrm{C} 16}(6)$ \\
\hline 69 & 1462.8 & 6.2 & 1.1 & $\delta_{1 / \mathrm{C} 20 \mathrm{H} 31 \mathrm{H} 32}(93)$ \\
\hline 70 & 1478.7 & 0.7 & 3.3 & $\rho_{\mathrm{C} 15 \mathrm{H} 29}(16), \rho_{\mathrm{C} 13 \mathrm{H} 27}(14), v_{\mathrm{C} 10 \mathrm{C} 14}(7), v_{\mathrm{C} 11 \mathrm{C} 12}(7), \rho_{\mathrm{C} 19 \mathrm{H} 30}(6)$ \\
\hline 71 & 1487.8 & 7.8 & 0.9 & $\delta_{1 / \mathrm{C} 2 \mathrm{H} 22 \mathrm{H} 21}(12), \rho_{\mathrm{C} 19 \mathrm{H} 30}(10), v_{\mathrm{C} 8 \mathrm{C} 16}(7), \rho_{\mathrm{C} 6 \mathrm{H} 24}(7), \rho_{\mathrm{C} 13 \mathrm{H} 27}(6)$, \\
\hline
\end{tabular}




\begin{tabular}{|c|c|c|c|c|}
\hline & & & & $\rho_{\mathrm{C} 15 \mathrm{H} 29}(5), v_{\mathrm{C} 8 \mathrm{C} 19}(5)$ \\
\hline 72 & 1503.7 & 5.2 & 1.7 & $\delta_{1 / \mathrm{C}_{2} \mathrm{H} 22 \mathrm{H} 21}(67)$ \\
\hline 73 & 1508.4 & 3.7 & 1.5 & $\rho_{\mathrm{C} 14 \mathrm{H} 28}(18), \rho_{\mathrm{C} 12 \mathrm{H} 26}(17), v_{\mathrm{C} 10 \mathrm{C} 11}(15), v_{\mathrm{C}_{13} \mathrm{C} 15}(7), \delta_{1 / \mathrm{C}_{2} \mathrm{H} 22 \mathrm{H} 21}(6)$ \\
\hline 74 & 1555.0 & 188.9 & 1.4 & $v_{\mathrm{C} 6 \mathrm{C} 19}(19), \rho_{\mathrm{N} 3 \mathrm{H} 23}(18), v_{\mathrm{N} 3 \mathrm{C} 18}(15), \rho_{\mathrm{C} 6 \mathrm{H} 24}(12), v_{\mathrm{C} 6 \mathrm{C} 7}(8), v_{\mathrm{C} 8 \mathrm{C} 16}(6)$ \\
\hline 75 & 1611.9 & 42.9 & 10.6 & $\begin{array}{l}v_{\mathrm{C} 13 \mathrm{C} 15}(12), v_{\mathrm{C} 10 \mathrm{C} 11}(12), v_{\mathrm{O} 5 \mathrm{C} 9}(8), v_{\mathrm{C} 8 \mathrm{C} 19}(6), v_{\mathrm{C} 12 \mathrm{C} 13}(6), \rho_{\mathrm{N} 3 \mathrm{H} 23}(6) \\
v_{\mathrm{C} 6 \mathrm{C} 19}(6), v_{\mathrm{C} 16 \mathrm{C} 18}(5)\end{array}$ \\
\hline 76 & 1614.7 & 76.9 & 5.8 & $\begin{array}{l}\rho_{\mathrm{N}_{3} \mathrm{H} 23}(18), v_{\mathrm{C} 10 \mathrm{C} 11}(8), v_{\mathrm{C} 13 \mathrm{C} 15}(8), v_{\mathrm{C} 6 \mathrm{C} 19}(7), v_{\mathrm{C} 8 \mathrm{C} 19}(7), v_{\mathrm{C} 7 \mathrm{C} 18}(6) \\
v_{\mathrm{C} 16 \mathrm{C} 18}(6)\end{array}$ \\
\hline 77 & 1627.5 & 32.9 & 15.2 & $v_{\mathrm{C}_{14} \mathrm{C} 15}(15), v_{\mathrm{C} 11 \mathrm{C} 12}(11), v_{\mathrm{C}_{12} \mathrm{C} 13}(8), v_{\mathrm{O} 5 \mathrm{C} 9}(8),{ }^{3} \beta_{2 / R 6}(7)$ \\
\hline 78 & 1631.2 & 290.1 & 4.7 & $\begin{array}{l}v_{\mathrm{C} 6 \mathrm{C} 7}(17), v_{\mathrm{C} 8 \mathrm{C} 19}(7),{ }_{3 / R 6}^{1}(6), \rho_{\mathrm{C} 7 \mathrm{H} 25}(6), v_{\mathrm{N} 3 \mathrm{C} 18}(6), v_{\mathrm{O} 4 \mathrm{C} 17}(6), \\
v_{\mathrm{C}_{14} \mathrm{C} 15}(5), v_{\mathrm{C} 8 \mathrm{C} 16}(5)\end{array}$ \\
\hline 79 & 1672.1 & 201.8 & 28.7 & $v_{\mathrm{O} 5 \mathrm{C} 9}(46),{ }^{2} \beta_{3 / R 6}(6),{ }^{2} \beta_{\text {trig/R6 }}(6)$ \\
\hline 80 & 1720.6 & 182.0 & 28.2 & $v_{\mathrm{O} 4 \mathrm{C} 17}(71),{ }^{2} \beta_{\text {trig/R6 }}(9)$ \\
\hline 81 & 2657.1 & 6.1 & 10.8 & $v_{\mathrm{S}_{1 H} 33}(100)$ \\
\hline 82 & 3021.3 & 45.7 & 7.5 & $v_{\mathrm{C} 2 \mathrm{H} 21}(86), v_{\mathrm{C} 2 \mathrm{H} 22}(11)$ \\
\hline 83 & 3057.2 & 24.0 & 12.7 & $v_{\mathrm{C} 20 \mathrm{H} 32}(56), v_{\mathrm{C} 20 \mathrm{H} 31}(36), v_{\mathrm{C}_{2} \mathrm{H} 22}(8)$ \\
\hline 84 & 3075.0 & 15.4 & 4.9 & $v_{\mathrm{C}_{2} \mathrm{H} 22}(75), v_{\mathrm{C}_{2} \mathrm{H} 21}(13), v_{\mathrm{C}_{20} \mathrm{H} 32}(11)$ \\
\hline 85 & 3110.9 & 19.7 & 3.4 & $v_{\mathrm{C} 20 \mathrm{H} 31}(63), v_{\mathrm{C} 20 \mathrm{H} 32}(31)$ \\
\hline 86 & 3169.7 & 3.5 & 4.5 & $v_{\mathrm{C} 15 \mathrm{H} 29}(50), v_{\mathrm{C} 13 \mathrm{H} 27}(44)$ \\
\hline 87 & 3171.5 & 11.7 & 7.9 & $v_{\mathrm{C} 6 \mathrm{H} 24}(90), v_{\mathrm{C} 7 \mathrm{H} 25}(7)$ \\
\hline 88 & 3184.5 & 13.7 & 14.7 & $v_{\mathrm{C} 13 \mathrm{H} 27}(43), v_{\mathrm{C} 15 \mathrm{H} 29}(42), v_{\mathrm{C} 12 \mathrm{H} 26}(9)$ \\
\hline 89 & 3201.3 & 4.1 & 7.2 & $v_{\mathrm{C} 12 \mathrm{H} 26}(82), v_{\mathrm{C} 13 \mathrm{H} 27}(9), v_{\mathrm{C} 14 \mathrm{H} 28}(8)$ \\
\hline 90 & 3204.5 & 12.8 & 8.1 & $v_{\mathrm{C} 7 \mathrm{H} 25}(90)$ \\
\hline 91 & 3205.8 & 8.4 & 12.9 & $v_{\mathrm{C} 14 \mathrm{H} 28}(84), v_{\mathrm{C} 15 \mathrm{H} 29}(8), v_{\mathrm{C} 12 \mathrm{H} 26}(6)$ \\
\hline 92 & 3213.0 & 4.2 & 12.7 & $v_{\mathrm{C} 19 \mathrm{H} 30}(92)$ \\
\hline 93 & 3470.1 & 97.5 & 30.0 & $v_{\mathrm{N} 3 \mathrm{H} 23}(99)$ \\
\hline
\end{tabular}


Table S.12. Frequencies of normal vibrations, IR and Raman intensities and PEDs for $S c G-G$ conformer calculated at the B3LYP/6-311++G(d,p) level of theory.

\begin{tabular}{|c|c|c|c|c|}
\hline $\mathbf{N r}$ & $\begin{array}{l}\text { Freq. } \\
{\left[\mathrm{cm}^{-1}\right]}\end{array}$ & IR Int. & $\begin{array}{c}\text { Raman } \\
\text { Int. }\end{array}$ & PED $($ contributions $>5 \%)$ \\
\hline 1 & 25.3 & 0.6 & 10.6 & $\tau_{\mathrm{N} 3 \mathrm{C} 18}(46), \omega_{\mathrm{N} 3 \mathrm{H} 23}(38)$ \\
\hline 2 & 31.5 & 2.6 & 7.5 & ${ }^{2} \tau_{3 / R 6}(31), \tau_{\mathrm{C} 2 \mathrm{~N} 3}(26),{ }^{1} \tau_{3 / R \sigma}(19)$ \\
\hline 3 & 50.8 & 2.3 & 1.9 & ${ }^{2} \tau_{3 / R \sigma}(29), \tau_{\mathrm{C}_{2} \mathrm{~N} 3}(23),{ }^{2} \tau_{2 / R \sigma}(20), \omega_{\mathrm{N} 3 \mathrm{H} 23}(11)$ \\
\hline 4 & 67.7 & 0.3 & 0.6 & $\begin{array}{l}\tau_{\mathrm{C} 2 \mathrm{C} 20}(20),{ }^{2} \tau_{2 / R 6}(19),{ }^{1} \tau_{3 / R 6}(15),{ }^{2} \tau_{3 / R 6}(7),{ }^{1} \tau_{2 / R 6}(6), \omega_{\mathrm{N} 3 \mathrm{H} 23}(6), \\
\tau_{\mathrm{N} 3 \mathrm{C} 18}(6)\end{array}$ \\
\hline 5 & 106.8 & 0.7 & 0.2 & $\tau_{\mathrm{C}_{2} \mathrm{C} 20}(27),{ }^{2} \tau_{2 / R 6}(17), \omega_{\mathrm{N} 3 \mathrm{H} 23}(14), \tau_{\mathrm{N} 3 \mathrm{C} 18}(8), \beta_{\mathrm{C}_{18 \mathrm{~N} 3 \mathrm{C} 2}(7),{ }^{3} \tau_{2 / R 6}(6)}$ \\
\hline 6 & 120.8 & 0.4 & 0.9 & ${ }^{2} \tau_{1 / R 6}(42),{ }^{3} \tau_{2 / R 6}(14), \omega_{\mathrm{N} 3 \mathrm{H} 23}(8),{ }^{3} \tau_{3 / R 6}(7),{ }^{2} \tau_{2 / R 6}(5)$ \\
\hline 7 & 162.0 & 1.0 & 0.4 & $\begin{array}{l}{ }^{4} b f l_{R R}(11),{ }^{1} \tau_{2 / R 6}(11),{ }^{2} b f l_{R R}(9), \delta_{2 / C 20 H 31 H 32}(7),{ }^{l} \tau_{3 / R 6}(7), \\
\tau_{N 3 C l 8}(6),{ }^{2} \tau_{3 / R 6}(6), \rho_{C 18 N 3}(6), \omega_{C 19 H 30}(5), \beta_{C l 8 N_{3} C_{2}}(5)\end{array}$ \\
\hline 8 & 170.6 & 0.2 & 0.6 & ${ }^{4} b f l_{R R}(20),{ }^{2} b f l_{R R}(15), \delta_{2 / C 20 H 3 l H 32}(8),{ }^{2} \tau_{2 / R \sigma}(6), \tau_{N 3 C l 8}(5)$ \\
\hline 9 & 199.3 & 5.6 & 0.8 & ${ }^{1}{ }^{1} \tau_{2 / R 6}(15),{ }^{1} \tau_{3 / R \sigma}(14), \tau_{\mathrm{C}_{2 N} \mathrm{~N}}(8), \tau_{\mathrm{S} 1 \mathrm{C} 20}(7), \tau_{\mathrm{C} 2 \mathrm{C} 20}(5)$ \\
\hline 10 & 213.3 & 4.3 & 0.4 & 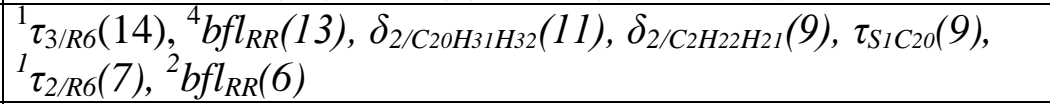 \\
\hline 11 & 237.5 & 19.2 & 1.0 & $\tau_{\mathrm{S} 1 \mathrm{C} 20}(71)$ \\
\hline 12 & 267.0 & 0.5 & 0.6 & $\begin{array}{l}{ }^{2} b f l_{R R}(21), \delta_{2 / C 20 H 31 H 32}(8), \omega_{C 18 N_{3}}(7),{ }^{l} \tau_{2 / R 6}(6),{ }^{l} \tau_{3 / R 6}(6), \\
\omega_{N_{3} H_{23}(6)}\end{array}$ \\
\hline 13 & 281.9 & 2.6 & 0.4 & ${ }^{2} \beta_{2 / R 6}(22), \rho_{\mathrm{C} 18 \mathrm{~N} 3}(13), v_{\mathrm{C} 8 \mathrm{C} 17}(7),{ }^{2} b f l_{R R}(6)$ \\
\hline 14 & 344.0 & 1.4 & 0.1 & ${ }^{2} \beta_{3 / R 6}(29), \rho_{\mathrm{C} 17 \mathrm{O} 4}(20), \rho_{\mathrm{C} 18 \mathrm{~N} 3}(9), v_{\mathrm{C} 8 \mathrm{C} 17}(8)$ \\
\hline 15 & 363.3 & 3.9 & 0.3 & $\begin{array}{l}{ }^{2} \beta_{2 / R 6}(21), \rho_{\mathrm{C} 18 \mathrm{~N} 3}(11), \delta_{2 / \mathrm{C}_{20} \mathrm{H}_{31} \mathrm{H}_{32}}(10), \beta_{\mathrm{C}_{18 \mathrm{~N} 3 \mathrm{C} 2}}(9), \tau_{\mathrm{C}_{2} \mathrm{C} 20}(8), \\
v_{\mathrm{C} 9 \mathrm{C} 10}(6)\end{array}$ \\
\hline 16 & 376.2 & 6.3 & 0.8 & ${ }^{2} \beta_{3 / R 6}(18),{ }^{1} \beta_{3 / R 6}(13), \delta_{2 / \mathrm{C} 20 \mathrm{H} 31 \mathrm{H} 32}(10), v_{\mathrm{C} 11 \mathrm{C} 17}(9)$ \\
\hline 17 & 426.0 & 7.1 & 0.2 & ${ }^{3} \tau_{3 / R 6}(46),{ }^{4} b f l_{R R}(11), \rho_{C 9 O 5}(8),{ }^{3} \tau_{2 / R 6}(6)$ \\
\hline 18 & 427.3 & 13.6 & 0.4 & ${ }^{3} \tau_{3 / R 6}(37), \rho_{\mathrm{C} 9 \mathrm{O} 5}(16),{ }^{4} b f l_{R R}(9), \rho_{\mathrm{Cl}_{17 O 4}}(7)$ \\
\hline 19 & 432.5 & 0.0 & 0.1 & ${ }^{3} \tau_{2 / R 6}(31),{ }^{1} \tau_{2 / R 6}(21),{ }^{1} \tau_{3 / R 6}(17),{ }^{2} \tau_{1 / R 6}(6), \omega_{\mathrm{C} 9 \mathrm{O} 5}(6)$ \\
\hline 20 & 453.0 & 2.8 & 0.1 & $\delta_{2 / \mathrm{C}_{2} \mathrm{H} 22 \mathrm{H} 21}(17),{ }^{2} \beta_{2 / R 6}(17), \delta_{2 / \mathrm{C}_{20} \mathrm{H} 31 \mathrm{H} 32}(10),{ }_{2 / R 6}(9),{ }^{3} \beta_{2 / R 6}(5)$ \\
\hline 21 & 477.0 & 1.4 & 10.1 & ${ }^{2} \beta_{3 / R 6}(32),{ }^{2} \beta_{2 / R 6}(13),{ }^{1} \beta_{3 / R 6}(12),{ }^{1} \beta_{2 / R 6}(7),{ }^{3} \beta_{3 / R 6}(6), v_{\mathrm{C} 10 \mathrm{C} 11}(5)$ \\
\hline 22 & 490.1 & 1.1 & 0.0 & ${ }^{3} \tau_{2 / R \sigma}(23),{ }^{1} \tau_{3 / R \sigma}(21),{ }^{1} \tau_{2 / R \sigma}(12),{ }^{2} \tau_{2 / R 6}(9),{ }^{3} \tau_{3 / R \sigma}(6),{ }^{1} \tau_{1 / R \sigma}(5)$ \\
\hline 23 & 504.1 & 3.5 & 0.9 & $\delta_{2 / \mathrm{C} 2 \mathrm{H} 22 \mathrm{H} 21}(24),{ }^{1} \beta_{2 / R 6}(10),{ }^{2} \beta_{2 / R 6}(9), \rho_{\mathrm{C}_{2} \mathrm{H} 31 \mathrm{H} 32}(8)$ \\
\hline 24 & 557.7 & 4.0 & 0.1 & $\omega_{\mathrm{C} 18 \mathrm{~N} 3}(29),{ }^{1} \tau_{3 / R \sigma}(13),{ }^{1} \tau_{2 / R \sigma}(13),{ }^{1} \tau_{1 / R 6}(10),{ }^{2} b f l_{R R}(6)$ \\
\hline 25 & 603.2 & 3.2 & 0.4 & $\begin{array}{l}{ }^{1} \beta_{3 / R 6}(13),{ }^{3} \beta_{2 / R 6}(13),{ }^{1} \beta_{2 / R 6}(11), \beta_{\mathrm{C}_{18 \mathrm{~N} 3 \mathrm{C} 2}}(11), \rho_{\mathrm{C} 18 \mathrm{~N} 3}(7), \\
{ }^{2} \beta_{\text {trig/R6}}(6),{ }^{3} \beta_{3 / R 6}(5)\end{array}$ \\
\hline 26 & 631.5 & 1.9 & 0.3 & ${ }^{2} \beta_{\text {trig/R6}}(38),{ }^{1} \beta_{2 / R 6}(14), v_{\mathrm{S} 1 \mathrm{C} 20}(8), \rho_{\mathrm{C} 18 \mathrm{~N} 3}(6)$ \\
\hline 27 & 659.5 & 3.6 & 0.9 & $v_{\mathrm{S} 1 \mathrm{C} 20}(51),{ }^{3} \beta_{3 / R 6}(11), \delta_{2 / \mathrm{C}_{20} \mathrm{H} 31 \mathrm{H} 32}(6)$ \\
\hline 28 & 670.4 & 1.0 & 0.1 & ${ }^{3} \tau_{1 / R 6}(39),{ }^{1} \tau_{1 / R 6}(16), \omega_{\mathrm{C} 9 \mathrm{O} 5}(12), \omega_{\mathrm{C} 18 \mathrm{~N} 3}(12)$ \\
\hline 29 & 678.3 & 9.1 & 2.4 & ${ }^{3} \beta_{3 / R 6}(33), v_{\mathrm{S}_{1} \mathrm{C} 20}(10), \beta_{\mathrm{C} 18 \mathrm{~N} 3 \mathrm{C} 2}(7), v_{\mathrm{C} 10 \mathrm{C} 11}(6)$ \\
\hline 30 & 703.5 & 38.5 & 0.3 & $\begin{array}{l}\tau_{\mathrm{N} 3 \mathrm{C} 18}(18), \omega_{\mathrm{N} 3 \mathrm{H} 23}(13), \tau_{\mathrm{C} 2 \mathrm{~N} 3}(9), \rho_{\mathrm{C} 9 \mathrm{O} 5}(8),{ }^{1} \tau_{1 / R 6}(8), \rho_{\mathrm{C} 17 \mathrm{O} 4}(7), \\
3_{2 / R 6}(7)\end{array}$ \\
\hline 31 & 709.7 & 31.4 & 0.5 & $\begin{array}{l}{ }^{3} \beta_{2 / R 6}(13), \rho_{\mathrm{C} 9 \mathrm{O} 5}(12), \rho_{\mathrm{C} 17 \mathrm{O} 4}(11), \tau_{\mathrm{N} 3 \mathrm{C} 18}(10), \omega_{\mathrm{N} 3 \mathrm{H} 23}(6),{ }^{1} \beta_{2 / R 6}(6), \\
\tau_{\mathrm{C} 2 \mathrm{~N} 3}(5)\end{array}$ \\
\hline 32 & 725.7 & 24.7 & 0.0 & ${ }^{3} \tau_{1 / R 6}(28),{ }^{1} \tau_{1 / R 6}(24), \omega_{\mathrm{C} 15 \mathrm{H} 29}(8), \omega_{\mathrm{C} 17 \mathrm{O} 4}(7), \omega_{\mathrm{C} 6 \mathrm{H} 24}(7)$ \\
\hline 33 & 747.5 & 74.3 & 0.0 & $\begin{array}{l}{ }_{\tau_{1 / R 6}}(14), \omega_{\mathrm{C} 18 \mathrm{~N} 3}(13), \omega_{\mathrm{C} 13 \mathrm{H} 27}(13), \omega_{\mathrm{C} 9 \mathrm{O} 5}(8),{ }^{3} \tau_{1 / R 6}(8), \\
\omega_{\mathrm{C} 17 \mathrm{O} 4}(7), \omega_{\mathrm{C} 14 \mathrm{H} 28}(6), \omega_{\mathrm{C} 15 \mathrm{H} 29}(6)\end{array}$ \\
\hline 34 & 756.1 & 6.0 & 0.9 & $\rho_{\mathrm{C} 20 \mathrm{H} 31 \mathrm{H} 32}(35), \beta_{\mathrm{C} 20 \mathrm{~S} 1 \mathrm{H} 33}(27), \rho_{\mathrm{C} 2 \mathrm{H} 22 \mathrm{H} 21}(7)$ \\
\hline 35 & 787.4 & 4.4 & 0.1 & $\begin{array}{l}{ }^{3} \tau_{1 / R 6}(18), \omega_{\mathrm{C} 7 \mathrm{H} 25}(14),{ }^{2} \tau_{1 / R 6}(13), \omega_{\mathrm{C} 6 \mathrm{H} 24}(11), \omega_{\mathrm{C} 19 \mathrm{H} 30}(11), \\
\omega_{\mathrm{C} 9 \mathrm{O} 5}(10), \omega_{\mathrm{C} 17 \mathrm{O} 4}(10)\end{array}$ \\
\hline
\end{tabular}




\begin{tabular}{|c|c|c|c|c|}
\hline 36 & 794.5 & 1.6 & 1.0 & $\begin{array}{l}{ }^{1} \beta_{\text {trig/R6}}(18),{ }^{1} \beta_{3 / R \sigma}(14),{ }^{3} \beta_{2 / R \sigma}(12),{ }^{3} \beta_{\text {trig/R6 }}(9), v_{\mathrm{C} 9 \mathrm{C} 10}(6), \\
{ }^{2} \beta_{\text {trig/R6}}(6)\end{array}$ \\
\hline 37 & 808.5 & 0.5 & 0.1 & $\begin{array}{l}\omega_{\mathrm{C} 9 \mathrm{O} 5}(25), \omega_{\mathrm{C} 13 \mathrm{H} 27}(13),{ }^{1} \tau_{1 / R 6}(12), \omega_{\mathrm{C} 18 \mathrm{~N} 3}(9),{ }^{2} \tau_{1 / R 6}(8), \\
\omega_{\mathrm{C} 15 \mathrm{H} 29}(7)\end{array}$ \\
\hline 38 & 826.0 & 24.8 & 0.0 & $\begin{array}{l}\omega_{\mathrm{C} 7 \mathrm{H} 25}(23), \omega_{\mathrm{C} 17 \mathrm{O} 4}(19),{ }^{1} \tau_{1 / R 6}(14), \omega_{\mathrm{C} 18 \mathrm{~N} 3}(7), \omega_{\mathrm{C} 15 \mathrm{H} 29}(7), \\
{ }^{2} \tau_{1 / R 6}(7), \omega_{\mathrm{C} 6 \mathrm{H} 24}(6)\end{array}$ \\
\hline 39 & 865.6 & 16.1 & 2.9 & $\begin{array}{l}{ }^{1} \beta_{2 / R 6}(8), \beta_{\mathrm{C} 20 \mathrm{~S} 1 \mathrm{H} 33}(8), v_{\mathrm{C} 16 \mathrm{C} 18}(8), v_{\mathrm{C} 2 \mathrm{~N} 3}(7), \rho_{\mathrm{C} 1704}(7), v_{\mathrm{C} 7 \mathrm{C} 18}(7), \\
{ }^{2} \beta_{\text {trig/R6}}(6), v_{\mathrm{N} 3 \mathrm{C} 18}(6), v_{\mathrm{C} 11 \mathrm{C} 17}(5)\end{array}$ \\
\hline 40 & 911.9 & 0.6 & 0.0 & $\omega_{\mathrm{C} 19 \mathrm{H} 30}(32), \omega_{\mathrm{C} 7 \mathrm{H} 25}(22), \omega_{\mathrm{C} 12 \mathrm{H} 26}(12), \omega_{\mathrm{C} 14 \mathrm{H} 28}(12), \omega_{\mathrm{C}_{15 \mathrm{H}} 29}(6)$ \\
\hline 41 & 918.6 & 0.5 & 0.0 & $\begin{array}{l}\omega_{\mathrm{C} 14 \mathrm{H} 28}(19), \omega_{\mathrm{C} 19 \mathrm{H} 30}(18), \omega_{\mathrm{C} 12 \mathrm{H} 26}(17), \omega_{\mathrm{C} 7 \mathrm{H} 25}(12), \omega_{\mathrm{C} 13 \mathrm{H} 27}(10), \\
\omega_{\mathrm{C} 15 \mathrm{H} 29}(7)\end{array}$ \\
\hline 42 & 926.6 & 11.8 & 0.4 & $\begin{array}{l}v_{\mathrm{C} 2 \mathrm{C} 20}(21), \rho_{\mathrm{C} 9 \mathrm{O} 5}(13),{ }^{3} \beta_{\text {trig/R6}}(10),{ }^{1} \beta_{\text {trig/R6 }}(9), v_{\mathrm{C} 16 \mathrm{C} 18}(9) \\
\beta_{\mathrm{C}_{20 \mathrm{~S} 1 \mathrm{H} 33}(5)}\end{array}$ \\
\hline 43 & 930.6 & 2.1 & 1.2 & ${ }^{1} \beta_{\text {trig/R6}}(18), v_{\mathrm{C} 2 \mathrm{C} 20}(18),{ }^{3} \beta_{\text {trig/R6}}(17), \rho_{\mathrm{C} 17 \mathrm{O} 4}(9)$ \\
\hline 44 & 972.9 & 14.6 & 0.1 & $\rho_{\mathrm{C}_{2} \mathrm{H} 22 \mathrm{H} 21}(37), \beta_{\mathrm{C}_{20 \mathrm{~S} 1 \mathrm{H} 33}}(11), v_{\mathrm{C}_{2} \mathrm{C} 20}(10)$ \\
\hline 45 & 999.8 & 0.1 & 0.0 & $\omega_{\mathrm{C} 6 \mathrm{H} 24}(51), \omega_{\mathrm{C} 19 \mathrm{H} 30}(23), \omega_{\mathrm{C} 7 \mathrm{H} 25}(10),{ }^{1} \tau_{1 / R 6}(7)$ \\
\hline 46 & 1003.7 & 2.1 & 0.0 & $\omega_{\mathrm{C} 12 \mathrm{H} 26}(34), \omega_{\mathrm{C} 14 \mathrm{H} 28}(29), \omega_{\mathrm{C} 13 \mathrm{H} 27}(12), \omega_{\mathrm{C} 15 \mathrm{H} 29}(9),{ }^{3} \tau_{3 / R 6}(5)$ \\
\hline 47 & 1016.6 & 78.3 & 1.9 & $v_{\mathrm{C} 8 \mathrm{C} 17}(13), \beta_{\mathrm{C}_{20} \mathrm{~S} 1 \mathrm{H} 33}(8), v_{\mathrm{C} 11 \mathrm{C} 12}(6), v_{\mathrm{C}_{13} \mathrm{C} 15}(6), \rho_{\mathrm{C} 20 \mathrm{H} 31 \mathrm{H} 32}(5)$ \\
\hline 48 & 1017.3 & 1.0 & 0.0 & $\omega_{\mathrm{C} 15 \mathrm{H} 29}(30), \omega_{\mathrm{C} 13 \mathrm{H} 27}(27), \omega_{\mathrm{C} 14 \mathrm{H} 28}(16), \omega_{\mathrm{C} 12 \mathrm{H} 26}(12),{ }^{3} \tau_{1 / R 6}(12)$ \\
\hline 49 & 1050.2 & 4.8 & 0.8 & \begin{tabular}{|l|}
$\rho_{\mathrm{C} 20 \mathrm{H} 31 \mathrm{H} 32}(24), \rho_{\mathrm{C} 2 \mathrm{H} 22 \mathrm{H} 21}(17), \beta_{\mathrm{C}_{20} \mathrm{~S} 1 \mathrm{H} 33}(15), v_{\mathrm{C}_{2} \mathrm{C} 20}(11)$ \\
$\delta_{2 / \mathrm{C} 2 \mathrm{H} 22 \mathrm{H} 21}(6)$
\end{tabular} \\
\hline 50 & 1059.2 & 0.6 & 5.0 & $v_{\mathrm{C} 13 \mathrm{C} 15}(28), \rho_{\mathrm{C} 12 \mathrm{H} 26}(11), v_{\mathrm{C} 14 \mathrm{C} 15}(11), \rho_{\mathrm{C} 14 \mathrm{H} 28}(10), v_{\mathrm{C} 12 \mathrm{C} 13}(8)$ \\
\hline 51 & 1099.0 & 27.8 & 1.6 & $v_{\mathrm{C} 6 \mathrm{C} 19}(30), \rho_{\mathrm{C} 19 \mathrm{H} 30}(16),{ }_{\text {trig/R6}}(14), v_{\mathrm{C} 6 \mathrm{C} 7}(7), v_{\mathrm{C} 2 \mathrm{~N} 3}(6), v_{\mathrm{C} 9 \mathrm{C} 16}(5)$ \\
\hline 52 & 1113.3 & 1.1 & 0.3 & $\begin{array}{l}{ }^{3} \beta_{\text {trig/R6 }}(38), v_{\mathrm{C} 12 \mathrm{C} 13}(8), v_{\mathrm{C} 14 \mathrm{C} 15}(8), \rho_{\mathrm{C} 13 \mathrm{H} 27}(8), \rho_{\mathrm{C} 15 \mathrm{H} 29}(7), \\
v_{\mathrm{C} 9 \mathrm{C} 10}(6), v_{\mathrm{C} 11 \mathrm{C} 17}(5), \rho_{\mathrm{C} 14 \mathrm{H} 28}(5)\end{array}$ \\
\hline 53 & 1141.7 & 6.8 & 3.7 & $v_{\mathrm{C} 2 \mathrm{~N} 3}(42), v_{\mathrm{C} 2 \mathrm{C} 20}(10), \rho_{\mathrm{C} 18 \mathrm{~N} 3}(6), v_{\mathrm{C} 8 \mathrm{C} 17}(6)$ \\
\hline 54 & 1175.9 & 6.6 & 4.4 & $\begin{array}{l}\rho_{\mathrm{C} 13 \mathrm{H} 27}(16), \rho_{\mathrm{C} 12 \mathrm{H} 26}(12), v_{\mathrm{C} 9 \mathrm{C} 16}(10), v_{\mathrm{C} 9 \mathrm{C} 10}(7),{ }^{1} \beta_{\text {trig/R6 }}(7), \\
{ }^{3} \beta_{\text {trig/R6}}(6)\end{array}$ \\
\hline 55 & 1184.0 & 17.5 & 0.4 & $\rho_{\mathrm{C} 15 \mathrm{H} 29}(32), \rho_{\mathrm{C} 14 \mathrm{H} 28}(10), \rho_{\mathrm{C} 13 \mathrm{H} 27}(10), v_{\mathrm{C} 14 \mathrm{C} 15}(9), v_{\mathrm{C} 13 \mathrm{C} 15}(6)$ \\
\hline 56 & 1199.1 & 25.7 & 8.6 & $\rho_{\mathrm{C} 6 \mathrm{H} 24}(30), \rho_{\mathrm{C} 7 \mathrm{H} 25}(13), v_{\mathrm{C} 6 \mathrm{C} 7}(8), \rho_{\mathrm{C} 13 \mathrm{H} 27}(6)$ \\
\hline 57 & 1215.9 & 2.5 & 4.5 & $\begin{array}{l}\rho_{\mathrm{C} 19 \mathrm{H} 30}(25), \rho_{\mathrm{C} 7 \mathrm{H} 25}(12), v_{\mathrm{C} 8 \mathrm{C} 19}(10), v_{\mathrm{C} 11 \mathrm{C} 17}(7), \rho_{\mathrm{C} 6 \mathrm{H} 24}(6), \\
\rho_{\mathrm{C} 14 \mathrm{H} 28}(6)\end{array}$ \\
\hline 58 & 1221.4 & 39.6 & 0.5 & $\tau_{\mathrm{C} 20 \mathrm{H} 31 \mathrm{H} 32}(51), \tau_{\mathrm{C}_{2} \mathrm{H} 22 \mathrm{H} 21}(10)$ \\
\hline 59 & 1256.8 & 77.0 & 2.2 & $\begin{array}{l}\tau_{\mathrm{C} 20 \mathrm{H} 31 \mathrm{H} 32}(13), \rho_{\mathrm{C} 12 \mathrm{H} 26}(11), v_{\mathrm{C} 7 \mathrm{C} 18}(8), \omega_{\mathrm{C} 20 \mathrm{H} 31 \mathrm{H} 32}(7), v_{\mathrm{C} 9 \mathrm{C} 10}(6), \\
\rho_{\mathrm{C} 14 \mathrm{H} 28}(5), v_{\mathrm{C} 11 \mathrm{C} 12}(5)\end{array}$ \\
\hline 60 & 1277.3 & 78.9 & 0.2 & $\omega_{\mathrm{C}_{2} \mathrm{H} 31 \mathrm{H} 32}(21), \tau_{\mathrm{C}_{2} \mathrm{H} 22 \mathrm{H} 21}(13), \rho_{\mathrm{C} 14 \mathrm{H} 28}(10),{ }^{2} \beta_{\text {trig/R }}(8), \rho_{\mathrm{C} 12 \mathrm{H} 26}(7)$ \\
\hline 61 & 1293.8 & 528.9 & 10.6 & $\begin{array}{l}v_{\mathrm{C} 9 \mathrm{C} 10}(12), \omega_{\mathrm{C}_{20} \mathrm{H} 31 \mathrm{H} 32}(11), v_{\mathrm{C} 9 \mathrm{C} 16}(9), v_{\mathrm{C} 11 \mathrm{C} 17}(6), v_{\mathrm{C} 8 \mathrm{C} 16}(6), \\
\tau_{\mathrm{C}_{2} \mathrm{H} 22 \mathrm{H} 21}(5), v_{\mathrm{C} 8 \mathrm{C} 17}(5)\end{array}$ \\
\hline 62 & 1317.6 & 120.0 & 1.9 & $\begin{array}{l}v_{\mathrm{C}_{11 \mathrm{C} 17}}(18), v_{\mathrm{C} 8 \mathrm{C} 17}(14), \rho_{\mathrm{C} 12 \mathrm{H} 26}(8), \rho_{\mathrm{C} 17 \mathrm{O} 4}(7),{ }^{2} \beta_{2 / R 6}(6), \\
\omega_{\mathrm{C}_{20 \mathrm{H} 31 \mathrm{H} 32}}(5)\end{array}$ \\
\hline 63 & 1333.4 & 156.0 & 4.2 & $\begin{array}{l}v_{\mathrm{N} 3 \mathrm{C} 18}(19), \rho_{\mathrm{N} 3 \mathrm{H} 23}(13), v_{\mathrm{C} 16 \mathrm{C} 18}(10), \omega_{\mathrm{C} 2 \mathrm{H} 22 \mathrm{H} 21}(8), \rho_{\mathrm{C} 6 \mathrm{H} 24}(7), \\
v_{\mathrm{C} 9 \mathrm{C} 16}(6), \tau_{\mathrm{C}_{2} \mathrm{H} 22 \mathrm{H} 21}(6)\end{array}$ \\
\hline 64 & 1340.7 & 26.0 & 2.5 & $\omega_{\mathrm{C} 20 \mathrm{H} 31 \mathrm{H} 32}(36), \tau_{\mathrm{C} 2 \mathrm{H} 22 \mathrm{H} 21}(32), v_{\mathrm{N} 3 \mathrm{C} 18}(8)$ \\
\hline 65 & 1358.8 & 14.2 & 0.9 & $\begin{array}{l}v_{\mathrm{C} 10 \mathrm{C} 11}(15), v_{\mathrm{C} 10 \mathrm{C} 14}(15), v_{\mathrm{C} 11 \mathrm{C} 12}(14), v_{\mathrm{C} 13 \mathrm{C} 15}(14), v_{\mathrm{C} 12 \mathrm{C} 13}(13), \\
v_{\mathrm{C} 14 \mathrm{C} 15}(13)\end{array}$ \\
\hline 66 & 1378.1 & 24.3 & 1.7 & $\begin{array}{l}v_{\mathrm{C} 16 \mathrm{C} 18}(18), v_{\mathrm{C} 8 \mathrm{C} 19}(11), v_{\mathrm{C} 9 \mathrm{C} 16}(10), v_{\mathrm{C} 8 \mathrm{C} 16}(9), v_{\mathrm{C} 6 \mathrm{C} 19}(7), v_{\mathrm{C} 6 \mathrm{C} 7}(6), \\
\rho_{\mathrm{C} 9 \mathrm{O} 5}(6), v_{\mathrm{C} 7 \mathrm{C} 18}(6), v_{\mathrm{C} 9 \mathrm{C} 10}(5)\end{array}$ \\
\hline 67 & 1395.3 & 37.1 & 2.1 & $\omega_{\mathrm{C} 2 \mathrm{H} 22 \mathrm{H} 21}(65), \delta_{1 / \mathrm{C}_{2} \mathrm{H} 22 \mathrm{H} 21}(5)$ \\
\hline 68 & 1435.1 & 33.0 & 0.5 & $\rho_{\mathrm{C} 7 \mathrm{H} 25}(24), v_{\mathrm{C} 6 \mathrm{C} 7}(11), v_{\mathrm{C} 8 \mathrm{C} 19}(10), v_{\mathrm{C} 8 \mathrm{C} 16}(7), v_{\mathrm{C} 9 \mathrm{C} 16}(6)$, \\
\hline
\end{tabular}




\begin{tabular}{|c|c|c|c|c|}
\hline & & & & $\delta_{1 / \mathrm{C}_{2} \mathrm{H}_{22 \mathrm{H}} 21}(5)$ \\
\hline 69 & 1468.5 & 7.8 & 1.2 & $\delta_{1 / \mathrm{C}_{20} \mathrm{H} 31 \mathrm{H} 32}(92)$ \\
\hline 70 & 1478.2 & 1.3 & 3.7 & $\begin{array}{l}\rho_{\mathrm{C} 15 \mathrm{H} 29}(14), \rho_{\mathrm{C} 13 \mathrm{H} 27}(12), \rho_{\mathrm{C} 19 \mathrm{H} 30}(7), v_{\mathrm{C} 10 \mathrm{C} 14}(7), v_{\mathrm{C} 8 \mathrm{C} 16}(6), \\
\delta_{1 / \mathrm{C} 2 \mathrm{H} 22 \mathrm{H} 21}(6), v_{\mathrm{C}_{11} \mathrm{C} 12}(6)\end{array}$ \\
\hline 71 & 1486.4 & 8.6 & 0.7 & $\begin{array}{l}\delta_{1 / \mathrm{C}_{22} \mathrm{H}_{22} \mathrm{H}_{21}}(17), \rho_{\mathrm{C} 13 \mathrm{H} 27}(8), \rho_{\mathrm{C} 15 \mathrm{H} 29}(8), \rho_{\mathrm{C} 19 \mathrm{H} 30}(7), \rho_{\mathrm{C} 6 \mathrm{H} 24}(6), \\
{ }^{2} \beta_{2 / R 6}(5), v_{\mathrm{C} 8 \mathrm{C} 16}(5)\end{array}$ \\
\hline 72 & 1501.1 & 5.4 & 1.7 & $\delta_{1 / \mathrm{C}_{2} \mathrm{H} 22 \mathrm{H} 21}(59)$ \\
\hline 73 & 1508.4 & 3.4 & 1.6 & $\rho_{\mathrm{C} 14 \mathrm{H} 28}(19), \rho_{\mathrm{C} 12 \mathrm{H} 26}(18), v_{\mathrm{C} 10 \mathrm{C} 11}(16), v_{\mathrm{C} 13 \mathrm{C} 15}(8)$ \\
\hline 74 & 1552.9 & 196.2 & 1.4 & $\rho_{\mathrm{N} 3 \mathrm{H} 23}(19), v_{\mathrm{C} 6 \mathrm{C} 19}(18), v_{\mathrm{N} 3 \mathrm{C} 18}(16), \rho_{\mathrm{C} 6 \mathrm{H} 24}(12), v_{\mathrm{C} 6 \mathrm{C} 7}(7), v_{\mathrm{C} 8 \mathrm{C} 16}(6)$ \\
\hline 75 & 1611.2 & 55.4 & 12.5 & $\begin{array}{l}v_{\mathrm{C} 13 \mathrm{C} 15}(9), v_{\mathrm{C} 10 \mathrm{C} 11}(9), \rho_{\mathrm{N} 3 \mathrm{H} 23}(8), v_{\mathrm{C} 6 \mathrm{C} 19}(8), v_{\mathrm{C} 8 \mathrm{C} 19}(8), v_{\mathrm{C} 16 \mathrm{C} 18}(7), \\
v_{\mathrm{O} 5 \mathrm{C} 9}(7)\end{array}$ \\
\hline 76 & 1613.5 & 77.7 & 3.0 & $\rho_{\mathrm{N} 3 \mathrm{H} 23}(17), v_{\mathrm{C} 13 \mathrm{C} 15}(10), v_{\mathrm{C} 10 \mathrm{C} 11}(10), v_{\mathrm{C} 6 \mathrm{C} 19}(6), v_{\mathrm{C} 16 \mathrm{C} 18}(5)$ \\
\hline 77 & 1626.5 & 31.1 & 17.4 & $\begin{array}{l}v_{\mathrm{C} 14 \mathrm{C} 15}(13), v_{\mathrm{C} 11 \mathrm{C} 12}(10), v_{\mathrm{O} 5 \mathrm{C} 9}(9), v_{\mathrm{C} 6 \mathrm{C} 7}(7),{ }^{3} \beta_{2 / R 6}(7), v_{\mathrm{C} 12 \mathrm{C} 13}(5), \\
v_{\mathrm{C} 8 \mathrm{C} 19}(5)\end{array}$ \\
\hline 78 & 1630.7 & 261.2 & 5.1 & $\begin{array}{l}v_{\mathrm{C} 6 \mathrm{C} 7}(14), v_{\mathrm{C} 14 \mathrm{C} 15}(7), v_{\mathrm{C} 8 \mathrm{C} 19}(6), v_{\mathrm{C} 12 \mathrm{C} 13}(6), v_{\mathrm{O} 4 \mathrm{C} 17}(6),{ }^{1} \beta_{3 / R 6}(6), \\
v_{\mathrm{C} 11 \mathrm{C} 12}(6), \rho_{\mathrm{C} 7 \mathrm{H} 25}(5)\end{array}$ \\
\hline 79 & 1669.7 & 208.8 & 28.6 & $v_{\mathrm{O} 5 \mathrm{C} 9}(43),{ }^{2} \beta_{3 / R 6}(6),{ }^{2} \beta_{\text {trig/R6 }}(6)$ \\
\hline 80 & 1721.1 & 179.5 & 29.1 & $v_{\mathrm{O} 4 \mathrm{C} 17}(71),{ }^{2} \beta_{\text {trig/R6 }}(9)$ \\
\hline 81 & 2670.3 & 1.8 & 8.2 & $v_{\mathrm{S} 1 \mathrm{H} 33}(100)$ \\
\hline 82 & 3017.6 & 47.4 & 8.0 & $v_{\mathrm{C}_{2} \mathrm{H} 21}(91), v_{\mathrm{C}_{2} \mathrm{H} 22}(8)$ \\
\hline 83 & 3060.3 & 18.9 & 11.6 & $v_{\mathrm{C} 20 \mathrm{H} 32}(52), v_{\mathrm{C} 20 \mathrm{H} 31}(44)$ \\
\hline 84 & 3087.1 & 6.0 & 4.5 & $v_{\mathrm{C}_{2} \mathrm{H} 22}(78), v_{\mathrm{C}_{20} \mathrm{H} 32}(11), v_{\mathrm{C}_{2} \mathrm{H} 21}(9)$ \\
\hline 85 & 3111.7 & 22.3 & 4.3 & $v_{\mathrm{C} 20 \mathrm{H} 31}(53), v_{\mathrm{C}_{20} \mathrm{H} 32}(36), v_{\mathrm{C}_{2} \mathrm{H} 22}(10)$ \\
\hline 86 & 3170.3 & 3.4 & 4.5 & $v_{\mathrm{C} 15 \mathrm{H} 29}(49), v_{\mathrm{C} 13 \mathrm{H} 27}(45)$ \\
\hline 87 & 3172.5 & 11.3 & 7.9 & $v_{\mathrm{C} 6 \mathrm{H} 24}(90), v_{\mathrm{C} 7 \mathrm{H} 25}(6)$ \\
\hline 88 & 3185.0 & 13.0 & 14.6 & $v_{\mathrm{C} 13 \mathrm{H} 27}(43), v_{\mathrm{C} 15 \mathrm{H} 29}(42), v_{\mathrm{C} 12 \mathrm{H} 26}(9), v_{\mathrm{C} 14 \mathrm{H} 28}(5)$ \\
\hline 89 & 3201.7 & 3.7 & 7.0 & $v_{\mathrm{C}_{12} \mathrm{H} 26}(81), v_{\mathrm{C} 13 \mathrm{H} 27}(9), v_{\mathrm{C} 14 \mathrm{H} 28}(9)$ \\
\hline 90 & 3206.0 & 9.7 & 11.2 & $v_{\mathrm{C} 14 \mathrm{H} 28}(83), v_{\mathrm{C} 15 \mathrm{H} 29}(8), v_{\mathrm{C} 12 \mathrm{H} 26}(6)$ \\
\hline 91 & 3206.7 & 9.0 & 9.0 & $v_{\mathrm{C} 7 \mathrm{H} 25}(89), v_{\mathrm{C} 19 \mathrm{H} 30}(7)$ \\
\hline 92 & 3213.1 & 4.7 & 13.3 & $v_{\mathrm{C} 19 \mathrm{H} 30}(90)$ \\
\hline 93 & 3467.2 & 99.1 & 30.5 & $v_{\mathrm{N} 3 \mathrm{H} 23}(99)$ \\
\hline
\end{tabular}


Table S.13. Frequencies of normal vibrations, IR and Raman intensities and PEDs for TTT conformer calculated at the B3LYP/6-311++G(d,p) level of theory.

\begin{tabular}{|c|c|c|c|c|}
\hline $\mathrm{Nr}$ & $\begin{array}{l}\text { Freq. } \\
{\left[\mathrm{cm}^{-1}\right]}\end{array}$ & IR Int. & $\begin{array}{c}\text { Raman } \\
\text { Int. }\end{array}$ & PED (contributions $>5 \%$ ) \\
\hline 1 & 25.6 & 0.8 & 10.2 & $\tau_{\mathrm{C}_{2} \mathrm{C} 20}(21), \tau_{\mathrm{C}_{2} \mathrm{~N} 3}(21), \tau_{\mathrm{S} 1 \mathrm{C} 20_{2}}(21), \tau_{\mathrm{N} 3 \mathrm{C} 18}(20)$ \\
\hline 2 & 39.7 & 1.6 & 0.4 & ${ }^{2} \tau_{3 / R 6}(37),{ }^{1} \tau_{3 / R 6}(15), \tau_{\mathrm{C}_{2 N} 3}(14), \omega_{\mathrm{N} 3 \mathrm{H} 23}(8),{ }^{2} \tau_{2 / R 6}(7)$ \\
\hline 3 & 51.8 & 8.6 & 1.0 & $\tau_{\mathrm{C}_{2} \mathrm{~N} 3}(33), \omega_{\mathrm{N} 3 \mathrm{H} 23}(24), \tau_{\mathrm{C}_{2} \mathrm{C}_{20}}(15),{ }^{2} \tau_{2 / R 6}(11),{ }^{2} \tau_{3 / R \sigma}(7)$ \\
\hline 4 & 60.5 & 0.5 & 1.4 & $\beta_{\mathrm{C}_{18 \mathrm{~N}_{3} \mathrm{C}_{2}}}(32), \delta_{2 / \mathrm{C}_{2} \mathrm{H} 22 \mathrm{H} 21}(31), \delta_{2 / \mathrm{C}_{20} \mathrm{H}_{31} \mathrm{H}_{32}}(15), \rho_{\mathrm{C}_{18 \mathrm{~N} 3}}(14)$ \\
\hline 5 & 75.7 & 6.3 & 0.0 & $\tau_{\mathrm{S}_{1} \mathrm{C} 20}(29), \tau_{\mathrm{C}_{2} \mathrm{~N} 3}(17), \tau_{\mathrm{C}_{2} \mathrm{C} 20}(12), \omega_{\mathrm{N} 3 \mathrm{H} 23}(10), \tau_{\mathrm{N} 3 \mathrm{C} 18}(9)$ \\
\hline 6 & 96.4 & 12.9 & 0.2 & $\tau_{\mathrm{S}_{1} \mathrm{C} 20}(31), \omega_{\mathrm{N} 3 \mathrm{H} 23}(27),{ }^{2} \tau_{2 / R 6}(18), \tau_{\mathrm{C}_{2} \mathrm{C} 20}(8)$ \\
\hline 7 & 121.0 & 1.6 & 0.7 & ${ }^{2} \tau_{1 / R \sigma}(43),{ }^{3} \tau_{2 / R 6}(16),{ }^{2} \tau_{2 / R 6}(11),{ }^{3} \tau_{3 / R 6}(8)$ \\
\hline 8 & 151.5 & 0.4 & 0.9 & $\begin{array}{l}\omega_{\mathrm{N} 3 \mathrm{H} 23}(32), \tau_{\mathrm{C} 2 \mathrm{C} 20}(20), \tau_{\mathrm{S} 1 \mathrm{C} 20}(16),{ }^{2} \tau_{1 / R 6}(8),{ }^{2} b f l_{R R}(6), \\
\rho_{\mathrm{C} 20 \mathrm{H} 31 \mathrm{H} 32}(5)\end{array}$ \\
\hline 9 & 169.0 & 0.5 & 0.0 & $\begin{array}{l}{ }^{4} b f l_{R R}(26),{ }^{2} b f l_{R R}(18), \tau_{N 3 C l 8}(15),{ }^{2} \tau_{3 / R 6}(8), \omega_{C 17 O 4}(6), \omega_{C 9 O S}(5), \\
{ }^{1} \tau_{3 / R 6}(5)\end{array}$ \\
\hline 10 & 173.0 & 0.5 & 0.7 & $\delta_{2 / \mathrm{C} 20 \mathrm{H} 31 \mathrm{H} 32}(43), v_{\mathrm{C} 2 \mathrm{~N} 3}(8), \rho_{\mathrm{C} 18 \mathrm{~N} 3}(8)$ \\
\hline 11 & 201.8 & 0.0 & 0.9 & ${ }^{1} \tau_{3 / R 6}(27),{ }^{1} \tau_{2 / R 6}(23),{ }^{4} b f l_{R R}(15), \omega_{C l 9 H 30}(6)$ \\
\hline 12 & 229.0 & 1.8 & 0.6 & $\begin{array}{l}\delta_{2 / \mathrm{C}_{2} \mathrm{H} 22 \mathrm{H} 21}(16),{ }^{1} \beta_{3 / R \sigma}(14),{ }^{2} \beta_{2 / R 6}(11), v_{\mathrm{C} 8 \mathrm{C} 17}(9), v_{\mathrm{S}_{1} \mathrm{C} 20}(6), \\
{ }^{3} \beta_{2 / R 6}(6),{ }^{1} \beta_{\text {trig/R6 }}(5)\end{array}$ \\
\hline 13 & 267.8 & 0.5 & 0.7 & $\begin{array}{l}{ }^{2} b f l_{R R}(30), \omega_{C 18 N 3}(10), \omega_{N 3 H 23}(10),{ }^{l} \tau_{2 / R 6}(8), \omega_{C 7 H 25}(6),{ }^{l} \tau_{3 / R 6}(6), \\
{ }^{4} b l_{R R}(6),{ }^{l} \tau_{1 / R 6}(5)\end{array}$ \\
\hline 14 & 295.2 & 0.3 & 0.7 & $\delta_{2 / \mathrm{C} 2 \mathrm{H} 22 \mathrm{H} 21}(18), \delta_{2 / \mathrm{C} 20 \mathrm{H} 31 \mathrm{H} 32}(18), \rho_{\mathrm{C} 18 \mathrm{~N} 3}(15), \beta_{\mathrm{C} 18 \mathrm{~N} 3 \mathrm{C} 2_{2}}(14)$ \\
\hline 15 & 341.0 & 1.1 & 0.1 & ${ }^{2} \beta_{2 / R 6}(30), \rho_{\mathrm{C} 17 \mathrm{O} 4}(21),{ }^{2} \beta_{3 / R 6}(18), \rho_{\mathrm{C} 18 \mathrm{~N} 3}(7), \delta_{2 / \mathrm{C}_{20 \mathrm{H} 31 \mathrm{H} 32}}(6)$ \\
\hline 16 & 353.5 & 1.5 & 0.3 & $\begin{array}{l}{ }^{2} \beta_{3 / R 6}(18),{ }^{2} \beta_{2 / R 6}(13), v_{\mathrm{C} 9 \mathrm{C} 10}(9), \delta_{2 / \mathrm{C}_{20 \mathrm{H} 31 \mathrm{H} 32}}(8), v_{\mathrm{C} 8 \mathrm{C} 17}(8), \\
v_{\mathrm{C} 11 \mathrm{C} 17}(7), v_{\mathrm{C} 16 \mathrm{C} 18}(5)\end{array}$ \\
\hline 17 & 402.9 & 11.5 & 0.6 & $\begin{array}{l}{ }^{2} \beta_{3 / R \sigma}(18),{ }^{1} \beta_{2 / R \sigma}(9),{ }^{2} \beta_{2 / R}(9), \delta_{2 / \mathrm{C} 2 \mathrm{H} 22 \mathrm{H} 21}(6), v_{\mathrm{S}_{1} \mathrm{C}_{20}}(6), \rho_{\mathrm{C} 18 \mathrm{~N} 3}(5), \\
v_{\mathrm{C}_{11} \mathrm{C} 17}(5)\end{array}$ \\
\hline 18 & 426.5 & 21.4 & 0.6 & $\rho_{\mathrm{C} 9 \mathrm{O} 5}(42), \rho_{\mathrm{C} 17 \mathrm{O} 4}(16), v_{\mathrm{C} 9 \mathrm{C} 16}(10),{ }^{1} \beta_{3 / R 6}(9)$ \\
\hline 19 & 428.0 & 0.5 & 0.0 & ${ }^{3} \tau_{3 / R 6}(59),{ }^{4} b f l_{R R}(15),{ }^{3} \tau_{2 / R \sigma}(8)$ \\
\hline 20 & 433.5 & 0.1 & 0.0 & ${ }^{3} \tau_{2 / R 6}(29),{ }^{1} \tau_{2 / R 6}(21),{ }^{1} \tau_{3 / R 6}(17),{ }^{2} \tau_{1 / R 6}(7), \omega_{\mathrm{C} 9 \mathrm{O} 5}(6)$ \\
\hline 21 & 479.0 & 3.3 & 8.8 & ${ }^{2} \beta_{2 / R 6}(32),{ }^{2} \beta_{3 / R 6}(18),{ }^{3} \beta_{3 / R 6}(10), v_{\mathrm{C} 8 \mathrm{C} 16}(7), v_{\mathrm{C} 11 \mathrm{C} 17}(6),{ }^{1} \beta_{3 / R 6}(6)$ \\
\hline 22 & 490.1 & 2.1 & 0.0 & ${ }^{1} \tau_{3 / R \sigma}(23),{ }^{3} \tau_{2 / R \sigma}(23),{ }^{1} \tau_{2 / R \sigma}(11),{ }^{2} \tau_{2 / R 6}(10),{ }^{3} \tau_{3 / R 6}(5),{ }^{1} \tau_{1 / R \sigma}(5)$ \\
\hline 23 & 495.4 & 5.4 & 3.7 & $\begin{array}{l}{ }^{1} \beta_{2 / R 6}(17), \rho_{\mathrm{C} 18 \mathrm{~N} 3}(16),{ }^{3} \beta_{2 / R 6}(11),{ }^{2} \beta_{3 / R 6}(10),{ }^{1} \beta_{3 / R 6}(6), \\
\beta_{\mathrm{C} 18 \mathrm{~N} 3 \mathrm{C} 2}(5)\end{array}$ \\
\hline 24 & 556.1 & 7.3 & 0.0 & $\omega_{\mathrm{C} 18 \mathrm{~N} 3}(30),{ }^{1} \tau_{3 / R \sigma}(14),{ }^{\mathrm{I}}{ }_{\tau_{2 / R \sigma}}(13),{ }^{\mathrm{I}} \tau_{1 / R \sigma}(10),{ }^{2} b f l_{R R}(6)$ \\
\hline 25 & 612.8 & 3.3 & 1.1 & ${ }^{1} \beta_{2 / R 6}(20),{ }^{3} \beta_{2 / R 6}(15),{ }^{1} \beta_{3 / R \sigma}(15),{ }^{2} \beta_{\text {trig/R6}}(13),{ }^{3} \beta_{3 / R \sigma}(6)$ \\
\hline 26 & 633.5 & 47.2 & 0.1 & $\tau_{\mathrm{N} 3 \mathrm{C} 18}(46), \omega_{\mathrm{N} 3 \mathrm{H} 23}(31), \tau_{\mathrm{C} 2 \mathrm{~N} 3}(6)$ \\
\hline 27 & 636.1 & 0.1 & 1.1 & $\begin{array}{l}{ }^{2} \beta_{\text {trig/R6 }}(36),{ }^{1} \beta_{2 / R 6}(10),{ }^{1} \beta_{3 / R 6}(10),{ }^{3} \beta_{3 / R 6}(9), \rho_{\mathrm{C} 18 \mathrm{~N}_{3}}(7), \\
\beta_{\mathrm{C} 18 \mathrm{~N} 3 \mathrm{C} 2}(7)\end{array}$ \\
\hline 28 & 673.0 & 6.6 & 0.0 & ${ }^{3} \tau_{1 / R 6}(38),{ }^{1} \tau_{1 / R 6}(19), \omega_{\mathrm{C} 9 \mathrm{O} 5}(12), \omega_{\mathrm{C} 18 \mathrm{~N} 3}(11)$ \\
\hline 29 & 678.3 & 8.5 & 1.4 & ${ }^{3} \beta_{3 / R 6}(40),{ }^{3} \beta_{2 / R 6}(8), v_{\mathrm{C} 10 \mathrm{C} 11}(7), v_{\mathrm{S} 1 \mathrm{C} 20}(6)$ \\
\hline 30 & 706.4 & 1.0 & 0.2 & $\rho_{\mathrm{C} 9 \mathrm{O} 5}(19),{ }^{3} \beta_{2 / R 6}(18), \rho_{\mathrm{C} 17 \mathrm{O} 4}(18),{ }^{1} \beta_{2 / R 6}(10),{ }^{1} \beta_{3 / R 6}(8)$ \\
\hline 31 & 724.9 & 34.9 & 0.0 & ${ }^{1} \tau_{1 / R 6}(29),{ }^{3} \tau_{1 / R 6}(27), \omega_{\mathrm{C} 6 \mathrm{H} 24}(9), \omega_{\mathrm{C} 15 \mathrm{H} 29}(6), \omega_{\mathrm{C} 17 \mathrm{O} 4}(6)$ \\
\hline 32 & 746.0 & 54.1 & 0.0 & $\begin{array}{l}{ }_{{ }^{1}} \tau_{1 / R 6}(14), \omega_{\mathrm{C} 18 \mathrm{~N} 3}(14), \omega_{\mathrm{C} 13 \mathrm{H} 27}(13), \omega_{\mathrm{C} 9 \mathrm{O} 5}(11), \omega_{\mathrm{C} 17 \mathrm{O} 4}(8), \\
\omega_{\mathrm{C} 15 \mathrm{H} 29}(7), \omega_{\mathrm{C} 14 \mathrm{H} 28}(7)\end{array}$ \\
\hline 33 & 759.8 & 4.2 & 1.2 & $v_{\mathrm{S}_{1} \mathrm{C}_{20}}(52),{ }^{\mathrm{1}} \beta_{3 / R \sigma}(9), \delta_{2 / \mathrm{C}_{2} \mathrm{H} 22 \mathrm{H} 21}(9),{ }^{3} \beta_{2 / R \sigma}(5)$ \\
\hline 34 & 786.2 & 10.7 & 0.0 & $\begin{array}{l}{ }^{3} \tau_{1 / R 6}(19), \omega_{\mathrm{C} 7 \mathrm{H} 25}(13),{ }^{2} \tau_{1 / R 6}(12), \omega_{\mathrm{C} 19 \mathrm{H} 30}(10), \omega_{\mathrm{C} 6 \mathrm{H} 24}(9), \\
\omega_{\mathrm{C} 17 \mathrm{O} 4}(9), \omega_{\mathrm{C} 9 \mathrm{O} 5}(9)\end{array}$ \\
\hline 35 & 803.7 & 1.7 & 1.1 & ${ }^{1} \beta_{\text {trig/R } 6}(17),{ }^{3} \beta_{2 / R \sigma}(10),{ }^{3} \beta_{\text {trig/R }}(9),{ }^{2} \beta_{\text {trig/R6 }}(9),{ }_{2 / R \sigma}^{1}(8)$, \\
\hline
\end{tabular}




\begin{tabular}{|c|c|c|c|c|}
\hline & & & & ${ }^{1} \beta_{3 / R 6}(8)$ \\
\hline 36 & 805.6 & 0.9 & 0.0 & $\rho_{\mathrm{C} 20 \mathrm{H} 31 \mathrm{H} 32}(25), \rho_{\mathrm{C} 2 \mathrm{H} 22 \mathrm{H} 21}(14), \omega_{\mathrm{C} 9 \mathrm{O} 5}(13),{ }^{1} \tau_{1 / R 6}(9),{ }^{2} \tau_{1 / R 6}(9)$ \\
\hline 37 & 809.9 & 3.7 & 0.1 & $\begin{array}{l}\omega_{\mathrm{C} 9 \mathrm{O} 5}(18), \omega_{\mathrm{C}_{13} \mathrm{H} 27}(13), \rho_{\mathrm{C}_{20} \mathrm{H} 31 \mathrm{H} 32}(11), \omega_{\mathrm{C} 15 \mathrm{H} 29}(10), \rho_{\mathrm{C} 2 \mathrm{H} 22 \mathrm{H} 21}(6), \\
\omega_{\mathrm{C} 18 \mathrm{~N} 3}(5),{ }^{1} \tau_{1 / R 6}(5)\end{array}$ \\
\hline 38 & 828.8 & 24.0 & 0.0 & $\begin{array}{l}\omega_{\mathrm{C} 7 \mathrm{H} 25}(22), \omega_{\mathrm{C} 17 \mathrm{O} 4}(16),{ }^{1} \tau_{1 / R 6}(15), \omega_{\mathrm{C} 18 \mathrm{~N} 3}(9), \omega_{\mathrm{C} 6 \mathrm{H} 24}(7), \\
{ }^{2} \tau_{1 / R 6}(6), \omega_{\mathrm{C} 15 \mathrm{H} 29}(5)\end{array}$ \\
\hline 39 & 872.6 & 3.7 & 0.6 & $\beta_{\mathrm{C}_{20 \mathrm{~S} 1 \mathrm{H} 33}}(64), v_{\mathrm{C}_{2} \mathrm{C} 20}(12)$ \\
\hline 40 & 898.2 & 33.2 & 3.0 & $\rho_{\mathrm{C} 17 \mathrm{O} 4}(12), v_{\mathrm{C} 16 \mathrm{C} 18}(10), v_{\mathrm{C} 7 \mathrm{C} 18}(10), v_{\mathrm{C}_{2} \mathrm{C} 20}(6), v_{\mathrm{N} 3 \mathrm{C} 18}(5)$ \\
\hline 41 & 911.0 & 0.5 & 0.0 & $\omega_{\mathrm{C} 19 \mathrm{H} 30}(31), \omega_{\mathrm{C} 7 \mathrm{H} 25}(22), \omega_{\mathrm{C} 12 \mathrm{H} 26}(12), \omega_{\mathrm{C} 14 \mathrm{H} 28}(12), \omega_{\mathrm{C} 15 \mathrm{H} 29}(6)$ \\
\hline 42 & 917.8 & 0.5 & 0.0 & $\begin{array}{l}\omega_{\mathrm{C} 14 \mathrm{H} 28}(19), \omega_{\mathrm{C} 19 \mathrm{H} 30}(18), \omega_{\mathrm{C} 12 \mathrm{H} 26_{2}}(17), \omega_{\mathrm{C} 7 \mathrm{H} 25}(12), \omega_{\mathrm{C}_{13} \mathrm{H} 27}(10), \\
\omega_{\mathrm{C} 15 \mathrm{H} 29}(7)\end{array}$ \\
\hline 43 & 929.5 & 2.9 & 1.3 & ${ }^{1} \beta_{\text {trig/R6}}(25),{ }^{3} \beta_{\text {trig/R6 }}(23), \rho_{\mathrm{C} 9 \mathrm{O} 5}(14), v_{\mathrm{C} 16 \mathrm{C} 18}(7)$ \\
\hline 44 & 1000.2 & 0.0 & 0.0 & $\omega_{\mathrm{C} 6 \mathrm{H} 24}(48), \omega_{\mathrm{C} 19 \mathrm{H} 30}(20), \omega_{\mathrm{C} 7 \mathrm{H} 25}(10),{ }^{1} \tau_{1 / R 6}(7)$ \\
\hline 45 & 1002.8 & 2.1 & 0.0 & $\omega_{\mathrm{C} 12 \mathrm{H} 26}(29), \omega_{\mathrm{C} 14 \mathrm{H} 28}(29), \omega_{\mathrm{C} 15 \mathrm{H} 29}(11), \omega_{\mathrm{C} 13 \mathrm{H} 27}(9), \omega_{\mathrm{C} 6 \mathrm{H} 24}(7)$ \\
\hline 46 & 1014.9 & 86.6 & 3.5 & $\begin{array}{l}v_{\mathrm{C} 8 \mathrm{C} 17}(15), v_{\mathrm{C} 2 \mathrm{~N} 3}(10), v_{\mathrm{C} 11 \mathrm{C} 12}(7), v_{\mathrm{C} 13 \mathrm{C} 15}(6), v_{\mathrm{C} 8 \mathrm{C} 19}(6), \rho_{\mathrm{C} 17 \mathrm{O} 4}(6), \\
v_{\mathrm{N} 3 \mathrm{C} 18}(5)\end{array}$ \\
\hline 47 & 1017.1 & 0.0 & 0.0 & $\omega_{\mathrm{C} 13 \mathrm{H} 27}(29), \omega_{\mathrm{C} 15 \mathrm{H} 29}(29), \omega_{\mathrm{C} 12 \mathrm{H} 26}(15), \omega_{\mathrm{C} 14 \mathrm{H} 28}(13),{ }^{3} \tau_{1 / R 6}(12)$ \\
\hline 48 & 1032.9 & 2.2 & 0.1 & $\tau_{\mathrm{C} 20 \mathrm{H} 31 \mathrm{H} 32}(37), \rho_{\mathrm{C} 2 \mathrm{H} 22 \mathrm{H} 21}(35), \rho_{\mathrm{C} 20 \mathrm{H} 31 \mathrm{H} 32}(26)$ \\
\hline 49 & 1058.3 & 0.3 & 4.8 & $v_{\mathrm{C} 13 \mathrm{C} 15}(33), v_{\mathrm{C} 14 \mathrm{C} 15}(13), \rho_{\mathrm{C} 14 \mathrm{H} 28}(12), \rho_{\mathrm{C} 12 \mathrm{H} 26}(11), v_{\mathrm{C} 12 \mathrm{C} 13}(10)$ \\
\hline 50 & 1086.0 & 2.6 & 0.3 & $v_{\mathrm{C}_{2} \mathrm{C} 20}(60), \beta_{\mathrm{C}_{20} \mathrm{~S}_{1} \mathrm{H} 33}(14), \delta_{2 / \mathrm{C}_{2} \mathrm{H} 22 \mathrm{H} 21}(5)$ \\
\hline 51 & 1096.3 & 22.0 & 1.6 & $v_{\mathrm{C} 6 \mathrm{C} 19}(31), \rho_{\mathrm{C} 19 \mathrm{H} 30}(16),{ }^{1} \beta_{\text {trig/R6 }}(14), v_{\mathrm{C} 6 \mathrm{C} 7}(8), v_{\mathrm{C} 2 \mathrm{~N} 3}(6), \rho_{\mathrm{C} 7 \mathrm{H} 25}(6)$ \\
\hline 52 & 1113.5 & 1.2 & 0.3 & $\begin{array}{l}{ }^{3} \beta_{\text {trig/R6 }}(38), v_{\mathrm{C} 12 \mathrm{C} 13}(9), v_{\mathrm{C} 14 \mathrm{C} 15}(8), \rho_{\mathrm{C} 13 \mathrm{H} 27}(7), \rho_{\mathrm{C} 15 \mathrm{H} 29}(7), \\
v_{\mathrm{C} 9 \mathrm{C} 10}(6), v_{\mathrm{C} 11 \mathrm{C} 17}(5), \rho_{\mathrm{C} 12 \mathrm{H} 26}(5), \rho_{\mathrm{C} 14 \mathrm{H} 28}(5)\end{array}$ \\
\hline 53 & 1146.5 & 11.6 & 1.3 & $v_{\mathrm{C}_{2 N} 3}(49), \rho_{\mathrm{C} 18 \mathrm{~N} 3}(6), v_{\mathrm{C}_{8} \mathrm{C}_{17}}(5)$ \\
\hline 54 & 1177.2 & 3.2 & 5.0 & $\rho_{\mathrm{C} 13 \mathrm{H} 27}(19), \rho_{\mathrm{C} 12 \mathrm{H} 26}(13), v_{\mathrm{C} 9 \mathrm{C} 16}(8), v_{\mathrm{C} 9 \mathrm{C} 10}(7),{ }^{1} \beta_{\text {trig/R6}}(5)$ \\
\hline 55 & 1185.3 & 14.0 & 0.8 & $\begin{array}{l}\rho_{\mathrm{C} 15 \mathrm{H} 29}(29), v_{\mathrm{C} 14 \mathrm{C} 15}(10), \rho_{\mathrm{C} 14 \mathrm{H} 28}(8),{ }^{3} \beta_{\text {trig/R6 }}(7),{ }^{1} \beta_{\text {trig/R6 }}(6), \\
v_{\mathrm{C} 9 \mathrm{C} 16}(6), \rho_{\mathrm{C} 13 \mathrm{H} 27}(6)\end{array}$ \\
\hline 56 & 1198.1 & 37.3 & 8.6 & $\rho_{\mathrm{C} 6 \mathrm{H} 24}(31), \rho_{\mathrm{C} 7 \mathrm{H} 25}(15), v_{\mathrm{C} 6 \mathrm{C} 7}(8), \rho_{\mathrm{C} 13 \mathrm{H} 27}(6)$ \\
\hline 57 & 1208.0 & 0.3 & 0.0 & $\tau_{\mathrm{C} 20 \mathrm{H} 31 \mathrm{H} 32}(44), \tau_{\mathrm{C} 2 \mathrm{H} 22 \mathrm{H} 21}(27), \rho_{\mathrm{C} 2 \mathrm{H} 22 \mathrm{H} 21}(24)$ \\
\hline 58 & 1216.0 & 1.8 & 4.8 & $\rho_{\mathrm{C} 19 \mathrm{H} 30}(26), \rho_{\mathrm{C} 7 \mathrm{H} 25}(9), v_{\mathrm{C} 8 \mathrm{C} 19}(9), v_{\mathrm{C} 11 \mathrm{C} 17}(8), \rho_{\mathrm{C} 14 \mathrm{H} 28}(7), \rho_{\mathrm{C} 6 \mathrm{H} 24}(5)$ \\
\hline 59 & 1259.1 & 41.4 & 4.2 & $\begin{array}{l}\rho_{\mathrm{C} 12 \mathrm{H} 26}(14), v_{\mathrm{C} 7 \mathrm{C} 18}(10), \rho_{\mathrm{C} 14 \mathrm{H} 28}(7), \omega_{\mathrm{C} 20 \mathrm{H} 31 \mathrm{H} 32}(7), v_{\mathrm{C} 11 \mathrm{C} 12}(6), \\
{ }^{2} \beta_{\text {trig/R6}}(6), v_{\mathrm{C} 9 \mathrm{C} 10}(6), \rho_{\mathrm{C} 7 \mathrm{H} 25}(6)\end{array}$ \\
\hline 60 & 1281.5 & 0.0 & 1.2 & $\omega_{\mathrm{C} 20 \mathrm{H} 31 \mathrm{H} 32}(49), \omega_{\mathrm{C} 2 \mathrm{H} 22 \mathrm{H} 21}(10),{ }^{2} \beta_{\text {trig/R6 }}(5), \rho_{\mathrm{C} 14 \mathrm{H} 28}(5)$ \\
\hline 61 & 1291.1 & 743.7 & 7.8 & $\begin{array}{l}v_{\mathrm{C} 9 \mathrm{C} 10}(12), \omega_{\mathrm{C} 20 \mathrm{H} 31 \mathrm{H} 32}(11), v_{\mathrm{C} 9 \mathrm{C} 16}(8), v_{\mathrm{C} 8 \mathrm{C} 16}(7), v_{\mathrm{C} 7 \mathrm{C} 18}(6), \\
v_{\mathrm{C} 8 \mathrm{C} 17}(6), \rho_{\mathrm{C} 14 \mathrm{H} 28}(6), v_{\mathrm{C} 11 \mathrm{C} 17}(5)\end{array}$ \\
\hline 62 & 1317.4 & 0.2 & 0.9 & $\tau_{\mathrm{C} 2 \mathrm{H} 22 \mathrm{H} 21}(71), \tau_{\mathrm{C} 20 \mathrm{H} 31 \mathrm{H} 32}(21), \rho_{\mathrm{C} 20 \mathrm{H} 31 \mathrm{H} 32}(5)$ \\
\hline 63 & 1317.9 & 108.0 & 1.3 & $v_{\mathrm{C} 11 \mathrm{C} 17}(19), v_{\mathrm{C} 8 \mathrm{C} 17}(14), \rho_{\mathrm{C} 12 \mathrm{H} 26}(9), \rho_{\mathrm{C} 17 \mathrm{O} 4}(8),{ }^{2} \beta_{2 / R 6}(7), \rho_{\mathrm{C} 13 \mathrm{H} 27}(5)$ \\
\hline 64 & 1338.8 & 104.6 & 5.6 & $\begin{array}{l}v_{\mathrm{N} 3 \mathrm{C} 18}(20), \rho_{\mathrm{N} 3 \mathrm{H} 23}(15), v_{\mathrm{C} 16 \mathrm{C} 18}(11), \rho_{\mathrm{C} 6 \mathrm{H} 24}(7), \omega_{\mathrm{C} 20 \mathrm{H} 31 \mathrm{H} 32}(7), \\
v_{\mathrm{C} 9 \mathrm{C} 16}(6), \omega_{\mathrm{C} 2 \mathrm{H} 22 \mathrm{H} 21}(6)\end{array}$ \\
\hline 65 & 1358.6 & 17.2 & 0.9 & $\begin{array}{l}v_{\mathrm{C} 10 \mathrm{C} 14}(15), v_{\mathrm{C} 10 \mathrm{C} 11}(15), v_{\mathrm{C} 11 \mathrm{C} 12}(14), v_{\mathrm{C} 13 \mathrm{C} 15}(14), v_{\mathrm{C} 12 \mathrm{C} 13}(13), \\
v_{\mathrm{C} 14 \mathrm{C} 15}(13)\end{array}$ \\
\hline 66 & 1376.7 & 10.4 & 3.2 & $\begin{array}{l}v_{\mathrm{C} 16 \mathrm{C} 18}(13), v_{\mathrm{C} 8 \mathrm{C} 16}(11), v_{\mathrm{C} 8 \mathrm{C} 19}(10), v_{\mathrm{C} 9 \mathrm{C} 16}(9), v_{\mathrm{C} 6 \mathrm{C} 19}(7), v_{\mathrm{C} 9 \mathrm{C} 10}(6), \\
\rho_{\mathrm{C} 95}(6), v_{\mathrm{C} 7 \mathrm{C} 18}(5),{ }_{2 / R 6}(5)\end{array}$ \\
\hline 67 & 1410.8 & 5.2 & 0.9 & $\omega_{\mathrm{C}_{2} \mathrm{H} 22 \mathrm{H} 21}(56), \omega_{\mathrm{C} 20 \mathrm{H} 31 \mathrm{H} 32}(6), v_{\mathrm{C}_{2} \mathrm{C} 20}(6)$ \\
\hline 68 & 1435.7 & 56.0 & 0.2 & $\begin{array}{l}\rho_{\mathrm{C} 7 \mathrm{H} 25}(22), v_{\mathrm{C} 6 \mathrm{C} 7}(10), v_{\mathrm{C} 8 \mathrm{C} 19}(10), v_{\mathrm{C} 8 \mathrm{C} 16}(7), v_{\mathrm{C} 9 \mathrm{C} 16}(7), \\
\omega_{\mathrm{C} 2 \mathrm{H} 22 \mathrm{H} 21}(6), \rho_{\mathrm{N} 3 \mathrm{H} 23}(5)\end{array}$ \\
\hline 69 & 1479.4 & 0.4 & 3.9 & $\begin{array}{l}\rho_{\mathrm{C} 15 \mathrm{H} 29}(17), \rho_{\mathrm{C} 13 \mathrm{H} 27}(15), v_{\mathrm{C} 10 \mathrm{C} 14}(8), v_{\mathrm{C} 11 \mathrm{C} 12}(7), \rho_{\mathrm{C} 19 \mathrm{H} 30}(6), \\
v_{\mathrm{C} 8 \mathrm{C} 16}(5)\end{array}$ \\
\hline 70 & 1489.3 & 14.6 & 1.3 & $\rho_{\mathrm{C} 19 \mathrm{H} 30}(12), v_{\mathrm{C} 8 \mathrm{C} 16}(10), \rho_{\mathrm{C} 6 \mathrm{H} 24}(8), \rho_{\mathrm{C} 13 \mathrm{H} 27}(6), v_{\mathrm{C} 7 \mathrm{C} 18}(6), v_{\mathrm{C} 8 \mathrm{C} 17}(5)$, \\
\hline
\end{tabular}




\begin{tabular}{|c|c|c|c|c|}
\hline & & & & $v_{\mathrm{N} 3 \mathrm{C} 18}(5)$ \\
\hline 71 & 1495.7 & 0.8 & 1.2 & $\delta_{1 / \mathrm{C}_{20} \mathrm{H} 31 \mathrm{H} 32}(88)$ \\
\hline 72 & 1507.8 & 6.5 & 2.0 & $\rho_{\mathrm{C} 14 \mathrm{H} 28}(20), \rho_{\mathrm{C} 12 \mathrm{H} 26}(20), v_{\mathrm{C} 10 \mathrm{C} 11}(17), v_{\mathrm{C} 13 \mathrm{C} 15}(8), \rho_{\mathrm{C} 13 \mathrm{H} 27}(5)$ \\
\hline 73 & 1527.1 & 64.7 & 1.0 & $\delta_{1 / \mathrm{C}_{2} \mathrm{H}_{22} \mathrm{H}_{21}}(86)$ \\
\hline 74 & 1550.0 & 227.6 & 1.1 & $\rho_{\mathrm{N} 3 \mathrm{H} 23}(19), v_{\mathrm{C} 6 \mathrm{C} 19}(18), v_{\mathrm{N} 3 \mathrm{C} 18}(15), \rho_{\mathrm{C} 6 \mathrm{H} 24}(11), v_{\mathrm{C} 6 \mathrm{C} 7}(6), v_{\mathrm{C} 8 \mathrm{C} 16}(6)$ \\
\hline 75 & 1610.6 & 80.7 & 13.6 & $\begin{array}{l}\rho_{\mathrm{N} 3 \mathrm{H} 23}(11), v_{\mathrm{C} 6 \mathrm{C} 19}(10), v_{\mathrm{C} 8 \mathrm{C} 19}(10), v_{\mathrm{C} 16 \mathrm{C} 18}(9),{ }^{1} \beta_{2 / R 6}(6), v_{\mathrm{C} 13 \mathrm{C} 15}(5), \\
v_{\mathrm{C} 10 \mathrm{C} 11}(5)\end{array}$ \\
\hline 76 & 1612.3 & 76.4 & 1.1 & $\begin{array}{l}\rho_{\mathrm{N} 3 \mathrm{H} 23}(13), v_{\mathrm{C} 13 \mathrm{C} 15}(13), v_{\mathrm{C} 10 \mathrm{C} 11}(13), v_{\mathrm{O} 5 \mathrm{C} 9}(8), v_{\mathrm{C} 12 \mathrm{C} 13}(6),{ }^{3} \beta_{3 / R 6}(6), \\
v_{\mathrm{C} 10 \mathrm{C} 14}(5)\end{array}$ \\
\hline 77 & 1626.0 & 45.8 & 18.8 & $v_{\mathrm{C} 14 \mathrm{C} 15}(11), v_{\mathrm{O} 5 \mathrm{C} 9}(10), v_{\mathrm{C} 11 \mathrm{C} 12}(9), v_{\mathrm{C} 6 \mathrm{C} 7}(9), v_{\mathrm{C} 8 \mathrm{C} 19}(6),{ }^{3} \beta_{2 / R 6}(6)$ \\
\hline 78 & 1630.9 & 263.0 & 6.0 & $v_{\mathrm{C} 6 \mathrm{C} 7}(12), v_{\mathrm{C} 14 \mathrm{C} 15}(9), v_{\mathrm{C}_{12} \mathrm{C} 13}(8), v_{\mathrm{C}_{11} \mathrm{C}_{12}}(7), v_{\mathrm{C} 10 \mathrm{C} 14}(6), v_{\mathrm{O} 4 \mathrm{C} 17}(6)$ \\
\hline 79 & 1669.1 & 231.1 & 27.8 & $v_{\mathrm{O} 5 \mathrm{C} 9}(41),{ }^{2} \beta_{3 / R 6}(6),{ }^{2} \beta_{\text {trig/R6 }}(6)$ \\
\hline 80 & 1721.0 & 179.1 & 29.4 & $v_{\mathrm{O} 4 \mathrm{C} 17}(71),{ }^{2} \beta_{\text {trig/R6}}(9)$ \\
\hline 81 & 2677.7 & 3.8 & 13.8 & $v_{\mathrm{S} 1 \mathrm{H} 33}(100)$ \\
\hline 82 & 2993.9 & 44.5 & 12.0 & $v_{\mathrm{C} 2 \mathrm{H} 22}(49), v_{\mathrm{C} 2 \mathrm{H} 21}(49)$ \\
\hline 83 & 3020.6 & 17.6 & 4.9 & $v_{\mathrm{C} 2 \mathrm{H} 21}(49), v_{\mathrm{C} 2 \mathrm{H} 22}(49)$ \\
\hline 84 & 3058.5 & 19.1 & 3.9 & $v_{\mathrm{C} 20 \mathrm{H} 31}(50), v_{\mathrm{C}_{20} \mathrm{H} 32}(50)$ \\
\hline 85 & 3112.4 & 7.2 & 1.6 & $v_{\mathrm{C}_{20 \mathrm{H} 32}}(49), v_{\mathrm{C} 20 \mathrm{H} 31}(49)$ \\
\hline 86 & 3169.9 & 3.4 & 4.4 & $v_{\mathrm{C} 15 \mathrm{H} 29}(49), v_{\mathrm{C} 13 \mathrm{H} 27}(45)$ \\
\hline 87 & 3173.1 & 11.5 & 7.7 & $v_{\mathrm{C} 6 \mathrm{H} 24}(90), v_{\mathrm{C} 7 \mathrm{H} 25}(6)$ \\
\hline 88 & 3184.7 & 13.5 & 14.4 & $v_{\mathrm{C} 13 \mathrm{H} 27}(43), v_{\mathrm{C} 15 \mathrm{H} 29}(42), v_{\mathrm{C} 12 \mathrm{H} 26}(9), v_{\mathrm{C} 14 \mathrm{H} 28}(5)$ \\
\hline 89 & 3201.4 & 3.7 & 6.7 & $v_{\mathrm{C} 12 \mathrm{H} 26}(81), v_{\mathrm{C} 14 \mathrm{H} 28}(9), v_{\mathrm{C} 13 \mathrm{H} 27}(9)$ \\
\hline 90 & 3205.6 & 10.4 & 11.0 & $v_{\mathrm{C} 14 \mathrm{H} 28}(82), v_{\mathrm{C} 15 \mathrm{H} 29}(8), v_{\mathrm{C}_{12} \mathrm{H} 26}(7)$ \\
\hline 91 & 3206.2 & 6.7 & 9.6 & $v_{\mathrm{C} 7 \mathrm{H} 25}(88), v_{\mathrm{C} 19 \mathrm{H} 30}(7)$ \\
\hline 92 & 3212.8 & 4.2 & 13.2 & $v_{\mathrm{C} 19 \mathrm{H} 30}(90)$ \\
\hline 93 & 3456.2 & 87.8 & 34.1 & $v_{\mathrm{N} 3 \mathrm{H} 23}(99)$ \\
\hline
\end{tabular}


Table S.14. Frequencies of normal vibrations, IR and Raman intensities and PEDs for TT-G conformer calculated at the B3LYP/6-311++G(d,p) level of theory.

\begin{tabular}{|c|c|c|c|c|}
\hline $\mathbf{N r}$ & $\begin{array}{l}\text { Freq. } \\
{\left[\mathrm{cm}^{-1}\right]}\end{array}$ & IR Int. & \begin{tabular}{c|} 
Raman \\
Int.
\end{tabular} & PED (contributions $>5 \%$ ) \\
\hline 1 & 25.6 & 0.2 & 11.3 & $\tau_{\mathrm{N} 3 \mathrm{C} 18}(41), \tau_{\mathrm{C} 2 \mathrm{~N} 3}(30),{ }^{1} \tau_{3 / R 6}(7), \omega_{\mathrm{N} 3 \mathrm{H} 23}(6)$ \\
\hline 2 & 39.3 & 4.6 & 0.3 & ${ }^{2} \tau_{3 / R 6}(44),{ }^{1} \tau_{3 / R 6}(14),{ }^{2} \tau_{2 / R 6}(12), \tau_{\mathrm{C} 2 \mathrm{~N} 3}(7)$ \\
\hline 3 & 57.1 & 2.3 & 0.8 & $\tau_{\mathrm{C} 2 \mathrm{~N} 3}(32), \omega_{\mathrm{N}_{3} \mathrm{H} 23}(30), \tau_{\mathrm{C}_{2} \mathrm{C} 20}(12),{ }^{2} \tau_{2 / R 6}(7),{ }^{2} \tau_{3 / R 6}(6)$ \\
\hline 4 & 60.1 & 0.1 & 1.3 & $\beta_{\mathrm{C}_{18 \mathrm{~N} 3} \mathrm{C}_{2}}(29), \delta_{2 / \mathrm{C}_{2} \mathrm{H} 22 \mathrm{H} 21}(27), \rho_{\mathrm{C} 18 \mathrm{~N} 3}(13), \delta_{2 / \mathrm{C}_{20} \mathrm{H}_{31} \mathrm{H}_{32}}(11)$ \\
\hline 5 & 83.0 & 0.6 & 0.1 & ${ }^{2} \tau_{2 / R 6}(29),{ }^{1} \tau_{3 / R \sigma}(12),{ }^{1} \tau_{2 / R 6}(10), \tau_{\mathrm{C}_{2 N} 3}(8),{ }^{2} \tau_{3 / R 6}(8)$ \\
\hline 6 & 119.2 & 0.1 & 0.7 & ${ }^{2} \tau_{1 / R 6}(39),{ }^{3} \tau_{2 / R 6}(17),{ }^{2} \tau_{2 / R 6}(15),{ }^{3} \tau_{3 / R 6}(8)$ \\
\hline 7 & 147.7 & 3.9 & 0.7 & $\omega_{\mathrm{N} 3 \mathrm{H} 23}(38), \tau_{\mathrm{C}_{2} \mathrm{C} 20}(30),{ }^{2} \tau_{1 / R 6}(8),{ }^{2} b f l_{R R}(6)$ \\
\hline 8 & 168.2 & 2.0 & 0.0 & ${ }^{4} b f l_{R R}(25),{ }^{2} b f l_{R R}(17), \tau_{N 3 C l 8}(15),{ }^{2} \tau_{3 / R 6}(8), \omega_{C 17 O 4}(6),{ }^{1} \tau_{3 / R 6}(5)$ \\
\hline 9 & 178.2 & 0.9 & 0.8 & $\delta_{2 / \mathrm{C} 20 \mathrm{H} 31 \mathrm{H} 32}(35), \rho_{\mathrm{C} 18 \mathrm{~N} 3_{3}}(8), v_{\mathrm{C}_{2} \mathrm{~N} 3}(8), \tau_{\mathrm{S} 1 \mathrm{C} 20}(8)$ \\
\hline 10 & 200.9 & 1.7 & 1.0 & ${ }^{1} \tau_{3 / R 6}(24),{ }^{\mathrm{l}} \tau_{2 / R \sigma}(22),{ }^{4} b f l_{R R}(15), \omega_{C 19 H 30}(6), \tau_{S I C 20}(6)$ \\
\hline 11 & 214.8 & 16.3 & 0.2 & $\tau_{\mathrm{S} 1 \mathrm{C} 20_{2}}(78)$ \\
\hline 12 & 228.1 & 0.9 & 1.0 & $\begin{array}{l}\delta_{2 / \mathrm{C} 2 \mathrm{H} 22 \mathrm{H} 21}(16),{ }^{1} \beta_{3 / R \sigma}(14),{ }^{2} \beta_{2 / R 6}(12), v_{\mathrm{C} 8 \mathrm{C} 17}(8), v_{\mathrm{S}_{1} \mathrm{C} 20}(6), \\
{ }^{1} \beta_{\text {trig/R6}}(5)\end{array}$ \\
\hline 13 & 268.8 & 0.7 & 0.6 & $\begin{array}{l}{ }^{2} b f l_{R R}(29), \omega_{N_{3} H_{23}}(10), \omega_{C l 8 N 3}(9),{ }^{1} \tau_{2 / R 6}(8), \omega_{C 7 H 25}(6),{ }^{l} \tau_{3 / R 6}(6), \\
{ }^{l} \tau_{1 / R 6}(6),{ }^{4} b f l_{R R}(5)\end{array}$ \\
\hline 14 & 295.6 & 0.2 & 0.6 & $\delta_{2 / \mathrm{C} 2 \mathrm{H} 22 \mathrm{H} 21}(19), \delta_{2 / \mathrm{C}_{20} \mathrm{H}_{31} \mathrm{H} 32}(17), \rho_{\mathrm{C} 18 \mathrm{~N} 3}(16), \beta_{\mathrm{C} 18 \mathrm{~N} 3 \mathrm{C} 2_{2}}(11)$ \\
\hline 15 & 342.5 & 1.4 & 0.2 & ${ }^{2} \beta_{2 / R 6}(24),{ }^{2} \beta_{3 / R 6}(21), \rho_{\mathrm{C} 17 \mathrm{O} 4}(21), \rho_{\mathrm{C} 18 \mathrm{~N} 3}(10), \delta_{2 / \mathrm{C} 20 \mathrm{H} 31 \mathrm{H} 32}(5)$ \\
\hline 16 & 356.3 & 2.6 & 0.4 & $\begin{array}{l}{ }^{2} \beta_{2 / R 6}(17),{ }^{2} \beta_{3 / R 6}(16), \delta_{2 / \mathrm{C} 20 \mathrm{H} 31 \mathrm{H} 32}(11), v_{\mathrm{C} 9 \mathrm{C} 10}(9), v_{\mathrm{C} 11 \mathrm{C} 17}(6), \\
v_{\mathrm{C} 8 \mathrm{C} 17}(6)\end{array}$ \\
\hline 17 & 405.7 & 8.9 & 0.5 & $\begin{array}{l}{ }^{2} \beta_{3 / R 6}(17),{ }^{2} \beta_{2 / R 6}(12),{ }^{1} \beta_{2 / R \sigma}(10), v_{\mathrm{C} 11 \mathrm{C} 17}(5),{ }^{3} \beta_{2 / R 6}(5), \\
\delta_{2 / \mathrm{C} 20 \mathrm{H} 31 \mathrm{H} 32}(5)\end{array}$ \\
\hline 18 & 426.4 & 17.8 & 0.5 & $\rho_{\mathrm{C} 9 \mathrm{O} 5}(29),{ }^{3} \tau_{3 / R 6}(20), \rho_{\mathrm{C} 17 \mathrm{O} 4}(11), v_{\mathrm{C} 9 \mathrm{C} 16}(7),{ }^{1} \beta_{3 / R 6}(6)$ \\
\hline 19 & 427.1 & 3.8 & 0.1 & ${ }^{3} \tau_{3 / R 6}(57),{ }^{4} b f l_{R R}(13)$ \\
\hline 20 & 433.5 & 0.1 & 0.0 & ${ }^{3} \tau_{2 / R \sigma}(33),{ }^{1} \tau_{2 / R \sigma}(20),{ }^{1} \tau_{3 / R \sigma}(17), \omega_{\mathrm{C} 9 \mathrm{O} 5}(6),{ }^{2} \tau_{1 / R \sigma}(6)$ \\
\hline 21 & 478.9 & 2.6 & 9.6 & $\begin{array}{l}{ }^{2} \beta_{2 / R 6}(29),{ }^{2} \beta_{3 / R 6}(20),{ }^{3} \beta_{3 / R \sigma}(10),{ }^{1} \beta_{3 / R 6}(7), v_{\mathrm{C} 8 \mathrm{C} 16}(6), v_{\mathrm{C} 11 \mathrm{C} 17}(5), \\
v_{\mathrm{C} 10 \mathrm{C} 11}(5)\end{array}$ \\
\hline 22 & 490.5 & 2.0 & 0.0 & ${ }^{3} \tau_{2 / R \sigma}(23),{ }^{1} \tau_{3 / R \sigma}(23),{ }^{1} \tau_{2 / R \sigma}(11),{ }^{2} \tau_{2 / R 6}(9),{ }^{1} \tau_{1 / R 6}(5),{ }^{3} \tau_{3 / R \sigma}(5)$ \\
\hline 23 & 498.6 & 5.1 & 3.2 & $\begin{array}{l}{ }^{1} \beta_{2 / R 6}(17), \rho_{\mathrm{C} 18 \mathrm{~N} 3}(16),{ }^{3} \beta_{2 / R \sigma}(10),{ }^{2} \beta_{3 / R 6}(8), \beta_{\mathrm{C} 18 \mathrm{~N} 3 \mathrm{C} 2}(7),{ }^{2} \beta_{2 / R 6}(6), \\
{ }^{1} \beta_{3 / R 6}(6)\end{array}$ \\
\hline 24 & 557.0 & 7.5 & 0.0 & $\omega_{\mathrm{C} 18 \mathrm{~N} 3}(29),{ }^{1} \tau_{3 / R 6}(14),{ }^{1} \tau_{2 / R \sigma}(14),{ }^{1} \tau_{1 / R 6}(10),{ }^{2} b f l_{R R}(6)$ \\
\hline 25 & 612.3 & 3.1 & 1.1 & ${ }^{1} \beta_{2 / R 6}(19),{ }^{3} \beta_{2 / R 6}(15),{ }_{3 / R \sigma}^{1}(15),{ }^{2} \beta_{\text {trig/R6 }}(12),{ }^{3} \beta_{3 / R \sigma}(6)$ \\
\hline 26 & 635.7 & 0.4 & 1.0 & ${ }^{2} \beta_{\text {trig/R6}}(36),{ }_{2 / R 6}(11),{ }_{1}^{1} \beta_{3 / R 6}(9),{ }^{3} \beta_{3 / R 6}(8), \rho_{\mathrm{C} 18 \mathrm{~N} 3}(7), \beta_{\mathrm{C} 18 \mathrm{~N} 3 \mathrm{C} 2}(7)$ \\
\hline 27 & 640.9 & 44.2 & 0.1 & $\tau_{\mathrm{N} 3 \mathrm{C} 18}(44), \omega_{\mathrm{N} 3 \mathrm{H} 23}(32), \tau_{\mathrm{C} 2 \mathrm{~N} 3}(5)$ \\
\hline 28 & 673.6 & 7.1 & 0.0 & ${ }^{3} \tau_{1 / R 6}(39),{ }^{1} \tau_{1 / R 6}(18), \omega_{\mathrm{C} 9 \mathrm{O} 5}(12), \omega_{\mathrm{C} 18 \mathrm{~N} 3}(11)$ \\
\hline 29 & 677.1 & 6.9 & 1.3 & ${ }^{3} \beta_{3 / R 6}(39),{ }^{3} \beta_{2 / R 6}(8), v_{\mathrm{S} 1 \mathrm{C} 20}(8), v_{\mathrm{C} 10 \mathrm{C} 11}(7)$ \\
\hline 30 & 705.2 & 0.3 & 0.4 & $\rho_{\mathrm{C} 9 \mathrm{O} 5}(19), \rho_{\mathrm{C} 17 \mathrm{O} 4}(17),{ }^{3} \beta_{2 / R 6}(16),{ }^{1} \beta_{2 / R 6}(9),{ }^{1} \beta_{3 / R 6}(9),{ }^{3} \beta_{3 / R 6}(6)$ \\
\hline 31 & 723.6 & 35.2 & 0.0 & ${ }^{3} \tau_{1 / R 6}(27),{ }^{1} \tau_{1 / R 6}(26), \omega_{\mathrm{C} 6 \mathrm{H} 24}(9), \omega_{\mathrm{C}_{15 \mathrm{H} 29}}(7), \omega_{\mathrm{C} 17 \mathrm{O} 4}(6)$ \\
\hline 32 & 732.4 & 9.0 & 0.5 & $\rho_{\mathrm{C} 20 \mathrm{H} 31 \mathrm{H} 32}(29), v_{\mathrm{S} 1 \mathrm{C} 20_{2}}(19), \beta_{\mathrm{C}_{20 \mathrm{~S} 1 \mathrm{H} 33}}(16)$ \\
\hline 33 & 746.7 & 53.5 & 0.0 & $\begin{array}{l}{ }^{1} \tau_{1 / R 6}(14), \omega_{\mathrm{C} 13 \mathrm{H} 27}(14), \omega_{\mathrm{C} 18 \mathrm{~N} 3}(13), \omega_{\mathrm{C} 9 \mathrm{O} 5}(9), \omega_{\mathrm{C} 17 \mathrm{O} 4}(8), \\
\omega_{\mathrm{C} 15 \mathrm{H} 29}(7), \omega_{\mathrm{C} 14 \mathrm{H} 28}(7),{ }^{3} \tau_{1 / R 6}(6)\end{array}$ \\
\hline 34 & 768.5 & 4.9 & 0.5 & $v_{\mathrm{S} 1 \mathrm{C} 20_{2}}(27), \rho_{\mathrm{C} 20 \mathrm{H} 31 \mathrm{H} 32}(25), \rho_{\mathrm{C} 2 \mathrm{H} 22 \mathrm{H} 21}(9)$ \\
\hline 35 & 785.7 & 8.2 & 0.1 & $\begin{array}{l}{ }^{3} \tau_{1 / R 6}(19),{ }^{2} \tau_{1 / R 6}(14), \omega_{\mathrm{C} 7 \mathrm{H} 25}(12), \omega_{\mathrm{C} 19 \mathrm{H} 30}(11), \omega_{\mathrm{C} 9 \mathrm{O} 5}(10), \\
\omega_{\mathrm{C} 17 \mathrm{O} 4}(10), \omega_{\mathrm{C} 6 \mathrm{H} 24}(10)\end{array}$ \\
\hline 36 & 802.5 & 2.2 & 0.9 & $\begin{array}{l}{ }^{1} \beta_{\text {trig/R6 }}(17),{ }^{3} \beta_{2 / R \sigma}(10),{ }^{3} \beta_{\text {trig/R6 }}(9),{ }^{2} \beta_{\text {trig/R6 }}(9),{ }^{1} \beta_{3 / R \sigma}(8), \\
{ }^{1} \beta_{2 / R \sigma}(8)\end{array}$ \\
\hline
\end{tabular}




\begin{tabular}{|c|c|c|c|c|}
\hline 37 & 810.0 & 1.0 & 0.1 & $\begin{array}{l}\omega_{\mathrm{C} 9 \mathrm{O} 5}(26), \omega_{\mathrm{C} 13 \mathrm{H} 27}(14),{ }^{1} \tau_{1 / R 6}(11), \omega_{\mathrm{C} 18 \mathrm{~N} 3}(9), \omega_{\mathrm{C} 15 \mathrm{H} 29}(8), \\
{ }^{2} \tau_{1 / R 6}(7)\end{array}$ \\
\hline 38 & 827.5 & 28.9 & 0.0 & $\begin{array}{l}\omega_{\mathrm{C} 7 \mathrm{H} 25}(22),{ }^{1} \tau_{1 / R 6}(18), \omega_{\mathrm{C}_{170} 4}(17), \omega_{\mathrm{C} 18 \mathrm{~N} 3}(10),{ }^{2} \tau_{1 / R \sigma}(8), \\
\omega_{\mathrm{C}_{15 \mathrm{H} 29}}(5), \omega_{\mathrm{C} 6 \mathrm{H} 24}(5)\end{array}$ \\
\hline 39 & 880.5 & 6.4 & 0.4 & $\beta_{\mathrm{C}_{20 \mathrm{~S} 1 \mathrm{H} 33}}(38), \rho_{\mathrm{C} 2 \mathrm{H} 22 \mathrm{H} 21}(19), \tau_{\mathrm{C} 20 \mathrm{H} 31 \mathrm{H} 32}(15)$ \\
\hline 40 & 898.0 & 33.1 & 3.0 & $\rho_{\mathrm{C} 17 \mathrm{O} 4}(10), v_{\mathrm{C} 7 \mathrm{C} 18}(9), \beta_{\mathrm{C}_{20 \mathrm{~S} 1 \mathrm{H} 33}}(8), v_{\mathrm{C} 16 \mathrm{C} 18}(8)$ \\
\hline 41 & 909.8 & 1.0 & 0.1 & $\omega_{\mathrm{C} 19 \mathrm{H} 30}(28), \omega_{\mathrm{C} 7 \mathrm{H} 25}(21), \omega_{\mathrm{C} 12 \mathrm{H} 26}(13), \omega_{\mathrm{C} 14 \mathrm{H} 28}(13), \omega_{\mathrm{C} 15 \mathrm{H} 29}(6)$ \\
\hline 42 & 916.4 & 0.8 & 0.0 & $\begin{array}{l}\omega_{\mathrm{C} 19 \mathrm{H} 30}(20), \omega_{\mathrm{C} 14 \mathrm{H} 28}(18), \omega_{\mathrm{C} 12 \mathrm{H} 26}(16), \omega_{\mathrm{C} 7 \mathrm{H} 25}(13), \omega_{\mathrm{C} 13 \mathrm{H} 27}(9), \\
\omega_{\mathrm{C} 15 \mathrm{H} 29}(6)\end{array}$ \\
\hline 43 & 929.4 & 3.0 & 1.3 & ${ }^{1} \beta_{\text {trig/R6 }}(25),{ }^{3} \beta_{\text {trig/R6}}(24), \rho_{\mathrm{C} 9 \mathrm{O} 5}(15), v_{\mathrm{C} 16 \mathrm{C} 18}(7)$ \\
\hline 44 & 998.9 & 0.0 & 0.0 & $\omega_{\mathrm{C} 6 \mathrm{H} 24}(47), \omega_{\mathrm{C} 19 \mathrm{H} 30}(20), \omega_{\mathrm{C} 7 \mathrm{H} 25}(9),{ }^{1} \tau_{1 / R 6}(7)$ \\
\hline 45 & 1001.6 & 1.9 & 0.0 & $\omega_{\mathrm{C} 12 \mathrm{H} 26}(29), \omega_{\mathrm{C} 14 \mathrm{H} 28}(28), \omega_{\mathrm{C} 15 \mathrm{H} 29}(10), \omega_{\mathrm{C} 13 \mathrm{H} 27}(9), \omega_{\mathrm{C} 6 \mathrm{H} 24}(8)$ \\
\hline 46 & 1012.3 & 88.6 & 3.3 & $v_{\mathrm{C} 8 \mathrm{C} 17}(15), v_{\mathrm{C} 2 \mathrm{~N}_{3}}(13), v_{\mathrm{C} 11 \mathrm{C} 12}(8), \rho_{\mathrm{C} 17 \mathrm{O} 4}(6), v_{\mathrm{C} 8 \mathrm{C} 19}(6), v_{\mathrm{C}_{13} \mathrm{C}_{15}}(6)$ \\
\hline 47 & 1015.8 & 0.0 & 0.0 & $\omega_{\mathrm{C} 15 \mathrm{H} 29}(30), \omega_{\mathrm{C} 13 \mathrm{H} 27}(29), \omega_{\mathrm{C} 14 \mathrm{H} 28}(14), \omega_{\mathrm{C} 12 \mathrm{H} 26}(14),{ }^{3} \tau_{1 / R 6}(12)$ \\
\hline 48 & 1056.3 & 2.4 & 1.6 & $v_{\mathrm{C}_{2} \mathrm{C} 20}(63), v_{\mathrm{C} 13 \mathrm{C} 15}(5)$ \\
\hline 49 & 1058.3 & 0.2 & 3.7 & $\begin{array}{l}v_{\mathrm{C} 13 \mathrm{C} 15}(26), v_{\mathrm{C} 2 \mathrm{C} 20}(13), v_{\mathrm{C} 14 \mathrm{C} 15}(10), \rho_{\mathrm{C} 12 \mathrm{H} 26}(10), \rho_{\mathrm{C} 14 \mathrm{H} 28}(9), \\
v_{\mathrm{C} 12 \mathrm{C} 13}(8)\end{array}$ \\
\hline 50 & 1095.1 & 28.8 & 1.1 & $\begin{array}{l}v_{\mathrm{C} 6 \mathrm{C} 19}(24),{ }^{1} \beta_{\text {trig/R6}}(13), \rho_{\mathrm{C} 19 \mathrm{H} 30}(12), v_{\mathrm{C} 2 \mathrm{~N} 3}(9), \rho_{\mathrm{C} 2 \mathrm{H} 22 \mathrm{H} 21}(6), \\
v_{\mathrm{C} 6 \mathrm{C} 7}(5)\end{array}$ \\
\hline 51 & 1097.3 & 4.5 & 0.7 & $\rho_{\mathrm{C} 2 \mathrm{H} 22 \mathrm{H} 21}(28), \rho_{\mathrm{C} 20 \mathrm{H} 31 \mathrm{H} 32}(18), \beta_{\mathrm{C}_{20 \mathrm{~S} 1 \mathrm{H} 33}}(17), v_{\mathrm{C} 6 \mathrm{C} 19}(8), \tau_{\mathrm{C} 20 \mathrm{H} 31 \mathrm{H} 32}(6)$ \\
\hline 52 & 1113.3 & 1.2 & 0.3 & $\begin{array}{l}\beta_{\text {trig/R6 }}(38), v_{\mathrm{C} 12 \mathrm{C} 13}(8), v_{\mathrm{C} 14 \mathrm{C} 15}(8), \rho_{\mathrm{C} 13 \mathrm{H} 27}(8), \rho_{\mathrm{C} 15 \mathrm{H} 29}(7), \\
v_{\mathrm{C} 9 \mathrm{C} 10}(6), v_{\mathrm{C} 11 \mathrm{C} 17}(5), \rho_{\mathrm{C} 14 \mathrm{H} 28}(5)\end{array}$ \\
\hline 53 & 1136.3 & 4.8 & 1.0 & $v_{\mathrm{C}_{2} \mathrm{~N} 3}(47), \rho_{\mathrm{C} 18 \mathrm{~N} 3}(7), v_{\mathrm{C} 8 \mathrm{C} 17}(5)$ \\
\hline 54 & 1176.6 & 3.9 & 5.1 & $\rho_{\mathrm{C} 13 \mathrm{H} 27}(19), \rho_{\mathrm{C} 12 \mathrm{H} 26}(14), v_{\mathrm{C} 9 \mathrm{C} 16}(8), v_{\mathrm{C} 9 \mathrm{C} 10}(7),{ }^{1} \beta_{\text {trig/R6}}(5)$ \\
\hline 55 & 1184.8 & 15.9 & 0.6 & $\begin{array}{l}\rho_{\mathrm{C} 15 \mathrm{H} 29}(30), v_{\mathrm{C} 14 \mathrm{C} 15}(10), \rho_{\mathrm{C} 14 \mathrm{H} 28}(9),{ }^{3} \beta_{\text {trig/R6 }}(7),{ }^{1} \beta_{\text {trig/R6 }}(6), \\
\rho_{\mathrm{C} 13 \mathrm{H} 27}(6), v_{\mathrm{C} 9 \mathrm{C} 16}(6)\end{array}$ \\
\hline 56 & 1197.0 & 35.9 & 8.4 & $\rho_{\mathrm{C} 6 \mathrm{H} 24}(31), \rho_{\mathrm{C} 7 \mathrm{H} 25}(15), v_{\mathrm{C} 6 \mathrm{C} 7}(8), \rho_{\mathrm{C} 13 \mathrm{H} 27}(6)$ \\
\hline 57 & 1215.5 & 2.2 & 5.4 & $\rho_{\mathrm{C} 19 \mathrm{H} 30}(26), v_{\mathrm{C} 8 \mathrm{C} 19}(9), \rho_{\mathrm{C} 7 \mathrm{H} 25}(9), v_{\mathrm{C}_{11} \mathrm{C} 17}(8), \rho_{\mathrm{C} 14 \mathrm{H} 28}(7)$ \\
\hline 58 & 1220.4 & 1.4 & 0.1 & $\tau_{\mathrm{C} 20 \mathrm{H} 31 \mathrm{H} 32}(43), \tau_{\mathrm{C} 2 \mathrm{H} 22 \mathrm{H} 21}(36), \rho_{\mathrm{C} 2 \mathrm{H} 22 \mathrm{H} 21}(13)$ \\
\hline 59 & 1257.9 & 58.5 & 4.5 & $\begin{array}{l}\rho_{\mathrm{C} 12 \mathrm{H} 26}(14), v_{\mathrm{C} 7 \mathrm{C} 18}(11), \rho_{\mathrm{C} 14 \mathrm{H} 28}(7), v_{\mathrm{C} 11 \mathrm{C} 12}(6), v_{\mathrm{C} 9 \mathrm{C} 10}(6) \\
{ }^{2} \beta_{\text {trig/R6 }}(6), \rho_{\mathrm{C} 7 \mathrm{H} 25}(6), \omega_{\mathrm{C}_{20} \mathrm{H} 31 \mathrm{H} 32}(5)\end{array}$ \\
\hline 60 & 1283.7 & 51.2 & 2.4 & $\begin{array}{l}\omega_{\mathrm{C} 20 \mathrm{H} 31 \mathrm{H} 32}(33), \omega_{\mathrm{C} 2 \mathrm{H} 22 \mathrm{H} 21}(8), \rho_{\mathrm{C} 14 \mathrm{H} 28}(8),{ }^{2} \beta_{\text {trig/R6 }}(7), \rho_{\mathrm{C} 19 \mathrm{H} 30}(6), \\
v_{\mathrm{C} 16 \mathrm{C} 18}(5)\end{array}$ \\
\hline 61 & 1292.5 & 655.7 & 6.7 & $\begin{array}{l}\omega_{\mathrm{C} 20 \mathrm{H} 31 \mathrm{H} 32}(22), v_{\mathrm{C} 9 \mathrm{C} 10}(10), v_{\mathrm{C} 9 \mathrm{C} 16}(8), v_{\mathrm{C} 8 \mathrm{C} 16}(6), v_{\mathrm{C}_{11} \mathrm{C}_{17}}(5), \\
v_{\mathrm{C} 8 \mathrm{C} 17}(5)\end{array}$ \\
\hline 62 & 1316.7 & 49.2 & 2 & $\tau_{\mathrm{C} 2 \mathrm{H} 22 \mathrm{H} 21}(37), \tau_{\mathrm{C} 20 \mathrm{H} 31 \mathrm{H} 32}(20), v_{\mathrm{C} 11 \mathrm{C} 17}(7), v_{\mathrm{C} 8 \mathrm{C} 17}(6)$ \\
\hline 63 & 1317.8 & 61.9 & 1.2 & $\begin{array}{l}v_{\mathrm{C}_{11 \mathrm{C} 17}}(14), \tau_{\mathrm{C} 2 \mathrm{H} 22 \mathrm{H} 21}(14), v_{\mathrm{C} 8 \mathrm{C} 17}(11), \tau_{\mathrm{C}_{20} \mathrm{H} 31 \mathrm{H} 32}(7), \rho_{\mathrm{C} 12 \mathrm{H} 26}(7), \\
\rho_{\mathrm{C} 17 \mathrm{O} 4}(6),{ }^{2} \beta_{2 / R 6}(5)\end{array}$ \\
\hline 64 & 1334.9 & 117.5 & 5.6 & $\begin{array}{l}v_{\mathrm{N}_{3} \mathrm{C} 18}(20), \rho_{\mathrm{N} 3 \mathrm{H} 23}(14), v_{\mathrm{C} 16 \mathrm{C} 18}(10), \omega_{\mathrm{C} 20 \mathrm{H} 31 \mathrm{H} 32}(8), \rho_{\mathrm{C} 6 \mathrm{H} 24}(8), \\
\omega_{\mathrm{C} 2 \mathrm{H} 22 \mathrm{H} 21}(6), v_{\mathrm{C} 9 \mathrm{C} 16}(6)\end{array}$ \\
\hline 65 & 1358.6 & 17.4 & 0.9 & $\begin{array}{l}v_{\mathrm{C} 10 \mathrm{C} 14}(15), v_{\mathrm{C} 10 \mathrm{C} 11}(15), v_{\mathrm{C}_{11} \mathrm{C} 12}(14), v_{\mathrm{C} 13 \mathrm{C} 15}(14), v_{\mathrm{C} 12 \mathrm{C} 13}(13) \\
v_{\mathrm{C} 14 \mathrm{C} 15}(13)\end{array}$ \\
\hline 66 & 1376.2 & 10.2 & 3.4 & $\begin{array}{l}v_{\mathrm{C} 16 \mathrm{C} 18}(12), v_{\mathrm{C} 8 \mathrm{C} 16}(11), v_{\mathrm{C} 8 \mathrm{C} 19}(10), v_{\mathrm{C} 9 \mathrm{C} 16}(9), v_{\mathrm{C} 6 \mathrm{C} 19}(7), v_{\mathrm{C} 9 \mathrm{C} 10}(6), \\
\rho_{\mathrm{C} 9 \mathrm{O} 5}(6), v_{\mathrm{C} 7 \mathrm{C} 18}(5)\end{array}$ \\
\hline 67 & 1404.3 & 6.8 & $1 .($ & $\omega_{\mathrm{C} 2 \mathrm{H} 22 \mathrm{H} 21}(56), \omega_{\mathrm{C} 20 \mathrm{H} 31 \mathrm{H} 32}(6), v_{\mathrm{C} 16 \mathrm{C} 18}(6), v_{\mathrm{C}_{2} \mathrm{C} 20}(5)$ \\
\hline 68 & 1434.1 & 57.5 & 0.2 & $\rho_{\mathrm{C} 7 \mathrm{H} 25}(23), v_{\mathrm{C} 6 \mathrm{C} 7}(11), v_{\mathrm{C} 8 \mathrm{C} 19}(11), v_{\mathrm{C} 8 \mathrm{C} 16}(7), v_{\mathrm{C} 9 \mathrm{C} 16}(7), \rho_{\mathrm{N}_{3} \mathrm{H}_{23}}(6)$ \\
\hline 69 & 1479.0 & 1.3 & 3.7 & $\begin{array}{l}\rho_{\mathrm{C} 15 \mathrm{H} 29}(14), \rho_{\mathrm{C} 13 \mathrm{H} 27}(13), v_{\mathrm{C} 10 \mathrm{C} 14}(7), \rho_{\mathrm{C} 19 \mathrm{H} 30}(7), \delta_{1 / \mathrm{C} 20 \mathrm{H} 31 \mathrm{H} 32}(6), \\
v_{\mathrm{C} 8 \mathrm{C} 16}(6), v_{\mathrm{C} 11 \mathrm{C} 12}(6)\end{array}$ \\
\hline 70 & 1481.4 & 3.2 & 1.7 & $\delta_{1 / \mathrm{C}_{20} \mathrm{H}_{31} \mathrm{H}_{32}}(84)$ \\
\hline
\end{tabular}




\begin{tabular}{|c|c|c|c|c|}
\hline 71 & 1489.1 & 13.8 & 1.1 & $\begin{array}{l}\rho_{\mathrm{C} 19 \mathrm{H} 30}(12), v_{\mathrm{C} 8 \mathrm{C} 16}(10), \rho_{\mathrm{C} 6 \mathrm{H} 24}(8), \rho_{\mathrm{C} 13 \mathrm{H} 27}(7), v_{\mathrm{C} 7 \mathrm{C} 18}(6), \rho_{\mathrm{C} 15 \mathrm{H} 29}(5), \\
v_{\mathrm{C} 8 \mathrm{C} 17}(5)\end{array}$ \\
\hline 72 & 1507.5 & 7.6 & 2.1 & $\rho_{\mathrm{C} 14 \mathrm{H} 28}(20), \rho_{\mathrm{C} 12 \mathrm{H} 26}(19), v_{\mathrm{C} 10 \mathrm{C} 11}(17), v_{\mathrm{C} 13 \mathrm{C} 15}(8)$ \\
\hline 73 & 1520.0 & 56.1 & 0.8 & $\delta_{1 / \mathrm{C}_{2} \mathrm{H}_{22} \mathrm{H}_{21}(88)}$ \\
\hline 74 & 1548.9 & 243.7 & 1.1 & $\rho_{\mathrm{N} 3 \mathrm{H} 23}(19), v_{\mathrm{C} 6 \mathrm{C} 19}(18), v_{\mathrm{N} 3 \mathrm{C} 18}(16), \rho_{\mathrm{C} 6 \mathrm{H} 24}(11), v_{\mathrm{C} 8 \mathrm{C} 16}(6), v_{\mathrm{C} 6 \mathrm{C} 7}(6)$ \\
\hline 75 & 1609.9 & 89.2 & 13.6 & $\rho_{\mathrm{N} 3 \mathrm{H} 23}(13), v_{\mathrm{C} 6 \mathrm{C} 19}(11), v_{\mathrm{C} 16 \mathrm{C} 18}(10), v_{\mathrm{C} 8 \mathrm{C} 19}(10),{ }^{1} \beta_{2 / R 6}(7), v_{\mathrm{C} 7 \mathrm{C} 18}(5)$ \\
\hline 76 & 1612.0 & 68.7 & 0.9 & $\begin{array}{l}v_{\mathrm{C} 13 \mathrm{C} 15}(15), v_{\mathrm{C} 10 \mathrm{C} 11}(14), \rho_{\mathrm{N} 3 \mathrm{H} 23}(11), v_{\mathrm{O} 5 \mathrm{C} 9}(9), v_{\mathrm{C} 12 \mathrm{C} 13}(7),{ }^{3} \beta_{3 / R 6}(6), \\
v_{\mathrm{C} 10 \mathrm{C} 14}(6), \rho_{\mathrm{C} 13 \mathrm{H} 27}(5)\end{array}$ \\
\hline 77 & 1625.7 & 54.9 & 18.9 & $v_{\mathrm{C} 6 \mathrm{C} 7}(10), v_{\mathrm{C} 14 \mathrm{C} 15}(10), v_{\mathrm{O} 5 \mathrm{C} 9}(10), v_{\mathrm{C}_{11} \mathrm{C}_{12}}(8), v_{\mathrm{C} 8 \mathrm{C} 19}(8),{ }^{3} \beta_{2 / R 6}(5)$ \\
\hline 78 & 1630.5 & 257.0 & 6.7 & $\begin{array}{l}v_{\mathrm{C} 14 \mathrm{C} 15}(11), v_{\mathrm{C} 6 \mathrm{C} 7}(11), v_{\mathrm{C} 12 \mathrm{C} 13}(9), v_{\mathrm{C} 11 \mathrm{C} 12}(8), v_{\mathrm{C} 10 \mathrm{C} 14}(6), v_{\mathrm{O} 4 \mathrm{C} 17}(6), \\
{ }^{3} \beta_{2 / R 6}(5)\end{array}$ \\
\hline 79 & 1668.6 & 226.4 & 28.5 & $v_{\mathrm{O} 5 \mathrm{C} 9}(41),{ }^{2} \beta_{3 / R 6}(6),{ }^{2} \beta_{\text {trig/R6 }}(6)$ \\
\hline 80 & 1720.8 & 178.8 & 30.5 & $v_{\mathrm{O} 4 \mathrm{C} 17}(71),{ }^{2} \beta_{\text {trig/R6}}(9)$ \\
\hline 81 & 2659.2 & 5.1 & 8.6 & $v_{\mathrm{S} 1 \mathrm{H} 33}(100)$ \\
\hline 82 & 2991.7 & 55.4 & 11.0 & $v_{\mathrm{C} 2 \mathrm{H} 21}(83), v_{\mathrm{C}_{2} \mathrm{H} 22}(16)$ \\
\hline 83 & 3029.0 & 17.4 & 5.7 & $v_{\mathrm{C} 2 \mathrm{H} 22}(81), v_{\mathrm{C}_{2} \mathrm{H} 21}(16)$ \\
\hline 84 & 3061.3 & 12.7 & 3.9 & $v_{\mathrm{C}_{20 \mathrm{H} 32}}(50), v_{\mathrm{C} 20 \mathrm{H} 31}(49)$ \\
\hline 85 & 3110.7 & 7.6 & 1.6 & $v_{\mathrm{C}_{20} \mathrm{H} 31}(50), v_{\mathrm{C}_{20} \mathrm{H} 32}(48)$ \\
\hline 86 & 3169.9 & 3.4 & 4.5 & $v_{\mathrm{C} 15 \mathrm{H} 29}(51), v_{\mathrm{C} 13 \mathrm{H} 27}(43)$ \\
\hline 87 & 3172.1 & 11.5 & 7.7 & $v_{\mathrm{C} 6 \mathrm{H} 24}(90), v_{\mathrm{C} 7 \mathrm{H} 25}(6)$ \\
\hline 88 & 3184.8 & 13.6 & 14.6 & $v_{\mathrm{C} 13 \mathrm{H} 27}(45), v_{\mathrm{C} 15 \mathrm{H} 29}(40), v_{\mathrm{C}_{12} \mathrm{H} 26}(9)$ \\
\hline 89 & 3202.0 & 3.6 & 6.7 & $v_{\mathrm{C}_{12} \mathrm{H} 26}(81), v_{\mathrm{C} 14 \mathrm{H} 28}(9), v_{\mathrm{C} 13 \mathrm{H} 27}(9)$ \\
\hline 90 & 3204.7 & 8.4 & 8.1 & $v_{\mathrm{C} 7 \mathrm{H} 25}(90)$ \\
\hline 91 & 3206.1 & 8.9 & 12.6 & $v_{\mathrm{C} 14 \mathrm{H} 28}(83), v_{\mathrm{C} 15 \mathrm{H} 29}(7), v_{\mathrm{C} 12 \mathrm{H} 26}(7)$ \\
\hline 92 & 3212.5 & 3.7 & 12.8 & $v_{\mathrm{C} 19 \mathrm{H} 30}(92)$ \\
\hline 93 & 3452.1 & 87.9 & 33.9 & $v_{\mathrm{N} 3 \mathrm{H} 23}(99)$ \\
\hline
\end{tabular}


Table S.15. Frequencies of normal vibrations, IR and Raman intensities and PEDs for TGT conformer calculated at the B3LYP/6-311++G(d,p) level of theory.

\begin{tabular}{|c|c|c|c|c|}
\hline $\mathbf{N r}$ & $\begin{array}{l}\text { Freq. } \\
{\left[\mathrm{cm}^{-1}\right]}\end{array}$ & IR Int. & $\begin{array}{c}\text { Raman } \\
\text { Int. }\end{array}$ & PED $($ contributions $>5 \%)$ \\
\hline 1 & 20.6 & 0.5 & 13.5 & $\tau_{\mathrm{C} 2 \mathrm{~N} 3}(50),{ }^{1} \tau_{3 / R 6}(10), \omega_{\mathrm{N} 3 \mathrm{H} 23}(10), \tau_{\mathrm{S}_{1 \mathrm{C} 20}}(10)$ \\
\hline 2 & 34.6 & 4.1 & 5.0 & $\tau_{\mathrm{N} 3 \mathrm{C} 18}(29),{ }^{2} \tau_{3 / R 6}(22),{ }^{2} \tau_{2 / R 6}(17), \omega_{\mathrm{N} 3 \mathrm{H} 23}(11)$ \\
\hline 3 & 46.1 & 1.8 & 1.2 & ${ }^{2} \tau_{3 / R \sigma}(38), \omega_{\mathrm{N} 3 \mathrm{H} 23}(15),{ }^{2} \tau_{2 / R 6}(11),{ }^{1} \tau_{3 / R 6}(7), \tau_{\mathrm{C}_{2} \mathrm{~N} 3}(5)$ \\
\hline 4 & 71.7 & 0.2 & 0.2 & $\begin{array}{l}{ }^{2} \tau_{2 / R 6}(14), \tau_{\mathrm{C}_{2} \mathrm{C}_{20}}(10),{ }^{1} \tau_{3 / R 6}(9), \tau_{\mathrm{N} 3 \mathrm{C} 18}(8), \tau_{\mathrm{C} 2 \mathrm{~N} 3}(8),{ }^{1} \tau_{2 / R 6}(7), \\
\delta_{2 / \mathrm{C} 2 \mathrm{H} 22 \mathrm{H} 21}(6),{ }^{2} \tau_{3 / R 6}(6)\end{array}$ \\
\hline 5 & 102.2 & 2.2 & 0.4 & $\omega_{\mathrm{N} 3 \mathrm{H} 23}(30),{ }^{2} \tau_{2 / R 6}(19), \beta_{\mathrm{C} 18 \mathrm{~N} 3 \mathrm{C} 2_{2}}(12), \rho_{\mathrm{C} 18 \mathrm{~N} 3}(8), \delta_{2 / \mathrm{C} 2 \mathrm{H} 22 \mathrm{H} 21}(6)$ \\
\hline 6 & 116.1 & 8.1 & 0.6 & $\tau_{\mathrm{S}_{1 \mathrm{C} 20}}(35), \tau_{\mathrm{C}_{2} \mathrm{C} 20}(21), \omega_{\mathrm{N} 3 \mathrm{H} 23}(11),{ }^{2} \tau_{2 / R 6}(8)$ \\
\hline 7 & 120.5 & 2.4 & 1.6 & ${ }^{2} \tau_{1 / R 6}(32), \tau_{\mathrm{S} 1 \mathrm{C} 20}(18), \omega_{\mathrm{N} 3 \mathrm{H} 23}(17),{ }^{3} \tau_{2 / R 6}(9)$ \\
\hline 8 & 148.2 & 3.4 & 0.3 & 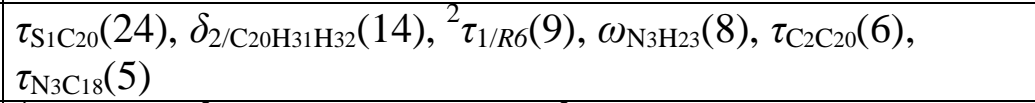 \\
\hline 9 & 165.5 & 0.3 & 0.0 & $\begin{array}{l}{ }^{4} b f l_{R R}(24),{ }^{2} b f l_{R R}(22), \tau_{N 3 C l 8}(10),{ }^{2} \tau_{3 / R 6}(8), \omega_{C 17 O 4}(6), \omega_{C 9 O 5}(6), \\
{ }^{l} \tau_{3 / R 6}(5)\end{array}$ \\
\hline 10 & 201.6 & 0.0 & 1.2 & ${ }^{1}{ }^{1} \tau_{3 / R 6}(26),{ }^{1} \tau_{2 / R 6}(22),{ }^{4} b f l_{R R}(17), \omega_{C 19 H 30}(7)$ \\
\hline 11 & 238.5 & 0.9 & 0.2 & $\begin{array}{l}\delta_{2 / \mathrm{C} 2 \mathrm{H} 22 \mathrm{H} 21}(13),{ }^{3} \beta_{2 / R 6}(9), \beta_{\mathrm{C} 18 \mathrm{~N} 3 \mathrm{C} 2}(9), \tau_{\mathrm{C}_{2} \mathrm{C} 20}(9),{ }^{1} \beta_{3 / R 6}(5), \\
v_{\mathrm{C} 9 \mathrm{C} 16}(5), v_{\mathrm{C} 8 \mathrm{C} 17}(5)\end{array}$ \\
\hline 12 & 251.9 & 1.4 & 0.8 & $\delta_{2 / \mathrm{C} 20 \mathrm{H} 31 \mathrm{H} 32}(26), \tau_{\mathrm{C}_{2} \mathrm{C} 20}(13), \beta_{\mathrm{C} 18 \mathrm{~N} 3 \mathrm{C} 2_{2}}(8), \delta_{2 / \mathrm{C}_{2} \mathrm{H} 22 \mathrm{H} 21}(7), \tau_{\mathrm{C}_{2} \mathrm{~N} 3}(5)$ \\
\hline 13 & 270.7 & 0.9 & 0.5 & $\begin{array}{l}{ }^{2} b f l_{R R}(27), \omega_{N 3 H 23}(11), \omega_{C I 8 N 3}(8),{ }^{I} \tau_{2 / R 6}(8), \omega_{C 7 H 25}(6),{ }^{I} \tau_{3 / R 6}(5), \\
{ }^{l} \tau_{1 / R 6}(5)\end{array}$ \\
\hline 14 & 309.6 & 5.0 & 0.2 & ${ }^{2} \beta_{2 / R 6}(37), \delta_{2 / \mathrm{C}_{20} \mathrm{H} 31 \mathrm{H} 32}(10), \beta_{\mathrm{C} 18 \mathrm{~N} 3 \mathrm{C} 2_{2}}(8), v_{\mathrm{C} 8 \mathrm{C} 17}(6), v_{\mathrm{C} 16 \mathrm{C} 18}(5)$ \\
\hline 15 & 342.4 & 1.6 & 0.3 & ${ }^{2} \beta_{3 / R 6}(26), \rho_{\mathrm{C} 17 \mathrm{O} 4}(21), v_{\mathrm{C} 8 \mathrm{C} 17}(7), \rho_{\mathrm{C} 18 \mathrm{~N} 3}(6)$ \\
\hline 16 & 365.7 & 5.8 & 1.3 & ${ }^{2} \beta_{3 / R 6}(19),{ }^{1} \beta_{3 / R 6}(14), \delta_{2 / \mathrm{C} 20 \mathrm{H} 31 \mathrm{H} 32}(14), v_{\mathrm{C} 11 \mathrm{C} 17}(11), v_{\mathrm{C} 9 \mathrm{C} 16}(6)$ \\
\hline 17 & 420.7 & 16.9 & 0.1 & $\begin{array}{l}\delta_{2 / \mathrm{C} 2 \mathrm{H} 22 \mathrm{H} 21}(20), \delta_{2 / \mathrm{C}_{20} \mathrm{H} 31 \mathrm{H} 32}(13),{ }^{2} \beta_{3 / R 6}(12), \rho_{\mathrm{C} 18 \mathrm{~N} 3}(10), \rho_{\mathrm{C} 17 \mathrm{O} 4}(8), \\
{ }^{1} \beta_{2 / R 6}(6)\end{array}$ \\
\hline 18 & 425.7 & 10.1 & 0.8 & $\rho_{\mathrm{C} 9 \mathrm{O} 5}(32), v_{\mathrm{C} 9 \mathrm{C} 16}(10), \rho_{\mathrm{C} 18 \mathrm{~N} 3}(9), \rho_{\mathrm{C}_{17} \mathrm{O} 4}(8), \delta_{2 / \mathrm{C} 2 \mathrm{H} 22 \mathrm{H} 21}(7)$ \\
\hline 19 & 427.4 & 1.2 & 0.1 & ${ }^{3} \tau_{3 / R \sigma}(61),{ }^{4} b f l_{R R}(14),{ }^{3} \tau_{2 / R \sigma}(5)$ \\
\hline 20 & 433.4 & 0.1 & 0.1 & ${ }^{3} \tau_{2 / R 6}(33),{ }^{1} \tau_{2 / R 6}(20),{ }^{1} \tau_{3 / R 6}(16), \omega_{\mathrm{C} 9 \mathrm{O} 5}(6),{ }^{2} \tau_{1 / R 6}(6)$ \\
\hline 21 & 472.0 & 3.1 & 1.3 & ${ }^{2} \beta_{2 / R 6}(34), \rho_{\mathrm{C} 18 \mathrm{~N} 3}(12),{ }^{1} \beta_{2 / R 6}(11),{ }^{3} \beta_{2 / R 6}(7),{ }^{3} \beta_{3 / R 6}(6)$ \\
\hline 22 & 485.4 & 0.3 & 9.7 & ${ }^{2} \beta_{3 / R 6}(28),{ }^{1} \beta_{3 / R \sigma}(9),{ }^{1} \beta_{2 / R 6}(7),{ }^{3} \beta_{2 / R 6}(6), \rho_{\mathrm{C} 18 \mathrm{~N} 3}(5),{ }^{2} \beta_{2 / R 6}(5)$ \\
\hline 23 & 491.4 & 2.3 & 0.1 & ${ }^{3} \tau_{2 / R \sigma}(23),{ }^{1} \tau_{3 / R \sigma}(23),{ }^{1} \tau_{2 / R \sigma}(11),{ }^{2} \tau_{2 / R \sigma}(9),{ }^{3} \tau_{3 / R \sigma}(5),{ }^{1} \tau_{1 / R \sigma}(5)$ \\
\hline 24 & 558.0 & 7.6 & 0.0 & $\omega_{\mathrm{C} 18 \mathrm{~N} 3}(29),{ }^{1} \tau_{3 / R 6}(14),{ }^{1} \tau_{2 / R \sigma}(13),{ }^{1} \tau_{1 / R 6}(11),{ }^{2} b f l_{R R}(6)$ \\
\hline 25 & 618.1 & 4.1 & 0.5 & 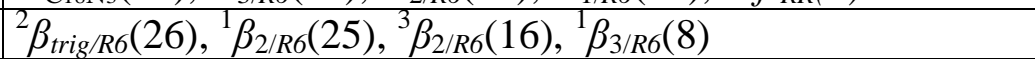 \\
\hline 26 & 639.7 & 17.9 & 0.8 & $\begin{array}{l}\tau_{\mathrm{N} 3 \mathrm{C} 18}(15),{ }^{2} \beta_{\text {trig/R6 }}(13),{ }^{3} \beta_{3 / R 6}(12),{ }^{\mathrm{i}} \beta_{3 / R 6}(11), \omega_{\mathrm{N} 3 \mathrm{H} 23}(10), \\
v_{\mathrm{S} 1 \mathrm{C} 20}(6)\end{array}$ \\
\hline 27 & 642.3 & 26.9 & 0.6 & $\tau_{\mathrm{N} 3 \mathrm{C} 18}(22), \omega_{\mathrm{N} 3 \mathrm{H} 23}(17),{ }^{2} \beta_{\text {trig/R6 }}(14),{ }^{3} \beta_{3 / R 6}(8),{ }^{1} \beta_{3 / R 6}(6)$ \\
\hline 28 & 663.8 & 3.5 & 1.4 & $v_{\mathrm{S}_{1} \mathrm{C} 20}(68), \delta_{2 / \mathrm{C} 20 \mathrm{H} 31 \mathrm{H} 32}(6)$ \\
\hline 29 & 673.6 & 9.4 & 0.1 & ${ }^{3} \tau_{1 / R 6}(38),{ }^{1} \tau_{1 / R 6}(18), \omega_{\mathrm{C} 9 \mathrm{O} 5}(12), \omega_{\mathrm{C} 18 \mathrm{~N} 3}(10)$ \\
\hline 30 & 686.4 & 7.7 & 2.2 & ${ }^{3} \beta_{3 / R 6}(32),{ }^{1} \beta_{3 / R 6}(13), \beta_{\mathrm{C} 18 \mathrm{~N} 3 \mathrm{C} 2}(6), v_{\mathrm{C} 10 \mathrm{C} 11}(5)$ \\
\hline 31 & 709.7 & 3.3 & 0.4 & ${ }^{3} \beta_{2 / R 6}(24), \rho_{\mathrm{C} 17 \mathrm{O} 4}(17), \rho_{\mathrm{C} 9 \mathrm{O} 5}(16),{ }^{1} \beta_{2 / R 6}(12), \rho_{\mathrm{C} 18 \mathrm{~N}_{3}}(5)$ \\
\hline 32 & 723.9 & 34.5 & 0.0 & ${ }^{3} \tau_{1 / R 6}(29),{ }^{1} \tau_{1 / R 6}(27), \omega_{\mathrm{C} 6 \mathrm{H} 24}(9), \omega_{\mathrm{C} 15 \mathrm{H} 29}(7), \omega_{\mathrm{C} 17 \mathrm{O} 4}(6)$ \\
\hline 33 & 746.5 & 54.2 & 0.0 & $\begin{array}{l}\omega_{\mathrm{C} 13 \mathrm{H} 27}(14),{ }^{1} \tau_{1 / R 6}(13), \omega_{\mathrm{C} 18 \mathrm{~N} 3}(13), \omega_{\mathrm{C} 9 \mathrm{O} 5}(9), \omega_{\mathrm{C} 17 \mathrm{O} 4}(9), \\
\omega_{\mathrm{C} 15 \mathrm{H} 29}(7), \omega_{\mathrm{C} 14 \mathrm{H} 28}(7),{ }^{3} \tau_{1 / R 6}(6)\end{array}$ \\
\hline 34 & 784.7 & 9.2 & 0.0 & $\begin{array}{l}{ }^{3} \tau_{1 / R 6}(19), \omega_{\mathrm{C} 7 \mathrm{H} 25}(14),{ }^{2} \tau_{1 / R 6}(13), \omega_{\mathrm{C} 19 \mathrm{H} 30}(11), \omega_{\mathrm{C} 9 \mathrm{O} 5}(10), \\
\omega_{\mathrm{C} 6 \mathrm{H} 24}(10), \omega_{\mathrm{C} 17 \mathrm{O} 4}(9)\end{array}$ \\
\hline 35 & 796.5 & 1.8 & 0.9 & $\begin{array}{l}{ }^{1} \beta_{\text {trig/R6 }}(18),{ }^{1} \beta_{3 / R 6}(13),{ }^{3} \beta_{2 / R \sigma}(11),{ }^{3} \beta_{\text {trig/R6 }}(8),{ }^{2} \beta_{\text {trig/R6 }}(7), \\
v_{\mathrm{C} 9 \mathrm{C} 10}(6)\end{array}$ \\
\hline
\end{tabular}




\begin{tabular}{|c|c|c|c|c|}
\hline 36 & 810.6 & 1.3 & 0.1 & $\begin{array}{l}\omega_{\mathrm{C} 9 \mathrm{O} 5}(26), \omega_{\mathrm{C} 13 \mathrm{H} 27}(14),{ }^{1} \tau_{1 / R 6}(11), \omega_{\mathrm{C} 18 \mathrm{~N} 3}(9), \omega_{\mathrm{C} 15 \mathrm{H} 29}(8), \\
{ }^{2} \tau_{1 / R 6}(6)\end{array}$ \\
\hline 37 & 825.7 & 25.6 & 0.1 & $\begin{array}{l}\omega_{\mathrm{C} 7 \mathrm{H} 25}(19),{ }^{1} \tau_{1 / R 6}(17), \omega_{\mathrm{C} 17 \mathrm{O} 4}(16), \omega_{\mathrm{C} 18 \mathrm{~N} 3}(9),{ }^{2} \tau_{1 / R \sigma}(7), \\
\omega_{\mathrm{C} 15 \mathrm{H} 29}(5)\end{array}$ \\
\hline 38 & 833.7 & 5.6 & 0.6 & $\rho_{\mathrm{C} 20 \mathrm{H} 31 \mathrm{H} 32}(31), \beta_{\mathrm{C}_{20 \mathrm{~S} 1 \mathrm{H} 33}}(13), \rho_{\mathrm{C} 2 \mathrm{H} 22 \mathrm{H} 21}(7), v_{\mathrm{C}_{2} \mathrm{C} 20}(5)$ \\
\hline 39 & 880.2 & 13.8 & 1.6 & $\beta_{\mathrm{C}_{20 \mathrm{~S} 1 \mathrm{H} 33}}(33), v_{\mathrm{C} 16 \mathrm{C} 18}(6), \rho_{\mathrm{C} 17 \mathrm{O} 4}(6), v_{\mathrm{C} 7 \mathrm{C} 18}(6)$ \\
\hline 40 & 908.5 & 0.9 & 0.0 & $\omega_{\mathrm{C} 19 \mathrm{H} 30}(39), \omega_{\mathrm{C} 7 \mathrm{H} 25}(27), \omega_{\mathrm{C} 12 \mathrm{H} 26}(6), \omega_{\mathrm{C} 14 \mathrm{H} 28}(6)$ \\
\hline 41 & 916.5 & 0.4 & 0.0 & $\begin{array}{l}\omega_{\mathrm{C} 14 \mathrm{H} 28}(25), \omega_{\mathrm{C} 12 \mathrm{H} 26}(23), \omega_{\mathrm{C} 13 \mathrm{H} 27}(12), \omega_{\mathrm{C} 15 \mathrm{H} 29}(10), \omega_{\mathrm{C} 19 \mathrm{H} 30}(9), \\
\omega_{\mathrm{C} 7 \mathrm{H} 25}(6)\end{array}$ \\
\hline 42 & 928.9 & 2.1 & 1.2 & ${ }^{1} \beta_{\text {trig/R6}}(25),{ }^{3} \beta_{\text {trig/R6 }}(24), \rho_{\mathrm{C} 9 \mathrm{O} 5}(14), v_{\mathrm{C} 16 \mathrm{C} 18}(7), \rho_{\mathrm{C} 17 \mathrm{O} 4}(6)$ \\
\hline 43 & 929.8 & 25.0 & 0.6 & $\beta_{\mathrm{C}_{20 \mathrm{~S} 1 \mathrm{H} 33}}(26), \rho_{\mathrm{C} 20 \mathrm{H} 31 \mathrm{H} 32}(20), \rho_{\mathrm{C} 17 \mathrm{O} 4}(6)$ \\
\hline 44 & 996.6 & 4.8 & 0.1 & $v_{\mathrm{C}_{2} \mathrm{C} 20}(21), \omega_{\mathrm{C} 6 \mathrm{H} 24}(19), \rho_{\mathrm{C} 2 \mathrm{H} 22 \mathrm{H} 21}(19), \omega_{\mathrm{C} 19 \mathrm{H} 30}(9), \delta_{2 / \mathrm{C} 2 \mathrm{H} 22 \mathrm{H} 21}(6)$ \\
\hline 45 & 999.6 & 2.4 & 0.1 & $\begin{array}{l}\omega_{\mathrm{C} 6 \mathrm{H} 24}(39), \omega_{\mathrm{C} 19 \mathrm{H} 30}(17), \omega_{\mathrm{C} 7 \mathrm{H} 25}(8), v_{\mathrm{C} 2 \mathrm{C} 20}(6),{ }^{1} \tau_{1 / R 6}(6), \\
\rho_{\mathrm{C}_{2} \mathrm{H} 22 \mathrm{H} 21}(5)\end{array}$ \\
\hline 46 & 1002.9 & 2.0 & 0.0 & $\omega_{\mathrm{C} 12 \mathrm{H} 26}(34), \omega_{\mathrm{C} 14 \mathrm{H} 28}(27), \omega_{\mathrm{C} 13 \mathrm{H} 27}(13), \omega_{\mathrm{C} 15 \mathrm{H} 29}(8)$ \\
\hline 47 & 1016.8 & 0.0 & 0.0 & $\omega_{\mathrm{C} 15 \mathrm{H} 29}(32), \omega_{\mathrm{C} 13 \mathrm{H} 27}(26), \omega_{\mathrm{C} 14 \mathrm{H} 28}(18),{ }^{3} \tau_{1 / R 6}(12), \omega_{\mathrm{C} 12 \mathrm{H} 26}(11)$ \\
\hline 48 & 1017.6 & 76.8 & 2.8 & $\begin{array}{l}v_{\mathrm{C} 8 \mathrm{C} 17}(12), \rho_{\mathrm{C} 20 \mathrm{H} 31 \mathrm{H} 32}(8), v_{\mathrm{C} 2 \mathrm{~N} 3}(6), \rho_{\mathrm{C} 2 \mathrm{H} 22 \mathrm{H} 21}(6), v_{\mathrm{C} 11 \mathrm{C} 12}(6), \\
v_{\mathrm{C}_{13} \mathrm{C} 15}(5), \tau_{\mathrm{C} 20 \mathrm{H} 31 \mathrm{H} 32}(5)\end{array}$ \\
\hline 49 & 1056.1 & 0.2 & 4.9 & $\begin{array}{l}v_{\mathrm{C} 13 \mathrm{C} 15}(30), v_{\mathrm{C} 14 \mathrm{C} 15}(11), v_{\mathrm{C} 12 \mathrm{C} 13}(10), \rho_{\mathrm{C} 12 \mathrm{H} 26}(10), \rho_{\mathrm{C} 14 \mathrm{H} 28}(9), \\
\tau_{\mathrm{C} 20 \mathrm{H} 31 \mathrm{H} 32}(6)\end{array}$ \\
\hline 50 & 1069.8 & 1.8 & 0.4 & $\begin{array}{l}\rho_{\mathrm{C} 2 \mathrm{H} 22 \mathrm{H} 21}(16), \tau_{\mathrm{C}_{2} 0 \mathrm{H} 31 \mathrm{H} 32}(14), v_{\mathrm{C}_{2} \mathrm{C} 20}(12), \beta_{\mathrm{C}_{20 \mathrm{~S} 1 \mathrm{H} 33}}(7),{ }^{1} \beta_{\text {trig/R6 }}(5), \\
\rho_{\mathrm{C} 20 \mathrm{H} 31 \mathrm{H} 32}(5)\end{array}$ \\
\hline 51 & 1098.7 & 17.0 & 2.0 & $v_{\mathrm{C} 6 \mathrm{C} 19}(30), \rho_{\mathrm{C} 19 \mathrm{H} 30}(16),{ }^{1} \beta_{\text {trig/R6 }}(11), v_{\mathrm{C} 6 \mathrm{C} 7}(10), \rho_{\mathrm{C} 7 \mathrm{H} 25}(6)$ \\
\hline 52 & 1113.0 & 1.1 & 0.3 & $\begin{array}{l}{ }^{3} \beta_{\text {trig/R6 }}(38), v_{\mathrm{C} 12 \mathrm{C} 13}(8), v_{\mathrm{C} 14 \mathrm{C} 15}(8), \rho_{\mathrm{C} 13 \mathrm{H} 27}(8), \rho_{\mathrm{C} 15 \mathrm{H} 29}(7), \\
v_{\mathrm{C} 9 \mathrm{C} 10}(6), v_{\mathrm{C} 11 \mathrm{C} 17}(5), \rho_{\mathrm{C} 14 \mathrm{H} 28}(5), \rho_{\mathrm{C} 12 \mathrm{H} 26}(5)\end{array}$ \\
\hline 53 & 1137.6 & 3.1 & 0.9 & $v_{\mathrm{C}_{2} \mathrm{~N} 3}(34), v_{\mathrm{C}_{2} \mathrm{C} 20}(17), \tau_{\mathrm{C} 20 \mathrm{H} 31 \mathrm{H} 32}(9), \rho_{\mathrm{C} 2 \mathrm{H} 22 \mathrm{H} 21}(8), \rho_{\mathrm{C} 18 \mathrm{~N} 3}(5)$ \\
\hline 54 & 1176.0 & 4.5 & 4.7 & $\begin{array}{l}\rho_{\mathrm{C} 13 \mathrm{H} 27}(18), \rho_{\mathrm{C} 12 \mathrm{H} 26}(13), v_{\mathrm{C} 9 \mathrm{C} 16}(9), v_{\mathrm{C} 9 \mathrm{C} 10}(7),{ }^{1} \beta_{\text {trig/R6 }}(6), \\
{ }^{3} \beta_{\text {trig/R6 }}(5)\end{array}$ \\
\hline 55 & 1183.5 & 19.2 & 0.4 & $\begin{array}{l}\rho_{\mathrm{C} 15 \mathrm{H} 29}(30), \rho_{\mathrm{C} 14 \mathrm{H} 28}(10), v_{\mathrm{C} 14 \mathrm{C} 15}(9),{ }^{1} \beta_{\text {trig/R6}}(7), \rho_{\mathrm{C} 13 \mathrm{H} 27}(6), \\
{ }^{3} \beta_{\text {trig/R6 }}(6)\end{array}$ \\
\hline 56 & 1197.2 & 29.6 & 8 & $\rho_{\mathrm{C} 6 \mathrm{H} 24}(29), \rho_{\mathrm{C} 7 \mathrm{H} 25}(13), \rho_{\mathrm{C} 13 \mathrm{H} 27}(7), v_{\mathrm{C} 6 \mathrm{C} 7}(7)$ \\
\hline 57 & 1210.7 & 8.3 & 4.4 & $\rho_{\mathrm{C} 19 \mathrm{H} 30}(17), \tau_{\mathrm{C} 20 \mathrm{H} 31 \mathrm{H} 32}(15), \rho_{\mathrm{C} 7 \mathrm{H} 25}(12), \rho_{\mathrm{C} 6 \mathrm{H} 24}(8), v_{\mathrm{C} 8 \mathrm{C} 19}(7)$ \\
\hline 58 & 1222.5 & 7.7 & 1.2 & $\tau_{\mathrm{C} 20 \mathrm{H} 31 \mathrm{H} 32}(23), \tau_{\mathrm{C} 2 \mathrm{H} 22 \mathrm{H} 21}(14), v_{\mathrm{C}_{2} \mathrm{~N} 3}(11), \rho_{\mathrm{C} 19 \mathrm{H} 30}(9)$ \\
\hline 59 & 1255.6 & 73.3 & 3.6 & $\begin{array}{l}\tau_{\mathrm{C} 2 \mathrm{H} 22 \mathrm{H} 21}(14), \rho_{\mathrm{C} 12 \mathrm{H} 26}(11), v_{\mathrm{C} 7 \mathrm{C} 18}(9), \omega_{\mathrm{C} 20 \mathrm{H} 31 \mathrm{H} 32}(9), v_{\mathrm{C} 9 \mathrm{C} 10}(6), \\
\rho_{\mathrm{C} 14 \mathrm{H} 28}(5)\end{array}$ \\
\hline 60 & 1277.2 & 27.7 & 0.5 & $\begin{array}{l}\tau_{\mathrm{C} 2 \mathrm{H} 22 \mathrm{H} 21}(19), \omega_{\mathrm{C} 20 \mathrm{H} 31 \mathrm{H} 32}(15), \tau_{\mathrm{C}_{20 \mathrm{H} 31 \mathrm{H} 32}}(9), \rho_{\mathrm{C} 14 \mathrm{H} 28}(8),{ }^{2} \beta_{\text {trig/R6 }}(8), \\
\rho_{\mathrm{C} 12 \mathrm{H} 26}(6)\end{array}$ \\
\hline 61 & 1292.1 & 537.7 & & $v_{\mathrm{C} 9 \mathrm{C} 10}(12), v_{\mathrm{C} 9 \mathrm{C} 16}(8), v_{\mathrm{C} 8 \mathrm{C} 16}(7), v_{\mathrm{C} 7 \mathrm{C} 18}(6), \rho_{\mathrm{C} 14 \mathrm{H} 28}(6), v_{\mathrm{C} 8 \mathrm{C} 17}(5)$ \\
\hline 62 & 1315.6 & 179.7 & 1.6 & $v_{\mathrm{C}_{11} \mathrm{C} 17}(18), v_{\mathrm{C} 8 \mathrm{C} 17}(14), \rho_{\mathrm{C} 12 \mathrm{H} 26}(8), \rho_{\mathrm{C} 17 \mathrm{O} 4}(7), v_{\mathrm{C} 16 \mathrm{C} 18}(6), \rho_{\mathrm{N} 3 \mathrm{H} 23}(5)$ \\
\hline 63 & 1324.9 & 63.9 & 3.0 & $\begin{array}{l}\omega_{\mathrm{C}_{20} \mathrm{H} 31 \mathrm{H} 32}(23), v_{\mathrm{N} 3 \mathrm{C} 18}(11), \tau_{\mathrm{C}_{2} \mathrm{H} 22 \mathrm{H} 21}(9), \omega_{\mathrm{C}_{2} \mathrm{H} 22 \mathrm{H} 21}(8), \rho_{\mathrm{N}_{3} \mathrm{H} 23}(7), \\
\rho_{\mathrm{C} 6 \mathrm{H} 24}(6)\end{array}$ \\
\hline 64 & 1337.0 & 73.1 & 2.0 & 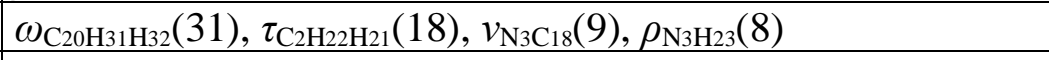 \\
\hline 65 & 1358.5 & 15.3 & 0.8 & \begin{tabular}{|l}
$v_{\mathrm{C} 10 \mathrm{C} 14}(15), v_{\mathrm{C} 10 \mathrm{C} 11}(15), v_{\mathrm{C} 11 \mathrm{C} 12}(14), v_{\mathrm{C} 13 \mathrm{C} 15}(14), v_{\mathrm{C} 12 \mathrm{C} 13}(13)$, \\
$v_{\mathrm{C} 14 \mathrm{C} 15}(13)$
\end{tabular} \\
\hline 66 & 1375.5 & 11.8 & 3.2 & $\begin{array}{l}v_{\mathrm{C} 16 \mathrm{C} 18}(13), v_{\mathrm{C} 8 \mathrm{C} 16}(12), v_{\mathrm{C} 8 \mathrm{C} 19}(10), v_{\mathrm{C} 9 \mathrm{C} 16}(9), v_{\mathrm{C} 6 \mathrm{C} 19}(7), v_{\mathrm{C} 9 \mathrm{C} 10}(6), \\
\rho_{\mathrm{C} 9 \mathrm{O} 5}(6), v_{\mathrm{C} 7 \mathrm{C} 18}(6),{ }^{2} \beta_{2 / R 6}(5)\end{array}$ \\
\hline 67 & 1409.3 & 6.0 & 1.3 & $\omega_{\mathrm{C} 2 \mathrm{H} 22 \mathrm{H} 21}(57), \rho_{\mathrm{C} 7 \mathrm{H} 25}(6), v_{\mathrm{C}_{2} \mathrm{C} 20}(5)$ \\
\hline 68 & 1434.3 & 64.2 & 0.2 & $\begin{array}{l}\rho_{\mathrm{C} 7 \mathrm{H} 25}(21), v_{\mathrm{C} 6 \mathrm{C} 7}(10), v_{\mathrm{C} 8 \mathrm{C} 19}(9), v_{\mathrm{C} 8 \mathrm{C} 16}(7), v_{\mathrm{C} 9 \mathrm{C} 16}(7), \omega_{\mathrm{C} 2 \mathrm{H} 22 \mathrm{H} 21}(7), \\
\rho_{\mathrm{N} 3 \mathrm{H} 23}(6)\end{array}$ \\
\hline
\end{tabular}




\begin{tabular}{|c|c|c|c|c|}
\hline 69 & 1479.4 & 1.0 & 3.4 & $\begin{array}{l}\rho_{\mathrm{C} 15 \mathrm{H} 29}(16), \rho_{\mathrm{C} 13 \mathrm{H} 27}(14), v_{\mathrm{C} 10 \mathrm{C} 14}(7), v_{\mathrm{C} 11 \mathrm{C} 12}(7), \rho_{\mathrm{C} 19 \mathrm{H} 30}(6), \\
v_{\mathrm{C} 8 \mathrm{C} 16}(6)\end{array}$ \\
\hline 70 & 1484.6 & 15.5 & 0.8 & $\delta_{1 / \mathrm{C} 20 \mathrm{H} 31 \mathrm{H} 32}(84)$ \\
\hline 71 & 1489.4 & 22.1 & 0.9 & $\begin{array}{l}\rho_{\mathrm{C} 19 \mathrm{H} 30}(12), v_{\mathrm{C} 8 \mathrm{C} 16}(10), \rho_{\mathrm{C} 6 \mathrm{H} 24}(8), v_{\mathrm{C} 7 \mathrm{C} 18}(6), \delta_{1 / \mathrm{C} 20 \mathrm{H} 31 \mathrm{H} 32}(6), \\
\rho_{\mathrm{C} 13 \mathrm{H} 27}(5), v_{\mathrm{N} 3 \mathrm{C} 18}(5)\end{array}$ \\
\hline 72 & 1507.6 & 9.8 & 2.0 & $\rho_{\mathrm{C} 14 \mathrm{H} 28}(20), \rho_{\mathrm{C} 12 \mathrm{H} 26}(19), v_{\mathrm{C} 10 \mathrm{C} 11}(16), v_{\mathrm{C} 13 \mathrm{C} 15}(8), \rho_{\mathrm{C} 15 \mathrm{H} 29}(5)$ \\
\hline 73 & 1513.9 & 59.2 & 1.0 & $\delta_{1 / \mathrm{C}_{2} \mathrm{H}_{22} \mathrm{H} 21}(88)$ \\
\hline 74 & 1548.4 & 239.2 & 0.8 & $\rho_{\mathrm{N} 3 \mathrm{H} 23}(21), v_{\mathrm{N} 3 \mathrm{C} 18}(17), v_{\mathrm{C} 6 \mathrm{C} 19}(17), \rho_{\mathrm{C} 6 \mathrm{H} 24}(11), v_{\mathrm{C} 8 \mathrm{C} 16}(6), v_{\mathrm{C} 6 \mathrm{C} 7}(6)$ \\
\hline 75 & 1608.0 & 125.6 & 8.7 & $\begin{array}{l}\rho_{\mathrm{N} 3 \mathrm{H} 23}(20), v_{\mathrm{C} 6 \mathrm{C} 19}(14), v_{\mathrm{C} 16 \mathrm{C} 18}(12), v_{\mathrm{C} 8 \mathrm{C} 19}(10),{ }^{1} \beta_{2 / R 6}(9), \\
v_{\mathrm{C} 7 \mathrm{C} 18}(7), \rho_{\mathrm{C} 18 \mathrm{~N} 3}(6)\end{array}$ \\
\hline 76 & 1612.6 & 21.7 & 2.1 & $\begin{array}{l}v_{\mathrm{C} 13 \mathrm{C} 15}(21), v_{\mathrm{C} 10 \mathrm{C} 11}(20), v_{\mathrm{O} 5 \mathrm{C} 9}(11), v_{\mathrm{C} 12 \mathrm{C} 13}(10),{ }^{3} \beta_{3 / R 6}(9), \\
v_{\mathrm{C} 10 \mathrm{C} 14}(8), \rho_{\mathrm{C} 13 \mathrm{H} 27}(7)\end{array}$ \\
\hline 77 & 1626.3 & 42.9 & 17.6 & $v_{\mathrm{C} 6 \mathrm{C} 7}(11), v_{\mathrm{O} 5 \mathrm{C} 9}(10), v_{\mathrm{C}_{14} \mathrm{C}_{15}}(9), v_{\mathrm{C} 8 \mathrm{C} 19}(9), v_{\mathrm{C}_{11} \mathrm{C}_{12}}(7)$ \\
\hline 78 & 1630.5 & 245.4 & 6.2 & $\begin{array}{l}v_{\mathrm{C} 14 \mathrm{C} 15}(11), v_{\mathrm{C} 6 \mathrm{C} 7}(10), v_{\mathrm{C} 12 \mathrm{C} 13}(9), v_{\mathrm{C} 11 \mathrm{C} 12}(8), v_{\mathrm{C} 10 \mathrm{C} 14}(6), v_{\mathrm{O} 4 \mathrm{C} 17}(6), \\
3^{3} \beta_{2 / R 6}(5)\end{array}$ \\
\hline 79 & 1672.5 & 215.3 & 27.6 & $v_{\mathrm{O} 5 \mathrm{C} 9}(45),{ }^{2} \beta_{\text {trig/R6}}(6),{ }^{2} \beta_{3 / R 6}(6)$ \\
\hline 80 & 1720.4 & 183.5 & 26.3 & $v_{\mathrm{O} 4 \mathrm{C} 17}(71),{ }^{2} \beta_{\text {trig/R6}}(9)$ \\
\hline 81 & 2672.3 & 5.9 & 15.5 & $v_{\mathrm{S} 1 \mathrm{H} 33}(100)$ \\
\hline 82 & 2983.0 & 63.7 & 11.2 & $v_{\mathrm{C} 2 \mathrm{H} 22}(60), v_{\mathrm{C}_{2} \mathrm{H} 21}(39)$ \\
\hline 83 & 3006.3 & 17.7 & 5.5 & $v_{\mathrm{C}_{2} \mathrm{H} 21}(59), v_{\mathrm{C}_{2} \mathrm{H}_{22}}(39)$ \\
\hline 84 & 3061.9 & 25.3 & 7.6 & $v_{\mathrm{C} 20 \mathrm{H} 32}(62), v_{\mathrm{C} 20 \mathrm{H} 31}(36)$ \\
\hline 85 & 3113.7 & 9.5 & 5.1 & $v_{\mathrm{C}_{20 \mathrm{H} 31}}(63), v_{\mathrm{C}_{20 \mathrm{H}} 32}(36)$ \\
\hline 86 & 3169.6 & 3.5 & 4.5 & $v_{\mathrm{C} 15 \mathrm{H} 29}(48), v_{\mathrm{C} 13 \mathrm{H} 27}(46)$ \\
\hline 87 & 3172.0 & 12.5 & 8.1 & $v_{\mathrm{C} 6 \mathrm{H} 24}(90), v_{\mathrm{C} 7 \mathrm{H} 25}(6)$ \\
\hline 88 & 3184.5 & 13.8 & 14.5 & $v_{\mathrm{C} 15 \mathrm{H} 29}(43), v_{\mathrm{C} 13 \mathrm{H} 27}(42), v_{\mathrm{C} 12 \mathrm{H} 26}(8), v_{\mathrm{C} 14 \mathrm{H} 28}(5)$ \\
\hline 89 & 3201.5 & 3.8 & 6.8 & $v_{\mathrm{C}_{12} \mathrm{H} 26}(82), v_{\mathrm{C} 14 \mathrm{H} 28}(9), v_{\mathrm{C} 13 \mathrm{H} 27}(9)$ \\
\hline 90 & 3205.3 & 10.3 & 7.0 & $v_{\mathrm{C} 7 \mathrm{H} 25}(89), v_{\mathrm{C} 19 \mathrm{H} 30}(5)$ \\
\hline 91 & 3205.7 & 7.8 & 13.5 & $v_{\mathrm{C} 14 \mathrm{H} 28}(82), v_{\mathrm{C} 15 \mathrm{H} 29}(8), v_{\mathrm{C} 12 \mathrm{H} 26}(6)$ \\
\hline 92 & 3212.7 & 4.2 & 13.1 & $v_{\mathrm{C} 19 \mathrm{H} 30}(91)$ \\
\hline 93 & 3452.7 & 79.1 & 31.4 & $v_{\mathrm{N} 3 \mathrm{H} 23}(99)$ \\
\hline
\end{tabular}


Table S.16. Frequencies of normal vibrations, IR and Raman intensities and PEDs for $T-G G$ conformer calculated at the B3LYP/6-311++G(d,p) level of theory.

\begin{tabular}{|c|c|c|c|c|}
\hline $\mathbf{N r}$ & $\begin{array}{l}\text { Freq. } \\
{\left[\mathrm{cm}^{-1}\right]}\end{array}$ & IR Int. & $\begin{array}{c}\text { Raman } \\
\text { Int. }\end{array}$ & PED $($ contributions $>5 \%)$ \\
\hline 1 & 20.8 & 0.7 & 14.3 & $\tau_{\mathrm{C} 2 \mathrm{~N} 3}(56),{ }^{1} \tau_{3 / R 6}(9), \omega_{\mathrm{N} 3 \mathrm{H} 23}(8)$ \\
\hline 2 & 30.5 & 1.3 & 8.0 & $\tau_{\mathrm{N} 3 \mathrm{C} 18}(36), \omega_{\mathrm{N} 3 \mathrm{H} 23}(29),{ }^{2} \tau_{3 / R 6}(9),{ }^{2} \tau_{2 / R 6}(6)$ \\
\hline 3 & 45.4 & 3.9 & 0.8 & ${ }^{2} \tau_{3 / R \sigma}(43),{ }^{2} \tau_{2 / R 6}(17), \omega_{\mathrm{N}_{3} \mathrm{H} 23}(13),{ }^{1} \tau_{3 / R 6}(5)$ \\
\hline 4 & 70.2 & 0.2 & 0.2 & ${ }^{2} \tau_{2 / R \sigma}(23),{ }^{1} \tau_{3 / R \sigma}(16),{ }^{1} \tau_{2 / R \sigma}(6),{ }^{2} \tau_{3 / R 6}(6), \tau_{\mathrm{N} 3 \mathrm{C} 18}(6)$ \\
\hline 5 & 108.3 & 0.9 & 0.4 & \begin{tabular}{|l}
$\beta_{\mathrm{C} 18 \mathrm{~N} 3 \mathrm{C} 2_{2}}(18), \omega_{\mathrm{N} 3 \mathrm{H} 23}(16),{ }^{2} \tau_{2 / R 6}(14), \rho_{\mathrm{C} 18 \mathrm{~N} 3_{3}}(13), \delta_{2 / \mathrm{C} 2 \mathrm{H} 22 \mathrm{H} 21}(10)$, \\
$\tau_{\mathrm{N}_{3} \mathrm{C} 18}(6)$
\end{tabular} \\
\hline 6 & 119.9 & 0.4 & 1.0 & ${ }^{2} \tau_{1 / R \sigma}(38),{ }^{3} \tau_{2 / R \sigma}(15),{ }^{2} \tau_{2 / R 6}(10),{ }^{3} \tau_{3 / R \sigma}(7)$ \\
\hline 7 & 149.0 & 2.4 & 0.7 & $\omega_{\mathrm{N} 3 \mathrm{H} 23}(27), \tau_{\mathrm{C}_{2} \mathrm{C} 20}(25),{ }^{2} \tau_{1 / R 6}(11), \delta_{2 / \mathrm{C} 20 \mathrm{H} 31 \mathrm{H} 32}(9),{ }^{1} \tau_{2 / R 6}(7)$ \\
\hline 8 & 166.0 & 0.4 & 0.0 & $\begin{array}{l}{ }^{4} b f l_{R R}(24),{ }^{2} b f l_{R R}(22), \tau_{N 3 C l 8}(10),{ }^{2} \tau_{3 / R 6}(8), \omega_{C 17 O 4}(6),{ }^{I} \tau_{3 / R 6}(5), \\
\omega_{C 9 O 5}(5)\end{array}$ \\
\hline 9 & 202.2 & 0.5 & 1.3 & ${ }^{1}{ }_{\tau_{3 / R 6}}(24),{ }^{1} \tau_{2 / R 6}(20),{ }^{4} b f l_{R R}(17), \omega_{C 19 H 30}(6)$ \\
\hline 10 & 209.6 & 16.5 & 0.2 & $\tau_{\mathrm{S} 1 \mathrm{C} 20_{2}}(60), \delta_{2 / \mathrm{C} 2 \mathrm{H} 22 \mathrm{H} 21}(9)$ \\
\hline 11 & 241.2 & 1.4 & 0.2 & $\tau_{\mathrm{S} 1 \mathrm{C} 20}(21), \beta_{\mathrm{C} 18 \mathrm{~N} 3 \mathrm{C} 2}(12),{ }^{3} \beta_{2 / R 6}(10), \delta_{2 / \mathrm{C}_{2} \mathrm{H} 22 \mathrm{H} 21}(6)$ \\
\hline 12 & 260.6 & 2.8 & 1.4 & $\begin{array}{l}\delta_{2 / \mathrm{C} 20 \mathrm{H} 31 \mathrm{H} 32}(15), \tau_{\mathrm{C} 2 \mathrm{C} 20}(14), \tau_{\mathrm{S} 1 \mathrm{C} 20}(11), \beta_{\mathrm{C} 18 \mathrm{~N} 3 \mathrm{C} 2}(9), \delta_{2 / \mathrm{C} 2 \mathrm{H} 22 \mathrm{H} 21}(8), \\
{ }^{2} \beta_{2 / R 6}(6)\end{array}$ \\
\hline 13 & 270.9 & 1.2 & 0.5 & $\begin{array}{l}{ }^{2} b f l_{R R}(26), \omega_{N_{3} H_{23}}(12), \omega_{C 18 N_{3}}(8),{ }^{l} \tau_{2 / R 6}(7),{ }^{l} \tau_{3 / R 6}(5), \omega_{C 7 H_{25}}(5), \\
\tau_{C 2 C 20}(5),{ }^{l} \tau_{1 / R 6}(5)\end{array}$ \\
\hline 14 & 318.2 & 5.2 & 0.0 & ${ }^{2} \beta_{2 / R 6}(37), \delta_{2 / \mathrm{C}_{2} \mathrm{H} 22 \mathrm{H} 21}(8), \delta_{2 / \mathrm{C}_{20} \mathrm{H} 31 \mathrm{H} 32}(7), \beta_{\mathrm{C} 18 \mathrm{~N} 3 \mathrm{C} 2_{2}}(6)$ \\
\hline 15 & 343.6 & 1.5 & 0.2 & ${ }^{2} \beta_{3 / R 6}(27), \rho_{\mathrm{C} 17 \mathrm{O} 4}(19), v_{\mathrm{C} 8 \mathrm{C} 17}(9), \rho_{\mathrm{C} 18 \mathrm{~N} 3}(8),{ }^{1} \beta_{2 / R 6}(5)$ \\
\hline 16 & 372.3 & 7.5 & 1.3 & $\begin{array}{l}{ }^{2} \beta_{3 / R 6}(20), \delta_{2 / \mathrm{C}_{20 \mathrm{H} 31 \mathrm{H} 32}}(14),{ }^{1} \beta_{3 / R 6}(13), v_{\mathrm{C} 11 \mathrm{C} 17}(10), v_{\mathrm{C} 9 \mathrm{C} 16}(7), \\
\rho_{\mathrm{C} 17 \mathrm{O} 4}(5)\end{array}$ \\
\hline 17 & 426.4 & 23.5 & 0.3 & $\rho_{\mathrm{C} 9 \mathrm{O} 5}(30), \rho_{\mathrm{C} 17 \mathrm{O} 4}(18),{ }^{1} \beta_{3 / R 6}(10), \delta_{2 / \mathrm{C}_{20} \mathrm{H} 31 \mathrm{H} 32}(5), v_{\mathrm{C} 9 \mathrm{C} 16}(5)$ \\
\hline 18 & 426.5 & 0.4 & 0.1 & ${ }^{3} \tau_{3 / R \sigma}(60),{ }^{4} b f l_{R R}(14)$ \\
\hline 19 & 431.4 & 0.5 & 0.5 & $\begin{array}{l}{ }^{3} \tau_{2 / R \sigma}(13), \delta_{2 / \mathrm{C}_{2} \mathrm{H}_{22} \mathrm{H}_{21}}(11), \delta_{2 / \mathrm{C}_{20 \mathrm{H} 31 \mathrm{H} 32}}(10), \rho_{\mathrm{C} 18 \mathrm{~N} 3}(9), \rho_{\mathrm{C} 9 \mathrm{O} 5}(6), \\
{ }^{1} \tau_{3 / R \sigma}(6),{ }_{2} \tau_{2 / R \sigma}(6),{ }^{2} \beta_{3 / R 6}(6)\end{array}$ \\
\hline 20 & 434.8 & 0.1 & 0.4 & ${ }^{3} \tau_{2 / R 6}(25),{ }^{1} \tau_{2 / R 6}(16),{ }^{1} \tau_{3 / R 6}(12),{ }^{2} \tau_{1 / R 6}(5)$ \\
\hline 21 & 472.6 & 2.6 & 1.3 & ${ }^{2} \beta_{2 / R 6}(34),{ }^{1} \beta_{2 / R 6}(12), \rho_{\mathrm{C} 18 \mathrm{~N} 3}(11),{ }^{3} \beta_{2 / R 6}(7),{ }^{3} \beta_{3 / R 6}(6)$ \\
\hline 22 & 486.3 & 0.8 & 9.4 & $\begin{array}{l}{ }^{2} \beta_{3 / R 6}(27),{ }^{1} \beta_{3 / R \sigma}(8),{ }^{1} \beta_{2 / R 6}(7),{ }^{3} \beta_{2 / R 6}(6), \rho_{\mathrm{C} 18 \mathrm{~N} 3}(6), \delta_{2 / \mathrm{C} 2 \mathrm{H} 22 \mathrm{H} 21}(5), \\
{ }^{2} \beta_{2 / R 6}(5)\end{array}$ \\
\hline 23 & 491.0 & 2.3 & 0.3 & ${ }^{3} \tau_{2 / R \sigma}(23),{ }^{1} \tau_{3 / R \sigma}(23),{ }^{1} \tau_{2 / R \sigma}(11),{ }^{2} \tau_{2 / R 6}(9),{ }^{1} \tau_{1 / R 6}(5),{ }^{3} \tau_{3 / R \sigma}(5)$ \\
\hline 24 & 556.9 & 7.9 & 0.1 & $\omega_{\mathrm{C} 18 \mathrm{~N} 3}(29),{ }^{1} \tau_{3 / R \sigma}(14),{ }^{1} \tau_{2 / R \sigma}(13),{ }^{1}{ }_{1 / R \sigma}(10),{ }^{2} b f l_{R R}(6)$ \\
\hline 25 & 617.6 & 6.4 & 0.6 & ${ }^{2} \beta_{\text {trig/R6}}(25),{ }_{2 / R 6}^{1}(24),{ }^{3} \beta_{2 / R 6}(15),{ }^{1} \beta_{3 / R 6}(7)$ \\
\hline 26 & 639.8 & 3.1 & 1.2 & ${ }^{2} \beta_{\text {trig/R } 6}(24),{ }^{3} \beta_{3 / R 6}(13),{ }^{1} \beta_{3 / R 6}(13), v_{\mathrm{S} 1 \mathrm{C} 20}(7)$ \\
\hline 27 & 649.6 & 55.1 & 0.8 & $\tau_{\mathrm{N} 3 \mathrm{C} 18}(29), \omega_{\mathrm{N} 3 \mathrm{H} 23}(27), v_{\mathrm{S}_{1} \mathrm{C} 20}(8)$ \\
\hline 28 & 655.1 & 1.7 & 1.1 & $v_{\mathrm{S}_{1} \mathrm{C}_{20}}(62), \delta_{2 / \mathrm{C}_{20} \mathrm{H} 31 \mathrm{H} 32}(5),{ }^{3} \beta_{3 / R 6}(5)$ \\
\hline 29 & 673.7 & 9.2 & 0.1 & ${ }^{3} \tau_{1 / R 6}(38),{ }^{1} \tau_{1 / R 6}(18), \omega_{\mathrm{C} 9 \mathrm{O} 5}(12), \omega_{\mathrm{C} 18 \mathrm{~N} 3}(11)$ \\
\hline 30 & 687.2 & 6.0 & 1.8 & ${ }^{3} \beta_{3 / R 6}(31),{ }^{1} \beta_{3 / R 6}(14), \beta_{\mathrm{C} 18 \mathrm{~N} 3 \mathrm{C} 2}(6), v_{\mathrm{C} 10 \mathrm{C} 11}(5)$ \\
\hline 31 & 710.7 & 3.1 & 0.4 & ${ }^{3} \beta_{2 / R 6}(24), \rho_{\mathrm{C} 17 \mathrm{O} 4}(16), \rho_{\mathrm{C} 9 \mathrm{O} 5}(15),{ }^{1} \beta_{2 / R 6}(12), \rho_{\mathrm{C} 18 \mathrm{~N} 3}(6)$ \\
\hline 32 & 723.7 & 36.3 & 0.0 & ${ }^{3} \tau_{1 / R 6}(28),{ }^{1} \tau_{1 / R 6}(27), \omega_{\mathrm{C} 6 \mathrm{H} 24}(9), \omega_{\mathrm{C} 15 \mathrm{H} 29}(7), \omega_{\mathrm{C} 17 \mathrm{O} 4}(6)$ \\
\hline 33 & 746.6 & 55.4 & 0.0 & $\begin{array}{l}{ }^{\mathrm{l}} \tau_{1 / R 6}(14), \omega_{\mathrm{C} 13 \mathrm{H} 27}(14), \omega_{\mathrm{C} 18 \mathrm{~N} 3}(13), \omega_{\mathrm{C} 9 \mathrm{O} 5}(9), \omega_{\mathrm{C} 17 \mathrm{O} 4}(8), \\
\omega_{\mathrm{C} 14 \mathrm{H} 28}(7), \omega_{\mathrm{C} 15 \mathrm{H} 29}(7),{ }^{3} \tau_{1 / R 6}(7)\end{array}$ \\
\hline 34 & 759.5 & 0.1 & 0.7 & $\rho_{\mathrm{C} 20 \mathrm{H} 31 \mathrm{H} 32}(47), \beta_{\mathrm{C} 20 \mathrm{~S} 1 \mathrm{H} 33}(29)$ \\
\hline 35 & 785.0 & 8.0 & 0.0 & $\begin{array}{l}{ }^{3} \tau_{1 / R 6}(19),{ }^{2} \tau_{1 / R 6}(14), \omega_{\mathrm{C} 7 \mathrm{H} 25}(13), \omega_{\mathrm{C} 19 \mathrm{H} 30}(11), \omega_{\mathrm{C} 6 \mathrm{H} 24}(10), \\
\omega_{\mathrm{C} 9 \mathrm{O} 5}(10), \omega_{\mathrm{C} 17 \mathrm{O} 4}(10)\end{array}$ \\
\hline 36 & 799.9 & 1.1 & 0.5 & ${ }^{1} \beta_{\text {trig/R6 }}(19),{ }^{3} \beta_{2 / R 6}(11),{ }^{1} \beta_{3 / R \sigma}(11),{ }^{3} \beta_{\text {trig/R6 }}(9),{ }^{2} \beta_{\text {trig/R6 }}(8)$, \\
\hline
\end{tabular}




\begin{tabular}{|c|c|c|c|c|}
\hline & & & & ${ }^{1} \beta_{2 / R 6}(6), v_{\mathrm{C} 9 \mathrm{C} 10}(5)$ \\
\hline 37 & 810.3 & 1.0 & 0.1 & $\begin{array}{l}\omega_{\mathrm{C} 9 \mathrm{O} 5}(26), \omega_{\mathrm{C} 13 \mathrm{H} 27}(14),{ }^{1} \tau_{1 / R 6}(11), \omega_{\mathrm{C} 18 \mathrm{~N} 3}(9), \omega_{\mathrm{C} 15 \mathrm{H} 29}(8), \\
{ }^{2} \tau_{1 / R 6}(7)\end{array}$ \\
\hline 38 & 827.2 & 28.2 & 0.0 & $\begin{array}{l}\omega_{\mathrm{C} 7 \mathrm{H} 25}(22),{ }^{1} \tau_{1 / R 6}(18), \omega_{\mathrm{C} 17 \mathrm{O} 4}(17), \omega_{\mathrm{C} 18 \mathrm{~N} 3}(10),{ }^{2} \tau_{1 / R 6}(8), \\
\omega_{\mathrm{C} 15 \mathrm{H} 29}(6), \omega_{\mathrm{C} 6 \mathrm{H} 24}(5)\end{array}$ \\
\hline 39 & 883.7 & 26.1 & 2.2 & $v_{\mathrm{C} 7 \mathrm{C} 18}(10), \beta_{\mathrm{C} 20 \mathrm{~S} 1 \mathrm{H} 33}(10), \rho_{\mathrm{C} 17 \mathrm{O} 4}(9), v_{\mathrm{C} 16 \mathrm{C} 18}(7), v_{\mathrm{N}_{3} \mathrm{C} 18}(6),{ }^{1} \beta_{2 / R 6}(5)$ \\
\hline 40 & 909.0 & 0.4 & 0.0 & $\omega_{\mathrm{C} 19 \mathrm{H} 30}(33), \omega_{\mathrm{C} 7 \mathrm{H} 25}(23), \omega_{\mathrm{C} 12 \mathrm{H} 26}(11), \omega_{\mathrm{C} 14 \mathrm{H} 28}(11), \omega_{\mathrm{C} 15 \mathrm{H} 29}(6)$ \\
\hline 41 & 915.9 & 0.5 & 0.0 & $\begin{array}{l}\omega_{\mathrm{C} 14 \mathrm{H} 28}(20), \omega_{\mathrm{C} 12 \mathrm{H} 26}(18), \omega_{\mathrm{C} 19 \mathrm{H} 30}(17), \omega_{\mathrm{C} 7 \mathrm{H} 25}(11), \omega_{\mathrm{C} 13 \mathrm{H} 27}(9), \\
\omega_{\mathrm{C} 15 \mathrm{H} 29}(7)\end{array}$ \\
\hline 42 & 929.3 & 3.8 & 1.4 & ${ }^{1} \beta_{\text {trig/R6 }}(24),{ }^{3} \beta_{\text {trig/R6 }}(23), \rho_{\mathrm{C} 955}(15), v_{\mathrm{C} 16 \mathrm{C} 18}(8)$ \\
\hline 43 & 950.2 & 15.9 & 0.1 & $v_{\mathrm{C}_{2} \mathrm{C} 20}(21), \beta_{\mathrm{C}_{20} \mathrm{~S} 1 \mathrm{H} 33}(17), v_{\mathrm{C} 2 \mathrm{~N} 3}(10), \rho_{\mathrm{C} 17 \mathrm{O} 4}(6)$ \\
\hline 44 & 983.9 & 1.3 & 0.4 & $\rho_{\mathrm{C}_{2} \mathrm{H} 22 \mathrm{H} 21}(45), \beta_{\mathrm{C}_{20} \mathrm{~S}_{1 \mathrm{H} 33}}(10), v_{\mathrm{C}_{2} \mathrm{C} 20}(10), \tau_{\mathrm{C} 20 \mathrm{H} 31 \mathrm{H} 32}(5)$ \\
\hline 45 & 999.5 & 0.2 & 0.0 & $\begin{array}{l}\omega_{\mathrm{C} 6 \mathrm{H} 24}(44), \omega_{\mathrm{C} 19 \mathrm{H} 30}(18), \omega_{\mathrm{C} 7 \mathrm{H} 25}(9), \omega_{\mathrm{C} 12 \mathrm{H} 26}(7),{ }^{1} \tau_{1 / R 6}(6), \\
\omega_{\mathrm{C} 14 \mathrm{H} 28}(6)\end{array}$ \\
\hline 46 & 1001.7 & 1.8 & 0.0 & $\begin{array}{l}\omega_{\mathrm{C} 12 \mathrm{H} 26}(27), \omega_{\mathrm{C} 14 \mathrm{H} 28}(24), \omega_{\mathrm{C} 6 \mathrm{H} 24}(12), \omega_{\mathrm{C} 13 \mathrm{H} 27}(9), \omega_{\mathrm{C} 15 \mathrm{H} 29}(9), \\
\omega_{\mathrm{C} 19 \mathrm{H} 30}(6)\end{array}$ \\
\hline 47 & 1016.1 & 0.0 & 0.0 & $\omega_{\mathrm{C} 15 \mathrm{H} 29}(31), \omega_{\mathrm{C} 13 \mathrm{H} 27}(28), \omega_{\mathrm{C} 14 \mathrm{H} 28}(15), \omega_{\mathrm{C} 12 \mathrm{H} 26}(13),{ }^{3} \tau_{1 / R 6}(12)$ \\
\hline 48 & 1029.0 & 62.6 & .4 & $v_{\mathrm{C} 8 \mathrm{C} 17}(14), v_{\mathrm{C} 13 \mathrm{C} 15}(9), v_{\mathrm{C} 2 \mathrm{C} 20}(6), \beta_{\mathrm{C}_{20} \mathrm{~S} 1 \mathrm{H} 33}(6), v_{\mathrm{C} 12 \mathrm{C} 13}(6), v_{\mathrm{C} 11 \mathrm{C} 12}(5)$ \\
\hline 49 & 1059.2 & 0.8 & 4.3 & $v_{\mathrm{C} 13 \mathrm{C} 15}(28), \rho_{\mathrm{C} 12 \mathrm{H} 26}(11), v_{\mathrm{C} 14 \mathrm{C} 15}(11), \rho_{\mathrm{C} 14 \mathrm{H} 28}(11), v_{\mathrm{C} 12 \mathrm{C} 13}(8)$ \\
\hline 50 & 1067.8 & 20.0 & 0.3 & $\begin{array}{l}v_{\mathrm{C}_{2} 20}(29), \rho_{\mathrm{C}_{2} \mathrm{H} 22 \mathrm{H} 21}(19), \rho_{\mathrm{C}_{20} \mathrm{H} 31 \mathrm{H} 32}(16), \beta_{\mathrm{C}_{20 \mathrm{~S} 1 \mathrm{H} 33}}(9) \\
\delta_{2 / \mathrm{C} 2 \mathrm{H} 22 \mathrm{H} 21}(6)\end{array}$ \\
\hline 51 & 1096.5 & 16.6 & 1.4 & $v_{\mathrm{C} 6 \mathrm{C} 19}(31), \rho_{\mathrm{C} 19 \mathrm{H} 30}(16),{ }^{1} \beta_{\text {trig/R6 }}(13), v_{\mathrm{C} 6 \mathrm{C} 7}(9), \rho_{\mathrm{C} 7 \mathrm{H} 25}(6)$ \\
\hline 52 & 1113.2 & 1.1 & 0.3 & $\begin{array}{l}{ }^{3} \beta_{\text {trig/R6 }}(37), v_{\mathrm{C} 12 \mathrm{C} 13}(8), v_{\mathrm{C} 14 \mathrm{C} 15}(8), \rho_{\mathrm{C} 13 \mathrm{H} 27}(8), \rho_{\mathrm{C} 15 \mathrm{H} 29}(7), \\
v_{\mathrm{C} 9 \mathrm{C} 10}(6), \rho_{\mathrm{C} 14 \mathrm{H} 28}(5), v_{\mathrm{C} 11 \mathrm{C} 17}(5), \rho_{\mathrm{C} 12 \mathrm{H} 26}(5)\end{array}$ \\
\hline 53 & 1140.8 & 5.5 & 13 & $v_{\mathrm{C}_{2} \mathrm{~N} 3}(43), \tau_{\mathrm{C} 20 \mathrm{H} 31 \mathrm{H} 32}(8), \rho_{\mathrm{C} 18 \mathrm{~N} 3}(6), v_{\mathrm{C}_{2} \mathrm{C} 20}(6)$ \\
\hline 54 & 1176.5 & 3.3 & 4.4 & $\begin{array}{l}\rho_{\mathrm{C} 13 \mathrm{H} 27}(19), \rho_{\mathrm{C} 12 \mathrm{H} 26}(13), v_{\mathrm{C} 9 \mathrm{C} 16}(8), v_{\mathrm{C} 9 \mathrm{C} 10}(7),{ }^{1} \beta_{\text {trig/R6 }}(6), \\
{ }^{3} \beta_{\text {trig/R6 }}(5)\end{array}$ \\
\hline 55 & 1184.1 & 17.1 & 0.4 & $\begin{array}{l}\rho_{\mathrm{C} 15 \mathrm{H} 29}(29), v_{\mathrm{C} 14 \mathrm{C} 15}(9), \rho_{\mathrm{C} 14 \mathrm{H} 28}(9),{ }^{1} \beta_{\text {trig/R6 }}(7),{ }^{3} \beta_{\text {trig/R6 }}(7), \\
v_{\mathrm{C} 9 \mathrm{C} 16}(6), \rho_{\mathrm{C} 13 \mathrm{H} 27}(6)\end{array}$ \\
\hline 56 & 1197.1 & 30.1 & 4 & $\rho_{\mathrm{C} 6 \mathrm{H} 24}(29), \rho_{\mathrm{C} 7 \mathrm{H} 25}(14), v_{\mathrm{C} 6 \mathrm{C} 7}(7), \rho_{\mathrm{C} 13 \mathrm{H} 27}(7)$ \\
\hline 57 & 1213.9 & 4.5 & 7 & $\rho_{\mathrm{C} 19 \mathrm{H} 30}(24), \rho_{\mathrm{C} 7 \mathrm{H} 25}(11), v_{\mathrm{C} 8 \mathrm{C} 19}(9), v_{\mathrm{C} 11 \mathrm{C} 17}(7), \rho_{\mathrm{C} 6 \mathrm{H} 24}(6), \rho_{\mathrm{C} 14 \mathrm{H} 28}(5)$ \\
\hline 58 & 1228.4 & 31.3 & 1.0 & $\tau_{\mathrm{C} 20 \mathrm{H} 31 \mathrm{H} 32}(27), \tau_{\mathrm{C}_{2} \mathrm{H} 22 \mathrm{H} 21}(27), \omega_{\mathrm{C} 20 \mathrm{H} 31 \mathrm{H} 32}(8), v_{\mathrm{C} 2 \mathrm{~N} 3}(7), \rho_{\mathrm{C} 2 \mathrm{H} 22 \mathrm{H} 21}(6)$ \\
\hline 59 & 1259.2 & 33.8 & 3.3 & $\begin{array}{l}\rho_{\mathrm{C} 12 \mathrm{H} 26}(13), \tau_{\mathrm{C} 2 \mathrm{H} 22 \mathrm{H} 21}(12), v_{\mathrm{C} 7 \mathrm{C} 18}(7), \rho_{\mathrm{C} 14 \mathrm{H} 28}(7), \omega_{\mathrm{C} 20 \mathrm{H} 31 \mathrm{H} 32}(6), \\
v_{\mathrm{C} 11 \mathrm{C} 12}(6),{ }^{2} \beta_{\text {trig/R6}}(6)\end{array}$ \\
\hline 60 & 1281.0 & 17.1 & 0.9 & $\tau_{\mathrm{C} 20 \mathrm{H} 31 \mathrm{H} 32}(18), \tau_{\mathrm{C}_{2} \mathrm{H} 22 \mathrm{H} 21}(16), \omega_{\mathrm{C} 20 \mathrm{H} 31 \mathrm{H} 32}(11),{ }^{2} \beta_{\text {trig/R6}}(7), \rho_{\mathrm{Cl} 14 \mathrm{H} 28}(6)$ \\
\hline 61 & 1291.6 & 596.7 & 9.2 & $\begin{array}{l}v_{\mathrm{C} 9 \mathrm{C} 10}(12), v_{\mathrm{C} 9 \mathrm{C} 16}(8), v_{\mathrm{C} 8 \mathrm{C} 16}(7), v_{\mathrm{C} 7 \mathrm{C} 18}(7), \tau_{\mathrm{C} 20 \mathrm{H} 31 \mathrm{H} 32}(6), v_{\mathrm{C} 8 \mathrm{C} 17}(6), \\
v_{\mathrm{C} 11 \mathrm{C} 17}(5), \rho_{\mathrm{C} 14 \mathrm{H} 28}(5)\end{array}$ \\
\hline 62 & 1316.5 & 151.7 & 1 & $v_{\mathrm{C} 11 \mathrm{C} 17}(18), v_{\mathrm{C} 8 \mathrm{C} 17}(14), \rho_{\mathrm{C} 12 \mathrm{H} 26}(8), \rho_{\mathrm{C} 17 \mathrm{O} 4}(7),{ }^{2} \beta_{2 / R 6}(6)$ \\
\hline 63 & 1327.3 & 75.3 & 3.3 & $\omega_{\mathrm{C} 20 \mathrm{H} 31 \mathrm{H} 32}(33), v_{\mathrm{N} 3 \mathrm{C} 18}(10), \tau_{\mathrm{C}_{2} \mathrm{H} 22 \mathrm{H} 21}(9), \omega_{\mathrm{C} 2 \mathrm{H} 22 \mathrm{H} 21}(8), \rho_{\mathrm{N} 3 \mathrm{H} 23}(6)$ \\
\hline 64 & 1338.0 & 72.5 & 2.1 & $\begin{array}{l}\omega_{\mathrm{C} 20 \mathrm{H} 31 \mathrm{H} 32}(24), \tau_{\mathrm{C} 2 \mathrm{H} 22 \mathrm{H} 21}(15), v_{\mathrm{N} 3 \mathrm{C} 18}(10), \rho_{\mathrm{N}_{3} \mathrm{H} 23}(9), v_{\mathrm{C} 16 \mathrm{C} 18}(5), \\
\rho_{\mathrm{C} 6 \mathrm{H} 24}(5)\end{array}$ \\
\hline 65 & 1358.8 & 16.6 & 0.9 & $\begin{array}{l}v_{\mathrm{C} 10 \mathrm{C} 14}(15), v_{\mathrm{C} 10 \mathrm{C} 11}(15), v_{\mathrm{C} 11 \mathrm{C} 12}(14), v_{\mathrm{C} 13 \mathrm{C} 15}(14), v_{\mathrm{C} 12 \mathrm{C} 13}(13), \\
v_{\mathrm{C} 14 \mathrm{C} 15}(13)\end{array}$ \\
\hline 66 & 1377.3 & 12.5 & 3.3 & $\begin{array}{l}v_{\mathrm{C} 16 \mathrm{C} 18}(13), v_{\mathrm{C} 8 \mathrm{C} 16}(12), v_{\mathrm{C} 8 \mathrm{C} 19}(10), v_{\mathrm{C} 9 \mathrm{C} 16}(8), v_{\mathrm{C} 6 \mathrm{C} 19}(7), v_{\mathrm{C} 9 \mathrm{C} 10}(6), \\
\rho_{\mathrm{C} 9 \mathrm{O} 5}(6), v_{\mathrm{C} 7 \mathrm{C} 18}(5)\end{array}$ \\
\hline 67 & 1405.2 & 9.3 & 1.3 & $\omega_{\mathrm{C} 2 \mathrm{H} 22 \mathrm{H} 21}(58), v_{\mathrm{C} 16 \mathrm{C} 18}(6)$ \\
\hline 68 & 1434.1 & 55.7 & 0.3 & $\rho_{\mathrm{C} 7 \mathrm{H} 25}(24), v_{\mathrm{C} 6 \mathrm{C} 7}(11), v_{\mathrm{C} 8 \mathrm{C} 19}(10), v_{\mathrm{C} 8 \mathrm{C} 16}(6), v_{\mathrm{C} 9 \mathrm{C} 16}(6), \rho_{\mathrm{N}_{3} \mathrm{H}_{23}}(5)$ \\
\hline 69 & 1469.8 & 18.3 & 1.1 & $\delta_{1 / \mathrm{C}_{20} \mathrm{H} 31 \mathrm{H} 32}(90)$ \\
\hline 70 & 1479.4 & 0.8 & 3.9 & $\rho_{\mathrm{C} 15 \mathrm{H} 29}(16), \rho_{\mathrm{C} 13 \mathrm{H} 27}(15), v_{\mathrm{C} 10 \mathrm{C} 14}(7), v_{\mathrm{C} 11 \mathrm{C} 12}(7), \rho_{\mathrm{C} 19 \mathrm{H} 30}(6)$, \\
\hline
\end{tabular}




\begin{tabular}{|c|c|c|c|c|}
\hline & & & & $v_{\mathrm{C} 8 \mathrm{C} 16}(5)$ \\
\hline 71 & 1489.1 & 20.0 & 0.9 & $\begin{array}{l}\rho_{\mathrm{C} 19 \mathrm{H} 30}(12), v_{\mathrm{C} 8 \mathrm{C} 16}(10), \rho_{\mathrm{C} 6 \mathrm{H} 24}(8), \rho_{\mathrm{C} 13 \mathrm{H} 27}(6), v_{\mathrm{C} 7 \mathrm{C} 18}(5), v_{\mathrm{N} 3 \mathrm{C} 18}(5), \\
v_{\mathrm{C} 8 \mathrm{C} 17}(5)\end{array}$ \\
\hline 72 & 1506.4 & 47.1 & 2.6 & $\delta_{1 / \mathrm{C}_{2} \mathrm{H}_{22} \mathrm{H}_{21}(73)}$ \\
\hline 73 & 1507.9 & 2.4 & 0.7 & $\delta_{1 / \mathrm{C} 2 \mathrm{H} 22 \mathrm{H} 21}(16), \rho_{\mathrm{C} 14 \mathrm{H} 28}(16), \rho_{\mathrm{C}_{12} \mathrm{H} 26}(15), v_{\mathrm{C} 10 \mathrm{C} 11}(14), v_{\mathrm{C} 13 \mathrm{C} 15}(6)$ \\
\hline 74 & 1550.9 & 215.7 & 1.0 & $v_{\mathrm{C} 6 \mathrm{C} 19}(18), \rho_{\mathrm{N} 3 \mathrm{H} 23}(18), v_{\mathrm{N} 3 \mathrm{C} 18}(16), \rho_{\mathrm{C} 6 \mathrm{H} 24}(12), v_{\mathrm{C} 6 \mathrm{C} 7}(7), v_{\mathrm{C} 8 \mathrm{C} 16}(6)$ \\
\hline 75 & 1610.3 & 41.1 & 12.5 & $\begin{array}{l}v_{\mathrm{C} 13 \mathrm{C} 15}(11), v_{\mathrm{C} 10 \mathrm{C} 11}(10), v_{\mathrm{O} 5 \mathrm{C} 9}(10), v_{\mathrm{C} 8 \mathrm{C} 19}(7), v_{\mathrm{C} 12 \mathrm{C} 13}(6), v_{\mathrm{C} 6 \mathrm{C} 19}(6), \\
v_{\mathrm{C} 16 \mathrm{C} 18}(5)\end{array}$ \\
\hline 76 & 1612.5 & 99.7 & 3.8 & $\begin{array}{l}\rho_{\mathrm{N} 3 \mathrm{H} 23}(20), v_{\mathrm{C} 6 \mathrm{C} 19}(8), v_{\mathrm{C} 16 \mathrm{C} 18}(7), v_{\mathrm{C} 13 \mathrm{C} 15}(7), v_{\mathrm{C} 10 \mathrm{C} 11}(6), v_{\mathrm{C} 7 \mathrm{C} 18}(6), \\
v_{\mathrm{C} 8 \mathrm{C} 19}(5)\end{array}$ \\
\hline 77 & 1625.7 & 36.2 & 18.2 & $v_{\mathrm{C}_{14} \mathrm{C} 15}(12), v_{\mathrm{O} 5 \mathrm{C} 9}(9), v_{\mathrm{C}_{11} \mathrm{C} 12}(9), v_{\mathrm{C} 6 \mathrm{C} 7}(8), v_{\mathrm{C} 8 \mathrm{C} 19}(6),{ }^{3} \beta_{2 / R 6}(6)$ \\
\hline 78 & 1630.9 & 252.5 & 6.2 & $v_{\mathrm{C} 6 \mathrm{C} 7}(12), v_{\mathrm{C} 14 \mathrm{C} 15}(9), v_{\mathrm{C} 12 \mathrm{C} 13}(8), v_{\mathrm{C}_{11} \mathrm{C} 12}(7), v_{\mathrm{C} 10 \mathrm{C} 14}(6), v_{\mathrm{O} 4 \mathrm{C} 17}(6)$ \\
\hline 79 & 1667.0 & 234.4 & 24.7 & $v_{\mathrm{O} 5 \mathrm{C} 9}(39),{ }^{2} \beta_{3 / R 6}(6),{ }^{2} \beta_{\text {trig/R6 }}(6)$ \\
\hline 80 & 1721.0 & 177.4 & 27.9 & $v_{\mathrm{O} 4 \mathrm{C} 17}(71),{ }^{2} \beta_{\text {trig/R6 }}(9)$ \\
\hline 81 & 2675.3 & 1.8 & 7.5 & $v_{\mathrm{S} 1 \mathrm{H} 33}(100)$ \\
\hline 82 & 2962.9 & 72.7 & 14.2 & $v_{\mathrm{C} 2 \mathrm{H} 22}(96)$ \\
\hline 83 & 3040.7 & 14.5 & 6.0 & $v_{\mathrm{C} 2 \mathrm{H} 21}(87), v_{\mathrm{C} 20 \mathrm{H} 32}(9)$ \\
\hline 84 & 3062.3 & 22.2 & 6.1 & $v_{\mathrm{C} 20 \mathrm{H} 32}(64), v_{\mathrm{C} 20 \mathrm{H} 31}(27), v_{\mathrm{C}_{2} \mathrm{H} 21}(8)$ \\
\hline 85 & 3112.6 & 10.0 & 5.9 & $v_{\mathrm{C}_{20} \mathrm{H} 31}(71), v_{\mathrm{C}_{20} \mathrm{H} 32}(27)$ \\
\hline 86 & 3170.1 & 3.3 & 4.5 & $v_{\mathrm{C} 15 \mathrm{H} 29}(49), v_{\mathrm{C} 13 \mathrm{H} 27}(45)$ \\
\hline 87 & 3172.8 & 11.8 & 8.1 & $v_{\mathrm{C} 6 \mathrm{H} 24}(90), v_{\mathrm{C} 7 \mathrm{H} 25}(6)$ \\
\hline 88 & 3184.8 & 13.2 & 14.5 & $v_{\mathrm{C} 13 \mathrm{H} 27}(43), v_{\mathrm{C} 15 \mathrm{H} 29}(42), v_{\mathrm{C} 12 \mathrm{H} 26}(9), v_{\mathrm{C} 14 \mathrm{H} 28}(5)$ \\
\hline 89 & 3201.7 & 3.7 & 6.9 & $v_{\mathrm{C} 12 \mathrm{H} 26}(82), v_{\mathrm{C} 13 \mathrm{H} 27}(9), v_{\mathrm{C} 14 \mathrm{H} 28}(9)$ \\
\hline 90 & 3205.9 & 9.5 & 11.0 & $v_{\mathrm{C} 14 \mathrm{H} 28}(83), v_{\mathrm{C} 15 \mathrm{H} 29}(8), v_{\mathrm{C} 12 \mathrm{H} 26}(6)$ \\
\hline 91 & 3206.8 & 6.5 & 8.6 & $v_{\mathrm{C} 7 \mathrm{H} 25}(87), v_{\mathrm{C} 19 \mathrm{H} 30}(9)$ \\
\hline 92 & 3212.4 & 4.7 & 14.0 & $v_{\mathrm{C} 19 \mathrm{H} 30}(87), v_{\mathrm{C} 7 \mathrm{H} 25}(7), v_{\mathrm{C} 6 \mathrm{H} 24}(5)$ \\
\hline 93 & 3447.9 & 78.0 & 30.4 & $v_{\mathrm{N} 3 \mathrm{H} 23}(99)$ \\
\hline
\end{tabular}


Table S.17. Frequencies of normal vibrations, IR and Raman intensities and PEDs for $T G G$ conformer calculated at the B3LYP/6-311++G(d,p) level of theory.

\begin{tabular}{|c|c|c|c|c|}
\hline $\mathbf{N r}$ & $\begin{array}{l}\text { Freq. } \\
{\left[\mathrm{cm}^{-1}\right]}\end{array}$ & IR Int. & \begin{tabular}{c|} 
Raman \\
Int.
\end{tabular} & PED $($ contributions $>5 \%)$ \\
\hline 1 & 23.2 & 1.4 & 13.3 & $\tau_{\mathrm{C} 2 \mathrm{~N} 3}(53),{ }^{1} \tau_{3 / R 6}(14),{ }^{2} \tau_{3 / R 6}(8), \tau_{\mathrm{C}_{2} \mathrm{C} 20}(6), \tau_{\mathrm{N} 3 \mathrm{C} 18}(5)$ \\
\hline 2 & 39.1 & 5.7 & 3.4 & ${ }^{2} \tau_{3 / R 6}(33),{ }^{2} \tau_{2 / R 6}(24), \tau_{\mathrm{N} 3 \mathrm{C} 18}(19)$ \\
\hline 3 & 48.3 & 0.4 & 1.5 & ${ }^{2} \tau_{3 / R 6}(29), \tau_{\mathrm{C}_{2} \mathrm{C} 20}(15), \omega_{\mathrm{N}_{3} \mathrm{H} 23}(12), \tau_{\mathrm{N} 3 \mathrm{C} 18}(9),{ }^{1} \tau_{3 / R 6}(7)$ \\
\hline 4 & 73.3 & 0.5 & 0.4 & $\begin{array}{l}{ }^{2} \tau_{2 / R 6}(12), \tau_{\mathrm{N} 3 \mathrm{C} 18}(11),{ }^{1} \tau_{3 / R 6}(10), \delta_{2 / \mathrm{C}_{2} \mathrm{H} 22 \mathrm{H} 21}(8),{ }^{1} \tau_{2 / R 6}(8), \\
{ }^{2} \tau_{3 / R 6}(8), \tau_{\mathrm{C}_{2 \mathrm{~N} 3}}(8)\end{array}$ \\
\hline 5 & 100.2 & 1.6 & 0.4 & $\omega_{\mathrm{N} 3 \mathrm{H} 23}(30),{ }_{2}^{2} \tau_{2 / R 6}(21), \beta_{\mathrm{C} 18 \mathrm{~N} 3 \mathrm{C} 2}(8), \rho_{\mathrm{C} 18 \mathrm{~N} 3}(7)$ \\
\hline 6 & 119.0 & 0.4 & 1.0 & ${ }^{2} \tau_{1 / R \sigma}(38),{ }^{3} \tau_{2 / R \sigma}(16),{ }^{2} \tau_{2 / R \sigma}(11),{ }^{3} \tau_{3 / R 6}(8)$ \\
\hline 7 & 144.6 & 0.8 & 0.8 & $\begin{array}{l}\omega_{\mathrm{N}_{3} \mathrm{H} 23}(20), \tau_{\mathrm{C}_{2} \mathrm{C} 20}(16),{ }^{2} \tau_{1 / R 6}(13), \delta_{2 / \mathrm{C} 20 \mathrm{H} 31 \mathrm{H} 32}(9), \tau_{\mathrm{N} 3 \mathrm{C} 18}(5), \\
{ }^{1} \tau_{2 / R 6}(5)\end{array}$ \\
\hline 8 & 165.4 & 0.6 & 0.0 & ${ }^{4} b f l_{R R}(25),{ }^{2} b f l_{R R}(23), \tau_{N 3 C l 8}(10),{ }^{2} \tau_{3 / R 6}(8), \omega_{C l 7 O 4}(6), \omega_{C 9 O 5}(6)$ \\
\hline 9 & 201.7 & 0.2 & 1.1 & ${ }^{1} \tau_{3 / R 6}(26),{ }^{1} \tau_{2 / R 6}(22),{ }^{4} b f l_{R R}(16), \omega_{C l 9 H 30}(7)$ \\
\hline 10 & 215.0 & 6.7 & 0.2 & $\tau_{\mathrm{S} 1 \mathrm{C} 20_{2}}(40), \delta_{2 / \mathrm{C}_{2} \mathrm{H} 22 \mathrm{H} 21}(16), \tau_{\mathrm{C}_{2} \mathrm{C} 20}(9), \delta_{2 / \mathrm{C}_{20} \mathrm{H} 31 \mathrm{H} 32}(5)$ \\
\hline 11 & 249.2 & 1.3 & 0.2 & $\beta_{\mathrm{C}_{18 \mathrm{~N} 3 \mathrm{C} 2}}(17), \delta_{2 / \mathrm{C} 20 \mathrm{H} 31 \mathrm{H} 32}(15),{ }^{3} \beta_{2 / R 6}(10), v_{\mathrm{C} 9 \mathrm{C} 10}(5), \tau_{\mathrm{S}_{\mathrm{C} 20}}(5)$ \\
\hline 12 & 257.3 & 13.8 & 0.5 & $\tau_{\mathrm{S} 1 \mathrm{C} 20}(36), \tau_{\mathrm{C}_{2} \mathrm{C} 20}(11),{ }^{2} b f l_{R R}(8), \delta_{2 / \mathrm{C}_{2} \mathrm{OH} 31 \mathrm{H} 32}(7)$ \\
\hline 13 & 273.8 & 5.7 & 0.6 & ${ }^{2} b f l_{R R}(23), \omega_{N 3 H 23}(11), \tau_{C 2 C 20}(7), \omega_{C 18 N 3}(7),{ }^{1} \tau_{2 / R 6}(7)$ \\
\hline 14 & 310.1 & 0.9 & 0.3 & ${ }^{2} \beta_{2 / R 6}(35), \beta_{\mathrm{C} 18 \mathrm{~N} 3 \mathrm{C} 2_{2}}(9), \delta_{2 / \mathrm{C} 20 \mathrm{H} 31 \mathrm{H} 32}(9), v_{\mathrm{C} 8 \mathrm{C} 17}(5)$ \\
\hline 15 & 343.5 & 1.2 & 0.3 & ${ }^{2} \beta_{3 / R 6}(28), \rho_{\mathrm{C} 17 \mathrm{O} 4}(20), v_{\mathrm{C} 8 \mathrm{C} 17}(8), \rho_{\mathrm{C} 18 \mathrm{~N} 3}(8)$ \\
\hline 16 & 369.5 & 8.3 & 1.5 & 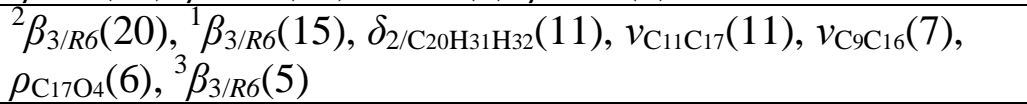 \\
\hline 17 & 424.0 & 21.6 & 0.5 & $\rho_{\mathrm{C} 9 \mathrm{O} 5}(39), \rho_{\mathrm{C} 17 \mathrm{O} 4}(18),{ }^{1} \beta_{3 / R 6}(9), v_{\mathrm{C} 9 \mathrm{C} 16}(9)$ \\
\hline 18 & 427.3 & 0.6 & 0.0 & ${ }^{3} \tau_{3 / R 6}(59),{ }^{4} b f l_{R R}(15),{ }^{3} \tau_{2 / R 6}(7)$ \\
\hline 19 & 433.2 & 0.1 & 0.2 & ${ }^{3} \tau_{2 / R \sigma}(26),{ }^{1} \tau_{2 / R 6}(19),{ }^{1} \tau_{3 / R 6}(14),{ }^{2} \tau_{1 / R 6}(6), \omega_{\mathrm{C} 9 \mathrm{O} 5}(5)$ \\
\hline 20 & 437.7 & 0.9 & 0.4 & $\begin{array}{l}\delta_{2 / \mathrm{C} 2 \mathrm{H} 22 \mathrm{H} 21}(17), \delta_{2 / \mathrm{C}_{20} \mathrm{H} 31 \mathrm{H} 32}(15),{ }^{2} \beta_{3 / R 6}(11), \rho_{\mathrm{C} 18 \mathrm{~N} 3}(9),{ }^{3} \tau_{2 / R 6}(7), \\
{ }^{1} \beta_{2 / R 6}(6)\end{array}$ \\
\hline 21 & 472.1 & 2.6 & 1.4 & ${ }^{2} \beta_{2 / R \sigma}(34), \rho_{\mathrm{C} 18 \mathrm{~N} 3}(12),{ }^{1} \beta_{2 / R \sigma}(11),{ }^{3} \beta_{2 / R \sigma}(7),{ }^{3} \beta_{3 / R 6}(6)$ \\
\hline 22 & 486.8 & 0.6 & 9.7 & ${ }^{2} \beta_{3 / R \sigma}(26),{ }^{1} \beta_{3 / R \sigma}(8), \delta_{2 / \mathrm{C} 2 \mathrm{H} 22 \mathrm{H} 21}(7), \rho_{\mathrm{C} 18 \mathrm{~N} 3}(7),{ }^{1} \beta_{2 / R \sigma}(6),{ }^{3} \beta_{2 / R 6}(6)$ \\
\hline 23 & 491.6 & 1.9 & 0.1 & ${ }^{3} \tau_{2 / R \sigma}(23),{ }^{1} \tau_{3 / R \sigma}(23),{ }^{1} \tau_{2 / R 6}(11),{ }^{2} \tau_{2 / R 6}(9),{ }^{3} \tau_{3 / R \sigma}(5),{ }^{1} \tau_{1 / R 6}(5)$ \\
\hline 24 & 558.4 & 7.2 & 0.1 & $\omega_{\mathrm{C} 18 \mathrm{~N} 3}(29),{ }^{1} \tau_{3 / R \sigma}(14),{ }^{1} \tau_{2 / R 6}(13),{ }^{1} \tau_{1 / R 6}(11),{ }^{2} b f l_{R R}(6)$ \\
\hline 25 & 615.8 & 6.9 & 0.9 & ${ }^{1} \beta_{2 / R \sigma}(21),{ }^{2} \beta_{\text {trig/R6}}(20),{ }^{3} \beta_{2 / R 6}(15),{ }^{1} \beta_{3 / R 6}(9)$ \\
\hline 26 & 636.1 & 2.9 & 1.2 & ${ }^{2} \beta_{\text {trig/R6 }}(26), v_{\mathrm{S} 1 \mathrm{C} 20}(12),{ }^{1} \beta_{3 / R 6}(10),{ }^{3} \beta_{3 / R 6}(9),{ }^{1} \beta_{2 / R 6}(5)$ \\
\hline 27 & 647.5 & 20.3 & 1.3 & $v_{\mathrm{S}_{1} \mathrm{C} 20}(30), \tau_{\mathrm{N} 3 \mathrm{C} 18}(23), \omega_{\mathrm{N} 3 \mathrm{H} 23}(17), \tau_{\mathrm{C}_{2 \mathrm{~N}} 3}(6)$ \\
\hline 28 & 659.3 & 16.6 & 0.6 & $v_{\mathrm{S} 1 \mathrm{C} 20}(29),{ }^{3} \beta_{3 / R 6}(17), \tau_{\mathrm{N} 3 \mathrm{C} 18}(7), \omega_{\mathrm{N} 3 \mathrm{H} 23}(7),{ }^{3} \beta_{2 / R 6}(5)$ \\
\hline 29 & 673.7 & 9.2 & 0.1 & ${ }^{3} \tau_{1 / R 6}(38),{ }^{1} \tau_{1 / R 6}(18), \omega_{\mathrm{C} 9 \mathrm{O} 5}(12), \omega_{\mathrm{C} 18 \mathrm{~N} 3}(10)$ \\
\hline 30 & 689.5 & 8.4 & 1.9 & ${ }^{3} \beta_{3 / R 6}(25),{ }_{3 / R 6}(15), \beta_{\mathrm{C} 18 \mathrm{~N}_{3} \mathrm{C} 2}(7), v_{\mathrm{S}_{1 \mathrm{C}} 20}(6), \rho_{\mathrm{C} 9 \mathrm{O} 5}(6), \rho_{\mathrm{C} 17 \mathrm{O} 4}(6)$ \\
\hline 31 & 710.9 & 4.9 & 0.5 & ${ }^{3} \beta_{2 / R 6}(23), \rho_{\mathrm{C} 17 \mathrm{O} 4}(15), \rho_{\mathrm{C} 9 \mathrm{O} 5}(14),{ }^{1} \beta_{2 / R 6}(11), \rho_{\mathrm{C} 18 \mathrm{~N}_{3}}(6)$ \\
\hline 32 & 724.2 & 35.1 & 0.0 & ${ }^{3} \tau_{1 / R 6}(29),{ }^{1} \tau_{1 / R 6}(26), \omega_{\mathrm{C} 6 \mathrm{H} 24}(9), \omega_{\mathrm{C} 15 \mathrm{H} 29}(7), \omega_{\mathrm{C} 17 \mathrm{O} 4}(6)$ \\
\hline 33 & 747.0 & 54.6 & 0.0 & $\begin{array}{l}\omega_{\mathrm{C} 13 \mathrm{H} 27}(14),{ }^{1} \tau_{1 / R 6}(14), \omega_{\mathrm{C} 18 \mathrm{~N} 3}(13), \omega_{\mathrm{C} 17 \mathrm{O} 4}(9), \omega_{\mathrm{C} 9 \mathrm{O} 5}(9), \\
\omega_{\mathrm{C} 15 \mathrm{H} 29}(7), \omega_{\mathrm{C} 14 \mathrm{H} 28}(7),{ }^{3} \tau_{1 / R 6}(6)\end{array}$ \\
\hline 34 & 778.7 & 1.8 & 1.4 & $\rho_{\mathrm{C}_{20 \mathrm{H} 31 \mathrm{H} 32}}(36), \beta_{\mathrm{C} 20 \mathrm{~S} 1 \mathrm{H} 33}(17),{ }^{1} \beta_{3 / R 6}(8)$ \\
\hline 35 & 784.8 & 9.5 & 0.1 & $\begin{array}{l}{ }^{3} \tau_{1 / R 6}(19), \omega_{\mathrm{C} 7 \mathrm{H} 25}(14),{ }^{2} \tau_{1 / R 6}(13), \omega_{\mathrm{C} 19 \mathrm{H} 30}(11), \omega_{\mathrm{C} 6 \mathrm{H} 24}(10), \\
\omega_{\mathrm{C} 9 \mathrm{O} 5}(10), \omega_{\mathrm{C} 17 \mathrm{O} 4}(9)\end{array}$ \\
\hline 36 & 805.0 & 0.7 & 0.2 & $\begin{array}{l}{ }^{1} \beta_{\text {trig/R6 }}(14),{ }^{3} \beta_{\text {trig/R6 }}(8), \rho_{\mathrm{C} 20 \mathrm{H} 31 \mathrm{H} 32}(8),{ }^{3} \beta_{2 / R 6}(8),{ }^{2} \beta_{\text {trig/R6 }}(8), \\
{ }^{1} \beta_{2 / R 6}(8), \beta_{\mathrm{C} 20 \mathrm{~S} 1 \mathrm{H} 33}(8),{ }^{1} \beta_{3 / R 6}(5)\end{array}$ \\
\hline 37 & 810.7 & 1.3 & 0.1 & $\begin{array}{l}\omega_{\mathrm{C} 9 \mathrm{O} 5}(26), \omega_{\mathrm{C} 13 \mathrm{H} 27}(14),{ }^{1} \tau_{1 / R 6}(11), \omega_{\mathrm{C} 18 \mathrm{~N} 3}(9), \omega_{\mathrm{C} 15 \mathrm{H} 29}(8), \\
{ }^{2} \tau_{1 / R 6}(6)\end{array}$ \\
\hline
\end{tabular}




\begin{tabular}{|c|c|c|c|c|}
\hline 38 & 827.1 & 28.1 & 0.0 & $\begin{array}{l}\omega_{\mathrm{C} 7 \mathrm{H} 25}(22),{ }^{1} \tau_{1 / R 6}(19), \omega_{\mathrm{C} 17 \mathrm{O} 4}(17), \omega_{\mathrm{C} 18 \mathrm{~N} 3}(10),{ }^{2} \tau_{1 / R 6}(8), \\
\omega_{\mathrm{C} 15 \mathrm{H} 29}(5)\end{array}$ \\
\hline 39 & 880.7 & 20.6 & 1.7 & $\beta_{\mathrm{C}_{20 \mathrm{~S} 1 \mathrm{H} 33}}(25), \rho_{\mathrm{C} 2 \mathrm{H} 22 \mathrm{H} 21}(9), \tau_{\mathrm{C} 20 \mathrm{H} 31 \mathrm{H} 32}(8), v_{\mathrm{C} 7 \mathrm{C} 18}(6)$ \\
\hline 40 & 907.9 & 2.1 & 0.1 & $\omega_{\mathrm{C} 19 \mathrm{H} 30}(39), \omega_{\mathrm{C} 7 \mathrm{H} 25}(27), \omega_{\mathrm{C}_{12} \mathrm{H} 26}(6), \omega_{\mathrm{C} 14 \mathrm{H} 28}(6)$ \\
\hline 41 & 916.1 & 0.9 & 0.0 & $\omega_{\mathrm{C} 14 \mathrm{H} 28}(25), \omega_{\mathrm{C} 12 \mathrm{H} 26}(24), \omega_{\mathrm{C} 13 \mathrm{H} 27}(12), \omega_{\mathrm{C} 15 \mathrm{H} 29}(10), \omega_{\mathrm{C} 19 \mathrm{H} 30}(7)$ \\
\hline 42 & 922.0 & 17.7 & 0.7 & $\begin{array}{l}\beta_{\mathrm{C}_{20 \mathrm{~S} 1 \mathrm{H} 33}}(16), \rho_{\mathrm{C} 2 \mathrm{H} 22 \mathrm{H} 21}(9), v_{\mathrm{C}_{2} \mathrm{C} 20}(7), \rho_{\mathrm{C}_{20} \mathrm{H}_{31 \mathrm{H} 32}}(7), v_{\mathrm{C} 16 \mathrm{C} 18}(6), \\
\rho_{\mathrm{C}_{17 \mathrm{O} 4}}(5)\end{array}$ \\
\hline 43 & 928.9 & 1.8 & 1.1 & ${ }^{1} \beta_{\text {trig/R6 }}(26),{ }^{3} \beta_{\text {trig/R6}}(24), \rho_{\mathrm{C} 9 \mathrm{O} 5}(13), \rho_{\mathrm{C} 17 \mathrm{O} 4}(6), v_{\mathrm{C} 16 \mathrm{C} 18}(6)$ \\
\hline 44 & 989.9 & 17.0 & 0.3 & $v_{\mathrm{C}_{2} \mathrm{C} 20}(32), \rho_{\mathrm{C} 2 \mathrm{H} 22 \mathrm{H} 21}(24), \delta_{2 / \mathrm{C} 2 \mathrm{H} 22 \mathrm{H} 21}(8), \beta_{\mathrm{C} 18 \mathrm{~N} 3 \mathrm{C} 2_{2}}(6), v_{\mathrm{S}_{1} \mathrm{C} 20}(6)$ \\
\hline 45 & 998.9 & 0.9 & 0.1 & $\omega_{\mathrm{C} 6 \mathrm{H} 24}(49), \omega_{\mathrm{C} 19 \mathrm{H} 30}(21), \omega_{\mathrm{C} 7 \mathrm{H} 25}(10),{ }^{1} \tau_{1 / R 6}(7)$ \\
\hline 46 & 1002.6 & 2.0 & 0.0 & $\omega_{\mathrm{C} 12 \mathrm{H} 26}(34), \omega_{\mathrm{C} 14 \mathrm{H} 28}(27), \omega_{\mathrm{C} 13 \mathrm{H} 27}(13), \omega_{\mathrm{C} 15 \mathrm{H} 29}(8)$ \\
\hline 47 & 1016.4 & 0.0 & 0.0 & $\omega_{\mathrm{C} 15 \mathrm{H} 29}(32), \omega_{\mathrm{C} 13 \mathrm{H} 27}(26), \omega_{\mathrm{C} 14 \mathrm{H} 28}(18),{ }^{3} \tau_{1 / R 6}(12), \omega_{\mathrm{C}_{12 \mathrm{H} 26}}(11)$ \\
\hline 48 & 1023.3 & 76.8 & 3.3 & $v_{\mathrm{C} 8 \mathrm{C} 17}(15), v_{\mathrm{C}_{2} \mathrm{C} 20}(9), v_{\mathrm{C} 13 \mathrm{C} 15}(8), v_{\mathrm{C}_{11} \mathrm{C}_{12}}(7), v_{\mathrm{C} 12 \mathrm{C} 13}(6), v_{\mathrm{N} 3 \mathrm{C} 18}(5)$ \\
\hline 49 & 1058.8 & 0.8 & 4.7 & $v_{\mathrm{C} 13 \mathrm{C} 15}(31), \rho_{\mathrm{C} 12 \mathrm{H} 26}(12), v_{\mathrm{C} 14 \mathrm{C} 15}(12), \rho_{\mathrm{C} 14 \mathrm{H} 28}(11), v_{\mathrm{C} 12 \mathrm{C} 13}(9)$ \\
\hline 50 & 1096.1 & 13.8 & 1.1 & 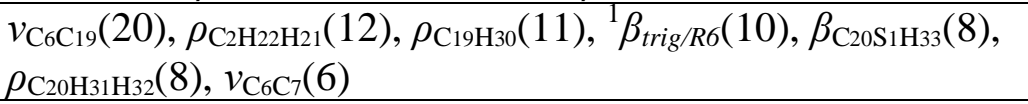 \\
\hline 51 & 1100.9 & 7.4 & 1.0 & $\begin{array}{l}\rho_{\mathrm{C} 2 \mathrm{H} 22 \mathrm{H} 21}(23), v_{\mathrm{C}_{2} \mathrm{C} 20}(13), v_{\mathrm{C} 6 \mathrm{C} 19}(12), \beta_{\mathrm{C}_{20 \mathrm{~S} 1 \mathrm{H} 33}}(11), \rho_{\mathrm{C} 20 \mathrm{H} 31 \mathrm{H} 32}(9), \\
\rho_{\mathrm{C} 19 \mathrm{H} 30}(6)\end{array}$ \\
\hline 52 & 1113.0 & 1.1 & 0.3 & $\begin{array}{l}{ }^{3} \beta_{\text {trig/R6 }}(38), v_{\mathrm{C} 12 \mathrm{C} 13}(8), v_{\mathrm{C} 14 \mathrm{C} 15}(8), \rho_{\mathrm{C} 13 \mathrm{H} 27}(8), \rho_{\mathrm{C} 15 \mathrm{H} 29}(7), \\
v_{\mathrm{C} 9 \mathrm{C} 10}(6), \rho_{\mathrm{C} 14 \mathrm{H} 28}(5), v_{\mathrm{C} 11 \mathrm{C} 17}(5)\end{array}$ \\
\hline 53 & 1140.3 & 3.9 & 1.8 & $v_{\mathrm{C}_{2} \mathrm{~N} 3}(44), v_{\mathrm{C}_{2} \mathrm{C} 20}(10), \tau_{\mathrm{C}_{20} \mathrm{H} 31 \mathrm{H} 32}(9), \rho_{\mathrm{C} 18 \mathrm{~N} 3}(6)$ \\
\hline 54 & 1176.0 & 3.9 & 4.8 & $\begin{array}{l}\rho_{\mathrm{C} 13 \mathrm{H} 27}(17), \rho_{\mathrm{C} 12 \mathrm{H} 26}(13), v_{\mathrm{C} 9 \mathrm{C} 16}(9), v_{\mathrm{C} 9 \mathrm{C} 10}(7),{ }^{1} \beta_{\text {trig/R6}}(6), \\
{ }^{3} \beta_{\text {trig/R6 }}(6)\end{array}$ \\
\hline 55 & 1183.9 & 16.9 & 0.5 & $\begin{array}{l}\rho_{\mathrm{C} 15 \mathrm{H} 29}(31), \rho_{\mathrm{C} 14 \mathrm{H} 28}(10), v_{\mathrm{C} 14 \mathrm{C} 15}(9), \rho_{\mathrm{C} 13 \mathrm{H} 27}(7),{ }^{1} \beta_{\text {trig/R6 }}(6), \\
{ }_{\text {trig/R6}}(6), v_{\mathrm{C} 13 \mathrm{C} 15}(5)\end{array}$ \\
\hline 56 & 1197.6 & 28.2 & 8.6 & $\rho_{\mathrm{C} 6 \mathrm{H} 24}(31), \rho_{\mathrm{C} 7 \mathrm{H} 25}(14), v_{\mathrm{C} 6 \mathrm{C} 7}(8), \rho_{\mathrm{C} 13 \mathrm{H} 27}(6)$ \\
\hline 57 & 1213.8 & 1.9 & 5.3 & $\begin{array}{l}\rho_{\mathrm{C} 19 \mathrm{H} 30}(24), \rho_{\mathrm{C} 7 \mathrm{H} 25}(10), v_{\mathrm{C} 8 \mathrm{C} 19}(10), v_{\mathrm{C} 11 \mathrm{C} 17}(8), \rho_{\mathrm{C} 14 \mathrm{H} 28}(6), \\
\rho_{\mathrm{C} 6 \mathrm{H} 24}(6)\end{array}$ \\
\hline 58 & 1236.4 & 0.3 & 0.3 & $\tau_{\mathrm{C} 20 \mathrm{H} 31 \mathrm{H} 32}(41), \tau_{\mathrm{C}_{2} \mathrm{H} 22 \mathrm{H} 21}(28), v_{\mathrm{C}_{2} \mathrm{~N} 3}(5)$ \\
\hline 59 & 1250.8 & 111.0 & 3.4 & $\tau_{\mathrm{C}_{2} \mathrm{H} 22 \mathrm{H} 21}(19), \omega_{\mathrm{C} 20 \mathrm{H} 31 \mathrm{H} 32}(10), v_{\mathrm{C} 7 \mathrm{C} 18}(10), \rho_{\mathrm{C} 12 \mathrm{H} 26}(9), v_{\mathrm{C} 9 \mathrm{C} 10}(6)$ \\
\hline 60 & 1275.5 & 31.7 & 0.4 & $\begin{array}{l}\tau_{\mathrm{C} 20 \mathrm{H} 31 \mathrm{H} 32}(12), \tau_{\mathrm{C}_{2} \mathrm{H} 22 \mathrm{H} 21}(11), \omega_{\mathrm{C} 20 \mathrm{H} 31 \mathrm{H} 32}(10),{ }^{2} \beta_{\text {trig/R6}}(10), \\
\rho_{\mathrm{C} 14 \mathrm{H} 28}(10), \rho_{\mathrm{C} 12 \mathrm{H} 26}(9)\end{array}$ \\
\hline 61 & 1292.7 & 515.4 & 9.1 & $\begin{array}{l}v_{\mathrm{C} 9 \mathrm{C} 10}(12), v_{\mathrm{C} 9 \mathrm{C} 16}(8), v_{\mathrm{C} 8 \mathrm{C} 16}(7), v_{\mathrm{C} 7 \mathrm{C} 18}(6), \rho_{\mathrm{C} 14 \mathrm{H} 28}(5), v_{\mathrm{C} 8 \mathrm{C} 17}(5), \\
v_{\mathrm{C}_{11} \mathrm{C} 17}(5)\end{array}$ \\
\hline 62 & 1316.0 & 175.3 & 1.4 & $v_{\mathrm{C} 11 \mathrm{C} 17}(18), v_{\mathrm{C} 8 \mathrm{C} 17}(14), \rho_{\mathrm{C} 12 \mathrm{H} 26}(8), \rho_{\mathrm{C} 17 \mathrm{O} 4}(7), v_{\mathrm{C} 16 \mathrm{C} 18}(5),{ }^{2} \beta_{2 / R 6}(5)$ \\
\hline 63 & 1327.1 & 69.6 & 3.5 & $\begin{array}{l}\omega_{\mathrm{C} 20 \mathrm{H} 31 \mathrm{H} 32}(16), v_{\mathrm{N} 3 \mathrm{C} 18}(14), \rho_{\mathrm{N} 3 \mathrm{H} 23}(11), \omega_{\mathrm{C} 2 \mathrm{H} 22 \mathrm{H} 21}(9), \rho_{\mathrm{C} 6 \mathrm{H} 24}(7), \\
v_{\mathrm{C} 16 \mathrm{C} 18}(5)\end{array}$ \\
\hline 64 & 1338.9 & 52.4 & 1.5 & $\omega_{\mathrm{C} 20 \mathrm{H} 31 \mathrm{H} 32}(44), \tau_{\mathrm{C} 2 \mathrm{H} 22 \mathrm{H} 21}(18), v_{\mathrm{N} 3 \mathrm{C} 18}(6)$ \\
\hline 65 & 1358.5 & 14.9 & 0.8 & $\begin{array}{l}v_{\mathrm{C}_{10 \mathrm{C} 14}}(15), v_{\mathrm{C} 10 \mathrm{C} 11}(15), v_{\mathrm{C} 11 \mathrm{C} 12}(14), v_{\mathrm{C} 13 \mathrm{C} 15}(14), v_{\mathrm{C} 12 \mathrm{C} 13}(13), \\
v_{\mathrm{C}_{14 \mathrm{C} 15}}(13)\end{array}$ \\
\hline 66 & 1374.9 & 9.3 & 3.2 & $\begin{array}{l}v_{\mathrm{C}_{16} \mathrm{C} 18}(13), v_{\mathrm{C} 8 \mathrm{C} 16}(12), v_{\mathrm{C} 8 \mathrm{C} 19}(10), v_{\mathrm{C} 9 \mathrm{C} 16}(8), v_{\mathrm{C} 6 \mathrm{C} 19}(7), v_{\mathrm{C} 9 \mathrm{C} 10}(6), \\
v_{\mathrm{C} 7 \mathrm{C} 18}(6), \rho_{\mathrm{C} 9 \mathrm{O} 5}(6)\end{array}$ \\
\hline 67 & 1406.3 & 12.5 & 1.5 & $\omega_{\mathrm{C} 2 \mathrm{H} 22 \mathrm{H} 21}(57), \rho_{\mathrm{C} 7 \mathrm{H} 25}(5)$ \\
\hline 68 & 1434.2 & 65.4 & 0.2 & $\begin{array}{l}\rho_{\mathrm{C} 7 \mathrm{H} 25}(22), v_{\mathrm{C} 6 \mathrm{C} 7}(10), v_{\mathrm{C} 8 \mathrm{C} 19}(10), v_{\mathrm{C} 8 \mathrm{C} 16}(7), v_{\mathrm{C} 9 \mathrm{C} 16}(7), \rho_{\mathrm{N} 3 \mathrm{H} 23}(6), \\
\omega_{\mathrm{C} 2 \mathrm{H} 22 \mathrm{H} 21}(6)\end{array}$ \\
\hline 69 & 1467.6 & 13.5 & 0.9 & $\delta_{1 / \mathrm{C}_{20} \mathrm{H} 31 \mathrm{H} 32}(92)$ \\
\hline 70 & 1479.6 & 0.5 & 3.1 & $\rho_{\mathrm{C} 15 \mathrm{H} 29}(17), \rho_{\mathrm{C} 13 \mathrm{H} 27}(15), v_{\mathrm{C} 10 \mathrm{C} 14}(8), v_{\mathrm{C} 11 \mathrm{C} 12}(7), \rho_{\mathrm{C} 19 \mathrm{H} 30}(5)$ \\
\hline 71 & 1489.3 & 12.4 & 0.9 & $\rho_{\mathrm{C} 19 \mathrm{H} 30}(13), v_{\mathrm{C} 8 \mathrm{C} 16}(11), \rho_{\mathrm{C} 6 \mathrm{H} 24}(9), v_{\mathrm{C} 7 \mathrm{C} 18}(6), \rho_{\mathrm{C} 13 \mathrm{H} 27}(6), v_{\mathrm{C} 8 \mathrm{C} 17}(5)$ \\
\hline 72 & 1507.6 & 10.2 & 2.0 & $\rho_{\mathrm{C} 14 \mathrm{H} 28}(20), \rho_{\mathrm{C} 12 \mathrm{H} 26}(19), v_{\mathrm{C} 10 \mathrm{C} 11}(16), v_{\mathrm{C} 13 \mathrm{C} 15}(8), \rho_{\mathrm{C} 15 \mathrm{H} 29}(5)$ \\
\hline
\end{tabular}




\begin{tabular}{|c|c|c|c|c|}
\hline 73 & 1514.4 & 58.1 & 0.9 & $\delta_{1 / \mathrm{C}_{2} \mathrm{H}_{22 \mathrm{H} 21}(88)}$ \\
\hline 74 & 1547.8 & 260.3 & 0.6 & $\rho_{\mathrm{N} 3 \mathrm{H} 23}(22), v_{\mathrm{N} 3 \mathrm{C} 18}(18), v_{\mathrm{C} 6 \mathrm{C} 19}(16), \rho_{\mathrm{C} 6 \mathrm{H} 24}(10), v_{\mathrm{C} 8 \mathrm{C} 16}(6), v_{\mathrm{C} 6 \mathrm{C} 7}(5)$ \\
\hline 75 & 1606.5 & 133.6 & 7.4 & $\begin{array}{l}\rho_{\mathrm{N}_{3 \mathrm{H} 23}(}(19), v_{\mathrm{C} 6 \mathrm{C} 19}(15), v_{\mathrm{C} 16 \mathrm{C} 18}(12),{ }^{1} \beta_{2 / R 6}(9), v_{\mathrm{C} 8 \mathrm{C} 19}(9), v_{\mathrm{C} 7 \mathrm{C} 18}(7), \\
\rho_{\mathrm{C} 18 \mathrm{~N} 3}(6)\end{array}$ \\
\hline 76 & 1612.9 & 19.1 & 2.2 & $\begin{array}{l}v_{\mathrm{C}_{13 \mathrm{C} 15}}(21), v_{\mathrm{C} 10 \mathrm{C} 11}(21), v_{\mathrm{O} 5 \mathrm{C} 9}(10), v_{\mathrm{C} 12 \mathrm{C} 13}(10),{ }^{3} \beta_{3 / R 6}(9), \\
v_{\mathrm{C} 10 \mathrm{C} 14}(8), \rho_{\mathrm{C} 13 \mathrm{H} 27}(7)\end{array}$ \\
\hline 77 & 1626.2 & 47.6 & 18.1 & $v_{\mathrm{C} 6 \mathrm{C} 7}(12), v_{\mathrm{C} 8 \mathrm{C} 19}(10), v_{\mathrm{O} 5 \mathrm{C} 9}(10), v_{\mathrm{C}_{14} \mathrm{C}_{15}}(8), v_{\mathrm{C}_{11 \mathrm{C} 12}}(6),{ }^{1} \beta_{3 / R 6}(5)$ \\
\hline 78 & 1630.3 & 235.2 & 6.7 & $\begin{array}{l}v_{\mathrm{C} 14 \mathrm{C} 15}(12), v_{\mathrm{C} 6 \mathrm{C} 7}(9), v_{\mathrm{C} 11 \mathrm{C} 12}(9), v_{\mathrm{C} 12 \mathrm{C} 13}(9), v_{\mathrm{C} 10 \mathrm{C} 14}(7), v_{\mathrm{O} 4 \mathrm{C} 17}(6), \\
{ }^{3} \beta_{2 / R 6}(6)\end{array}$ \\
\hline 79 & 1673.6 & 209.3 & 29.6 & $v_{\mathrm{O} 5 \mathrm{C} 9}(47),{ }^{2} \beta_{\text {trig/R6 }}(6),{ }^{2} \beta_{3 / R 6}(6)$ \\
\hline 80 & 1720.3 & 185.3 & 27.4 & $v_{\mathrm{O} 4 \mathrm{C} 17}(71),{ }^{2} \beta_{\text {trig/R6 }}(9)$ \\
\hline 81 & 2661.6 & 4.6 & 7.8 & $v_{\mathrm{S}_{1 \mathrm{H} 33}}(100)$ \\
\hline 82 & 2969.3 & 78.4 & 11.9 & $v_{\mathrm{C}_{2} \mathrm{H} 21}(88), v_{\mathrm{C}_{2} \mathrm{H} 22}(11)$ \\
\hline 83 & 3004.7 & 24.5 & 5.6 & $v_{\mathrm{C}_{2} \mathrm{H} 22}(87), v_{\mathrm{C} 2 \mathrm{H} 21}(11)$ \\
\hline 84 & 3065.7 & 20.0 & 8.1 & $v_{\mathrm{C}_{20 \mathrm{H} 32}}(55), v_{\mathrm{C}_{20 \mathrm{H} 31}}(44)$ \\
\hline 85 & 3113.5 & 10.0 & 4.9 & $v_{\mathrm{C}_{20 \mathrm{H} 31}}(55), v_{\mathrm{C}_{20 \mathrm{H} 32}}(44)$ \\
\hline 86 & 3169.7 & 3.5 & 4.5 & $v_{\mathrm{C} 15 \mathrm{H} 29}(48), v_{\mathrm{C} 13 \mathrm{H} 27}(46)$ \\
\hline 87 & 3171.6 & 12.8 & 8.1 & $v_{\mathrm{C} 6 \mathrm{H} 24}(90), v_{\mathrm{C} 7 \mathrm{H} 25}(6)$ \\
\hline 88 & 3184.5 & 13.8 & 14.4 & $v_{\mathrm{C} 15 \mathrm{H} 29}(43), v_{\mathrm{C} 13 \mathrm{H} 27}(42), v_{\mathrm{C} 12 \mathrm{H} 26}(9), v_{\mathrm{C} 14 \mathrm{H} 28}(5)$ \\
\hline 89 & 3201.5 & 3.7 & 6.7 & $v_{\mathrm{C}_{12} \mathrm{H} 26}(81), v_{\mathrm{C} 14 \mathrm{H} 28}(9), v_{\mathrm{C} 13 \mathrm{H} 27}(9)$ \\
\hline 90 & 3205.3 & 11.2 & 6.6 & $v_{\mathrm{C} 7 \mathrm{H} 25}(88), v_{\mathrm{C} 19 \mathrm{H} 30}(5)$ \\
\hline 91 & 3205.7 & 7.4 & 13.7 & $v_{\mathrm{C}_{14 \mathrm{H} 28}}(82), v_{\mathrm{C} 15 \mathrm{H} 29}(8), v_{\mathrm{C} 12 \mathrm{H} 26}(6)$ \\
\hline 92 & 3212.6 & 4.4 & 13.1 & $v_{\mathrm{C} 19 \mathrm{H} 30}(91)$ \\
\hline 93 & 3453.4 & 76.2 & 33.0 & $v_{\mathrm{N} 3 \mathrm{H} 23}(99)$ \\
\hline
\end{tabular}




$$
\mathrm{S}_{\mathrm{VIB}}=-0.08 v+17.5(\mathrm{R}=0.923)
$$

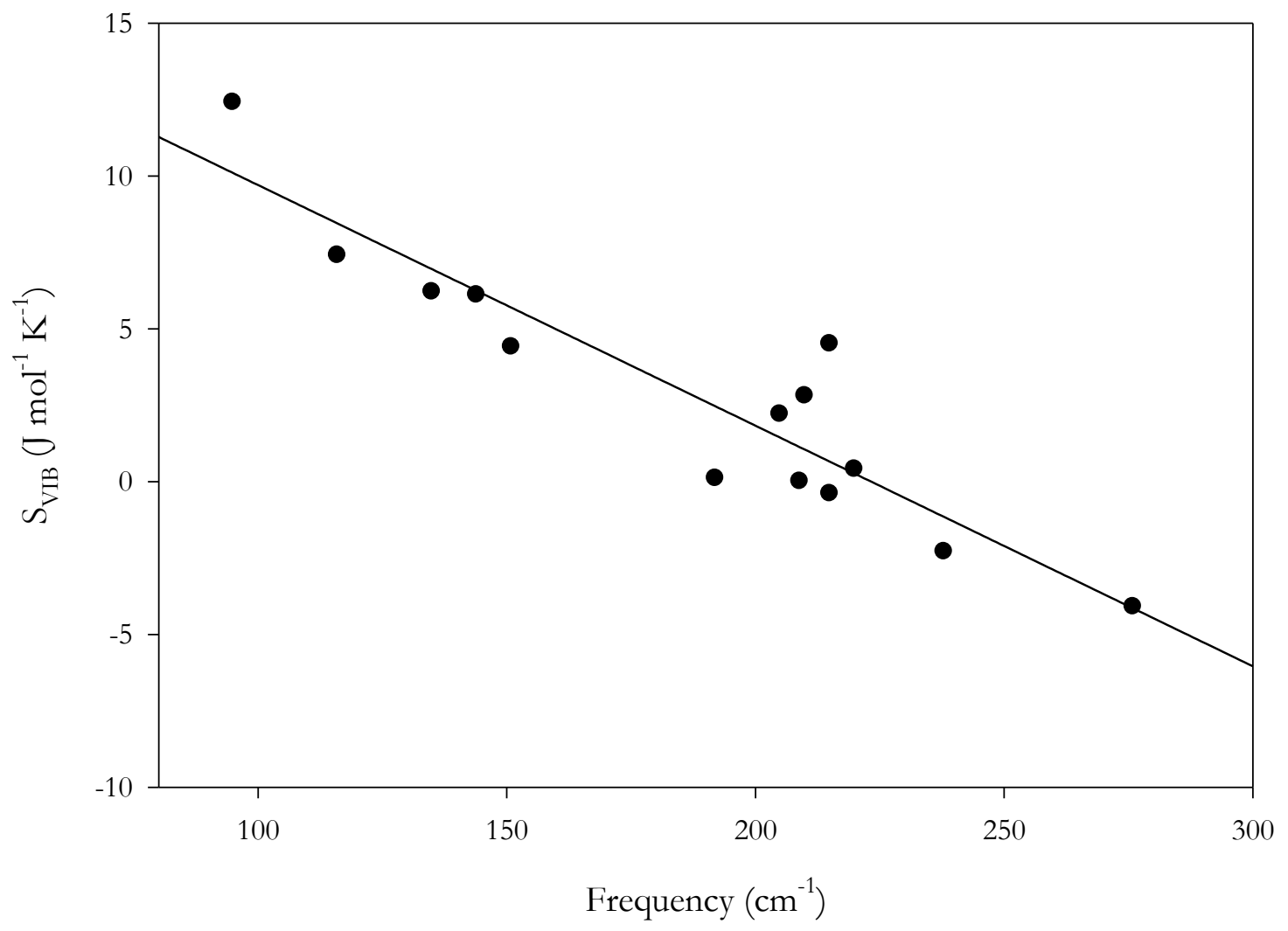

Fig. S.18. Correlation between the wavenumber associated with the vibration with the highest contribution from $\tau_{\mathrm{S} 1 \mathrm{C} 20}\left(v_{\tau \mathrm{S} 1 \mathrm{C} 20}\right.$, in $\left.\mathrm{cm}^{-1}\right)$ for most-stable conformers of MEAA to the vibrational contributions to entropy $\left(\Delta S_{\mathrm{vib}}\right.$, in $\mathrm{J} \mathrm{mol}^{-1} \mathrm{~K}^{-1}$ relatively to the most stable conformer) calculated at B3LYP/6-311++G(d,p) level. 